\title{
Transmission, Reflection and Absorption in Sonic and Phononic Crystals
}

A thesis submitted for the degree of Doctor of Philosophy by

\author{
Alejandro Cebrecos Ruiz \\ Instituto para la Gestión Integrada de Zonas Costeras \\ Universidad Politécnica de Valencia
}

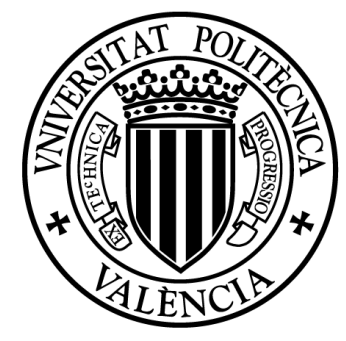

Valencia, October 6, 2015

SUPERVISORS

Víctor Sánchez Morcillo

Universidad Politécnica de Valencia, Spain

Rubén Picó Vila

Universidad Politécnica de Valencia, Spain

Dissertation JuRY

Vincent Tournat

Université du Maine (LAUM), France

Olivier Richoux

Université du Maine (LAUM), France

Francisco Camarena Femenía

Universidad Politécnica de Valencia, Spain 


The relevance of the people you love most is trully revealed when they are apart. Life goes on, memories remain.

To my brother, Toño. 
La relevancia de las personas que más quieres se manifiesta realmente cuando no están. La vida sigue, los recuerdos permanecen.

A mi hermano, Toño. 



\section{Aknowledgements}

It has been a long time since, after being involved for the first time in research during the realization of my Master Thesis, Víctor Sánchez gave me the oportunity to work with him to investigate subharmonic generation in resonant cavities. Soon after, he proposed me to start my $\mathrm{PhD}$ in the context of wave propagation in sonic crystals. I remember quite well my doubts and lack of confidence to start a PhD on this topic at that time. However, it was easy for Víctor to convince me, as science is a truly passion for him and he transmits it to everyone in his sorroundings. I am really grateful to him for sharing this years working together and totally convinced that I made the right choice. I also appreciate my other PhD supervisor, Rubén Picó, for his outstanding contribution and attitude, always open to discussion and helpful.

Fortunately, by the time I started my $\mathrm{PhD}$ studies, our research group experimented a quick growth and several researchers gradually started to work with us, enriching my work deeply. Vicent Romero worked in Gandía for two years, and still collaborate with us. He made a huge contribution to our research group and to this thesis. He helped me in many ways and situations, including numerical simulations, experimental techniques, and any doubt I might have. I also want to acknowledge Lluis García Raffi, who collaborated a lot with me too. He is always ready to share his knowledge in mathematics and physics with me and able to generate a huge amount of ideas and proposals. I also appreciate the contributions from other members of the group, such as Javier Redondo, and the people from ICREA in Terrasa. I appreciate the contributions from Kestutis Staliunas, whose astonishing knowledge of physics is always an inspiration to us, and Yu-Chieh Cheng, our collaborations working in periodic structures in optics and acoustics were of great interest for me and her extreme efficiency in every task she performs is amazing.

Chapter 5 of this thesis, and other works that are not included here, were developed in the University of Colorado at Boulder, in the USA, were I spent almost one year. I would like to acknowledge Mahmoud Hussein, from Dpt. of Aerospace Engineering Sciences, for giving me the oportunity to integrate myself in his group to work in the development of numerical techniques for elastic band structure calculation, and also for pushing hard to start and mantain a collaboration with our group in Gandía. It was an amazing experience were all the members of the group made me feel at home. Osama R. Bilal, Michael Frazier, Romik Khajehtourian, Dimitri Krattiger, Clémence Basquet and Hossein Honarvar, thank you all. 
Además de las personas con las que he trabajado en tareas directamente relacionadas con mi tesis, mi trabajo en Gandía ha sido muy placentero gracias a la gente del departamento con la que he compartido estos años. No quiero dejar pasar la oportunidad de agradecer el trabajo extremadamente profesional de Fernando Hernández, nuestro técnico del departamento y guardián de nuestros servidores de cálculo. Y por supuesto, todos los habitantes del Brain Zone, Noé, Patri, María, Vicent, Silvia, y Ester, así como las nuevas incorporaciones, Luis, Ahmed e Iván. Gracias a vosotros todo ha sido más divertido, sin olvidar lo que cada uno me habéis aportado tanto a nivel personal como profesional. Se os echará de menos!

Tras 10 años en Valencia y con mi familia a $800 \mathrm{~km}$ de aquí, han sido muchos los amigos con los que he compartido muchas vivencias en diferentes épocas y que se han convertido en mi familia valenciana, Alcoy, Pibe, Alvarico, Miguelón, Polo, Edouard, Patri, Dieguiño, Pascu, María, José Juan, Mario, Bosio, Pepo, Alex, José Corella, Fredo, Bart, Chino, Juanjo, Rasin. Han sido grandes años de los que me llevo grandes recuerdos. Aupa chavales!

Por último, quiero agradecer a mi familia por su permanente apoyo desde la distancia y por la motivación que me han transmitido mis padres, hermanas y mis sobrinetes. Especialmente a ti, Mamá, tú mejor que nadie sabes los rodeos que di al principio del camino que me ha llevado hasta aquí, y con tu esfuerzo y empuje inagotables eres la responsable de que haya llegado hasta el final, eres parte primordial de este trabajo. Te estaré eternamente agradecido y orgulloso de ti. 


\section{Abstract}

Phononic crystals are artificial materials formed by a periodic arrangement of inclusions embedded into a host medium, where each of them can be solid or fluid. By controlling the geometry and the impedance contrast of its constituent materials, one can control the dispersive properties of waves, giving rise to a huge variety of interesting and fundamental phenomena in the context of wave propagation. When a propagating wave encounters a medium with different physical properties it can be transmitted and reflected in lossless media, but also absorbed if dissipation is taken into account. These fundamental phenomena have been classically explained in the context of homogeneous media, but it has been a subject of increasing interest in the context of periodic structures in recent years as well. This thesis is devoted to the study of different effects found in sonic and phononic crystals associated with transmission, reflection and absorption of waves, as well as the development of a technique for the characterization of its dispersive properties, described by the band structure.

We start discussing the control of wave propagation in transmission in conservative systems. Specifically, our interest is to show how sonic crystals can modify the spatial dispersion of propagating waves leading to control the diffractive broadening of sound beams. Making use of the spatial dispersion curves extracted from the analysis of the band structure, we first predict zero and negative diffraction of waves at frequencies close to the band-edge, resulting in collimation and focusing of sound beams in and behind a 3D sonic crystal, and later demonstrate it through experimental measurements. The focusing efficiency of a 3D sonic crystal is limited due to the strong scattering inside the crystal, characteristic of the diffraction regime. To overcome this limitation we consider axisymmetric structures working in the long wavelength regime, as a gradient index lens. In this regime, the scattering is strongly reduced and, in an axisymmetric configuration, the symmetry matching with acoustic sources radiating sound beams increase its efficiency dramatically. Moreover, the homogenization theory can be used to model the structure as an effective medium with effective physical properties, allowing the study of the wave front profile in terms of refraction. We will show the model, design and characterization of an efficient focusing device based on these concepts.

Consider now a periodic structure in which one of the parameters of the lattice, such as the lattice constant or the filling fraction, gradually changes 
along the propagation direction. Chirped crystals represent this concept and are used here to demonstrate a novel mechanism of sound wave enhancement based on a phenomenon known as "soft" reflection. The enhancement is related to a progressive slowing down of the wave as it propagates along the material, which is associated with the group velocity of the local dispersion relation at the planes of the crystal. A model based on the coupled mode theory is proposed to predict and interpret this effect.

Two different phenomena are observed here when dealing with dissipation in periodic structures. On one hand, when considering the propagation of in-plane sound waves in a periodic array of absorbing layers, an anomalous decrease in the absorption, combined with a simultaneous increase of reflection and transmission at Bragg frequencies is observed, in contrast to the usual decrease of transmission, characteristic in conservative periodic systems at these frequencies. For a similar layered media, backed now by a rigid reflector, out-of-plane waves impinging the structure from a homogeneous medium will increase dramatically the interaction strength. In other words, the time delay of sound waves inside the periodic system will be considerably increased resulting in an enhanced absorption, for a broadband spectral range.

Finally, a new methodology for elastic band structure calculation is presented. Based on the finite-element in time-domain method, we consider a single unit cell applying Bloch boundary conditions depending on space and time, and compute the band structure by implementing a time-marching algorithm. A wide-band frequency signal excites the Bloch modes allowed to vibrate in the periodic structure and, by analyzing the time-history data, and spanning the Bloch wave vector along the Brillouin zone, we are able to detect these Bloch modes needed to build the dispersion relation of the system. The computational method is characterized in terms of accuracy, convergence and computation times. 


\section{Resumen}

Los cristales fonónicos son materiales artificiales formados por una disposición periódica de inclusiones embebidas en un medio, pudiendo ambos ser de carácter sólido o fluido. Controlando la geometría y el contraste de impedancias entre los materiales constituyentes se pueden controlar las propiedades dispersivas de las ondas, dando lugar a una enorme variedad de fenómenos fundamentales interesantes en el contexto de la propagación de ondas. Cuando una onda propagante se encuentra un medio con diferentes propiedades físicas puede ser transmitida y reflejada, en medios sin pérdidas, pero también absorbida, si la disipación es tenida en cuenta. Estos fenómenos fundamentales han sido explicados tradicionalmente en el contexto de medios homogéneos, pero en los últimos años también han sido objeto de un interés creciente en el contexto de estructuras periódicas. La presente tesis está dedicada al estudio de diferentes efectos presentes en cristales sónicos y fonónicos relacionados con la transmisión, reflexión y absorción de ondas, así como el desarrollo de una técnica para la caracterización de sus propiedades dispersivas, descritas por la estructura de bandas.

En primer lugar, se estudia el control de la propagación de ondas en transmisión en sistemas conservativos. Específicamente, nuestro interés se centra en mostrar cómo los cristales sónicos son capaces de modificar la dispersión espacial de las ondas propagantes, dando lugar al control del ensanchamiento de haces de sonido, característico de la difracción. Haciendo uso de las curvas de dispersión espacial extraídas del análisis de la estructura de bandas, se predice primero la difracción nula y negativa de ondas a frecuencias cercanas al borde de la banda, resultando en la colimación y focalización de haces acústicos en el interior y detrás de un cristal sónico $3 \mathrm{D}$, y posteriormente se demuestra mediante medidas experimentales. La eficiencia de focalización de un cristal sónico 3D está limitada debido a las múltiples reflexiones existentes en el interior del cristal, característico del régimen difractivo. Para superar esta limitación se consideran estructuras axisimétricas trabajando en el régimen de longitud de onda larga, como lentes de gradiente de índice. En este régimen, las reflexiones internas se reducen fuertemente y, en configuración axisimétrica, la adaptación de simetría con fuentes acústicas radiando haces de sonido incrementa la eficiencia drásticamente. Además, la teoría de homogenización puede ser empleada para modelar la estructura como un medio efectivo con propiedades físicas efectivas, permitiendo el estudio del frente 
de ondas en términos refractivos. Se mostrará el modelado, diseño y caracterización de un dispositivo de focalización eficiente basado en los conceptos anteriores.

Considérese ahora una estructura periódica en la que uno de los parámetros de la red, sea el paso de red o el factor de llenado, cambia gradualmente a lo largo de la dirección de propagación. Los cristales chirp representan este concepto y son empleados aquí para demostrar un mecanismo novedoso de incremento de la intensidad de la onda sonora basado en un fenómeno conocido como reflexión "suave". Este incremento está relacionado con una ralentización progresiva de la onda conforme se propaga a través del material, asociado con la velocidad de grupo de la relación de dispersión local en los planos del cristal. Un modelo basado en la teoría de modos acoplados es propuesto para predecir e interpretar este efecto.

Se observan dos fenómenos diferentes al considerar pérdidas en estructuras periódicas. Por un lado, si se considera la propagación de ondas sonoras en un array periódico de capas absorbentes, cuyo frente de ondas es paralelo a los planos del cristal, se produce una reducción anómala en la absorción combinada con un incremento simultáneo de la reflexión y transmisión a las frecuencias de Bragg, de forma contraria a la habitual reducción de la transmisión, característica de sistemas periódicos conservativos a estas frecuencias. En el caso de la misma estructura laminada en la que se cubre uno de sus lados mediante un reflector rígido, la incidencia de ondas sonoras desde un medio homogéneo, cuyo frente de ondas es perpendicular a los planos del cristal, produce un gran incremento de la fuerza de interacción. Dicho de otra forma, el tiempo de retardo de las ondas sonoras en el interior del sistema periódico aumenta considerablemente, resultando en un incremento de la absorción en un rango amplio de frecuencias.

Por último, se presenta una nueva metodología para el cálculo de estructuras de bandas en medios elásticos. Empleando el método de los elementos finitos en dominio temporal, se considera una celda unidad aplicando condiciones de contorno periódicas (Bloch) dependientes del espacio y el tiempo. Mediante una una señal de banda ancha se excitan los modos de Bloch permitidos por la estructura periódica y, mediante el análisis de las señales temporales registradas, así como el barrido del vector de onda de Bloch a lo largo de la zona de Brillouin, se detectan los modos de Bloch y obtener la relación de dispersion del sistema. El método computacional es caracterizado en términos de su precisión, convergencia y tiempo de computación. 


\section{Resum}

Els cristalls fonònics són materials artificials formats per una disposició d'inclusions en un medi, ambdós poden ser sòlids o fluids. Controlant la geometria i el contrast d'impedàncies dels seus materials constituents, és poden controlar les propietats dispersives de les ondes, permetent una gran varietat de fenòmens fonamentals interessants en el context de la propagació d'ones. Quan una ona propagant troba un medi amb pèrdues amb propietats físiques diferents es pot transmetre i reflectir, però també absorbida si la dissipació es té en compte. Aquests fenòmens fonamentals s'han explicat clàssicament en el context de medis homogenis, però també ha sigut un tema de creixent interés en el context d'estructures periòdiques en els últims anys. Aquesta tesi doctoral tracta de l'estudi de diferents efectes en cristalls fonònics i sònics lligats a la transmissió, reflexió i absorció d'ones, així com del desenvolupament d'una tècnica de caracterització de les propietats dispersives, descrites mitjançant la estructura de bandes.

En primer lloc, s'estudia el control de la propagació ondulatori en transmissió en sistemes conservatius. Més específicament, el nostre interés és mostrar com els cristalls sonors poden modificar la dispersió espacial d'ones propagants donant lloc al control de l'amplària per difracció dels feixos sonors. Mitjançant les corbes dispersió espacial obtingudes de l'anàlisi de l'estructura de bandes, es prediu, en primer lloc, la difracció d'ones zero i negativa a freqüències próximes al final de banda. El resultat és la collimació i focalització de feixos sonors dins i darrere de cristalls de so. Després es mostra amb mesures experimentals. L'eficiència de focalització d'un cristall de so 3D està limitada per la gran dispersió d'ones dins del cristall, que és característic del règim difractiu. Per a superar aquesta limitació, estructures axisimètriques que treballen en el règim de llargues longituds d'ona, i es comporten com a lents de gradient d'índex. En aquest règim, la dispersió es redueix enormement i, en una configuració axisimètrica, a causa de l'acoblament de la simetria $\mathrm{amb}$ les fonts acústiques que radien feixos sonors, l'eficiència de radiació s'incrementa significativament. D'altra banda, la teoria d'homogeneïtzació es pot utilitzar per a modelar, dissenyar i caracteritzar un dispositiu eficient de focalització basat en aquests conceptes.

Considerem ara una estructura periòdica en la qual un dels seus paràmetres de xarxa, com ara la constant de xarxa o el factor d'ompliment canvia 
gradualment al llarg de la direcció de propagació. Els cristalls chirped representen aquest concepte i s'utilitzen ací per a demostrar un mecanisme nou d'intensificació d'ones sonores basat en el fenòmen conegut com a reflexió "suau". La intensificació està relacionada amb la alentiment progressiva de l'ona conforme propaga al llarg del material, que està associada amb la velocitat de grup de la relació de dispersió local en els diferents plànols del cristall. Es proposa un model basat en la teoria de modes acoblats per a predir i interpretar este efecte.

Dos fenòmens diferents cal destacar quan es tracta d'estructures periòdiques amb dissipació. Per un costat, al considerar la propagació d'ones sonores en el plànol en un array periòdic de capes absorbents, s'observa una disminució anòmala de l'absorció i es combina amb un augment simultani de reflexió i transmissió en les freqüències de Bragg que contrasta amb la usual disminució de transmissió, característica dels sistemes conservatius a eixes freqüències. Per a un medi similar de capes, amb un reflector rígid darrere, les ones fora del pla incidint l'estructura des de un medi homogeni, augmentaran considerablement la interacció. En altres paraules, el retràs temporal de les ones sonores dins del sistema periòdic augmentarà significativament produint un augment d'absorció en un rang de banda ampla espectral.

Finalment, es presenta una nova metodologia per al càlcul de l'estructura de bandes en medis elàstics. Basat en el mètode d'elements finits en el domini de temps, considerem una cèllula unitat on s'apliquen les condicions de contorn de Bloch dependents de l'espai i del temps, i es calcula l'estructura de bandes implementant un algorisme de marxa. Un senyal amb gran amplada de banda excita els modes de Bloch que poden vibrar en l'estructura periòdica i, utilitzant les dades de temps enregistrades, i estenent els vectors d'ona de Bloch a la zona de Brillouin, és poden detectar els modes de Bloch necessaris per a construir la relació de dispersió del sistema. El mètode computacional es caracteritza mitjançant la precissió, convergencia i temps de càlcul. 




\section{Contents}

$\begin{array}{ll}\text { List of Publications } & \text { ii }\end{array}$

1 Introduction 1

1.1 Sonic and phononic crystals . . . . . . . . . . . 2

1.2 Transmission, reflection and absorption in periodic structures . 3

1.3 Object and motivation . . . . . . . . . . . . . . 7

1.4 Overview of Thesis . . . . . . . . . . . . . . . . . 12

1.4.1 Bibliographic notes . . . . . . . . . . . . 13

2 Control of wave propagation in transmission. Lensing effects 19

2.1 Focusing and collimation of acoustic waves by periodic structures 22

2.1.1 Positive, zero and negative diffraction: Isofrequency con-

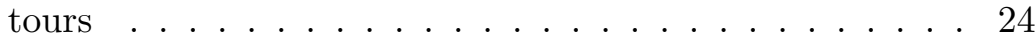

2.1.2 Paraxial approximation . . . . . . . . . . . 26

2.2 Wave focusing on axisymmetric structures . . . . . . . . 29

2.2 .1 Homogenization theory . . . . . . . . . . . . 31

2.2.2 Axisymmetric gradient index lens . . . . . . . . . 31

3 Reflection in chirped sonic crystals $\quad 39$

3.1 Coupled mode theory . . . . . . . . . . . . . . . . 41

3.1.1 CMT equations for linear propagation in fluids . . . . . . 41

3.1.2 Analytical solutions for linear and exponential profiles . . 43

3.2 Wave dispersion in chirped structures. Soft reflection . . . . . 48

3.2.1 Harmonic analysis. Spatial enhancement of the acoustic field . . . . . . . . . . . . . . . 49

3.2 .2 Time-domain analysis. . . . . . . . . . . . . 52

4 Absorption in periodic structures $\quad 59$

4.1 Enhanced transmission band in periodic structures with loss modulation . . . . . . . . . . . . . . 62 
4.1.1 Dissipative coupled mode theory . . . . . . . . . . . 62

4.1.2 Enhanced transmission in dissipative media . . . . . . . . 64

4.1 .3 Experimental study . . . . . . . . . . . . . . 65

4.2 Extraordinary absorption of sound in porous lamella-crystals . . 69

4.2 .1 Experimental verifications . . . . . . . . . . . 71

5 Band structure calculations in time-domain $\quad \mathbf{7 9}$

5.1 Unit-cell finite-element model . . . . . . . . . . . . . . . . 82

5.2 Unit-cell time-domain simulation . . . . . . . . . . . . 85

5.3 Numerical examples . . . . . . . . . . . . . . . . . . . . 88

5.4 Method performance . . . . . . . . . . . . . . . 91

$\begin{array}{lll}6 & \text { Concluding remarks } & 97\end{array}$

6.1 Conclusions . . . . . . . . . . . . . . . 97

6.1.1 Control of wave propagation in transmission . . . . . 98

6.1.2 Soft reflection in chirped sonic crystals . . . . . . . . . 99

6.1.3 Absorption in periodic layered media . . . . . . . . . . . 99

6.1.4 Band structure calculations in time-domain . . . . . . . . 100

6.2 Future work . . . . . . . . . . . . . . . . . 101

A Collection of publications $\quad 105$

A.1 Formation of collimated sound beams in 3D sonic crystals . . . 107

A.2 Wave focusing using symmetry matching in axisymmetric acoustic gradient index lenses . . . . . . . . . . . . . . 112

A.3 Enhancement of sound in chirped sonic crystals . . . . . . . 117

A.4 Enhancement of sound by soft reflections in exponentially chirped crystals . . . . . . . . . . . . . . . . . . 123

A.5 Enhanced transmission band in periodic media with loss modulation . . . . . . . . . . . . . . . . . 135

A.6 Extraordinary absorption of sound in porous lamella-crystals . . 141

B International conferences

C Awards 


\section{list of publications}

[1] A. Cebrecos, R. Picó, V. Romero-García, A. Yasser, L. Maigyte, R. Herrero, M. Botey, V. Sánchez-Morcillo, and K. Staliunas, "Enhanced transmission band in periodic media with loss modulation," Applied Physics Letters, vol. 105, no. 20, p. 204104, 2014.

[2] A. Cebrecos, R. Picó, V. Sánchez-Morcillo, K. Staliunas, V. Romero-García, and L. Garcia-Raffi, "Enhancement of sound by soft reflections in exponentially chirped crystals," AIP Advances, vol. 4, no. 12, p. 124402, 2014.

[3] A. Cebrecos, V. Romero-García, R. Picó, V. Sánchez-Morcillo, M. Botey, R. Herrero, Y. Cheng, and K. Staliunas, "Acoustically penetrable sonic crystals based on fluid-like scatterers," Journal of Physics D: Applied Physics, vol. 48, no. 2, p. 025501, 2015.

[4] A. Cebrecos, V. Romero-Garcia, R. Pico, I. Perez-Arjona, V. Espinosa, V. SanchezMorcillo, and K. Staliunas, "Formation of collimated sound beams by three-dimensional sonic crystals," Journal of Applied Physics, vol. 111, no. 10, p. 104910, 2012.

[5] J. Christensen, V. Romero-Garcia, R. Picó, A. Cebrecos, F. G. de Abajo, N. A. Mortensen, M. Willatzen, and V. Sánchez-Morcillo, "Extraordinary absorption of sound in porous lamella-crystals," Scientific reports, vol. 4, 2014.

[6] N. Jimenez, V. Romero-García, R. Picó, A. Cebrecos, V. J. Sánchez-Morcillo, L. GarciaRaffi, J. V. Sánchez-Pérez, and K. Staliunas, "Acoustic bessel-like beam formation by an axisymmetric grating," EPL (Europhysics Letters), vol. 106, no. 2, p. 24005, 2014.

[7] V. Romero-García, A. Cebrecos, R. Picó, V. J. Sánchez-Morcillo, L. Garcia-Raffi, and J. V. Sánchez-Pérez, "Wave focusing using symmetry matching in axisymmetric acoustic gradient index lenses," Applied Physics Letters, vol. 103, no. 26, p. 264106, 2013.

[8] V. Romero-García, R. Picó, A. Cebrecos, V. J. Sánchez-Morcillo, and K. Staliunas, "Enhancement of sound in chirped sonic crystals," Applied Physics Letters, vol. 102, no. 9, p. 091906, 2013.

[9] V. Romero-García, R. Picó, A. Cebrecos, K. Staliunas, and V. J. Sánchez-Morcillo, "Angular band gaps in sonic crystals: Evanescent waves and spatial complex dispersion relation," Journal of Vibration and Acoustics, vol. 135, no. 4, p. 041012, 2013. 



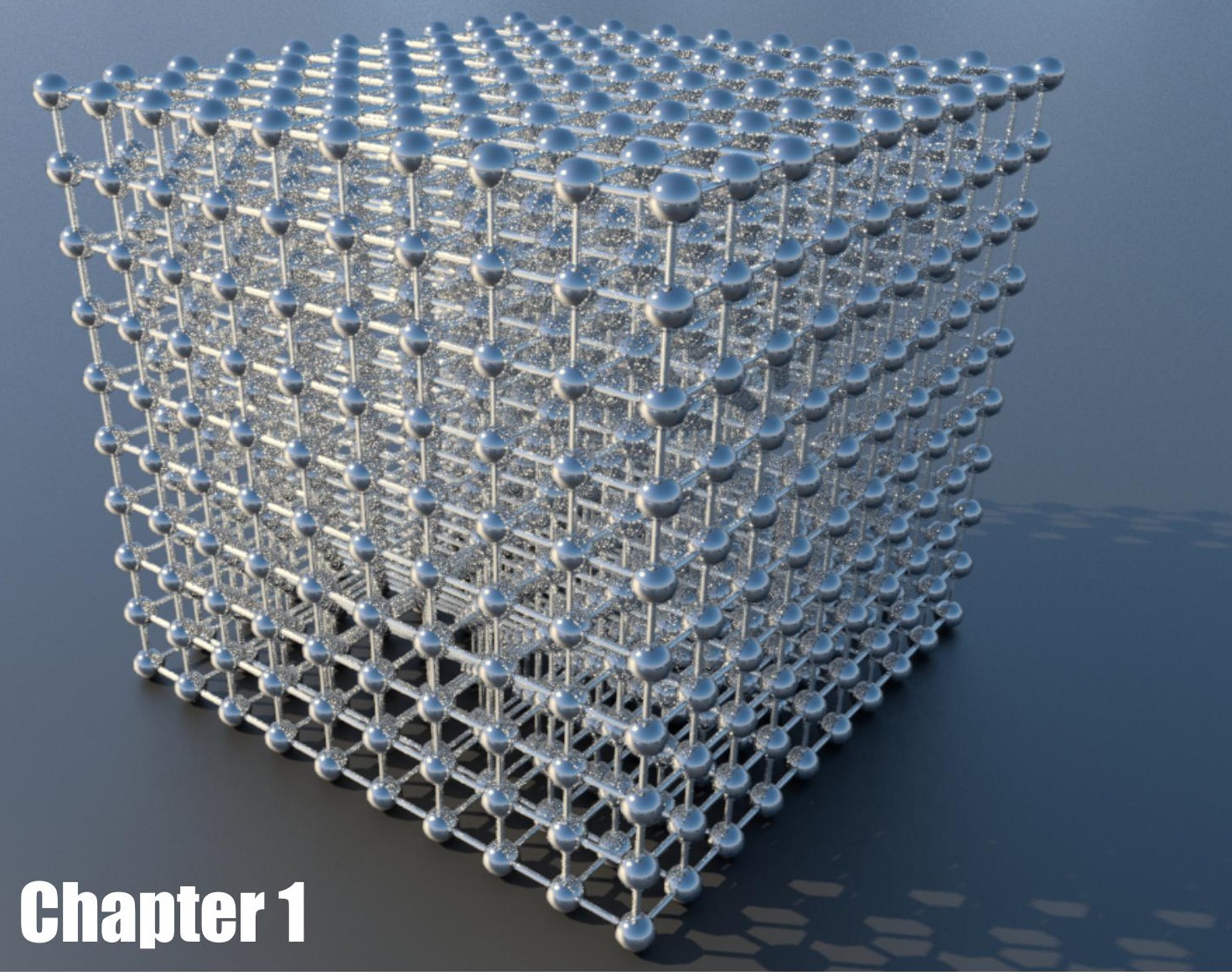

\section{Introduction}

This chapter is intended to offer a brief description of the physics of periodic media. A review of the state of the art is presented, mentioning historical origins and some of the most relevant works regarding transmission, reflection and absorption of waves in phononic crystals. Then, a description of the object and motivation of this thesis is given, commenting on several studies that triggered our interest to explore different phenomena presented throughout this work. Finally, a brief overview of the work and some bibliographic notes are given. 


\subsection{Sonic and phononic crystals}

In the late 80's, the pioneering studies of Yablonovitch [Yablonovitch, 1987] and John [John, 1987] pointed out the existence of photonic gaps as the electromagnetic counterpart of electron band gaps. Few years later, several theoretical works, inspired by the previous studies, ignite the interest in the study of acoustic and elastic and wave propagation in periodic materials presenting acoustic and elastic band structure calculations, showing the existence of acoustic and elastic band gaps, i.e., ranges of frequencies where vibrations, sound and phonons are forbidden [Sigalas and Economou, 1992, Sigalas and Economou, 1993, Kushwaha et al., 1993, Kushwaha, 1994].

Phononic crystals are artificial materials formed by a periodic arrangement of inclusions embedded into a host homogeneous material, featuring the propagation of longitudinal, transverse or mixed longitudinal and tranverse waves. A particular case of phononic crystal is the so-called sonic crystal, where the host material is a fluid and thus only longitudinal waves are allowed to propagate. By controlling the geometry and the impedance contrast of its constituent materials, one can control wave propagation and dispersion, giving rise to a huge variety of interesting and fundamental physical phenomena.

Band gaps ocurr due to Bragg resonances, i.e., destructive interference encountered at a particular range of frequencies. Most of the initial studies regarding phononic crystals were devoted to the existence of full band gaps and the required variations in the elastic constants and density to achieve this goal [Kushwaha et al., 1993, Kushwaha, 1994, Sigalas, 1997, Kushwaha et al., 1998], as well as experimental verifications of these phenomena [Martínez-Sala et al., 1995, Robertson and Rudy III, 1998, Sánchez-Pérez et al., 1998].

At the same time great efforts were made to develop methodology for band structure calculations. The plane wave expansion method (PWE) was applied in the initial works of Sigalas and Kushwaha [Sigalas and Economou, 1993, Kushwaha et al., 1993, Kushwaha, 1994]. In this method, by expanding the physical properties of the periodic media in Fourier series and applying Bloch's theory [Kittel and Holcomb, 1967], the wave equation is transformed into a set of linear homogeneous equations to form an eigenvalue problem that can be solved to obtain the band structure. Subsequently, other methods were explored with the same purpose. The multiple scattering method (MST) was used for the computation of the band structure [Kafesaki and 
Economou, 1999] and the determination of transmission and reflection coefficients for finite phononic crystals slabs [Liu et al., 2000]. Time-domain methods, such as the finite-difference in time domain (FDTD), were also explored for band structure calculations [Tanaka et al., 2000] and for the study of the transmission characteristics in two-dimensional finite phononic crystals [Garcia-Pablos et al., 2000].

Analysis of the band structure enables the study of a rich variety of fundamental phenomena and different wave propagation characteristics, with potential applications. In the next section we will briefly describe the state of the art by considering different effects in the context of tranmission, reflection and absorption in periodic media and finite-size phononic crystals, introducing some of the most relevant studies referenced in this thesis.

\subsection{Transmission, reflection and absorption in pe- riodic structures}

When a propagating wave encounters a medium with different physical properties it can be transmited and reflected in lossless media, but also absorbed if dissipation is taken into account. These fundamental phenomena have been classically explained in the context of homogeneous media, but it has been subject of increasing interest in the context of periodic structures in the last decades as well.

\section{Transmission phenomena}

One of the hot topics in the last decades regarding the control of wave propagation in transmission in sonic and phononic crystals has been the focusing of acoustic waves. There are several ways to achieve this goal, but arguably negative refraction is the most explored method. It is predicted analyzing the equifrequency contours of the band structures, as their gradient vectors in the reciprocal space give the group velocity of waves inside the crystal, and thus, the direction of propagation. It was first demonstrated theoretically and experimentally for acoustic waves more than a decade ago [Zhang and Liu, 2004, Yang et al., 2004]. Two different mechanisms have been reported for wave focusing using negative refraction; on one hand, it can be achieved due to Bragg scattering near the Brillouin zone in the first band without showing 
a negative refractive index, as reported by Zhang and Liu [Zhang and Liu, 2004]. On the other hand, it can also be found in the second band due to a negative effective index [Yang et al., 2004]. Several works explored the characteristics of this effect later [Ke et al., 2005, Feng et al., 2005, Qiu et al., 2005,Li et al., 2006, Sukhovich et al., 2008, Bucay et al., 2009], and it was used for the design of lenses based in phononic crystals slabs, where incident waves from point sources where focused at the other side of the slab, creating an image. Moreover, this effect was soon oriented to the design of perfect lenses, where the coupling of the incident field with evanescent modes inside the crystals may lead to overcome the difraction limit, like reported initially in photonics [Luo et al., 2003] and later in phononics [Ke et al., 2007a, Sukhovich et al., 2009]. Other methods have demonstrated that phononic crystals can also be used to generate focusing on the far field [Qiu et al., 2005] or to obtain highly directional beams in different manners, making use of the band edge states [Ke et al., 2007a] or by resonant cavities at frequencies belonging to the band gap [Hsu et al., 2008, Wu et al., 2006, Ke et al., 2006].

Another interesting effect in transmission is the non-diffractive propagation of waves in periodic systems, named self-collimation. This effect is based on the existence of flat segments of spatial dispersion curves (the curves of constant frequency in $\mathrm{k}$-space) at particular frequencies depending on the geometry of the crystal. It was first demonstrated experimentally for electromagnetic waves in the optical and microwave regimes, and later reported for acoustic waves [Pérez-Arjona et al., 2007, Espinosa et al., 2007].

The aforementioned effects occur for frequencies at the band gap, or at frequencies close to the band edges, below or above the band gap, where the wavelength is of the same order as the separation between inclusions, i.e., the lattice constant. A different regime, named the long-wavelength regime, is found for frequencies well below the Bragg resonances, where the wavelength is large compared to the lattice constant. In this regime, periodic structures can be considered homogeneous materials having effective properties. Cervera et al presented a simple model that allowed to use a sonic crystal as a refractive focusing device for sound focusing [Cervera et al., 2001]. The first exact analytical theory of homogenization of periodic elastic structures was based in the PWE method, obtaining compact formulas for the effective velocity [Krokhin et al., 2003]. Few years later, not only the effective velocity but also the effective mass density were derived using the multiple scattering theory [Mei et al., 2006, Torrent and Sánchez-Dehesa, 2006, Torrent et al., 2006]. 
Subsequent works presented different designs of gradient index lenses based on phononic crystals in the long-wavelength regime, changing the elastic properties of cylinders along the transverse direction [Lin et al., 2009], or using unit cells based on cross-shape scatterers [Zigoneanu et al., 2011] and coiling up space [Li et al., 2012], achieving a high transmission efficiency usign small devices compared to the wavelength.

\section{Reflection phenomena}

So far we have described several works considering different transmission effects in phononic crystals. It is very well-known that propagating waves impinging a periodic structure are efficiently back reflected at frequencies belonging to the band gap (i.e., the Bragg frequencies). In this regime, most studies have been traditionally focused in studying the absence of transmission [Martínez-Sala et al., 1995,de Espinosa et al., 1998,Sánchez-Pérez et al., 1998], defect modes [Khelif et al., 2003, Romero-García et al., 2010] and related phenomena. However, few of them explored what are the characteristics of wave reflection, in other words, how waves are reflected and what effects can be found.

The reflection properties in finite-size sonic crystals were studied more than a decade ago using the so-called standing-wave ratio technique, which constitutes a measure of the partial standing wave formed in front of a sonic crystal when a sound wave impinges on it, at frequencies belonging to the band gap [Sanchis et al., 2001]. In this context, another study calculated the reflection coefficient to measure the sound velocity inside a refractive device in the long wavelength regime, as an example of a Fabry-Pérot interferometer [Cervera et al., 2001].

Reflection of waves at the interface defined by the host and the periodic media has been the subject of several studies. Specifically, the diffraction of bulk waves on the exterior surface of a two-dimensional phononic crystal was studied, showing the existence of orders of diffraction, behaving as a diffraction grating [Moiseyenko et al., 2012, Moiseyenko et al., 2013]. In the case of electromagnetic waves, recent works demonstrated the near-field focusing of narrow beams in reflection from flat subwavelength diffraction gratings, reporting numerical [Cheng et al., 2014b], and experimental [Cheng et al., 2014c] evidences. Following the concept of focusing in reflection, Cheng et al considered flat chirped structures as an efficient device to focus light beams 
in reflection [Cheng et al., 2013, Cheng et al., 2014a, Cheng et al., 2015].

Chirped crystals, also called graded or adiabatic tappered, have been used in optics for different purposes, such as light bending at frequencies near the band gap [Cassan et al., 2011]. An interesting phenomenon shown in photonic chirped crystals is the smooth deflection of a light beam from the straight trajectory as it propagates through the crystal, the so-called mirage effect [Centeno et al., 2006]. Another interesting effect reported recently is the so-called rainbow trapping effect, the dependence of the turning point position on the color of radiation. It has been predicted for one-dimensionally modulated chirped photonic structures [Shen et al., 2011] and tapered optical and plasmonic waveguides [Shen et al., 2011,Smolyaninova et al., 2010]. These type of crystals were introduced in acoustics some time ago to open full band gaps [Kushwaha et al., 1998, Psarobas and Sigalas, 2002] or as an acoustic bending waveguide [Wu and Chen, 2011]. However, we are not aware of works in the field of acoustics exploring some of the aforementioned effects in wave propagation using these structures, such as the mirage effect or the rainbow trapping.

\section{Dissipation in phononic crystals}

Although many effects found in periodic media can be observed in conservative systems, waves cannot be absorbed unless dissipation is considered. Moreover, especially in real experiments, often one or more of the constituent materials present some non-negligible losses in the frequency range of interest, which might introduce some discrepancies with theoretical and numerical predictions if a conservative system is considered.

The effect of viscoelastic losses in phononic crystals was discussed more than a decade ago using a finite crystal with a unit cell of close-packed rubber spheres in air, analyzing its transmittance and absorbance under a multiple scattering approach [Psarobas, 2001]. More recent studies discussed the modification of the dispersion relation resulting in phononic crystals composed of lossless inclusions in a solid lossy matrix [Laude et al., 2013], or the influence of the material and size inclusions in a lossy solid matrix on wave attenuation [Hwan Oh et al., 2013]. Damping of elastic waves in solid-solid phononic crystals has also been discussed, as in Ref. [Andreassen and Jensen, 2013] or by Hussein, who proposed a theory for Bloch wave propagation in viscous damped elastic media that allow to compute the band structures using a finite 
element framework [Hussein, 2009].

Another posibility to introduce losses in a periodic system is to consider porous materials, where viscothermal losses are dominant. Wave propagation in such materials has been investigated to calculate the band structure incorporating absorption in a periodic layered acoustic media [Lee et al., 2010], or to describe wave propagation in the long-wavelength regime in an acoustic absorbing media with rigid periodic inclusions [Tournat et al., 2004].

In the audible regime most of the studies have been, and still are, trying to achieve maximum absorption for a broadband frequency range and for incident radiation at any angle. Sonic crystals made of rigid cylinders with a porous absorbent covering [Umnova et al., 2006], or a periodic array of scatterers made of a combination of rigid, absorbent and resonant cavities [Romero-García et al., 2011] have been proposed as efficient acoustic devices to block sound. Other recent studies considered elastic membranes [Mei et al., 2012] or microperforated shells [García-Chocano et al., 2012] to increase the absorption and at the same time, reduce the reflection.

\subsection{Object and motivation}

The main object of this work is the study of different phenomena associated with transmission, reflection and absorption of acoustic waves in phononic crystals. In particular, we concentrate our efforts in three different topics; (i) wave focusing and collimation in transmission, (ii) field enhancement using the concept of soft reflection in chirped structures, and (iii) the control of losses in layered dissipative systems. To do that, we use 1D, 2D and 3D finite structures of various types, materials and geometries. Nonetheless, all the systems considered here posses a common property, a fluid is selected as the host medium. Hence, we deal with sonic crystals, where only longitudinal waves propagate in the host medium. Depending on the case, the fluid considered is air or water and the corresponding range of frequencies lay on the audible and ultrasonic regimes, respectively. Moreover, all the structures considered are analyzed both theoretically and experimentally.

A secondary goal of this work is the development of a computational methodology for elastic band structure calculation in the framework of the finite element in time-domain method, using $1 \mathrm{D}$ and 2D solid-solid phononic crystals. In this case, we consider the propagation of longitudinal (1D) and 
coupled in-plane longitudinal and shear vertical wave propagation modes (2D). This work was developed under the supervision of Prof. Mahmoud Hussein at the Department of Aerospace Engineering Sciences at the University of Colorado at Boulder as part of the program VLC/Campus para promover la internacionalización de la Formación Doctoral mediante la realización de Estancias en Centros Extranjeros de Prestigio.

Having these goals in mind, we describe now several works that motivated us to achieve them.

\section{Wave propagation control in transmission}

Most of the works mentioned in the previous section regarding focalization considered point sources to generate the acoustic field, in contrast to sound beams with a radiation pattern similar to real acoustic sources with radial symmetry [Huang and Breazeale, 2006]. Several studies developed in our research group considered the non-diffractive propagation and focalization of sound beams in two-dimensional sonic crystals [Pérez-Arjona et al., 2007, Espinosa et al., 2007]. In these works, analytical expressions for the prediction of nondiffractive propagating beams are presented, considering the field expansion of the homogeneous and low order modes and the analysis of their spatial dispersion curves. Focalization of sound beams behind 2D sonic crystals was also considered in [Sánchez-Morcillo et al., 2009]. Similar analytical methods (i.e., field expansions) were used in this work to predict and identify different focusing regimes, as well as focusing distances and beam widths behind the crystal, demonstrating the validity of these expressions through numerical simulations and experimental results.

Motivated by the previous theoretical and experimental studies in twodimensional systems, we extend here these works in order to explore the focusing and collimation characteristics in three-dimensional sonic crystals. For that purpose, we study the spatial dispersion curves and the focusing characteristics, such as the focusing distance or the beam width, using the explicit formulas derived and presented in those studies. An interesting effect that arises from the results obtained previously is the possibility to use sonic crystals to modify the focusing distance by slightly varying the frequency of the incident acoustic beam. On the other hand, as stated previously, most of the existing acoustic wave sources posses radial symmetry. Hence, it seems relevant to consider the symmetry matching between the source and the focusing 
device in the design of acoustic lenses. With this regard, we are interested in improving the efficiency of acoustic lenses exploring the modelling and design of axisymmetric structures as focusing devices.

\section{Wave enhancement in chirped crystals}

Chirped crystals have been used in acoustics mainly to open full band gaps [Kushwaha et al., 1998,Psarobas and Sigalas, 2002] or as an acoustic bending waveguide [Wu and Chen, 2011], but few attention has been paid to benefit from the possibilities of wave control arising from the gradual variation of the properties of the crystal, characteristic of chirped structures. In particular, the study of phenomena related to the reflection of acoustic waves using these structures. In optics, chirped crystals have been succesfully employed for different purposes, and the local variation of the group velocity has been used to demonstrate the mirage effect [Centeno et al., 2006], or the rainbow trapping effect [Shen et al., 2011], among them.

Motivated by these works in optics, and considering the similarities between electromagnetic and acoustic waves, we explore this phenomenos in acoustics. Moreover, we are willing to study the influence of the local variation of the wave group velocity in its propagation along a one-dimensional chirped crystal. We demonstrate here that, using chirped structures in which the lattice constant is reduced along the propagation direction, and working at frequencies close to the upper band edge, it is possible to gradually reduce the sound velocity of the wave until it reachess a plane having a band gap at a given frequency, leading to a soft reflection and subsequent enhancement of the acoustic field at a "brilliant" plane. Moreover, the position of this "brilliant" plane inside a 1D chirped structure will depend on the frequency of the incident radiation, an unambiguous indication of the rainbow trapping effect. A coupled mode theory, widely used in optics, is considered here to its application in acoustics to predict the existence of the aforementioned effects in acoustic chirped crystals.

\section{Dissipative periodic structures}

The control of losses using periodic systems, generally speaking, is a now topic of great interest, not only for acoustic or elastic waves, but also for 
electromagnetic waves. In optics, many efforts are devoted to minimize losses with different purposes. Some studies reported absorption reduction in a multilayered magneto-photonic crystals [Figotin and Vitebskiy, 2008, Figotin and Vitebskiy, 2010], while others achieved enhanced transmission through a stack of dielectric layers having contrast only in attenuation [Erhokhin et al., 2008, Kumar et al., 2012], and extended to two-dimensional (2D) modulation of losses, showing nontrivial light beam propagation effects, analogous to flat photonic crystal lensing reported in conservative systems [Staliunas et al., 2009, Kumar et al., 2013].

These studies in optics motivated our interest to investigate the control of losses in acoustics from a fundamental point of view. To do that, we investigate in-plane wave propagation in a layered system formed by a periodic array of porous absorbing layers embedded in air. Our interest is to explore how the periodicity of the absorptive system modifies the global absorption as well as the reflection and transmission properties. We will show here how a simultaneous increase of transmission and reflection, combined with a decrease in the global absorption, ocurrs around the Bragg frequency. This anomalous behavior is different from the classical decrease in transmission characteristic in conservative systems at this range of frequencies. Now, as in the case of reflection in chirped structures, a coupled mode theory is used, which is extended to include losses that allow us to predict the effect, which will be demonstrated experimentally as well.

In the audible regime, from a technological point of view, there is a huge interest in the development of systems featuring complete absorption of sound, meaning a broadband absorption and for multiple directions of the incident field. Moreover, due to the large wavelength of sound waves at low frequency, the absorption of sound is only achieved by means of very thick absorbers. Different systems based on periodic structures constitute an option to develop efficient absorbers [Umnova et al., 2006]. However, the behavior of periodic systems is highly dependent on frequency, specially in systems incorporating rigid scatterers. Thus, the development of efficient absorbers based in sonic crystals often incorporates additional effects to compensate the lack of a broadband behavior [Romero-García et al., 2011, García-Chocano et al., 2012, Mei et al., 2012]. In this context, we analyze experimentally a periodic system similar to the one mentioned previously for in-plane wave propagation, formed by a periodic distribution of porous layers. However, the periodic layered media is designed for out-of-plane waves impinging the structure from a homogeneous medium and it is backed by a perfect reflector. This configura- 
tion allows to increase dramatically the time delay of sound waves inside the periodic system, resulting resulting in an enhanced absorption, for a broadband spectral range in different angles of the incident radiation.

\section{Characterization of dispersive properties in phononic crystals}

Several methods for the computation of the band structure in phononic crystals are already available, all of them with their pros and cons. Most of the existent methods are formulated in the context of time-harmonic dependence, i.e., assuming steady-state solutions and incorporating Bloch theory in most cases, allowing to consider a single unit cell in the calculations. PWE is one of the most popular, being suitable for solid-solid or fluid-fluid unit cells [Kushwaha et al., 1993, Sigalas and Economou, 1993]. Multiple scattering methods (MST) are also a good option, and they easily deal with fluid-solid unit cells [Kafesaki and Economou, 1999,Kafesaki et al., 2000,Liu et al., 2000]. Other methods compute the band structures in time-domain, postprocessing the time-history data, such as FDTD.

Our motivation here is the development of a methodology based in the finite-element in time-domain. The idea is, on one hand, to overcome the existent limitation in MST or FDTD to deal with irregularly shaped scatterers, something achieved easily by employing a finite element discretization of the unit cell and at the same do that in a framework capable to deal with solid-fluid unit cells. On the other hand, the motivation to implement a timedomain technique is to employ this method to a problem where the unit cell is composed of a solid and a fluid channel where the fluid is time-dependent. In this thesis, however, we will only consider a solid-solid unit cell in order to validate the method. The fluid channel problem is a work in progress at the time this thesis is written and the subsequent publication will be submitted to a journal soon. Nontheless, this work has been presented already Phononics 2015: 3rd International Conference on Phononic Crystals/Metamaterials, Phonon Transport and Phonon Coupling under the title: An elastic medium with a time-changing band structure. 


\subsection{Overview of Thesis}

A brief description of the organization of the contents of this thesis is described here. This document contains 6 Chapters and 1 Appendix. This work encompass the study of transmission, reflection and absorption effects found in sonic and phononic crystals and the computation of their dispersive properties is also considered. The thesis is written in the form of a collection of articles which are included in Appendix A. The main concepts, methods and results of these publications are described extensively along this document.

Chapter 2 is devoted to the study of transmission effects in sonic crystals. The focusing and collimation in regular periodic and axisymmetric structures is studied in two different regimes, the long wavelength and the diffractive regimes. Using theoretical tools such as the eigenfrequency analysis using the finite element method, or the homogenization theory, we can predict the focusing behavior of finite-size structures and characterize it using numerical simulations and experimental measurements.

The study of wave phenomena in chirped sonic crystals is considered in Chapter 3. In order to model the reflection of waves in this peculiar structures, in which one of the parameters of the structure varies along the propagation direction, we consider and describe in detail a coupled mode theory, adapted here to model the propagation of waves in acoustic layered media. This theory is widely used in optics in the context of photonic crystals to model the propagation of coupled forward and backward waves. Using this theory, a wave enhancement found in chirped sonic crystals related to a progressive slowing down of waves is predicted and demonstrated using analitical, numerical and experimental methods.

Up to this point, all the systems considered are conservative. Now we turn our attention to dissipative systems, consideration the effect of absorption in sonic crystals. In Chapter 4 two multilayered systems of periodic absorbers are considered for different purposes. On one hand, the enhancement of transmission of in-plane waves around the Bragg frequencies is analyzed. For that purpose, we consider an extension of the couple mode theory used in Chapter 3 incorporating losses, and discuss the differences found in the main parameters of the theory with respect to conservative systems. We will bring some insights to show how this enhancement of transmission is related to a decrease in the absorption in the lossy layers. On the other hand, we will discuss how a slight variation in the configuration of a similar layered system can be used 
for a different purpose, the enhancement of the absorption. We demonstrate through experimental measurements a dramatic increase in the absorption when out-of plane-waves strike a multilayered medium of porous absorbers backed by a rigid reflector.

We will turn our interest later in the characterization of the dispersive properties in phononic crystals. A new methodology for band structure calculation is proposed in Chapter 5. The proposed method is based in timedomain simulations and implemented in the framework of the finite element method. We will discuss the characteristics of the method by comparing its performance, in terms of accuracy, convergence and computational time, with other standard and already validated methods for band structure calculations.

Ultimately, Chapter 6 summarizes the work describing the main conclusions and offering some perspectives for future research.

\subsubsection{Bibliographic notes}

The references of this work are included at the end of each chapter in order to facilitate the search of cited references by the reader.

\section{References}

Andreassen, E. and Jensen, J. S. (2013). Analysis of phononic bandgap structures with dissipation. Journal of Vibration and Acoustics, 135(4):041015.

Bucay, J., Roussel, E., Vasseur, J., Deymier, P. A., Hladky-Hennion, A.-C., Pennec, Y., Muralidharan, K., Djafari-Rouhani, B., and B, D. (2009). Positive, negative, zero refraction and beam splitting in a solid/air phononic crystal: Theoretical and experimental study. Physical Review B, 79:214305-214305-7.

Cassan, E., Do, K.-V., Caer, C., Marris-Morini, D., and Vivien, L. (2011). Shortwavelength light propagation in graded photonic crystals. Lightwave Technology, Journal of, 29(13):1937-1943.

Centeno, E., Cassagne, D., and Albert, J.-P. (2006). Mirage and superbending effect in two-dimensional graded photonic crystals. Phys. Rev. B, 73:235119.

Cervera, F., Sanchis, L., Sanchez-Perez, J., Martinez-Sala, R., Rubio, C., Meseguer, F., López, C., Caballero, D., and Sánchez-Dehesa, J. (2001). Refractive acoustic devices for airborne sound. Phys. Rev. Lett., 88:023902.

Cheng, Y., Peckus, M., Kicas, S., Trull, J., Cojocaru, C., Vilaseca, R., Drazdys, R., and Staliunas, K. (2013). Beam focusing in reflection from flat chirped mirrors. Physical Review $A, 87(4): 045802$. 
Cheng, Y.-C., Kicas, S., and Staliunas, K. (2015). Flat focusing in reflection from a chirped dielectric mirror with a defect layer. Journal of Nanophotonics, 9(1):093084-093084.

Cheng, Y. C., Kicas, S., Trull, J., Peckus, M., Cojocaru, C., Vilaseca, R., Drazdys, R., and Staliunas, K. (2014a). Flat focusing mirror. Scientific reports, 4.

Cheng, Y. C., Redondo, J., and Staliunas, K. (2014b). Beam focusing in reflections from flat subwavelength diffraction gratings. Physical Review A, 89(3):033814.

Cheng, Y. C., Zeng, H., Trull, J., Cojocaru, C., Malinauskas, M., Jukna, T., Wiersma, D., and Staliunas, K. (2014c). Beam focalization in reflection from flat dielectric subwavelength gratings. Optics letters, 39(20):6086-6089.

de Espinosa, F. M., Jimenez, E., and Torres, M. (1998). Ultrasonic band gap in a periodic two-dimensional composite. Physical Review Letters, 80(6):1208.

Erhokhin, S. G., Lisyansky, A. A., Merzlikin, A. M., Vinogradov, A. P., and Granovsky, A. B. (2008). Photonic crystals built on contrast in attenuation. Phys. Rev. B, 77:233102.

Espinosa, V., Sánchez-Morcillo, V. J., Staliunas, K., Pérez-Arjona, I., and Redondo, J. (2007). Subdiffractive propagation of ultrasound in sonic crystals. Physical Review B, $76(14): 140302$.

Feng, L., Liu, X.-P., Chen, Y.-B., Huang, Z.-P., Mao, Y.-W., Chen, Y.-F., Zi, J., and Zhu, Y.-Y. (2005). Negative refraction of acoustic waves in two-dimensional sonic crystals. Physical Review B, 72(3):033108.

Figotin, A. and Vitebskiy, I. (2008). Absorption suppression in photonic crystals. Phys. Rev. B, 77:104421.

Figotin, A. and Vitebskiy, I. (2010). Magnetic faraday rotation in lossy photonic structures. Waves in Random and Complex Media, 20(2):298-318.

García-Chocano, V. M., Cabrera, S., and Sánchez-Dehesa, J. (2012). Broadband sound absorption by lattices of microperforated cylindrical shells. Applied Physics Letters, 101(18):184101.

Garcia-Pablos, D., Sigalas, M., De Espinosa, F. M., Torres, M., Kafesaki, M., and Garcia, N. (2000). Theory and experiments on elastic band gaps. Physical Review Letters, 84(19):4349.

Hsu, F.-C., Wu, T.-T., Hsu, J.-C., and Sun, J.-H. (2008). Directional enhanced acoustic radiation caused by a point cavity in a finite-size two-dimensional phononic crystal. Applied Physics Letters, 93(20):201904.

Huang, D. and Breazeale, M. A. (2006). An ultrasonic gaussian transducer and its diffraction field: theory and practice. Ultrasonics, Ferroelectrics, and Frequency Control, IEEE Transactions on, 53(5):1018-1027.

Hussein, M. I. (2009). Theory of damped bloch waves in elastic media. Phys. Rev. B, $80: 212301$.

Hwan Oh, J., Jae Kim, Y., and Young Kim, Y. (2013). Wave attenuation and dissipation mechanisms in viscoelastic phononic crystals. Journal of Applied Physics, 113(10).

John, S. (1987). Strong localization of photons in certain disordered dielecric superlattices. Physical Review Letters, 58(23):2486-2489. 
Kafesaki, M. and Economou, E. N. (1999). Multiple-scattering theory for three-dimensional periodic acoustic composites. Physical review B, 60(17):11993.

Kafesaki, M., Penciu, R., and Economou, E. (2000). Air bubbles in water: a strongly multiple scattering medium for acoustic waves. Physical review letters, 84(26):6050.

Ke, M., Liu, Z., Cheng, Z., Li, J., Peng, P., and Shi, J. (2007). Flat superlens by using negative refraction in two-dimensional phononic crystals. Solid State Communications, 142(3):177-180.

Ke, M., Liu, Z., Pang, P., Wang, W., Cheng, Z., Shi, J., Zhao, X., and Wen, W. (2006). Highly directional acoustic wave radiation based on asymmetrical two-dimensional phononic crystal resonant cavity. Applied physics letters, 88(26):263505.

Ke, M., Liu, Z., Qiu, C., Wang, W., Shi, J., Wen, W., and Sheng, P. (2005). Negative-refraction imaging with two-dimensional phononic crystals. Physical Review B, 72(6):064306.

Khelif, A., Choujaa, A., Djafari-Rouhani, B., Wilm, M., Ballandras, S., and Laude, V. (2003). Trapping and guiding of acoustic waves by defect modes in a full-band-gap ultrasonic crystal. Phys. Rev. B, 68:214301.

Kittel, C. and Holcomb, D. F. (1967). Introduction to solid state physics. American Journal of Physics, 35(6):547-548.

Krokhin, A., Arriaga, J., and Gumen, L. (2003). Speed of sound in periodic elastic composites. Physical review letters, 91(26):264302.

Kumar, N., Botey, M., Herrero, R., Loiko, Y., and Staliunas, K. (2012). High-directional wave propagation in periodic loss modulated materials. Photonics and Nanostructures Fundamentals and Applications, 10(4):644 - 650. TaCoNa-Photonics 2011.

Kumar, N., Herrero, R., Botey, M., and Staliunas, K. (2013). Flat lensing by periodic loss-modulated materials. J. Opt. Soc. Am. B, 30(10):2684-2688.

Kushwaha, M. (1993). Acoustic band structure of periodic elastic composites. Physical Review Letters, 71(13):2022-2025.

Kushwaha, M. (1994). Theory of acoustic band structure of periodic elastic composites. Physical Review B, 49(4):2313-2322.

Kushwaha, M., Djafari-Rouhani, B., Dobrzynski, L., and Vasseur, J. (1998). Sonic stopbands for cubic arrays of rigid inclusions in air. The European Physical Journal B Condensed Matter and Complex Systems, 3(2):155-161.

Laude, V., Escalante, J. M., and Martínez, A. (2013). Effect of loss on the dispersion relation of photonic and phononic crystals. Phys. Rev. B, 88:224302.

Lee, C.-Y., Leamy, M. J., and Nadler, J. H. (2010). Frequency band structure and absorption predictions for multi-periodic acoustic composites. Journal of Sound and Vibration, 329(10):1809 - 1822 .

Li, J., Liu, Z., and Qiu, C. (2006). Negative refraction imaging of acoustic waves by a two-dimensional three-component phononic crystal. Physical Review B, 73(5):054302.

Li, Y., Liang, B., Tao, X., Zhu, X.-f., Zou, X.-y., and Cheng, J.-c. (2012). Acoustic focusing by coiling up space. Applied Physics Letters, 101(23):233508. 
Lin, S.-C. S., Huang, T. J., Sun, J.-H., and Wu, T.-T. (2009). Gradient-index phononic crystals. Physical Review B, 79(9):094302.

Liu, Z., Chan, C., Sheng, P., Goertzen, A., and Page, J. (2000). Elastic wave scattering by periodic structures of spherical objects: Theory and experiment. Physical Review B, 62(4):2446.

Luo, C., Johnson, S. G., Joannopoulos, J. D., and Pendry, J. B. (2003). Subwavelength imaging in photonic crystals. Physical Review B, 68:045115-045115-14.

Martínez-Sala, R., Sancho, J., Sánchez, J. V., Gómez, V., Llinares, J., and Meseguer, F. (1995). Sound attenuation by sculpture. Nature, 378:241.

Mei, J., Liu, Z., Wen, W., and Sheng, P. (2006). Effective mass density of fluid-solid composites. Physical review letters, 96(2):024301.

Mei, J., Ma, G., Yang, M., Yang, Z., Wen, W., and Sheng, P. (2012). Dark acoustic metamaterials as super absorbers for low-frequency sound. Nature Communications, 3:756.

Moiseyenko, R. P., Herbison, S., Declercq, N. F., and Laude, V. (2012). Phononic crystal diffraction gratings. Journal of Applied Physics, 111(3):034907.

Moiseyenko, R. P., Liu, J., Declercq, N. F., and Laude, V. (2013). Blazed phononic crystal grating. Applied Physics Letters, 102(3):034108.

Pérez-Arjona, I., Sánchez-Morcillo, V., Redondo, J., Espinosa, V., and Staliunas, K. (2007). Theoretical prediction of nondiffractive propagation of sonic waves through periodic acoustic media. Physical Review B, 75:014304-1-014304-7.

Psarobas, I. E. (2001). Viscoelastic response of sonic band-gap materials. Phys. Rev. B, 64:012303.

Psarobas, I. E. and Sigalas, M. M. (2002). Elastic band gaps in a fcc lattice of mercury spheres in aluminum. Phys. Rev. B, 66:052302.

Qiu, C., Zhang, X., and Liu, Z. (2005). Far-field imaging of acoustic waves by a twodimensional sonic crystal. Physical Review B, 71(5):054302.

Robertson, W. and Rudy III, J. (1998). Measurement of acoustic stop bands in twodimensional periodic scattering arrays. The Journal of the Acoustical Society of America, 104(2):694-699.

Romero-García, V., Sánchez-Pérez, J., and Garcia-Raffi, L. (2011). Tunable wideband bandstop acoustic filter based on two-dimensional multiphysical phenomena periodic systems. Journal of applied physics, 110(1):014904.

Romero-García, V., Sánchez-Pérez, J. V., and García-Raffi, L. M. (2010). Evanescent modes in sonic crystals: Complex dispersion relation and supercell approximation. Journal of Applied Physics, 108(4):-.

Sánchez-Morcillo, V. J., Staliunas, K., Espinosa, V., Pérez-Arjona, I., Redondo, J., and Soliveres, E. (2009). Propagation of sound beams behind sonic crystals. Physical Review $B, 80: 134303-134303-7$.

Sánchez-Pérez, J. V., Caballero, D., Mártinez-Sala, R., Rubio, C., Sánchez-Dehesa, J., Meseguer, F., Llinares, J., and Gálvez, F. (1998). Sound attenuation by a two-dimensional array of rigid cylinders. Physical Review Letters, 80(24):5325-5328. 
Sanchis, L., Cervera, F., Sanchez-Dehesa, J., Sanchez-Perez, J., Rubio, C., and MartínezSala, R. (2001). Reflectance properties of two-dimensional sonic band-gap crystals. The Journal of the Acoustical Society of America, 109(6):2598-2605.

Shen, Y., Fu, J., and Yu, G. (2011). Rainbow trapping in one-dimensional chirped photonic crystals composed of alternating dielectric slabs. Physics Letters A, 375(43):3801 - 3803.

Sigalas, M. and Economou, E. (1992). Elastic and acoustic wave band structure. Journal of Sound and Vibration, 158(2):377-382.

Sigalas, M. and Economou, E. (1993). Band structure of elastic waves in two dimensional systems. Solid State Communications, 86(3):141-143.

Sigalas, M. M. (1997). Elastic wave band gaps and defect states in two-dimensional composites. Journal of Acoustical Society of America, 101(3):1256-1261.

Smolyaninova, V. N., Smolyaninov, I. I., Kildishev, A. V., and Shalaev, V. M. (2010). Experimental observation of the trapped rainbow. Applied Physics Letters, 96(21):-.

Staliunas, K. and Sánchez-Morcillo, V. J. (2009). Spatial filtering of light by chirped photonic crystals. Physical Review A: Atomic, Molecular, and Optical Physics, 79:053807-1-053807-6.

Sukhovich, A., Jing, L., and Page, J. H. (2008). Negative refraction and focusing of ultrasound in two-dimensional phononic crystals. Physical Review B, 77(1):014301.

Sukhovich, A., Merheb, B., Muralidharan, K., Vasseur, J. O., Pennec, Y., Deymier, P. A., and Page, J. H. (2009). Experimental and theoretical evidence for subwavelength imaging in phononic crystals. Physical Review Letters, 102:154301-154301-4.

Tanaka, Y., Tomoyasu, Y., and Tamura, S.-i. (2000). Band structure of acoustic waves in phononic lattices: Two-dimensional composites with large acoustic mismatch. Physical Review B, 62(11):7387.

Torrent, D., Håkansson, A., Cervera, F., and Sánchez-Dehesa, J. (2006). Homogenization of two-dimensional clusters of rigid rods in air. Physical review letters, 96(20):204302.

Torrent, D. and Sánchez-Dehesa, J. (2006). Effective parameters of clusters of cylinders embedded in a nonviscous fluid or gas. Physical Review B, 74(22):224305.

Tournat, V., Pagneux, V., Lafarge, D., and Jaouen, L. (2004). Multiple scattering of acoustic waves and porous absorbing media. Phys. Rev. E, 70:026609.

Umnova, O., Attenborough, K., and Linton, C. M. (2006). Effects of porous covering on sound attenuation by periodic arrays of cylinders. The Journal of the Acoustical Society of America, 119(1):278-284.

Wu, L.-Y. and Chen, L.-W. (2011). An acoustic bending waveguide designed by graded sonic crystals. Journal of Applied Physics, 110(11):-.

Wu, T.-T., Hsu, C.-H., and Sun, J.-H. (2006). Design of a highly magnified directional acoustic source based on the resonant cavity of two-dimensional phononic crystals. Applied physics letters, 89(17):171912.

Yablonovitch, E. (1987). Inhibited spontaneous emission in solid-state physics and electronics. Physical Review Letters, 58(20):2059-2062.

Yang, S., Page, J., Liu, Z., Cowan, M., Chan, C., and Sheng, P. (2004). Focusing of sound in a 3d phononic crystal. Physical review letters, 93(2):024301. 
Zhang, X. and Liu, Z. (2004). Negative refraction of acoustic waves in two-dimensional phononic crystals. Applied Physics Letters, 85(2):341-343.

Zigoneanu, L., Popa, B.-I., and Cummer, S. A. (2011). Design and measurements of a broadband two-dimensional acoustic lens. Physical Review B, 84(2):024305. 


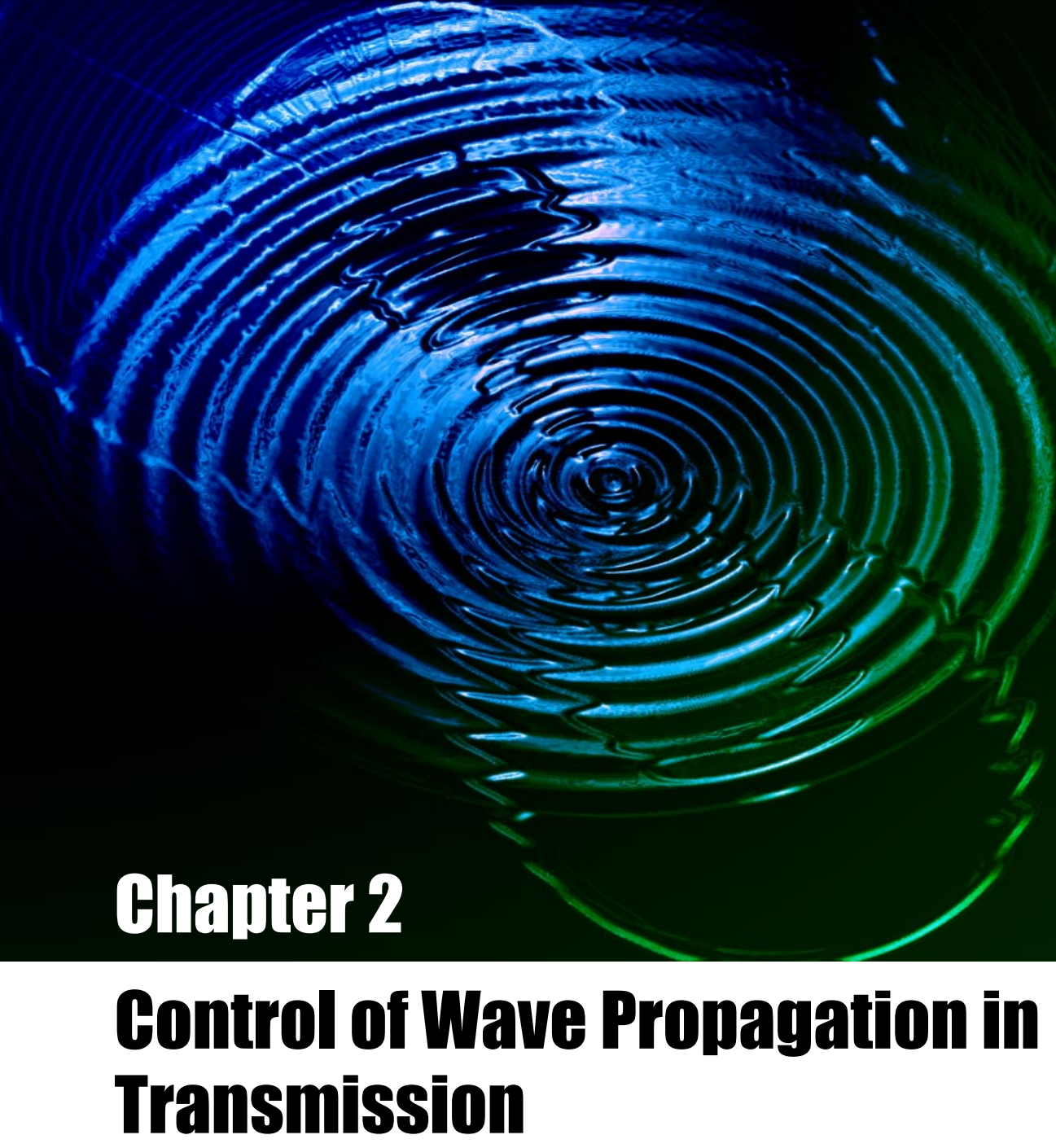

The contents of this chapter are organized in two sections. Section 2.1 is devoted to the study of focusing and collimation of beams in sonic crystals, describing the main concepts and methods used in "Formation of collimated sound beams by three-dimensional sonic crystals", published in Journal of Applied Physics. Section 2.2 encompass the design and modelling of a focusing device based on an axisymmetric gradient index (GRIN) lens working in the long wavelength regime, a work published in Applied Physics Letters under the title "Wave focusing using symmetry matching in axisymmetric acoustic gradient index lenses". In this chapter summary we describe some methods and concepts used in the aforementioned publications by means of numerical calculations. For further details and experimental results the reader is referred to Appendix A.1. 
Since the 40's ultrasonic sources became a main tool on industrial applications, since they constitute a noninvasive, nondestructive and low-cost exploration method. These have been used for the detection of inhomogeneities in different materials such as metals, plastics and wieldings, as well as to analyze its physical properties or thickness. Sonar technology was developed during World War II and initially used for remote sensing in underwater acoustics, although an extensive variety of underwater acoustics applications have emerged ever since, such as biomass estimation and bathymetry. In the 60's the use of ultrasound devices for medical imaging and diagnosis began and have been intensively improved, mainly due to microprocessors and digital signal processing, available since the 80's.

The increase on the use of ultrasonic sources in several branches of science and technology triggered the need to control and improve the radiation pattern of gaussian beam transducers [Huang and Breazeale, 2006], i.e. beamwidth and directivity, reduction of side lobes, beam direction, etc. Several active and passive methods were developed to achieve this goal. On one side, active methods featuring phased arrays have received much attention and several commercial devices for medical ultrasound have been developed. A phased array is formed by an array of elementary transducers controlled individually and electronically, where the application of suitable temporal delays on the excitation of each of the elements allow to focus or deflect the acoustic beam. On the other hand, passive methods such as the use of acoustic lenses [Shield and Harris, 1984], curved transducers or horns have been demonstrated as efficient tools to reduce the focusing distance and increase the gain. While the use of active methods offers higher flexibility than passive ones on the design of the radiation pattern of the acoustic source, specially for a high number of elements, phased arrays are expensive and its cost increases dramatically with the number of elements.

In the last few decades, it has become apparent that phononic/sonic crystals, i.e., materials whose physical properties are modulated in space, might constitute a great candidate to improve the focusing performance of passive methods, by modifying the spatial dispersion of propagating waves.

Among the various effects on wave propagation related to periodic structures, controlling the diffraction of waves has been an intensive area of research for the last decades. Different mechanisms allow to modify the spatial dispersion of propagating waves. Specifically, the analysis of the curves of equal frequency in $\vec{k}$-space (spatial dispersion or isofrequency curves) shows 
that beams can propagate within the periodic structure without diffraction (the so called self-collimation effect) due to the appearence of flat segments of the spatial dispersion curves. This effect has been predicted and experimentally demonstrated in acoustics [Pérez-Arjona et al., 2007, Soliveres et al., 2009] and optics [Zengerle, 1987].

Moreover, modification of spatial dispersion curves can produce interesting beaming phenomena not only inside a periodic structure, but outside of it, for finite-size structures. The negative refraction effect, reported firstly in optics [Pendry, 2000, Martínez et al., 2006, Bulu et al., 2003], has been intensively studied also in acoustics [Sukhovich et al., 2008, Zhang and Liu, 2004, Yang et al., 2004, Bucay et al., 2009], and allows to focus the waves outside the periodic structure, prompting potential applications based on lensing [Ke et al., 2005, Li et al., 2006, Qiu et al., 2005] and superlensing effects [Luo et al., 2003, Sukhovich et al., 2009, Zhang and Liu, 2008, Ke et al., 2007a].

Other approaches have been reported to obtain highly directional sources using periodic structures. Using the high density of states of propagating waves near the band-edge frequency, [Qiu and Liu, 2006, Ke et al., 2007b] or by resonant cavity modes operating at the band gap frequency [Hsu et al., 2008, Wu et al., 2006, Ke et al., 2006], the directional emission of point and linear (flat piston) acoustic sources has been substantially improved.

In most of real-world applications, such as in medical ultrasound [Baac et al., 2012], commercial ultrasonic transducers in sonar applications, aeroacoustics [Samimy et al., 2010], microfluidics [Choe et al., 2011], etc, acoustic wave sources usually posses radial symmetry. Hence, the symmetry matching between the source and the potential focusing device becomes a main feature of the source-lens system .

From a theoretical point of view, axisimmetric structures, i.e. structures posessing axial simmetry, cannot be considered as periodic structures since translational symmetry is lost, i.e., the wave equation is not invariant under periodic translations when an axisymmetric system is considered. This can be easily understood by considering the projection onto a $2 \mathrm{D}$ plane of periodic structure formed by a squared array of infinite length cylinders. An axial rotation of the previous $2 \mathrm{D}$ structure will generate a $3 \mathrm{D}$ axisimmetric structure and periodicity will be maintained in the radial and axisimmetric axis, but axial rotation will break periodicity in the $3^{\text {rd }}$ dimension. In this situation, Bloch theory is no longer applicable, isofrequency contours cannot be calculated and the curvature of the wave front along the focusing device 
cannot be estimated under this procedure.

A different approach used to overcome this limitation consists on working in the long wavelength regime. The ratio between wavelength of the incident wave, $\lambda$, and lattice constant, $a$, defines the working regime. For $\lambda>4 a$ periodic structures can be considered as an equivalent homogeneous medium with effective properties [Torrent et al., 2006], which are characterized by the theory of homogenization [Peng et al., 2010, Mei et al., 2006], allowing the study of the wave front profile in terms of refraction. Gradient index lenses, reported in 2D structures under a cartesian system of reference [Lin et al., 2009,Zigoneanu et al., 2011,Li et al., 2012], are excellent candidates to control wave propagation. These type of lenses, in an axisymmetric configuration, may improve transmission efficiency due to symmetry matching of the sourcelens system.

\subsection{Focusing and collimation of acoustic waves by periodic structures}

The focusing of waves behind a sonic crystal is related to the negative diffraction experienced by the wave inside the periodic structure. The character of beam propagation behind the crystal depends on the wave front of the beam acquired in the system. Hence, if the wave front acquires positive curvature due to propagation in a material with negative or anomalous diffraction, the beam can be focused behind the structure, where the positive diffraction in the homogeneous medium will compensate the accumulated phase differences at some distance. Fig 2.1 illustrates different types of diffraction according to the components of the wave vector $\vec{k}=\left(k_{x}, k_{y}\right)$ assuming wave propagation in $+x$ direction.

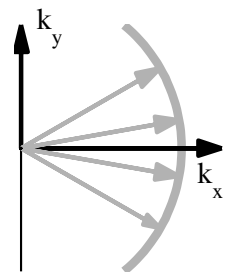

(a) Positive diffraction

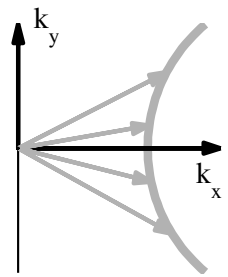

(b) Negative diffraction

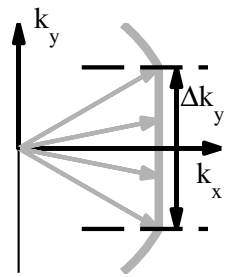

(c) Zero diffraction

Figure 2.1: $k$-space illustrating positive, zero and negative diffraction. 
Apart from the curvature of the wave front inside the periodic structure, the character of the source plays a key role on the focusing behavior. Specifically, the spatial spectrum of the source is a key parameter to take into account, as it represents the angular components of the incident beam. Point sources emit ominidirectional waves and thus radiate the same amplitude for all angles, whereas for linear sources, such as a flat piston emmitting acoustic beams, the spatial spectrum depends on its length. Qualitatively, a flat piston with an increasing length result in a narrower angular spectrum. This is observed in Fig. 2.2, where intensity field distributions and angular spectrum are shown for two flat pistons sources of lengths $D_{1}=2 a$ and $D_{2}=8 a$, where $a=5.25 \mathrm{~mm}$. The angular spectrum show a main lobe and several side-lobes of decreasing amplitude for increasing angles for both sources, although the width of the main lobe, where most of the energy is concentrated, is reduced when the size of the source is increased.

(a)

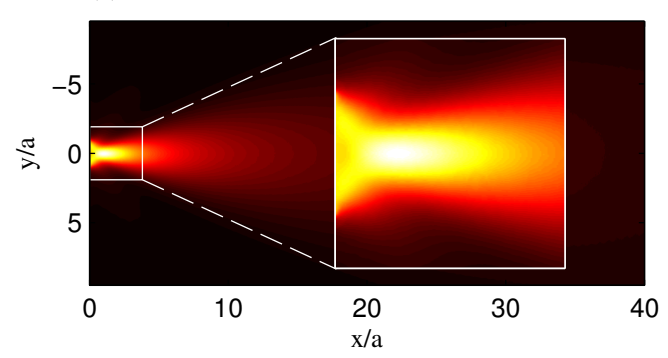

(c)

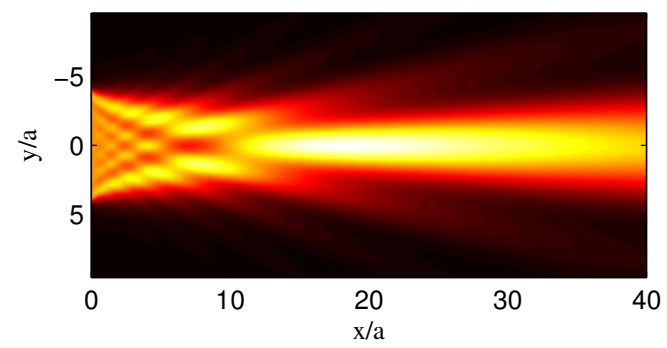

(b)

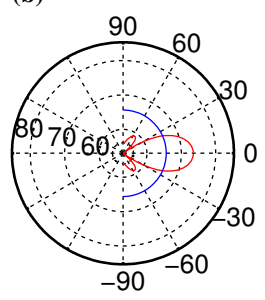

(d)

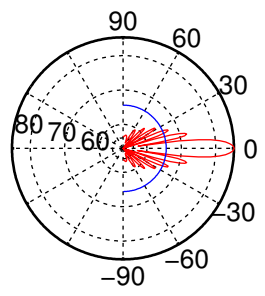

Figure 2.2: Intensity field distribution $\left(|p|^{2}\right)$ for $2 \mathrm{D}$ flat pistons of length (a) $D_{1}=2 a$, (c) $D_{2}=8 a$. (b), (d) Angular spectrum in $\mathrm{dB}$ for both sources (red lines) and an ideal point source (blue line), as a reference.

Circular pistons are commonly characterized in terms of its field distribution in space, i.e. near field distance, beamwidth at the waist, focal spot length, etc. However, in the context of beam formation using periodic struc- 
tures, the interplay between the spatial spectrum of the beam in the homogeneous and periodic media is crucial to succesfully predict the focusing performance of the source-lens system. This interplay is now described in terms of the curvature of equifrequency contours and the concept of anomalous diffraction. Besides, a simplified paraxial approximation, described in detail in previous work from our research group [Sánchez-Morcillo et al., 2009], is used to predict the focusing performance of a 2D finite-size SC, showing the focusing distance, $z_{f}$, as well as beamwidth at the focusing point, $B_{w}$ for the aforementioned flat pistons.

\subsubsection{Positive, zero and negative diffraction: Isofrequency con- tours}

As the first step, band structure and spatial dispersion curves are calculated by means of the Plane Wave Expansion Method (PWE). The inhomogeneous wave equation for spatially modulated medium is solved by formulating an eigenvalue problem and using Bloch boundary conditions, which allows to reduce the geometry to a unit cell. A square lattice SC is considered to illustrate the focusing mechanism. Fig 2.3 illustrates band structure for the main directions of propagation, i.e. at the edges of the Irreducible Brillouin Zone (IBZ) and the spatial dispersion curves for the $2^{\text {nd }}$ band of a SC consisting of a periodic arrangement of steel cylinders embedded in water with radius $r=0.8 \mathrm{~mm}$ and lattice constant $a=5.25 \mathrm{~mm}$. The resulting filling factor is $f=\pi r^{2} / a^{2}=0.073$. Thus, frequencies of interest are ranged in the ultrasonic regime, although this results are scalable to others range of frequencies by simply changing the geometrical parameters of the crystal. Material parameters are density $\rho_{w}=1000 \mathrm{~kg} / \mathrm{m}^{3}, \rho_{s}=7.800 \mathrm{~kg} / \mathrm{m}^{3}$ and speed of sound $c_{w}=1485 \mathrm{~m} / \mathrm{s}, c_{s}=4530 \mathrm{~m} / \mathrm{s}$.

Gradient vectors of the isofrequency contours in k-space define the group velocity vectors and hence, the propagation direction of the acoustic energy for a given wave component, $\vec{v}_{g}=\nabla_{k} \omega(k)$. All three possible types of diffraction are encountered in the spatial dispersion curves of the $2^{\text {nd }}$ band depicted in Fig 2.3(b). According to the previous definition and the negative slope of the $2^{\text {nd }}$ band, a negative curvature is found for isofrequency contours for frequencies ranging from $150-200 \mathrm{kHz}$, resulting in the regular positive diffraction of waves. An acoustic wave propagating within the SC at these frequencies will diverge as it would do in an homogeneous medium. Nonetheless, curvatures of these spatial dispersion curves are slightly deviated from a perfect circle, 

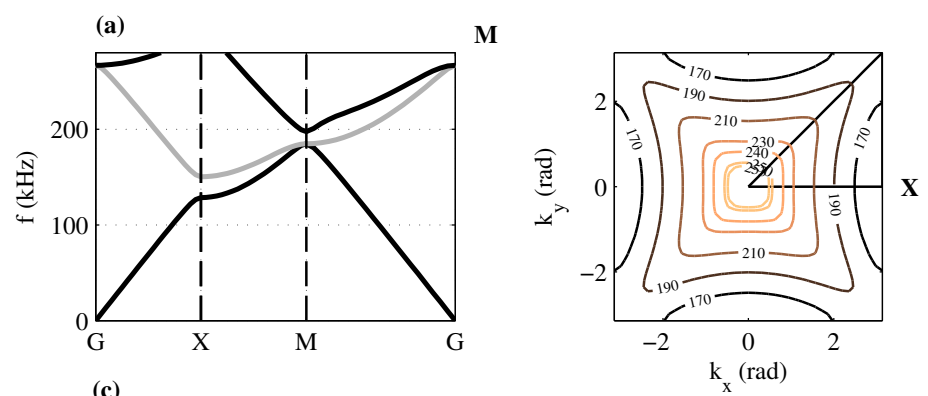

(c)

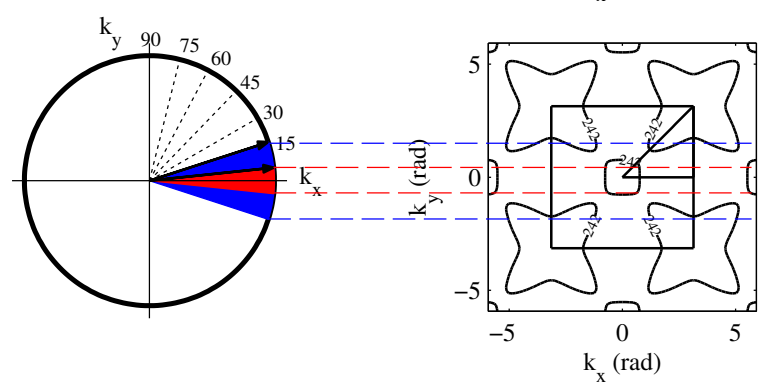

Figure 2.3: Graphical interpretation of the interplay between the incident wave generated by circular pistons $D_{1}, D_{2}$ and the periodic structure. Band structure (a) and isofrequency contours for the second band (b) of a square lattice SC consisting of steel cylinders embedded in water with radius $r=0,8 \mathrm{~mm}, a=5,25 \mathrm{~mm}$. (c) Illustrates the angular spectrum of both acoustic sources. (d) Spatial dispersion curves for $2^{\text {nd }}$ and $3^{\text {rd }}$ bands represented in an extended scheme. Dashed red and bluen lines relate the angular spectrum of the incident waves to the spatial dispersion curves.

characteristic of diffraction in a homogeneous medium, which means that the phase delay between component at high angles and low angles is lower and the wave will spread at a lower rate than it would do in water, for instance. The case is different for frequencies in the range $230-260 \mathrm{kHz}$, where the positive curvature of isofrequency contour result in a negative or anomalous diffraction of waves inside the crystal. Wave components close to zero accumulate some phase delay with respect to higher angular components, and the resulting phase profile of the wave acquire a negative curvature. A wave of this frequency propagating within the SC accumulates some phase shift, i. e. a negatively curved phase profile. As soon as it overcomes the structure and return to the host medium this phase profile starts to compensate and all wave components will be mutually in phase again at some distance. The amount of accumulated phase shift is directly related with the focusing distance. For 
a given SC of length $L$, the accumulated phase shift will increase together with the curvature of the isofrequency contour and the propagation distance needed to compensate it.

Let's recall the aforementioned flat pistons of different size to interpret the interplay between the angular spectrum of the incident wave and the shape of the spatial dispersion curve inside the SC. The diagram in Fig. 2.3(d) shows the isofrequency contour for a longitudinal wave propagating in water at $242 \mathrm{kHz}$ and the corresponding isofrequency contour for $2^{\text {nd }}$ and $3^{\text {rd }}$ bands in an extended scheme. Shaded blue and red areas in 2.3(c) indicate the angular spectrum content of the main lobes of flat pistons $D_{1}$ and $D_{2}$, being the Full width at half maximum (FWHM) of $18^{\circ}$ and $5^{\circ}$, respectively. The broad source $D_{2}$ posses a narrow angular spectrum and its main lobe fits completely within the corresponding contour inside the SC. Consequently, every angular component of the incident wave propagating inside the SC is allowed to propagate. The specific curvature of the spatial dispersion curve will influence the focusing mechanism, as it will be explained in next section under the simplified paraxial aproximation terms, but a well formed beam is expected. In opposition to that, Source $D_{1}$ accounts for a broader angular spectrum than the spatial dispersion curve of the $2^{\text {nd }}$ band for $242 \mathrm{kHz}$. Focusing is affected in this case by spatial dispersion curves belonging to neighboring bands ( $3^{\text {rd }}$ band in $\Gamma M$ direction) and also by angular band gaps [Picó et al., 2013, Romero-García et al., 2013]. These are angular areas where waves are not allowed to propagate. As a result, those angular components are filtered out from the beam as it propagates through the SC, the beam quality and amplitude are affected and a focusing with a significant distortion of the spatial spectrum of the beam is predicted.

\subsubsection{Paraxial approximation}

The shape of the above described spatial dispersion curves allow to determine the leading and higher order terms of coefficients of diffraction. Assuming a small filling factor, for frequencies close to the band gap and considering wave propagation in $+x$ direction, the isofrequency curve $k_{x}=k_{x}\left(k_{y}\right)$ can be expanded in series around $k_{y}=0$ [Sánchez-Morcillo et al., 2009]:

$$
k_{x}=d_{0}+d_{2} k_{y}^{2}+d_{4} k_{y}^{4}+\ldots
$$


where coefficients depend on the SC parameters such as frequency and filling factor

$$
\begin{aligned}
& d_{0}=\alpha \Delta \Omega+O(\varepsilon), \\
& d_{2}=\alpha\left(1-\frac{f^{2}}{\Delta \Omega^{3}}\right)+O(\varepsilon), \\
& d_{4}=2 \alpha\left(\frac{1}{\Delta \Omega^{2}}-\frac{f^{2}}{\Delta \Omega^{5}}\right)+O\left(\varepsilon^{-1}\right),
\end{aligned}
$$

where $f=\pi r^{2} / a^{2}$ is the filling fraction, $\alpha$ is a geometrical factor depending on the angle of the incident wave, being $\alpha=1$ and $\alpha=1 / \sqrt{2}$ for $\Gamma \mathrm{X}$ and $\Gamma \mathrm{M}$, respectively. $\varepsilon$ is the smallness parameter and indicates how far is a given spatial dispersion curve from the edge of Brillouin Zone. The detuning parameter $\Delta \Omega$ is defined as:

$$
\Delta \Omega=\left(\Omega_{g}-\Omega\right) / \Omega_{g},
$$

where $\Omega_{g}=\omega_{g} a / 2 \pi c_{h}$ is the normalized Bragg frequency, $a$ is the lattice constant and $c_{h}$ is the speed of sound in the host medium. The diffraction coefficients shown in Eq. (2.2) are obtained assuming the detuning parameter of order $\varepsilon$, and the filling fraction of order $\varepsilon^{2}$.

We now consider the case of a broad beam having narrow angular spectrum, similar to the one described previously for a flat piston of length $D_{2}=8 a$. The corresponding spatial dispersion curve in the $\mathrm{SC}$ is assumed to be completely parabolic and no higher order diffraction terms are considered $\left(d_{4} k_{y}^{4} \rightarrow 0\right)$, as stated in [Sánchez-Morcillo et al., 2009]. The acumulated negative phase shift of the components of the spatial spectrum in the SC, $\Delta \varphi\left(k_{x}\right)=-d_{2} k_{y}^{2} L$, are compensated at the focusing distance $z_{f}$, where the spatial components are all again in phase, i.e. $d_{2} L+z_{f}=0$. Hence, the beam will focus behind the $\mathrm{SC}$ at some distance depending on frequency:

$$
z_{f}=\alpha L\left(\frac{f^{2}}{\Delta \Omega^{3}}\right),
$$

where $L$ is the length of the SC.

Fig. 2.4 illustrates numerical results. Focusing distance $z_{f}$ is shown in Fig. 2.4(c), where on-axis absolute value of pressure depending on frequency is depicted in density plot for $D_{2}=8 a$. Analytical prediction (continuous grey line) following Eq. (2.4) and numerical results(black crosses) are in good 
(a) $\quad \mathrm{D}_{2}=8 \mathrm{a} ; \quad|\mathrm{p}|^{2} ; \mathrm{f}=242 \mathrm{kHz}$

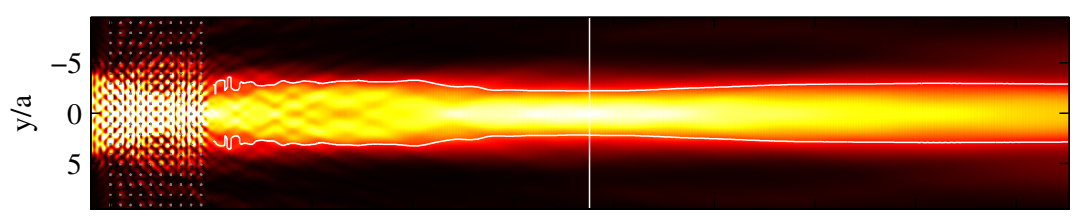

(b)

$\mathrm{D}_{1}=2 \mathrm{a} ; \quad|\mathrm{p}|^{2} ; \quad \mathrm{f}=242 \mathrm{kHz}$
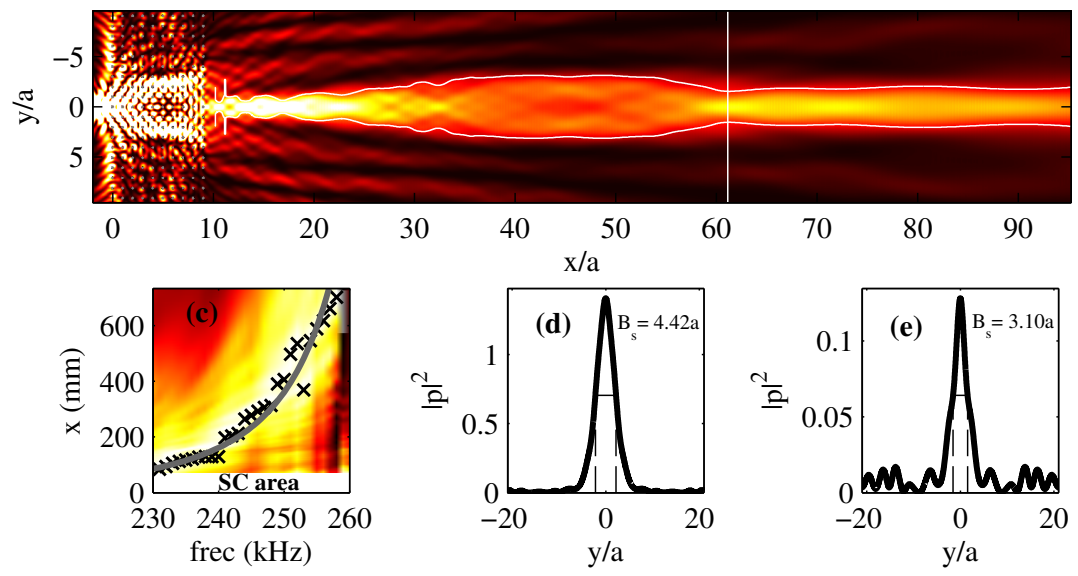

Figure 2.4: Numerical results. Intensity field distributions obtained for a broad (a) and narrow (b) beam for a frequency $f=242 \mathrm{kHz}$. White continuous line indicates the focusing distance $z_{f}$ calculated using Eq. (2.4). (c) On-axis absolute value of the pressure $(|p|)$ behind the SC for frequencies corresponding to the $2^{\text {nd }}$ band. The acoustic field is normalized to the maximum value along the axis (represented by black crosses) for each frequency in order to track the focusing point. Grey continuous line represent the analytical prediction from Eq. (2.4). Tranversal cuts showing the width of the beam at the focus point are included for broad (d) and narrow beams (e).

agreement. Moreover, a well focused beam is formed for the broad beam in Fig. 2.4(a), as predicted previously from analysis of isofrequency contours. If a narrow gaussian beam is considered, such as the flat piston of length $D_{1}=2 a$, the situation is different. Angular components corresponding to small angles still play their role and accordingly, the beam is focused behind the SC. However, beam profile is now affected due to non-parabolic phase shift of high angular components, resulting in aberrations. Moreover, angular band gaps also come into play and those angular components within this spectral area are removed. This results in a complicated field distribution, the beam at its waist presents several oscillations related with the interference 
between the central spectral component and the sidebands, as illustrated in Fig. 2.4(e). Besides, a modulated profile is also observable in the main axis of propagation, where several focuses are apparent, as shown in Fig. 2.4(b).

As stated previously, real acoustic sources are far from ideal point sources radiating omnidirectional waves or infinite length linear sources radiating plane waves, behaving as flat pistons with intensity field distributions similar to those shown in Fig. 2.2. When working on 3D systems, the absence of symmetry between a $3 \mathrm{D}$ periodic structure and a real $3 \mathrm{D}$ acoustic source having axial symmetry reduces considerably the efficiency of the lensing system in terms of gain. Such a case is depicted in Fig. 3(b) in "Formation of collimated sound beams behind 3D sonic crystals" (see Appendix A.1), where the measured beam amplitude at the focal point, normalized to the amplitude of the same source in the absence of SC, is depicted. With the aim of overcoming this drawback we design and test a 3D axisimmetric structure capable of subtantially increasing the gain of the source-lens system compared to a regular $3 \mathrm{D}$ periodic structure.

\subsection{Wave focusing on axisymmetric structures}

The study of focusing mechanisms on periodic structures in two and three dimensions has been mainly devoted to cartesian systems. This type of systems, i.e., square or triangular lattices in $2 \mathrm{D}$ or cubic lattices in $3 \mathrm{D}$, are best described by $(\mathrm{x}, \mathrm{y}),(\mathrm{x}, \mathrm{y}, \mathrm{z})$ coordinates and translational symmetry is guaranteed. Hence, Bloch theory based methods used to characterize wave propagation in periodic structures are of application. As the next step to improve the focusing performance of potential devices based in periodic structures, one may think on the design of $3 \mathrm{D}$ structures having axial symmetry in order to match the radial symmetry of acoustic sources.

Let's have a look to the wave equation in cartesian and cylindrical coordinates in order to compare the systems represented in Figs. 2.5(a)-(b). Assuming harmonic time dependence the wave equation for acoustic waves reduce to an inhomogeneous Helmholtz equation:

$$
\nabla\left(\frac{1}{\rho} \nabla p\right)+\frac{\omega^{2}}{\rho c^{2}} p=0 .
$$

Depending on to the geometry of the problem the fluid is characterized by its density $\rho(x, y), \rho(r, z)$, and speed of sound $c(x, y), c(r, z)$, which are 

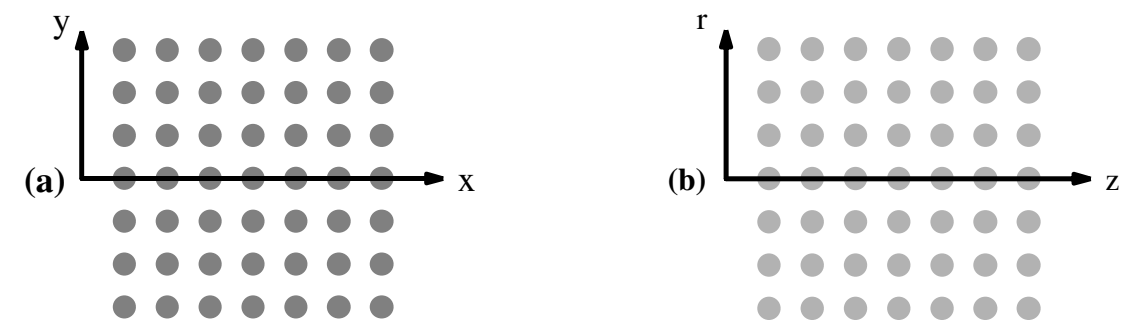

Figure 2.5: (a) Projection onto a 2D plane of a periodic system made of infinite length cylinders represented in cartesian coordinates. (b) Cut at $\phi=0$ of an axial symmetric $3 \mathrm{D}$ structure represented using a cylindrical coordinate system. Filled circles represent the geometry of a square lattice of some material in a host domain.

periodic functions of space expressed in the $x y, r z$ planes, respectively. Considering the Laplace operator in Cartesian coordinates, Eq. (2.5) yields:

$$
\frac{\partial}{\partial x}\left(\frac{1}{\rho(x, y)} \frac{\partial p}{\partial x}\right)+\frac{\partial}{\partial y}\left(\frac{1}{\rho(x, y)} \frac{\partial p}{\partial y}\right)+\frac{\omega^{2}}{\rho(x, y) c(x, y)^{2}} p=0
$$

which is invariant under translations defined by the lattice parameter of the crystal $a$. For the square lattice depicted in Fig. 2.5(a), Eq. (2.6) is invariant under translations $x \rightarrow x+n a, y \rightarrow y+n a$, and so are the physical parameters of the system. We consider now Eq. (2.5) taking the Laplace operator in cylindrical coordinates, as in the system in Fig. 2.5(b):

$$
\frac{1}{r} \frac{\partial}{\partial r}\left(\frac{r}{\rho(r, z)} \frac{\partial p}{\partial r}\right)+\frac{\partial}{\partial z}\left(\frac{1}{\rho(r, z)} \frac{\partial p}{\partial z}\right)+\frac{\omega^{2}}{\rho(r, z) c(r, z)^{2}} p=0
$$

Using this system of reference the wave equation is invariant under translations $z \rightarrow z+n a$, but it is not under translations of the form $r \rightarrow r+n a$ due to the presence of $r$ and $\frac{1}{r}$ in the first term of Eq. (2.7) despite the fact that the physical properties of the system in Fig. 2.5(b) are indeed periodic functions for a $2 \mathrm{D}$ system. A similar deduction can be made for the axisymmetric version of Eq. (2.7). In this case, i.e., applying an axial rotation to the system in Fig. 2.5(b), neither the wave equation nor the physical properties in the domain are invariant under transformations in the radial axis. As a direct consequence, an axisymmetric structure cannot be considered periodic and a different approach from Bloch Theory has to be considered. 


\subsubsection{Homogenization theory}

In the long wavelength regime $(\lambda>4 a)$, homogenization theory [Cervera et al., 2001, Krokhin et al., 2003] states that a periodic array of rigid inclusions in a fluid medium (host medium) can be considered as an equivalent homogeneous fluid having effective properties, such as effective density and sound velocity, which are determined by the filling fraction of the structure [Torrent et al., 2006].

Hence, the effective density of the medium and the effective velocity of waves propagating in an homogenized medium can be expressed in terms of the filling factor $f$ as:

$$
\rho_{\text {eff }}=\rho_{\text {host }} \frac{1+f}{1-f}, c_{e f f}=\frac{c_{\text {host }}}{\sqrt{1+f}} .
$$

Another important feature of the medium is its acoustic impedance, defined as the product of the sound velocity $c$ and mass density $\rho$. Specifically, the impedance contrast between the host medium and the focusing device is directly related to the transmittance at the interface between the two media. According to the previous definitions of the effective properties of the homogenized medium, the acoustic effective impedance of the designed medium in the homogenization limit is defined as follows:

$$
Z_{\text {eff }}=Z_{\text {host }} \frac{\sqrt{1+f}}{1-f} .
$$

\subsubsection{Axisymmetric gradient index lens}

Our goal is the design of an axisymmetric gradient index lens formed by rigid toroidal scatterers embedded in air working with a circular piston radiating Gaussian beams in the long wavelength regime, considering waves having a wavelength $\lambda>4 a$, where $a$ is the distance between the center of neighbor scatterers. Taking into account the effective physical properties of the system in the homogenization regime, described previously, the index of refraction can be defined in terms of the effective velocity and filling fraction as:

$$
n(r)=\frac{c_{\text {host }}}{c_{\text {eff }}}=\sqrt{1+f(r)},
$$


where $f(r)=\pi R(r)^{2} / a^{2}$ is the filling fraction of the structure, which varies gradually along the radial axis according to the desired profile for the refraction index. Figure. 2.6 represent a schematic diagram of the axisymmetric structure. Each scatterer is represented by a major radius $R^{\prime}$, which is the radius of each toroid, and a minor radius $R$, which determines the filling fraction for a given distance between scatterers $a$.
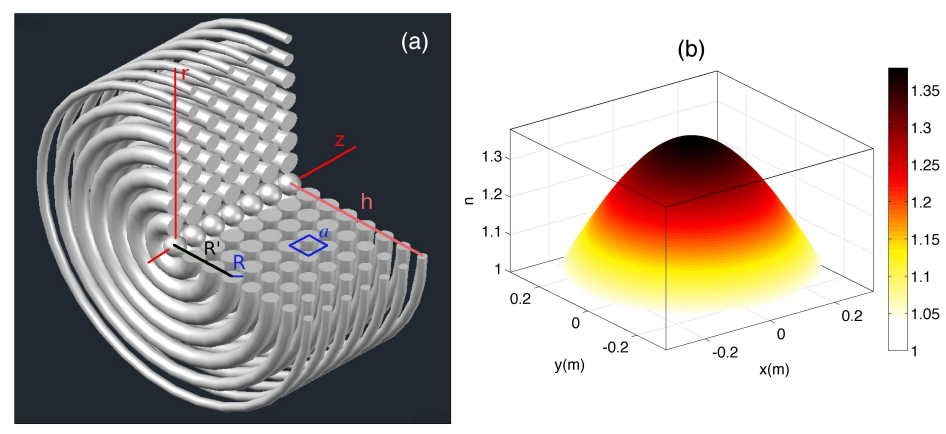

Figure 2.6: (a) Axisymmetric GRIN lens and its main geometrical parameters. (b) Refraction index profile used for the GRIN lens.

A hiperbolic secant profile that has been shown to reduce the aberration of the focal spot [Gomez-Reino et al., 2002] is chosen to define the index radial variation:

$$
n(r)=n_{0} \operatorname{sech}(\alpha r)
$$

where $n_{0}=n(r=0)$ is the refractive index along $z$-axis and $\alpha$ is the gradient coefficient:

$$
\alpha=\frac{1}{h} \cosh ^{-1}\left(\frac{n_{0}}{n_{h}}\right),
$$

where $h$ is the half-width of the lens and $n_{h}$ the refraction index at the lens edges $(r= \pm h)$. Figure 2.6(b) shows the refractive index profile where $n_{0}=1$ and $n_{h}=1.33$. The distance between neighbor scatterers is $a=0.04 \mathrm{~m}$ and $h=10 a$ the thickness of the lens. 7 planes of toroidal scatterers were used, as shown in Fig. 2.6(a).

In order to validate the selected approach we compare numerical results calculated using the Finite Element Method for the axisymmetric structure versus an homogeneous fluid whose physical properties, i.e., sound velocity and density have been defined to fit the refraction index profile shown in Fig. 
2.6(b). Figure. 2.7 represent the acoustic field (absolute value of pressure $|p|$ ) behind the axisymmetric structure, shown in Figs. 2.7(a), (b), versus the field behind an homogeneous equivalent fluid of the same size, shown in Figs. $2.7(\mathrm{c}),(\mathrm{d})$. The acoustic field is generated by a circular piston of diameter $D=5 a$ emitting waves at two different wavelengths $\lambda=7 a$ and $\lambda=4.25 a$. The source is placed at a distance $5 a$ from the structures. The acoustic field is modulated in amplitude due to Fabry-Pérot resonances related to the length of the lens $(L=7 a)$.
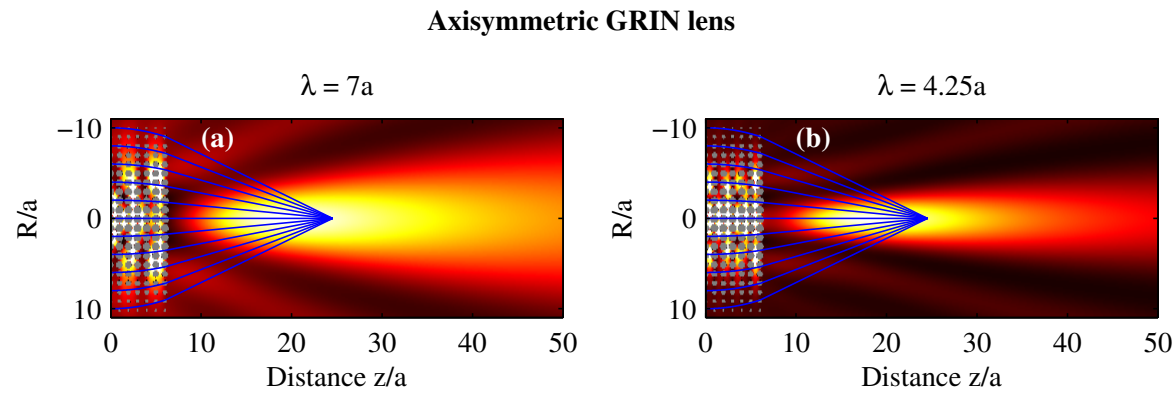

\section{Equivalent Fluid}
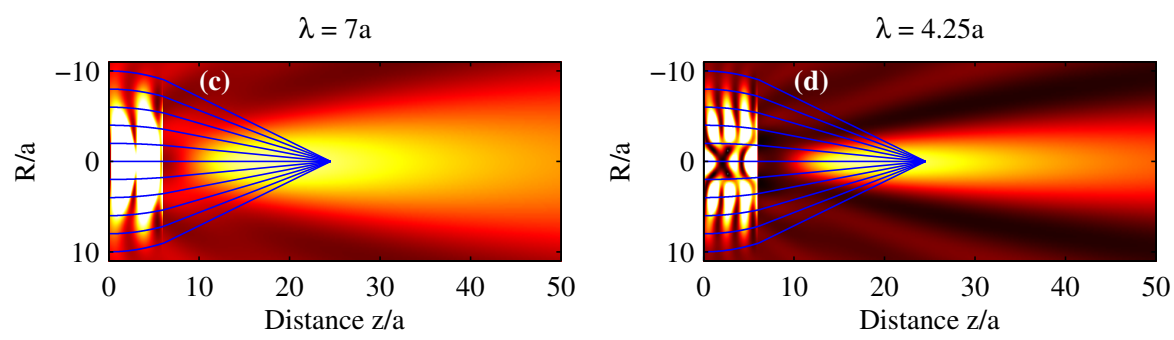

Figure 2.7: Comparison between the acoustic field in the $r z$-plane for the axisymmetric GRIN lens (a), (b) and the equivalent fluid lens (c), (d), for $\lambda=7 a, \lambda=4.25$. Blue continuous lines represent the ray-tracing trajectories calculated from the derivative of Eq. (2.13) at the interface and considering Snell's law of refraction.

The hyperbolic secant refractive index profile is used to determine the ray-tracing trajectories inside the GRIN medium as follows [Lin et al., 2009]:

$$
y(z)=\frac{1}{\alpha} \sinh ^{-1}\left(u_{0} H_{f}(z)+\frac{d u_{0}}{d z} H_{a}(z)\right),
$$

where $u_{0}=\sinh \left(y_{0}\right), H_{a}(z)=\sin (\alpha z) / \alpha$ and $H_{f}(z)=\cos (\alpha z)$. The deriva- 
tive of Eq. (2.13) at the interface of the GRIN lens, together with Snell's law of refraction allows to determine the focus position beyond the GRIN lens. Blue continuous lines in Fig. 2.7 represent the ray-tracing trajectories, calculated using Eq. (2.13) inside the samples and applying Snell's law at the interface between the two media. A good agreement is observed between numerical simulations for the equivalent fluid and the axisymmetric GRIN lens for frequencies close to the homogenization limit. Moreover, the predicted focal distance by ray-tracing trajectories is $z_{f} \approx 25 a$, matching well with numerical results, although some mismatch is found for $\lambda=4.25 a$ where the focal distance is slightly lower, which might be due to dispersive effects near the homogenization limit related to the high filling fraction defined at the center of the axisymmetric GRIN lens.

The sound amplification (SA) produced by the axisymmetric GRIN lens is calculated numerically and compared to the SA produced by a 2D GRIN lens designed with the same refraction index profile. The SA is evaluated at the focal point as $S A(d B)=10 \log _{10}\left(|p|_{\text {lens }}^{2} /|p|_{\text {free }}^{2}\right)$. A remarkable value of SA is obtained for the axisymmetric version of the GRIN lens, $S A_{3 D}=8.66 \mathrm{~dB}$, in contrast to the $2 \mathrm{D}$ version of the GRIN lens, $S A_{2 D}=4.81 \mathrm{~dB}$ and other reported results on the literature for Cartesian lenses [Climente et al., 2010,Martin et al., 2010]. This result confirms the relevance of the symmetry matching between the circular source and the axisymmetric structure increasing the focusing properties of the full source-lens system. Moreover, recalling the effective acoustic impedance shown in Eq. (2.9), it is noted that the maximum impedance contrast is found in the center of the lens, $Z_{\text {eff }}(r=0)=6.2 Z_{\text {host }}$, and its profile along the radial axis is governed by the refraction index. This impedance profile ensures that acoustic waves impinging the GRIN lens are weakly reflected and strongly refracted, reinforcing the high SA obtained using the designed GRIN lens.

Finally, the influence of the index of refraction, both $n_{0}$ and $\alpha$, in the focal distance is analyzed and represented in Figure 2.8 for $n_{0}=1.29, n_{0}=1.24$, and $\lambda=4.25 a$. Results from numerical simulations match well with the predicted focusing distance from ray-tracing trajectories, showing an increasing focusing distance as $n_{0}$ decreases. Some decrease in the focusing distance is found near the homogenization limit, indicating some dependence of the gradient coefficient with frequency. This influence is noticeable for higher values of $\alpha$, resulting in a lower focusing distance compared to ray-tracing, as observed in $2.8(\mathrm{a})$ 

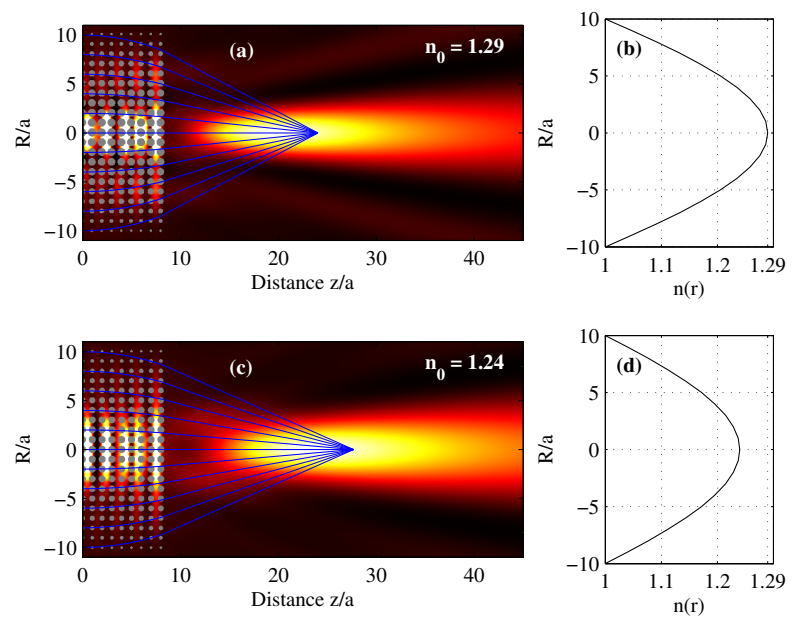

Figure 2.8: Acoustic field in the $r z$-plane showing the absolute value of the pressure $|p|$ for an axisymmetric GRIN lens for (a), $n_{0}=1.29$, (c) $n_{0}=1.24$. (c), (d) illustrate the corresponding refraction index profiles. Blue continuous lines represent the ray-tracing trajectories.

In section 2.1, main concepts and methods used in the publication included in Appendix A.1 have been explained in the context of a simple 2D geometry that allowed to extend the analysis showing different focusing regimes, which depend on the size of the source. The obtained results are interpreted and analyzed in terms of the curvatures of the spatial dispersion curves and are due to the negative diffraction close to the edge of the propagating band. Numerical results fit well with analytical predictions in Ref. [Sánchez-Morcillo et al., 2009], showing the tunability of the focusing distance.

Section 2.2 has been devoted to the model and design of an axisymmetric gradient index lens presenting a geometry matching with the acoustic source. The system is made of rigid toroidal scatterers embedded in air by varying the filling fraction in the radial axis in order to reproduce an hyperbolic secant profile. The ray-tracing trajectories, the effective fluid medium aproximation and the numerical prediction using the finite element method are all in good agreement showing a remarkable sound amplification which also agrees with the experimental results in Appendix A.2. The reader is referred to Appendices A.1, A.2 for further details and experimental results. 


\section{References}

Baac, H. W., Ok, J. G., Maxwell, A., Lee, K.-T., Chen, Y.-C., Hart, A. J., Xu, Z., Yoon, E., and Guo, L. J. (2012). Carbon-nanotube optoacoustic lens for focused ultrasound generation and high-precision targeted therapy. Scientific reports, 2.

Bucay, J., Roussel, E., Vasseur, J., Deymier, P. A., Hladky-Hennion, A.-C., Pennec, Y., Muralidharan, K., Djafari-Rouhani, B., and Dubus, B. (2009). Positive, negative, zero refraction and beam splitting in a solid/air phononic crystal: Theoretical and experimental study. Physical Review B, 79:214305-214305-7.

Bulu, I., Caglayan, H., and Ozbay, E. (2003). Highly directive radiation from sources embedded inside photonic crystals. Applied physics letters, 83(16):3263-3265.

Cervera, F., Sanchis, L., Sanchez-Perez, J., Martinez-Sala, R., Rubio, C., Meseguer, F., López, C., Caballero, D., and Sánchez-Dehesa, J. (2001). Refractive acoustic devices for airborne sound. Phys. Rev. Lett., 88:023902.

Choe, Y., Kim, J. W., Shung, K. K., and Kim, E. S. (2011). Microparticle trapping in an ultrasonic bessel beam. Applied physics letters, 99(23):233704.

Climente, A., Torrent, D., and Sánchez-Dehesa, J. (2010). Sound focusing by gradient index sonic lenses. Applied Physics Letters, 97(10):104103.

Gomez-Reino, C., Perez, M. V., and Bao, C. (2002). Gradient-index optics: fundamentals and applications. Springer.

Hsu, F.-C., Wu, T.-T., Hsu, J.-C., and Sun, J.-H. (2008). Directional enhanced acoustic radiation caused by a point cavity in a finite-size two-dimensional phononic crystal. Applied Physics Letters, 93(20):201904.

Huang, D. and Breazeale, M. A. (2006). An ultrasonic gaussian transducer and its diffraction field: theory and practice. Ultrasonics, Ferroelectrics, and Frequency Control, IEEE Transactions on, 53(5):1018-1027.

Ke, M., Liu, Z., Cheng, Z., Li, J., Peng, P., and Shi, J. (2007a). Flat superlens by using negative refraction in two-dimensional phononic crystals. Solid State Communications, 142(3):177-180.

Ke, M., Liu, Z., Pang, P., Qiu, C., Zhao, D., Peng, S., Shi, J., and Wen, W. (2007b). Experimental demonstration of directional acoustic radiation based on two-dimensional phononic crystal band edge states. Applied physics letters, 90(8):083509.

Ke, M., Liu, Z., Pang, P., Wang, W., Cheng, Z., Shi, J., Zhao, X., and Wen, W. (2006). Highly directional acoustic wave radiation based on asymmetrical two-dimensional phononic crystal resonant cavity. Applied physics letters, 88(26):263505.

Ke, M., Liu, Z., Qiu, C., Wang, W., Shi, J., Wen, W., and Sheng, P. (2005). Negative-refraction imaging with two-dimensional phononic crystals. Physical Review B, 72(6):064306.

Krokhin, A., Arriaga, J., and Gumen, L. (2003). Speed of sound in periodic elastic composites. Physical review letters, 91(26):264302.

Li, J., Liu, Z., and Qiu, C. (2006). Negative refraction imaging of acoustic waves by a two-dimensional three-component phononic crystal. Physical Review B, 73(5):054302. 
Li, Y., Liang, B., Tao, X., Zhu, X.-f., Zou, X.-y., and Cheng, J.-c. (2012). Acoustic focusing by coiling up space. Applied Physics Letters, 101(23):233508.

Lin, S.-C. S., Huang, T. J., Sun, J.-H., and Wu, T.-T. (2009). Gradient-index phononic crystals. Physical Review B, 79(9):094302.

Luo, C., Johnson, S. G., Joannopoulos, J. D., and Pendry, J. B. (2003). Subwavelength imaging in photonic crystals. Physical Review B, 68:045115-045115-14.

Martin, T. P., Nicholas, M., Orris, G. J., Cai, L.-W., Torrent, D., and Sánchez-Dehesa, J. (2010). Sonic gradient index lens for aqueous applications. Applied Physics Letters, 97(11):113503.

Martínez, A., Piqueras, M. A., and Martí, J. (2006). Generation of highly directional beam by k-space filtering using a metamaterial flat slab with a small negative index of refraction. Applied physics letters, 89(13):131111.

Mei, J., Liu, Z., Wen, W., and Sheng, P. (2006). Effective mass density of fluid-solid composites. Physical review letters, 96(2):024301.

Pendry, J. B. (2000). Negative refraction makes a perfect lens. Physical review letters, 85(18):3966.

Peng, S., He, Z., Jia, H., Zhang, A., Qiu, C., Ke, M., and Liu, Z. (2010). Acoustic far-field focusing effect for two-dimensional graded negative refractive-index sonic crystals. Applied Physics Letters, 96(26):263502.

Pérez-Arjona, Sánchez-Morcillo, V., Redondo, J., Espinosa, V., and Staliunas, K. (2007). Theoretical prediction of nondiffractive propagation of sonic waves through periodic acoustic media. Physical Review B, 75:014304-1-014304-7.

Picó, R., Pérez-Arjona, I., Sánchez-Morcillo, V., and Staliunas, K. (2013). Evidences of spatial (angular) filtering of sound beams by sonic crystals. Applied Acoustics, 74(7):945-948.

Qiu, C. and Liu, Z. (2006). Acoustic directional radiation and enhancement caused by band-edge states of two-dimensional phononic crystals. Applied physics letters, 89(6):063106.

Qiu, C., Zhang, X., and Liu, Z. (2005). Far-field imaging of acoustic waves by a twodimensional sonic crystal. Physical Review B, 71(5):054302.

Romero-García, V., Picó, R., Cebrecos, A., Staliunas, K., and Sánchez-Morcillo, V. J. (2013). Angular band gaps in sonic crystals: Evanescent waves and spatial complex dispersion relation. Journal of Vibration and Acoustics, 135(4):041012.

Samimy, M., Kim, J.-H., Kearney-Fischer, M., and Sinha, A. (2010). Acoustic and flow fields of an excited high reynolds number axisymmetric supersonic jet. Journal of Fluid Mechanics, 656:507-529.

Sánchez-Morcillo, V. J., Staliunas, K., Espinosa, V., Pérez-Arjona, I., Redondo, J., and Soliveres, E. (2009). Propagation of sound beams behind sonic crystals. Physical Review $B, 80: 134303-134303-7$.

Shield, T. W. and Harris, J. G. (1984). An acoustic lens design usign the geometrical theory of diffraction. Journal of Acoustical Society of America, 75(5):1634-1635. lensing, acoustics, diffraction, ray theory. 
Soliveres, E., Espinosa, V., Pérez-Arjona, I., Sánchez-Morcillo, V., and Staliunas, K. (2009). Self collimation of ultrasound beams in a three-dimensional sonic crystal. $A p$ plied Physics Letters, 94:164101-1-164101-3.

Sukhovich, A., Jing, L., and Page, J. H. (2008). Negative refraction and focusing of ultrasound in two-dimensional phononic crystals. Physical Review B, 77(1):014301.

Sukhovich, A., Merheb, B., Muralidharan, K., Vasseur, J. O., Pennec, Y., Deymier, P. A., and Page, J. H. (2009). Experimental and theoretical evidence for subwavelength imaging in phononic crystals. Physical Review Letters, 102:154301-154301-4.

Torrent, D., Håkansson, A., Cervera, F., and Sánchez-Dehesa, J. (2006). Homogenization of two-dimensional clusters of rigid rods in air. Physical review letters, 96(20):204302.

Wu, T.-T., Hsu, C.-H., and Sun, J.-H. (2006). Design of a highly magnified directional acoustic source based on the resonant cavity of two-dimensional phononic crystals. Applied physics letters, 89(17):171912.

Yang, S., Page, J., Liu, Z., Cowan, M., Chan, C., and Sheng, P. (2004). Focusing of sound in a 3d phononic crystal. Physical review letters, 93(2):024301.

Zengerle, R. (1987). Light propagation in singly and doubly periodic planar waveguides. Journal of Modern Optics, 34(12):1589-1617.

Zhang, X. and Liu, Z. (2004). Negative refraction of acoustic waves in two-dimensional phononic crystals. Applied Physics Letters, 85(2):341-343.

Zhang, X. and Liu, Z. (2008). Superlenses to overcome the diffraction limit. Nature materials, 7(6):435-441.

Zigoneanu, L., Popa, B.-I., and Cummer, S. A. (2011). Design and measurements of a broadband two-dimensional acoustic lens. Physical Review B, 84(2):024305. 


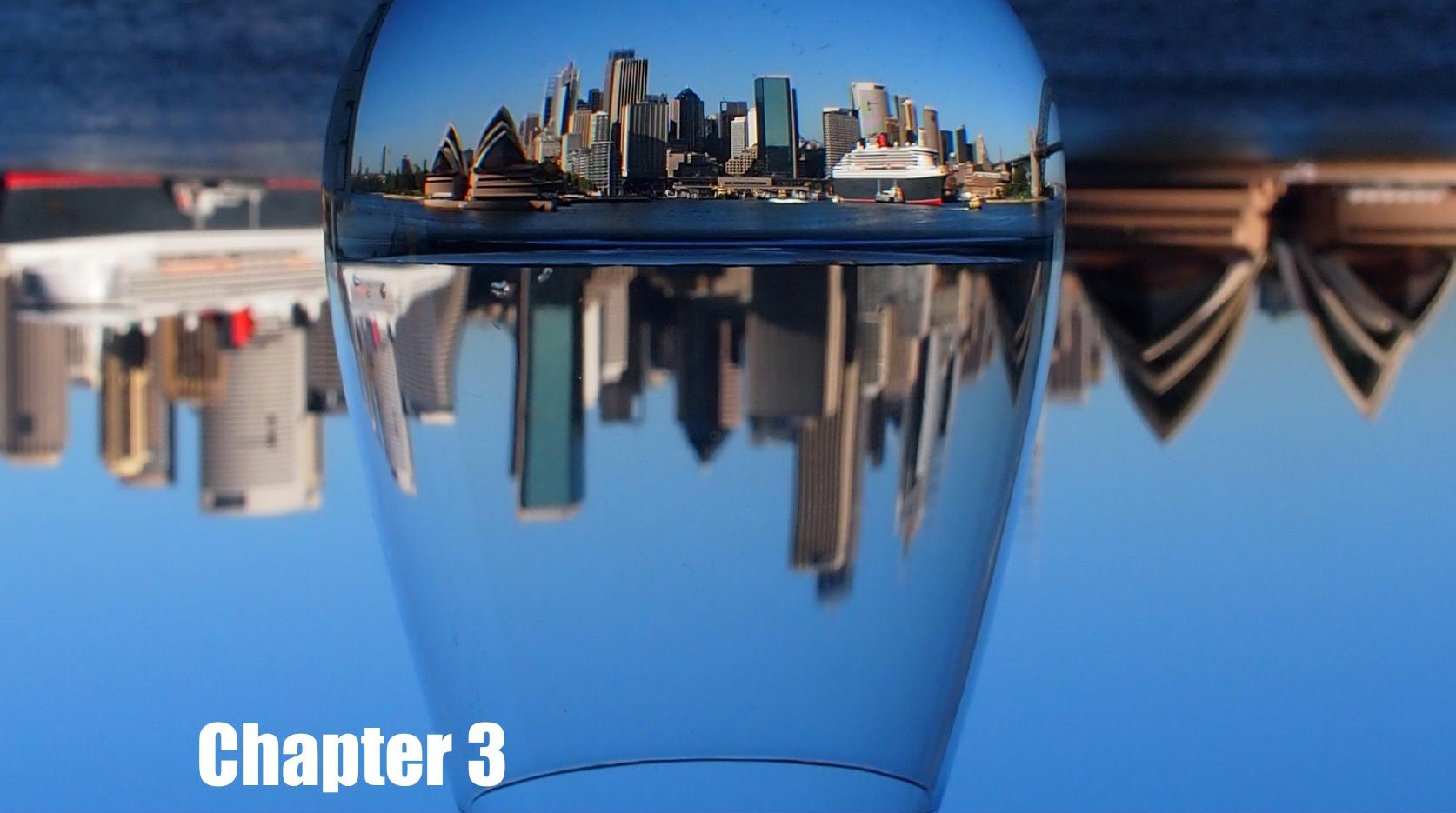

\section{Reflection in Chirped Sonic Crystals}

The contents of this chapter encompass the theory and main numerical and experimental results of the works "Enhancement of sound in chirped sonic crystals" (see Appendix A.3), published in the journal Applied Physics Letters, and "Enhancement of sound by soft reflections in exponentially chirped crystals" (see Appendix A.4), published in a Special topic on Phononics in the journal AIP Advances. A novel mechanism of sound wave concentration in linear and exponential chirped sonic crystals is proposed and demonstrated. Special emphasis is made here in the description of the Coupled Mode Theory (CMT) used to interpret and estimate the wave concentration effect. We will concentrate here in the linear profile, discussing numerical and experimental results in frequency domain, and numerical simulations in time-domain, intended to fully interpret the reported wave concentration effect. 
Chirped crystals, also called graded or adiabatic tapered crystals, are a particular type of structures in which one of the parameters, such as the lattice constant or the filling fraction, gradually changes along the propagation direction. These type of structures were introduced in optics [Cassan et al., 2011] and acoustics [Kushwaha et al., 1998, Psarobas and Sigalas, 2002, Wu and Chen, 2011] for different purposes, such as opening wide full band gaps in tandem structures [Kushwaha et al., 1998], waveguiding of beams or also to control the spatial dispersion and focalization of beams in reflection [Cheng et al., 2014]. An interesting new phenomenon shown in chirped crystals is the smooth deflection of light beams from the straight trajectory as it propagates through the crystal, the so-called mirage effect [Centeno et al., 2006].

An important issue of wave propagation control and manipulation in periodic structures is the localization and concentration of the wave energy. Several mechanisms have been used to enhance wave energy at particular locations inside periodic structures. At a low frequency regime $(\lambda>>a$, being $a$ the lattice constant) for finite-size crystals, Fabry-Pérot resonances enhance the wave energy at particular frequencies $f_{n}$ inside the structure, which are related to its thickness, $L$. These frequencies are given by the expression $f_{n}=n c / 2 L$, where $n$ is an integer number and $c$ the speed of sound. For higher frequencies, where the wavelength and the lattice constant are of the same order $(\lambda \simeq a)$, the presence of point defects can produce localized enhanced modes at frequencies belonging to the band gap [Romero-García et al., 2010b, Romero-García et al., 2010a]. At these regimes, the wave can be enhanced for a set of narrow bands, but no broadband enhancement is achieved. An enhancement of the wave energy for a broader frequency range can be found via localized modes in random systems [Hu et al., 2008,Sainidou et al., 2005].

Another effect reported recently is the rainbow trapping effect, i.e., the dependence of the turning point position on the color (frequency) of radiation. It has been predicted for one-dimensional modulated chirped structures in photonics [Shen et al., 2011] and tapered optical and plasmonic waveguides [Stockman, 2004, Smolyaninova et al., 2010].

Rainbow trapping and wave enhancement are two different physical effects, while the former only appears for a certain set of frequencies, the latter may occur for a monofrequency radiation. However, both occur simultaneously in chirped crystals for a broadband incident radiation, as it will be demonstrated for sound waves in this chapter. 


\subsection{Coupled mode theory}

There are several reasons why CMT is worth to be applied to acoustic periodic media. Some of them are:

- CMT has been widely applied in photonics (Bragg fibers, phase gratings in holography, photonic waveguides,...). However, applications in acoustics are almost absent. The model itself is original.

- Our research group have previously applied CMT to acoustic problems in periodic media in linear regime: the lossy multilayers, and the chirped crystals. It is also beeing extended to study the nonlinear case of quadratic acoustic nonlinearity, although this case is not described in this thesis.

- Allows to obtain exact analytical solutions for the amplitudes of the stationary modes, and to analize their stability.

- It admits exact solitary wave solutions.

- and much more...

\subsubsection{CMT equations for linear propagation in fluids}

The propagation of sound waves in linear regime can be derivated from the linearized version of the continuity and Euler equations, completed by the equation of state. The linear acoustic wave equation is considered here as the starting point for the CMT discussed below

$$
\nabla^{2} p-\frac{1}{c_{0}^{2}} \frac{\partial^{2} p}{\partial t^{2}}=0
$$

where $p$ is the acoustic pressure and $c_{0}$ is the speed of sound.

\section{Field expansion}

A linear propagation problem is considered, where one wave with fequency $\omega$ propagates in a medium. In one-dimension, each wave has two possible directions of propagation (forward and backward). Assuming that the propagation 
occurs in a homogeneous medium, a general solution for a two-frequency field can given by

$$
p(x, t)=A e^{i k x-i \omega t}+B e^{-i k x-i \omega t}+\text { c.c. }
$$

where $A$ and $B$ are the (constant) amplitudes of the waves propagating to the right (forward) and to the left (backward). These waves may exist if the appropriate boundary conditions are given (excitation at the left or right boundaries).

\section{Modulation}

Consider now the propagation in a periodic structure. The parameters (density, velocity) change periodically in space along the wave propagation coordinate. We assume now that the acoustic wave velocity in the medium $c$ varies harmonically as:

$$
c=c_{0}+\delta c(x),
$$

where $c_{0}$ is the average velocity and $\delta c(x)$ can be expanded in Fourier series as:

$$
c=c_{0}+\Delta c \cos \left(k_{g} x\right)
$$

where $k_{g}=2 \pi / d$ is the grating wavenumber ( $d$ is the period of the modulation). Note that $k_{g}=2 k_{B}$, two times the band-gap value.

The perturbation of velocity scatters the right-going wave into a left-going wave, and viceversa. Under the coupled mode approximation, in the presence of a small modulation the fields take the same form as in the unperturbed medium, but may present small spatial and temporal variations in its amplitudes (which are induced by the modulation). The solution takes then the form:

$$
p(x, t)=A(x, t) e^{i k_{B} x-i \omega t}+B(x, t) e^{-i k_{B} x-i \omega t}+c . c .,
$$

where $A(x, t)$ and $B(x, t)$ are forward and backward propagation respectively. Note that we have chosen the reference wavenumber as the Bragg wavenumber. Then, we are considering solutions that describe modes around the band-gap, including modes in the propagation band (but close to the edge). This means that $A$ and $B$ are slowly varying functions (in space and time).

Substitution of the ansatz Eq. (3.5) and the sound velocity modulation Eq. (3.4), into the wave equation Eq. (3.1), results in a somehow complicated 
equation with many terms. However, it can be greatly simplified as soon as some approximations are considered (which are the assumptions of coupled mode theory), as:

- Weak velocity modulation $\delta c<<c_{0}$. Then we can expand the term $1 / c^{2}$ in series, and retain only the first order.

- Frequencies are close to a reference frequency (here, the bandgap frequency, $\left.k_{B}=k_{G} / 2=\pi / d\right)$, so $k=k_{B}+\Delta k$, with $\Delta k<<1$, and

- Slowly-Varying Envelope Approximation (SVEA), which is the assumption that the envelope of a forward traveling wave pulse varies slowly in space compared to a period or wavelength, where $\frac{\partial^{2} A_{1}}{\partial x^{2}} \ll k_{1} \frac{\partial A_{1}}{\partial x}$.

The coupled-mode equations for $A$ and $B$ are obtained after retaining only the first order phase-matched terms. Actually, projecting over the modes $\exp \pm i\left(\omega t-k_{B} x\right)$, neglecting off-resonance terms (those with an exponential different from zero) we get

$$
\left(\frac{\omega^{2}}{c_{0}^{2}}-k_{B}^{2}\right) A-\frac{\omega^{2} \Delta c}{c_{0}^{3}} B-2 i k_{B} \frac{\partial A}{\partial x}-\frac{2 i \omega}{c_{0}^{2}} \frac{\partial A}{\partial t}=0,
$$

which can be simplified to give

$$
\begin{aligned}
i\left(\frac{1}{c_{0}} \frac{\partial A}{\partial t}-\frac{\partial A}{\partial x}\right) & =\Delta k A+m B, \\
i\left(\frac{1}{c_{0}} \frac{\partial B}{\partial t}+\frac{\partial B}{\partial x}\right) & =\Delta k B+m A,
\end{aligned}
$$

where $\Delta k=k-k_{B}$, and $k=k(\omega)$, gives the detuning with respect to the Bragg resonance.

The group velocity is defined as $v=d w /\left.d k\right|_{\omega}$, and the modulation parameter $m$ is given by

$$
m=\frac{\Delta c}{c_{0}} \frac{k}{2}
$$

\subsubsection{Analytical solutions for linear and exponential profiles}

The CMT equations described previously are of application in one-dimensional problems, where the two media are characterized by its parameters (density, velocity), such as the multilayered structure depicted schematically in 
Fig. 3.1(a). However, taking into consideration a feasible realization of experimental measurements in acoustics, and more specifically, considering air as the background propagation medium, the huge impedance contrast between air and solid materials implies the absence of wave propagation within the latter i.e., the solid material is considered as a medium with infinite acoustic impedance, $Z=\rho c$. Hence, in order to allow wave propagation, the multilayered structure is substituted by a host medium with a periodical arrangement of rigid circular inclusions, as the one depicted in Fig. 3.1(b), which is indeed a two-dimensional medium. However, the CMT equations can be slightly modified proceeding to a dimension reduction, assuming that the contributions of the wave components propagating in transverse directions are negligible. This dimension reduction is possible because the first band gap in $\Gamma X$ direction essentially appears due to a resonant coupling between the forward and backward waves.
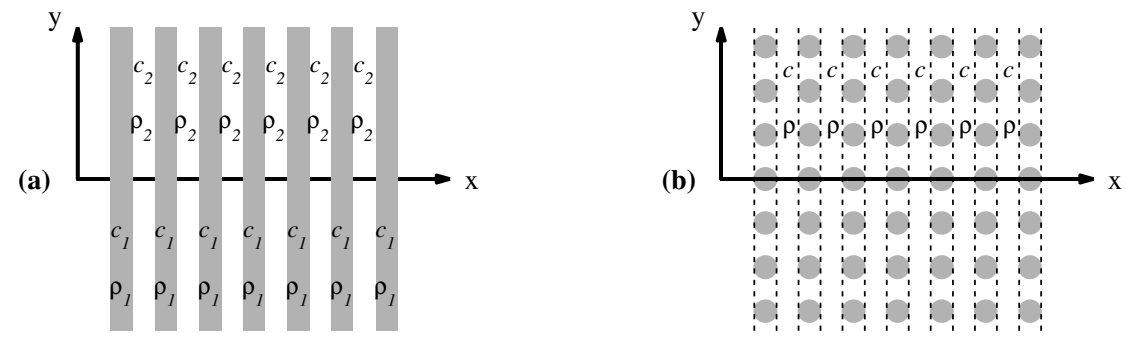

Figure 3.1: Schematic diagrams for (a) 1D Multilayered structure of a combination of two fluid media of finite impedances and (b) 2D Periodic arrangement of solid circular inclusions in a host medium of finite impedance.

\section{Linear chirped crystals}

Consider now a chirped sonic crystal with a linear variation of the lattice constant given by

$$
a(x)=a_{0}+\alpha\left(x-x_{0}\right),
$$

where $a_{0}$ is the lattice constant at the input plane of the crystal and $\alpha$ the adimensional chirp parameter. Assuming that the full pressure field consists of forward and backward propagating waves and taking a solution with an arbitrary reference wavenumber (not necessarily the bandgap), with a solution 
in the form

$$
p(x, t)=A(x, t) e^{i k x-i \omega t}+B(x, t) e^{-i k x-i \omega t}+c . c .
$$

we obtain the following equations:

$$
\begin{aligned}
i\left(\frac{1}{c_{0}} \frac{\partial A}{\partial t}-\frac{\partial A}{\partial x}\right) & =m B e^{i \Delta k x}, \\
i\left(\frac{1}{c_{0}} \frac{\partial B}{\partial t}+\frac{\partial B}{\partial x}\right) & =m A e^{-i \Delta k x} .
\end{aligned}
$$

Further consideration of stationary waves and substitution of modulation parameter $m$ by a factor $\sqrt{s}$, obtained numerically from the back scattered intensity by only one row of the structure, yields

$$
\begin{aligned}
& \frac{d A}{d x}=i \frac{\sqrt{s}}{a(x)} B e^{2 i \Delta q(x) x}, \\
& \frac{d B}{d x}=-i \frac{\sqrt{s}}{a(x)} A e^{-2 i \Delta q(x) x},
\end{aligned}
$$

where $\Delta q(x)=2 \pi / \lambda-\pi / a(x)$ is the detuning from the Bragg frequency. It is noticed that the detuning from the Bragg resonance $\Delta q(x)$ is a function of the longitudinal position $x$ for chirped crystals.

Equations (3.14) can be rewritten in canonical form as

$$
\frac{d^{2} A}{d X^{2}}=i \epsilon(x) \frac{d A}{d X}+A,
$$

where the space scaling $d X=d x \sqrt{s} / a(x)$ was chosen to make the normalized coupling coefficient unity, and $\epsilon(x)=2 d(X \Delta q(X) / d X$ is the normalized detuning from the Bragg frequency.

In general (for arbitrary chirp) Eq. (3.16) cannot be solved analytically. However, in a simple case when the normalized detuning varies linearly around zero $\epsilon(X)=\epsilon_{1}\left(X-X_{0}\right)$, Eq. (3.16) has an analytical solution in the form

$$
A(X)=c_{1} H_{i / \epsilon_{1}}\left(X \sqrt{i \epsilon_{1} / 2}\right),
$$

where $H_{n}$ is the Hermite polynomial of imaginary order. The counter-propagating field obeys a similar expression. The integration constant $c_{1}=H_{i / \epsilon_{1}}\left(X_{F} \sqrt{i \epsilon_{1} / 2}\right)$ is determined by the boundary conditions, by imposing that the amplitude of 

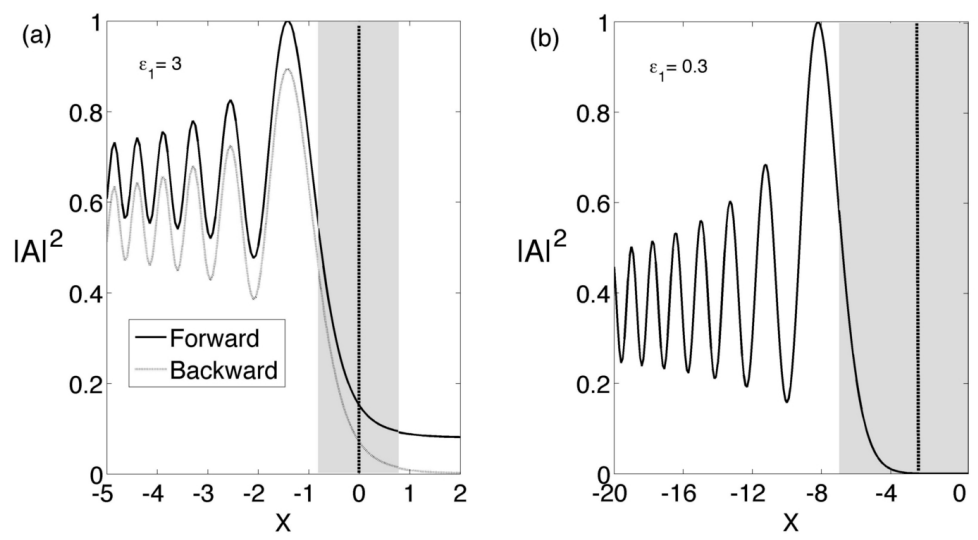

Figure 3.2: Solutions of Eq. (3.17), i.e. the intensity of the forward (continuous line) and backward (dashed line) field along the chirped structure. (a) (with $\epsilon=3$ ) corresponds to experimental configuration, (b) (with $\epsilon=0.3$ ) is shown for comparison, to illustrate soft reflections for a substantially smaller chirp. The vertical dashed lines indicate the center of the bandgap, and the shaded areas the bandgap itself.

the forward wave at the front face $X=X_{F}$ equals unity. $\epsilon_{1}=d \epsilon(X) /\left.d X\right|_{X=X_{F}}$ or, in terms of initial variables, $\epsilon_{1}=4 \pi \alpha / s$, which estimated for experimental parameters results $\epsilon_{1}=3$.

In Fig. 3.2 we present the amplitude of the acoustic intensity of the forward and backward waves for linearly chirped crystals as follows from Eq. (3.17). The acoustic field is nearly exponential in the bandgap, and oscillatory in front of it. The oscillations, with the period and amplitude increasing as the wave approaches the band-gap, are large-scale oscillations, which originate from the energy exchange between the forward and backward waves. These large-scale oscillations correspond to oscillations of the envelope of the Bloch modes and are not due to conditions imposed at the entrance of the sonic crystal, e.g. some possible impedance mismatch.

The controlled field enhancement effect is clearly visible again in Fig. 3.2. From the analytical estimations in Eqs. (3.14)-(3.17) and from Fig. 3.2 it follows that for maximal field enhancement of the wave intensity, the chirp must be as small as possible. For $\epsilon_{1}=0.3$ the maximum field enhancement could be around 6 times (in terms of intensities) if one compares the maximal and minimal values of the plot in Fig. 3.2(b). 


\section{Exponential chirped crystals}

Consider now a chirped crystal with an exponential variation of the lattice constant defined as a function of the scaled spacing as follows

$$
a(X)=e^{\alpha X}-1 .
$$

In this case an analytical solution also exists, given by

$$
A(X)=e^{-\alpha X} L_{n}^{s}\left(\frac{i e^{\alpha X}}{\alpha}\right),
$$

where $L_{n}^{s}$ is a generalized Laguerre polynomial, with the indexes defined as $n=(-1)^{5 / 6} / \alpha$ and $s=\sqrt{3} / \alpha$.

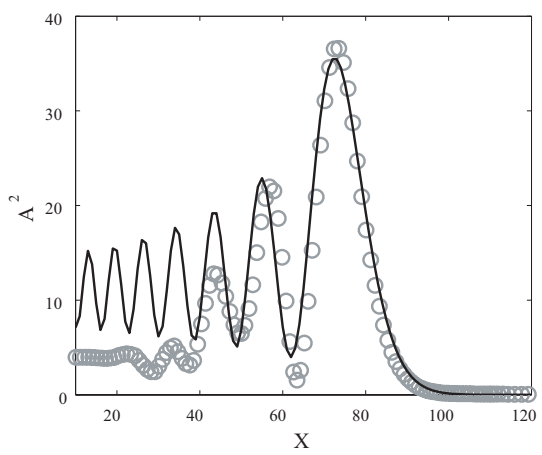

Figure 3.3: (Color online) Continuous line represents the amplitude profile in the exponentially chirped crystal, as given by the analytical solution of Eq. (3.19) calculated using $\alpha=-0.05$. Green dots represents the envelope for the experimental case.

Fig. 3.3 illustrates the profile of the squared amplitude of the wave (normalized intensity) along the chirped structure. Continuous black line represents the results from Eq. (3.19) for a chirp parameter $\alpha=-0.05$, and green open dots represents the envelope of the experimental data for the case of $2700 \mathrm{~Hz}$ shown in "Enhancement of sound by soft reflections in exponentially chirped crystals". A significant enhancement of the wave amplitude appears at the end of the path of the wave (bright plane), just before the turning plane (local band gap). It is noticed that due to the range of validity of the model, is in the range of frequencies near the band gap in which the theory agrees well with the experiments, as expected. The region in which these 
oscillations become important is much smaller than in the case of linear chirp and the amplitude is more localized (see Fig. 5 (b) in "Enhancement of sound in chirped sonic crystals").

\subsection{Wave dispersion in chirped structures. Soft re- flection}

Waves propagating in periodic structures at frequencies at and close to the band gap have a very small or zero group velocity, $v=\partial w / \partial k$, as it can be extracted from the analysis of the nearly flat segments of the dispersion curves $w(k)$. For frequencies belonging to the band gap, where waves are not allowed to propagate, reflection of incoming waves is similar to the reflection of waves impinging a rigid wall. In contrast, wave reflection from a chirped structure is different. A wave, whose frequency is fixed and above the first band gap, entering into a chirped sonic crystal, is gradually slowed down as it penetrates, as the local band gaps are approaching the wave frequency in the course of propagation. At a particular depth corresponding to the band edge, the wave stops, turns around, and starts propagating back, experiencing a "soft" or "smooth" reflection. It is assumed that for a slow variation of the lattice constant within the structure, each plane can be characterized by a local dispersion relation, i.e., the dispersion relation of an infinitely extended periodic crystal for parameters (lattice constant, filling fraction) corresponding to a particular depth of the chirped crystal.

Figure 3.4 illustrates this effect for three different frequencies. The evolution of the band gaps along a chirped sonic crystal with a linear profile of the lattice constant $a=a(x)$ is shown in red, as well as the local dispersion relation at the entrance (first plane) and exit (last plane) of the system. Insets in the bottom of Fig. 3.4 represent the spatial distribution of the rigid scatterers inside the chirped structure as well as the acoustic field for 2450 $\mathrm{Hz}, 2650 \mathrm{~Hz}$ and $2825 \mathrm{~Hz}$. It is noted that for each of these highlighted frequencies the acoustic field is enhanced at a particular plane, i.e., the brilliant plane, due to the coupling between forward and backward waves, and quickly and strongly attenuated behind. Besides, the position of the maximum of the acoustic energy inside the structure is shifted as the frequency increases (rainbow effect), in correspondence with the aforementioned assumption of local dispersion relation. 


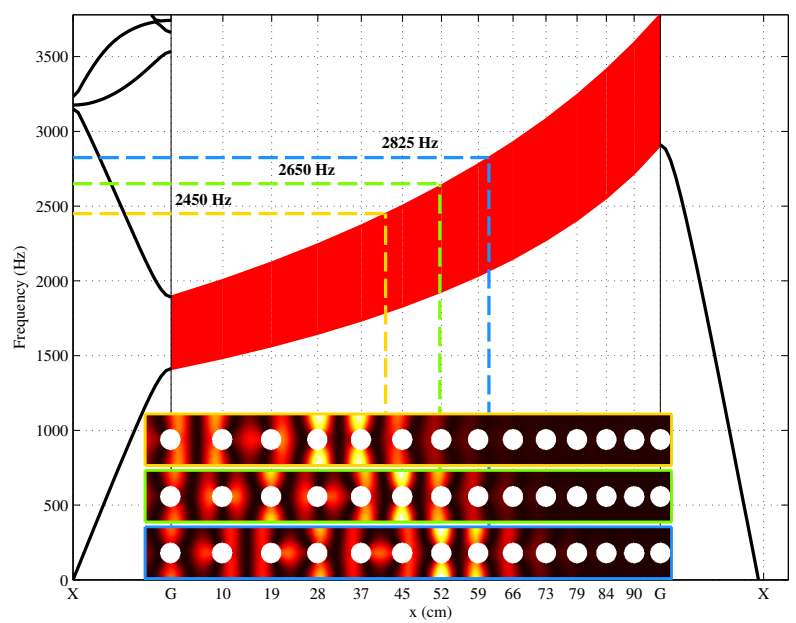

Figure 3.4: Local band gaps in a linear chirped structure. Left(right) panel shows the band structure at the entrance(exit) of the system calculated using the finite element method. Middle panel show the evolution of the upper and lower band edges of the band gap within the discrete structure. Insets in the bottom show the spatial distribution of the scatterers in the system as well as the acoustic field for three different frequencies, $2450 \mathrm{~Hz}, 2650 \mathrm{~Hz}$ and $2825 \mathrm{~Hz}$.

\subsubsection{Harmonic analysis. Spatial enhancement of the acoustic field}

A linear chirped sonic crystal was designed to numerically and experimentally validate the analytical results obtained solving Eqs. (3.14)-(3.17). The structure consists of a two-dimensional crystal with rectangular local symmetry made of acoustically rigid aluminum cylinders of radius $r=2 \mathrm{~cm}$, embedded in air. The spatial period is constant in the direction transverse to propagation direction $y, a_{y}=10 \mathrm{~cm}$, while a linear variation of the lattice constant is defined in the longitudinal direction $x$. The adimensional chirp parameter is $\alpha=\left(a_{j}-a_{j+1} / a_{j}\right)$, where $a_{j}$ is the longitudinal lattice constant at the $j$ th layer. For the structure considered in this section the initial period is $a_{0}=10 \mathrm{~cm}$, the final period $a_{13}=4.8 \mathrm{~cm}$, and the chirp parameter $\alpha=0.055$. Hence, the structure consists of 14 scatterers in the longitudinal direction $x$. 6 scatterers are considered in the transverse to propagation direction $y$.

Sound pressure amplitude, $|p|$, is evaluated for a longitudinal cut along $x$ axis between two rows of scatterers for a wide frequency range. The recorded amplitude is normalized with respect to the amplitude of the incident field, 
$\left|p_{0}\right|$. Since the incident amplitude is normalized to unity, the total intensity resulting from the reflection of a purely periodic structure in the frequency range of the band gap (or almost acoustically equivalent, a rigid wall), can be increased a maximum of 4 times, as the interference is formed from forward and fully reflected backward waves.
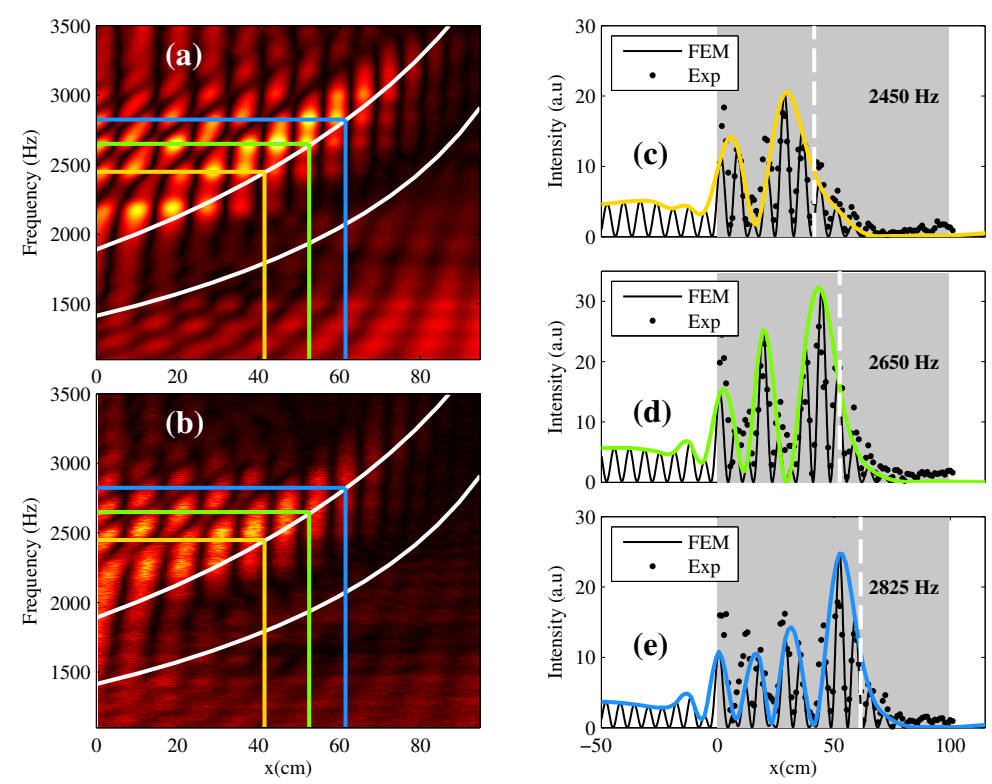

Figure 3.5: (a) Numerical simulations and (b) experimental results of the acoustic field, $|p|$, inside the linear chirped sonic crystal. White continuous lines define the limits of the upper and lower band edges of the spatially varying local band gaps. Coloured lines illustrate the frequencies shown in (c), (d), (e) as well as the corresponding positions of the turning planes inside the crystal. (c), (d), (e) represent the normalized acoustic intensity, $I / I_{0}=\left|p^{2}\right| /\left|p_{0}\right|^{2}$ for a longitudinal cut at frequencies $2475 \mathrm{~Hz}, 2600 \mathrm{~Hz}$ and $2700 \mathrm{~Hz}$, respectively. Grey shaded rectangles denote the area covered by the linear chirped crystal.

Figure. 3.5(a)-(b) represents two-dimensional frequency-space maps showing the normalized acoustic field, $|p| /\left|p_{0}\right|$, inside the structure for numerical calculations and experimental measurements, respectively. White continuous lines represent the lower and upper edges of the band gap along the chirped structure. From the analysis of these maps several features are noted. The enhancement is observed at the brilliant planes, located just before the upper band edges. Moreover, the position of these planes is shifted to deeper positions inside the structure as the frequency is increased, as expected. Hence, the total intensity is enhanced for a certain range of frequencies, in opposition 
to the case of perfectly periodic crystals in which only discrete frequencies can be enhanced by Fabry-Pérot resonances. The finite-size of the structure in transversal direction (6 rows of scatterers) prompts the appearance of edge effects which are responsible for the reduction of the enhancement for frequencies above $2700 \mathrm{~Hz}$, to be discussed below. However, a very good agreement is found between simulations and experiments. Three particular frequencies illustrate quantitatively the enhancement of the normalized total intensity, $I / I_{0}$, as shown in Fig. 3.5 (c, d, e), where the axial distributions obtained experimentally (dots) and numerically (black lines) are shown. Small-scale fringes are observed in both cases, corresponding to the local Bloch mode, as well as large-scale oscillations or envelope (continuous coloured lines) of the Bloch mode. The recorded total intensity is up to 20-30 times higher than the intensity of the incident wave.

(a)

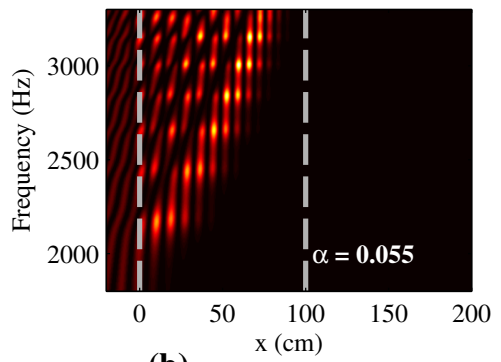

(b)

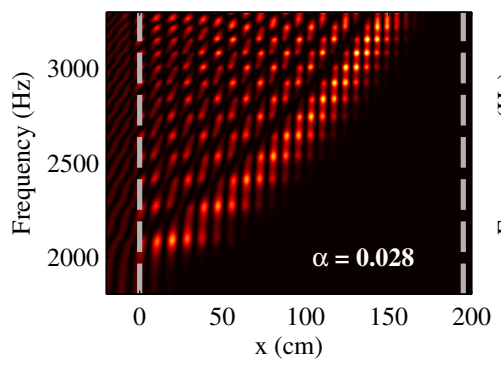

(c)

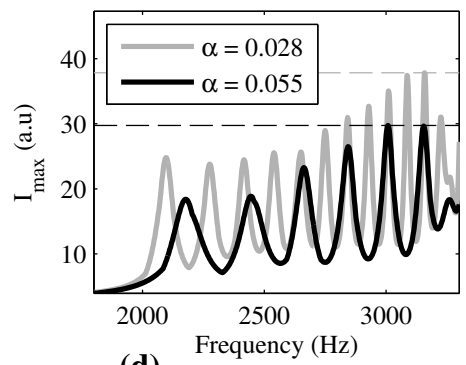

(d)

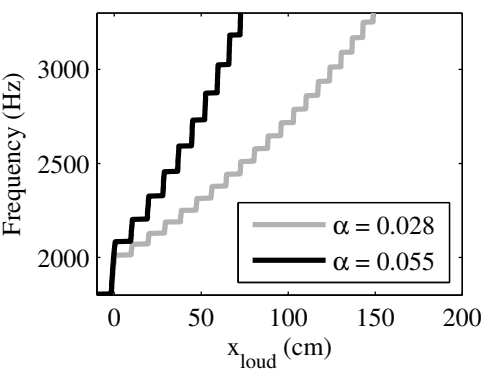

Figure 3.6: Numerical simulations showing the normalized pressure field, $|p| /\left|p_{0}\right|$, in frequency-space maps for a longitudinal cut for two linear chirped structures with gradient (a) $\alpha=0.055$, (b) $\alpha=0.028$. Dashed grey lines indicate the area covered by the structures. (c) Maximum value of the normalized total intensity, $I_{\max } / I_{0}$, depending on frequency. (d) Position of the maximum value of concentration of acoustic intensity (rainbow effect).

The influence of the chirp parameter, $\alpha$, is analyzed via numerical calculations and compared to the results shown in Fig. 3.5. An additional linear 
chirped structure is designed for that purpose. Design parameters for this additional chirped structure are the same as the previous one but $\alpha$, i.e., radius $r=2 \mathrm{~cm}$, transversal period $a_{y}=10 \mathrm{~cm}$, initial period, $a_{0}=10 \mathrm{~cm}$, final period $a_{13}=4.8 \mathrm{~cm}$. The new value of the chirp parameter is $\alpha=0.028$, resulting in a structure having a total length that almost doubles the length of the previous one. To overcome the limitations found due to the finite-size of the structure in the transversal direction, Bloch-Floquet periodic boundary conditions are imposed in numerical calculations in the upper and lower boundaries. Thus, both structures are infinite in transversal direction.

Results from the comparison between them are shown Fig. 3.6. Frequencyspace maps representing the normalized pressure acoustic field in Fig. 3.6(a),(b) indicate that the position of the maximum value of concentration of acoustic energy is found at deeper positions inside the longest structure. For the sake of simplicity, the position of these maximum values depending on frequency is represented in Fig. 3.6(d). Enhancement of the total intensity is higher for chirped crystals with smaller $\alpha$. In agreement with Fig. 5 in "Enhancement of sound in chirped sonic crystals", the maximum field enhancement is found for the longest chirped structure. Figure. 3.6(b), where the value of the normalized total intensity at the position indicated in Fig. 3.6(d) depending on frequency is represented, validates the previous statement. It is noted that the resonant peaks are due to Fabry-Pérot resonances where the equivalent length is that of the brilliant plane at each frequency.

\subsubsection{Time-domain analysis.}

While harmonic analysis is suitable to understand several characteristics of the soft reflection of waves inside chirped sonic crystals, wave propagation inside chirped sonic crystals in time domain adds a different point of view of the problem and facilitates a fully understanding of this effect. The effects on the character of the time spreading of a pulse penetrating on a chirped crystal are analyzed here. Numerical simulations are carried out using the finite element method in time-domain for the same linear chirped crystal used previously, with gradient $\alpha=0.028$ and the same frequencies, 2425, 2650 and 2850

$\mathrm{Hz}$. A gaussian pulse centered at these frequencies with a fixed bandwidth $\Delta f=100 \mathrm{~Hz}$ is emitted from the left boundary of the domain propagating in $+x$ direction.

Figure. 3.7 illustrates the time-space scenario for $f=2650 \mathrm{~Hz}$ by com- 
paring the reflection of a gaussian pulse from a periodic structure, designed to have a band gap at this frequency, with the soft reflecion ocurring in a chirped crystal. The lattice constant of the periodic structure is $a=6.72 \mathrm{~cm}$, the same as the $15^{\text {th }}$ plane of the chirped crystal, and it is placed at the same point, $x=123.88 \mathrm{~cm}$, which corresponds to the turning plane of the chirped structure at the working frequency, in order to facilitate the comparison between both cases.
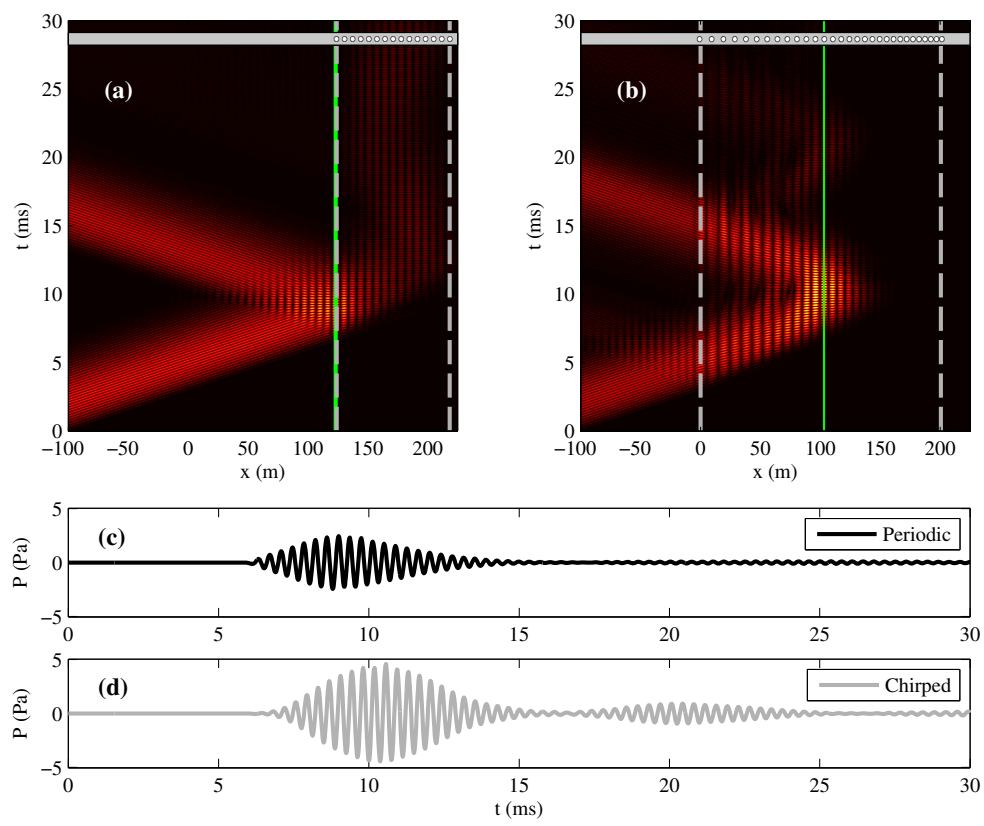

Figure 3.7: Time spreading of the input signal in for $f=2650 \mathrm{~Hz}$. Top insets represent the time-space scenario for a longitudinal cut along the propagation direction $x$, for (a) a periodic structure with fixed lattice constant $a=6.72 \mathrm{~cm}$, placed in the range $x=$ $[123.8,217.9] \mathrm{cm}$, as a reference. (b) linear chirped structure with a gradient $\alpha=0.028$, placed in the range $x=[0,200] \mathrm{cm}$. Dashed grey lines indicate the area covered by the structures. The spatial distribution of the scatterers is included in top of insets (a), (b). Recorded time signals for (c) periodic structure and (d) linear chirped structure at the positions indicated by green solid lines, where the maximum value of pressure is found.

The relation between $x$ and $t$ is a straight line and its slope is given by the sound velocity in air. The gaussian pulse is almost totally reflected by the periodic structure and only a very small part of it penetrates having an exponential decay (evanescent wave). Reflection inside the chirped crystal is different, the incident wave is gradually slowing down and soft reflected 
at the turning plane, resulting in a bright plane due to the reduction of the group velocity, as shown in previous sections. Afterwards, the pulse travels back out of the structure. It is also observed that the impedance constrast between the homogeneous medium and the chirped crystal causes the wave to be reflected back and forth between the entrance and the turning plane of the chirped crystal until the amplitude is vanished. As it will be discussed later, the estimation of the delay in time between these contributions allow to evaluate and compare the sound velocity within the chirped crystal to that obtained by the analysis of the group velocity extracted from the dispersion curves, validating the assumption of a local dispersion relation. Figure. 3.8 is intended to illustrate the validity of the aforementioned assumption.

(a)

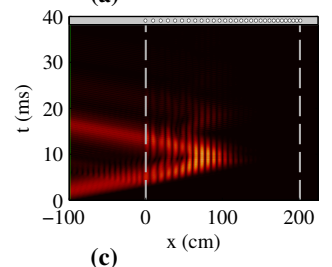

(c)

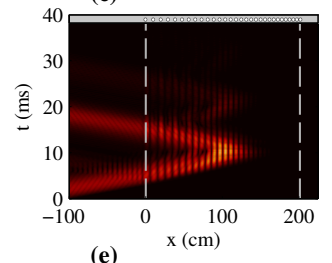

(e)

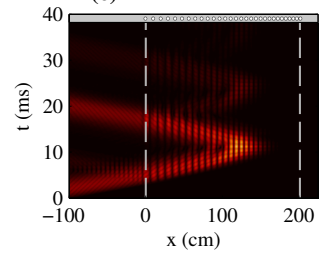

(b)

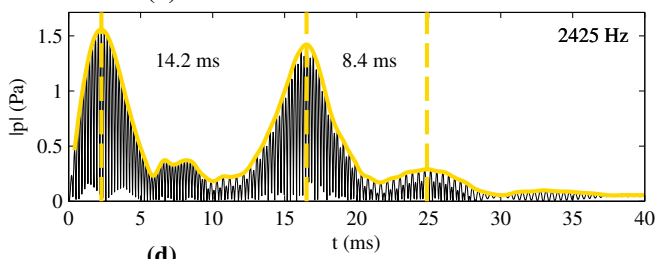

(d)
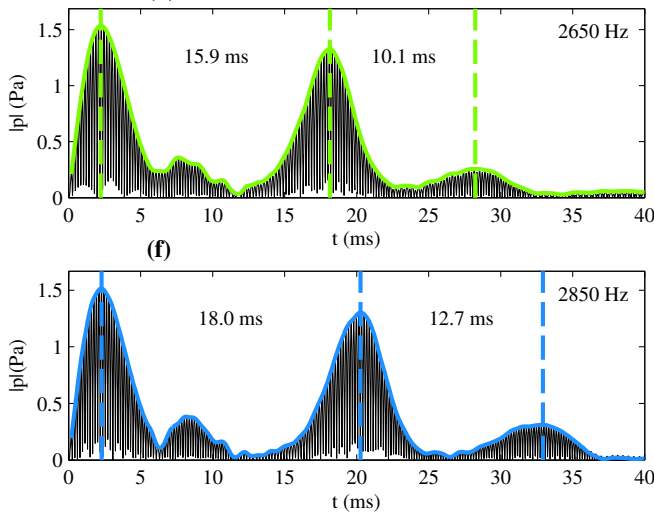

Figure 3.8: Time-space maps showing the time signals recorded for a longitudinal cut along the propagation direction $x$, for (a) $2425 \mathrm{~Hz}$, (c) $2650 \mathrm{~Hz}$ and (e) $2850 \mathrm{~Hz}$. (b), (d) and (f) represent the time signals at $x=-100 \mathrm{~cm}$. Amplitude is given by the normalized absolute value of the pressure $|p| /\left|p_{0}\right|$.

Time-space maps for 2425, 2650 and $2850 \mathrm{~Hz}$ are illustrated in Fig. 3.8(a), (c), (e), where grey dashed lines indicate the area covered by the chirped structure. Time profiles evaluated at $x=-100 \mathrm{~cm}$ are represented in Fig. 3.8(b), (d) and (f), where different contributions are clearly observed. A closer look to any of these time signals, i.e., Fig. 3.8(d), allows to identify the incident 
pulse $(0-5 \mathrm{~ms})$, the reflected wave in the input plane of the crystal due to a certain impedance contrast between the homogeneous medium and the chirped structure $(7-12 \mathrm{~ms})$, the soft reflected wave in the bright plane inside the chirped structure $(12-20 \mathrm{~ms})$, and finally a secondary soft reflected contribution, corresponding to some part of the first soft reflected wave scattered back to the chirped structure $(22-30 \mathrm{~ms})$. From the time delay between different contributions, the slowing down of the wave can be quantified and compared to the results extracted from the slopes of the band structures at each plane in the chirped structure $\left(v_{g}=\partial w / \partial k\right)$. Table 3.1 shows the group velocity for each plane of the structure for the three frequencies analyzed in this Section. Taking into account these values as well the sound velocity and propagation distance in air, the delay between different contributions (highlighted by coloured dashed lines in Figs. 3.8(b), (d) and (f)) can be calculated using the following expressions

$$
\begin{gathered}
t_{1, f}^{\text {delay }}=2\left(\frac{x_{a i r}}{c_{a i r}}+\int \frac{d x}{v_{f}(x)}\right)=2\left(\frac{x_{a i r}}{c_{a i r}}+\sum_{i=1}^{N_{f}} \frac{a_{i}}{v_{f, i}}\right) . \\
t_{2, f}^{\text {delay }}:=2 \int \frac{d x}{v_{f}(x)}=2 \sum_{i=1}^{N_{f}} \frac{a_{i}}{v_{f, i}},
\end{gathered}
$$

where the integral has been replaced by a sum considering that the velocity is constant and equal to the value deduced from the band structures that corresponds to the lattice constant, $a_{i}$, for every crystal plane till reaching the corresponding turning plane at each frequency. These values are included in Table 3.1 and compared to the ones obtained by time-domain simulations, named $t_{1, \text { sim }}, t_{2, \text { sim }}$, for the time delay between incident and first soft reflected, and first and second soft reflected pulse, respectively. The agreement between these estimations validates the local dispersion relation assumption and is consistent with the results obtained for the exponential chirped structure presented in the work "Enhancement of sound by soft reflections in exponentially chirped crystals".

This chapter has been devoted to the study of linear and exponential chirped sonic crystals. A mechanism for sound field enhancement has been predicted and demonstrated numerically and experimentally. The acoustic wave energy can be concentrated at a particular depth of the crystal depending on the frequency and on the parameters of the structure. At these bright planes, a substantial increase of the energy is observed for frequencies around 


\begin{tabular}{|c|c|c|c|}
\hline \hline Lattice constant & \multicolumn{3}{|c|}{$v_{g}\left(\mathrm{~ms}^{-1}\right)$} \\
\hline \hline$a(\mathrm{~cm})$ & $2425 \mathrm{~Hz}$ & $2650 \mathrm{~Hz}$ & $2850 \mathrm{~Hz}$ \\
\hline 10,00 & 297.64 & 295.62 & 285.04 \\
\hline 9,72 & 293.56 & 293.69 & 284.10 \\
\hline 9,45 & 290.51 & 290.76 & 281.12 \\
\hline 9.18 & 284.46 & 285.46 & 277.55 \\
\hline 8.92 & 275.90 & 281.43 & 273.85 \\
\hline 8.67 & 263.29 & 276.55 & 270.08 \\
\hline 8.43 & 243.88 & 270.37 & 266.14 \\
\hline 8.20 & 212.88 & 262.00 & 256.04 \\
\hline 7.97 & 163.45 & 255.83 & 252.20 \\
\hline 7.74 & 48.51 & 244.17 & 247.40 \\
\hline 7.52 & 0 & 226.14 & 240.71 \\
\hline 7.32 & 0 & 197.21 & 230.52 \\
\hline 7.11 & 0 & 85.18 & 222.52 \\
\hline 6.91 & 0 & 44.71 & 207.22 \\
\hline 6,72 & 0 & 0 & 181.54 \\
\hline 6,53 & 0 & 0 & 42.58 \\
\hline 6.35 & 0 & 0 & 0 \\
\hline \hline$t_{1, f}^{\text {delay }}(\mathrm{ms})$ & 15.4 & 18.4 & 18.8 \\
\hline$t_{1, \text { sim }}(\mathrm{ms})$ & 14.2 & 15.9 & 18.0 \\
\hline$t_{2, f}^{\text {delay }}(\mathrm{ms})$ & 9.6 & 12.6 & 12.9 \\
\hline$t_{2, \text { sim }}(\mathrm{ms})$ & 8.4 & 10.1 & 12.7 \\
\hline \hline & & & \\
\hline \hline
\end{tabular}

Table 3.1: Values of the group velocity deduced from the band structures calculated using FE.

the first gap along the $x$-direction of the structure. The CMT employed to predict the effect has been desccribed, as well as numerical simulations and experimental results. Further conclusions wil be given in Chapter 6 .

\section{References}

Cassan, E., Caer, C. K.-V. D., Marris-Morini, D., and Vivien, L. (2011). Short-wavelength light propagation in graded photonic crystals. J. Lightwave Tech., 29:1937-1943.

Centeno, E., Cassagne, D., and Albert, J.-P. (2006). Mirage and superbending effect in two-dimensional graded photonic crystals. Phys. Rev. B, 73:235119.

Cheng, Y., Kicas, S., Trull, J., Peckus, M., Cojocaru, C., Vilaseca, R., Drazdys, R., and Staliunas, K. (2014). Flat focusing mirror. Sci. Rep., 4:6326.

http://functions.wolfram.com/HypergeometricFunctions/LaguerreL 3General/. 
Hu, H., Strybulevych, A., Page, J. H., Skipetrov, S. E., and van Tiggelen, B. A. (2008). Localization of ultrasound in a three-dimensional elastic network. Nature Physics, 4:945.

Kushwaha, M., Djafari-Rouhani, B., L-Dobrynski, and Vasseur, J. (1998). Sonic stopbands for cubic arrays of rigid cylinders in air. Eur. Phys. J. B, 3:155-161.

Psarobas, I. E. and Sigalas, M. M. (2002). Elastic band gaps in a fcc lattice of mercury spheres in aluminum. Phys. Rev. B, 66:052302.

Romero-García, V., Sánchez-Pérez, J., and Garcia-Raffi, L. (2010a). Evanescent modes in sonic crystals: Complex dispersion relation and supercell approximation. J. Appl. Phys., 108:044907.

Romero-García, V., Sánchez-Pérez, J., neira Ibáñez, S. C., and Garcia-Raffi, L. (2010b). Evidences of evanescent bloch waves in phononic crystals. Appl. Phys. Lett., 96:124102.

Sainidou, R., Stefanou, N., and Modinos, A. (2005). Widening of phononic transmission gaps via anderson localization. Phys. Rev. Lett., 94:205503.

Shen, Y., Fu, J., and Yu, G. (2011). Rainbow trapping in one-dimensional chirped photonic crystals composed of alternating dielectric slabs. Phys. Lett. A, 375:3801-3803.

Smolyaninova, V. N., Smolyaninov, I. I., Kildishev, A. V., and Shalaev, V. M. (2010). Experimental observation of the trapped rainbow. Appl. Phys. Lett., 96:211121.

Stockman, M. (2004). Nanofocusing of optical energy in tapered plasmonicwaveguides. Phys. Rev. Lett., 93:137404.

$\mathrm{Wu}$, L. and Chen, L. (2011). An acoustic bending waveguide designed by graded sonic crystals. J. Appl. Phys., 110:114507. 



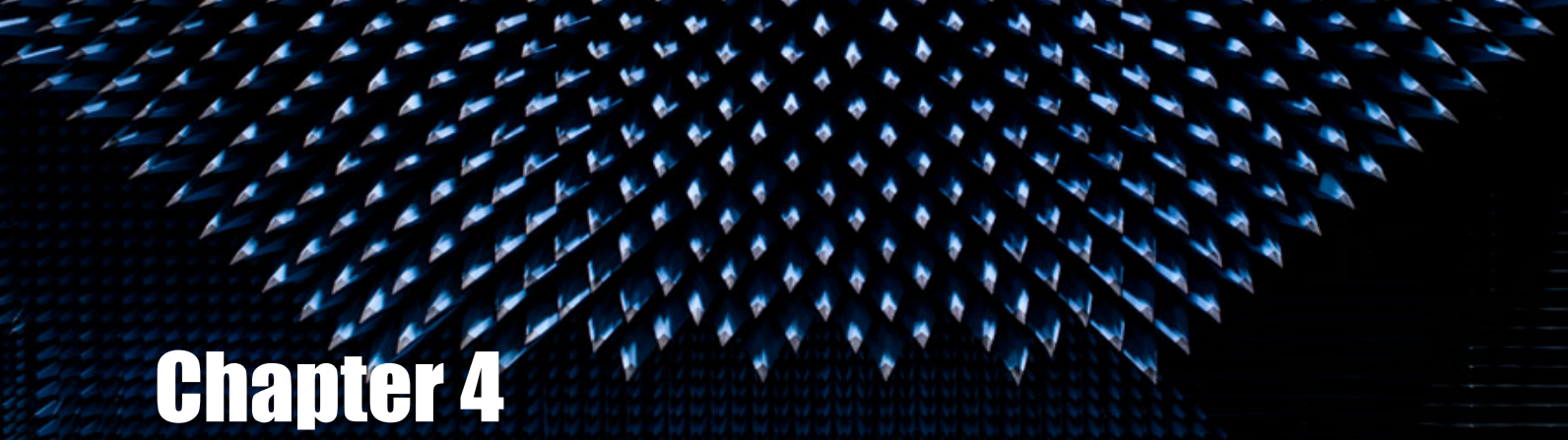

\section{Absorption in Periodic Structures}

This chapter is devoted to the study of absorption phenomena in periodic structures. Section 4.1 is devoted to the work Enhanced transmission band in periodic structures with loss modulation, published in the journal Applied Physics Letters (see Appendix A.5). In this work the propagation of in-plane sound waves in a periodic array of absorbing layers is studied, reporting an enhancement of wave transmission around the Bragg frequencies. Section 4.2 is devoted to the work Extraordinary absorption of sound in porous lamellacrystals published in the journal Scientific Reports (see Appendix A.6). It consists in the design of a structured material supporting complete and broadband absorption of out-of-plane waves that penetrate into a periodic array of porous layers. This work is the result of a colaboration of our research group with people from the Technical University of Denmark. A detailed description is given on the experimental measurements, carried out in our research facilities. 
In the previous chapters the propagation of acoustic waves in periodic media has been considered for conversative systems, where losses are neglected in the derivation of the inhomogeneous wave equation. This assumption is valid in situations where dissipation is insignificant, allowing the study of different interesting phenomena in periodic media, such as focalization, reflection of waves at the band gaps, scattering or even localization inside crystals. However, dissipation is an inherent property of all forms of matter and its effects in periodic media are of great interest, as waves cannot be absorbed unless dissipation is considered.

The fact that light and sound waves behave in the same manner in linear media, obeying similar wave equations, has inspired a number of analogies between both fields. However, the motivation for the study of losses in acoustics and optics may be different in some cases. In optics, several recent works have been devoted to minimize losses. Some of them investigate how the absorption is reduced in a multilayered magneto-photonic crystal [Figotin and Vitebskiy, 2008,Figotin and Vitebskiy, 2010], while others report an enhanced transmission through a stack of dielectric layers having contrast only in attenuation Refs. [Erhokhin et al., 2008, Kumar et al., 2012]. Extensions to two-dimensional modulation of losses have shown to provide nontrivial light beam propagation effects, analogous to flat photonic crystal lensing reported in conservative systems [Staliunas et al., 2009, Kumar et al., 2013]. In acoustics, the situation is different, since achieving maximum absorption is often the goal. The effect of viscoelastic losses on phononic crystals has been discussed in terms of the modification of dispersion relations [Psarobas, 2001, Lee et al., 2010,Laude et al., 2013,Hwan Oh et al., 2013]. Damping of elastic waves in solid phononic crystals has also been discussed [Hussein, 2009, Andreassen and Jensen, 2013]. However, the behaviour of lossy periodic media for waves near Bragg resonances is much less known than in the long-wavelength limit [Tournat et al., 2004]. Motivated by the aforementioned studies in optics [Erhokhin et al., 2008, Kumar et al., 2012], section 4.1 is devoted to the study of wave propagation within a layered material with periodically distributed losses, showing how the periodicity of the absorbing media can modify the global absorption of the system as well as its reflection and transmission properties.

In the context of enhancing the absorption for acoustic waves in audible regime, the acoustic equivalent to an ideal black body in optics would be something similar to a "deaf" body, which is an object absorbing sound coming from all directions at any given frequency. Absorption of sound waves is gov- 
erned by the effects of viscosity and thermal conduction in fluids. In order to deal with these dissipative processes at the macrospopic level the mass density $\rho$ and the bulk modulus $K$ are to be considered as complex quantities, leading to a finite penetration length into the dissipative medium. While the concept of a deaf body remains to be the ideal case, various composites and artificial structures have been designed in the attempt of pursuing this ultimate goal. In this sense, a distinction between resonance-based and broadband systems is made. Locally resonating materials have been fabricated in the form of mass-loaded thin membranes, gas-bubble arrays and elastic beams, featuring sharp and narrow absorption peaks [Mei et al., 2012,Leroy et al., 2009b,Leroy et al., 2009a, Thomas, 2009, Romero-García et al., 2011]. Broadband absorption, on the other hand, has been demonstrated for low frequencies by lattices of perforated shells [García-Chocano et al., 2012].

Periodic penetrable structures, generally speaking, have been fabricated with many facets for different kinds of waves. Electromagnetic (EM) structured materials, in that regard, such as gratings with finite conductance, convex grooves or the moth eyes, comprise anti-reflective systems with a broad spectral response [Botten et al., 1981, McPhedran et al., 1982, Kravets et al., 2009, Søndergaard et al., 2012, Clapham and Hutley, 1973]. However, to sustain a spectrally and angularly rich performance with complete absorption and little material use remains a challenge to be solved.

The system analyzed in section 4.2 is motivated by recent EM experiments where a forest of vertically aligned single-walled carbon nanotubes showed extremely low reflectance [Garcia-Vidal et al., 1997, Yang et al., 2008, GarciaVidal, 2008, Mizuno et al., 2009, Lidorikis and Ferrari, 2009]. The mechanism consists in the attempt of matching the material index to free-space to prevent back-reflected waves but at the same time providing sufficient material losses to guarantee intensity attenuation. The system, a low-density porous lamella array, in analogy to its electromagnetic counterpart made of nanotube arrays, behaves most closely like a true deaf body. Within this framework, we show how these constructed crystals become more absorptive when less material is chosen. 


\subsection{Enhanced transmission band in periodic struc- tures with loss modulation}

\subsubsection{Dissipative coupled mode theory}

The CMT described in Chapter. 3 in the context of acoustic periodic media is of application in conversative systems. However, this model constitutes a particular case of a more general problem in which dissipation is involved. Our interest now is to study a multilayered medium formed by a finite number of lossy paralel and equidistant layers and calculate the influence of losses in the transmission and reflection spectrum. Hence, the model is now formulated including the effect of the dissipation. Consider the aforementioned multilayered lossy medium irradiated by an incident plane wave. The total field is described in terms of forward and backward propagating waves

$$
P=A(x) e^{i k_{B} x-i \omega t}+B(x) e^{-i k_{B} x-i \omega t}+c . c
$$

where $A(x), B(x)$ are the normalized forward and backward waves amplitudes, such that their absolute square is proportional to the energy flux in the corresponding direction. $k_{B}=\pi / a$ is the Bragg wavenumber (the edge of Brillouin zone), $a$ the lattice constant and $\omega$ is the angular frequency. Considering frequencies near a Bragg resonance, the dynamics of the coupled forward and backward waves can be described by the dissipative coupled-mode equations

$$
\begin{aligned}
\frac{d A}{d x} & =i \Delta k A+m B+\gamma A, \\
-\frac{d B}{d x} & =i \Delta k B+m A+\gamma B,
\end{aligned}
$$

where $\Delta k=k-k_{B}=2 \pi / \lambda-\pi / a$ is the detuning from the Bragg wavenumber, $m=m_{R e}+i m_{I m}$ is the coupling between forward and backward waves and $\gamma$ is the gain coefficient, which is always negative in acoustic lossy media $(\gamma<0$, since there are no gain acoustic materials, in contrast to an optical gain medium, etc.). The coupling coefficient, $m$, is generally complex; real for conservative media (wave velocity modulation for the acoustic case, see Eqs. (3.3), (3.4) in Chapter 3) and imaginary for absorptive media. Consider now an absorptive layer placed in a host medium, the coupling coefficient $m$ is related to the impedance mismatch between them. If the coupling coefficient from medium 1 to medium 2 is $r_{12}$ and $r_{21}=-r_{12}$, and the same acoustic 
thickness (or equivalently, the optical path, etc.) $d$ is considered for both materials, the coupling coefficient reads

$$
m=\frac{r_{12}-r_{21}}{d}=\frac{2 r_{12}}{d} .
$$

For the case of an acoustic wave:

$$
r_{12}=\frac{Z_{2}-Z_{1}}{Z_{2}+Z_{1}}
$$

where $Z_{i}$ stands for the acoustic impedance of the i-th medium.

Let consider now solutions of Eqs. 4.2 exponentially growing/decaying oscillating waves, $A(x), B(x)=e^{\lambda x}$, where $\lambda$ represent the complex eigenvalues of the matrix of the coefficients of Eqs. (4.2)

$$
\begin{gathered}
M=\left(\begin{array}{cc}
i \Delta k+\gamma & m \\
-m & -i \Delta k+\gamma
\end{array}\right), \\
\lambda_{ \pm}= \pm \sqrt{(\gamma+i \Delta k)^{2}+m^{2}} .
\end{gathered}
$$

Full solution for forward and backward propagating waves contain the integration constants $c_{1}, c_{2}$ :

$$
\begin{aligned}
& A(x)=c_{1} e^{\lambda x}+c_{2} e^{\lambda x} \\
& B(x)=c_{1} \frac{\lambda-(i \Delta k+\gamma)}{m} e^{\lambda x}+c_{2} \frac{-\lambda-(i \Delta k+\gamma)}{m} e^{\lambda x}
\end{aligned}
$$

For a finite system of length $L$, formed by $N$ layers, transmission and reflection coefficients can be obtained analytically by imposing boundary conditions at the entrance face $(x=0)$ for the forward field, $A(x=0)=1$, and at the rear face $(x=L)$ for the backward field $B(x=L)=0$. This leads to

$$
\begin{aligned}
T & =\frac{\lambda}{\lambda \cosh (\lambda L)-(\gamma+i \Delta k) \sinh (\lambda L)} \\
R & =\frac{m \sinh (\lambda L)}{\lambda \cosh (\lambda L)-(\gamma+i \Delta k) \sinh (\lambda L)}
\end{aligned}
$$

with $\lambda$ given by Eq. (4.6) with the negative sign (physical solutions of the problem). 


\subsubsection{Enhanced transmission in dissipative media}

The behavior of conservative periodic materials $(\gamma=0)$ at frequencies belonging to the band gap (around the Bragg frecuency $f_{b}=\pi / 2 a$ ) is well-known. The destructive interference occuring due to Bragg resonances at this frequencies cause incident waves to be efficiently back reflected. In terms of the transmission and reflection coefficients, shown in Eqs. (4.9), (4.10) by solving the CMT equations, this effect results in a decrease of the former and corresponding increase of the latter, as shown in Fig. 4.1(a).
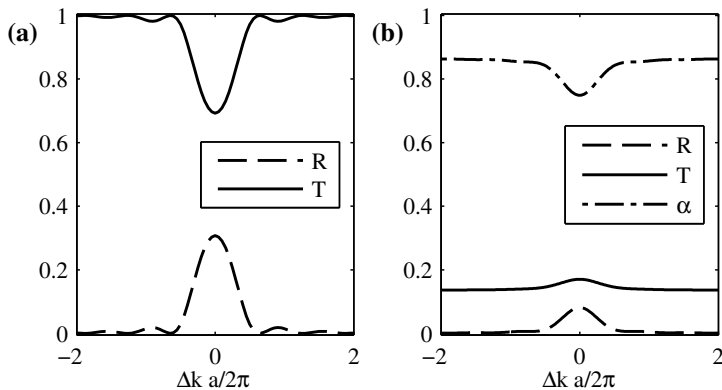

(c)

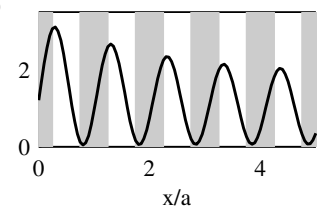

(d)

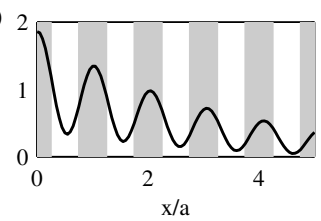

Figure 4.1: Transmission (solid line) reflection (dotted line) and absorption (dash-dotted line) spectra for waves in a periodic structure ( 5 periods, $L / a=5$ ) as calculated from Eqs. (4.9) and (4.10) for (a) conservative system (with coupling $m a=0.125$ and no losses $\gamma a=0$ ). (b) periodic system (with pure imaginary coupling valued $m a=i 0.125$ and losses $\gamma a=-0.2)$ predicting the anomalous transmission. (c) and (d) show the total intensity at the Bragg frequency, $\Delta k=0$, for the configurations shown in (a) and (b) respectively.

The situation is different when losses come into play. Consider an acoustic wave impinging a periodic lossy material formed by $N$ layers of purely absorptive material embedded in a host medium. Let the acoustic impedance of the absorber have the same real part as the host medium and a non-null imaginary part (i.e. pure imaginary $m$ and negative $\gamma$ ). Such a case is analogous to that considered for photonics in [Erhokhin et al., 2008] and [Kumar et al., 2012] and is depicted in Fig. 4.1(b), where the transmission (solid line), reflection (dashed line) and absorption (dash-dotted line) coefficients are represented. It is noted that, by energy balance, the absorption coefficient is calculated as 
$\alpha=1-\left|R^{2}\right|-\left|T^{2}\right|$. In this case, the reflection is increased at frequencies around the Bragg frequency, as expected. However, an anomalous transmission is observed at these range of frequencies, being maximum at the Bragg resonance $(\Delta k=0)$. The origin of such anomalous phenomenon is explained in Figs. 4.1(c) and 4.1(d), where the field distribution along the structure is shown for conservative and lossy cases at the Bragg frequency $(\Delta k=0)$, respectively. For a purely absorbent structured material, at these frequencies, the total field within the structure partially forms a standing wave, with the nodes of the particle velocity (maximum values of the acoustic field) located precisely inside the absorbing medium. As the nodes correspond to low particle velocity, there is few energy to be absorbed. As a consequence, such a configuration results in smaller absorption: both forward and backward waves are less absorbed, and the overall transmission is increased, as shown in Fig. $4.1(\mathrm{~b})$.

\subsubsection{Experimental study}

The predictions of anomalous transmission observed solving the dissipative coupled-mode equations are tested experimentally. It is worthy to note that the dissipative CMT used previously to illustrate this effect is independent of the particular type of wave. Hence, the coupling and gain (loss) coefficients are generic and do not represent the specifical physical parameters of a system in particular. Our interest here is the study of sound waves propagating through a finite system of periodically spaced porous layers embedded in a fluid medium (air). Thus, the experimental study has to be divided in two different stages: the first one involves the experimental evaluation of the reflection and absorption coefficients of the porous material. Once the material is properly characterized, the second step consist in the realization of an experiment designed to check the predictions of the anomalous transmission in a periodic structure formed by porous layers.

\section{Porous material characterization}

The porous material is characterized experimentally following the standard ISO-10534-2 (see Fig.4.2(a) for a schematic diagram), which specifies the methodology to determine the sound absorption coefficient in impedance tubes following the transfer-function method (TFM) for a single porous layer of a given thickness [Chung and Blaser, 1980a, Chung and Blaser, 1980b]. The 
physical parameters of the porous material are shown in Tab. 4.1. These parameters are used to evaluate the reflection and absorption coefficients of the porous layer using the analytical transfer-matrix method (TMM), described in detail in Ref. [Allard and Atalla, 2009]. These coefficients are illustrated in Fig. 4.2 for a single porous layer of thickness $D=8 \mathrm{~mm}$, showing a good agreement between analytical predictions (TMM), and experimental results using the standard ISO-10534-2. It is noted that the absorption of the porous material is quite low, thus the effective impedance of the porous layer is similar to that of the air. This constitutes a key factor with regard to a succesful realization of the experimental verification of the anomalous transmission effect in a periodic array of porous layers, as this material allows to measure the transmitted signals with an amplitude above the noise level, and at the same time posses enough losses to induce the anomalous properties expected in the layered media.

(a)
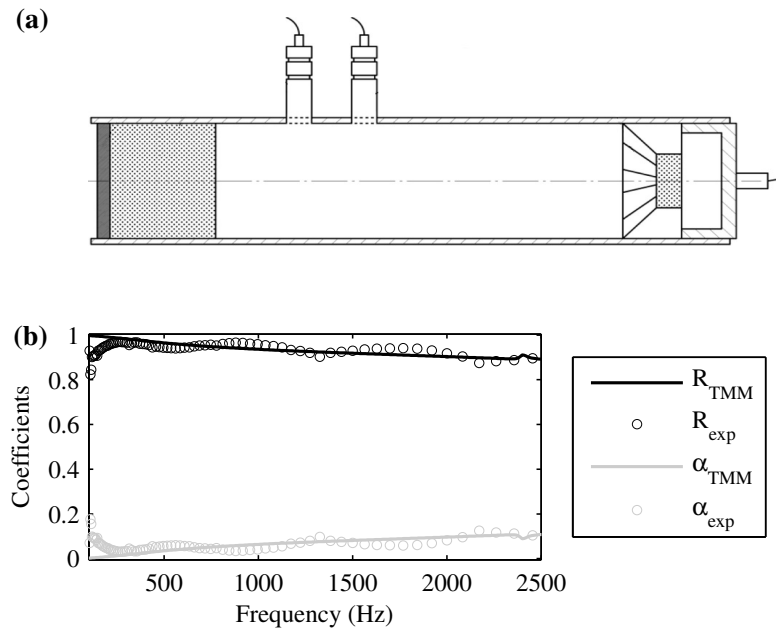

Figure 4.2: (a) Schematic diagram of the experimental setup for the characterization of the porous material using the standard ISO-10534-2. (b) Reflection, absorption coefficients of a single layer of porous material measured using the standard ISO-10534-2 (black, gray dots) calculated using TMM (black, gray solid lines).

\section{Periodic array of absorbing layers}

The layered material used in experiments is analytically characterized using the TMM. We consider here the most general case in which the frame of 


\begin{tabular}{cc}
\hline \hline Densitiy $\left(\mathrm{kg} / \mathrm{m}^{3}\right), \rho$ & 50 \\
\hline Porosity, $\phi$ & 0.97 \\
\hline Young's Modulus $(\mathrm{kPa}), E$ & 150 \\
\hline Poisson's coefficient & 0.35 \\
\hline Tortuosity, $\alpha_{\infty}$ & 1 \\
\hline Flow resistivity, $\sigma$ & 13000 \\
\hline Characterisitic length $(\mathrm{m}), \Lambda$ & $120 \times 10^{-6}$ \\
\hline \hline
\end{tabular}

Table 4.1: Physical parameters of the porous material used in the experiments and numerics.

(a)

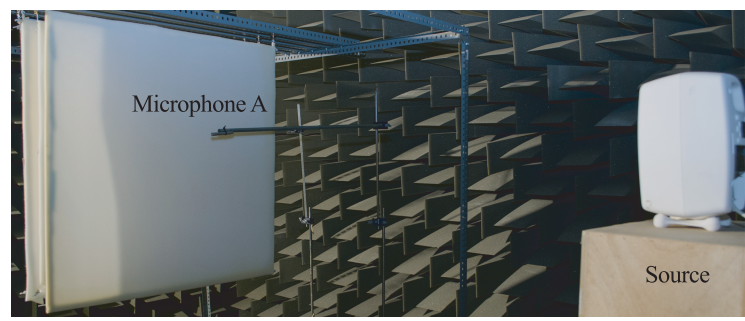

(b)

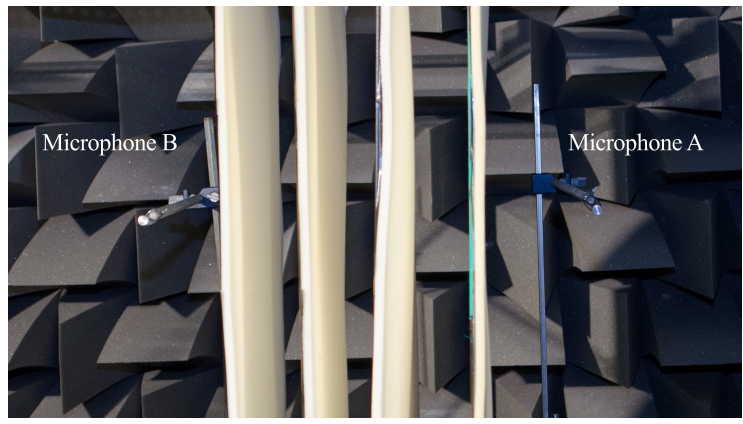

Figure 4.3: (a) Experimental set-up, consisting in an array of four plates of porous material; showing the source, a loudspeaker located in front of the structure, and the microphone to measure intensity at either side of the structure. (b) View of the system from a different angle. 
the porous material presents an elastic behaviour, so Biot's theory can be used to characterize the porous material. The layered structure is assumed to be laterally infinite (1D) and made of homogeneous and isotropic porous layers embedded in air. The transfer matrices are calculated assuming two compressional waves and one shear wave in the porous medium and one compressional wave in the fluid medium. All these waves are coupled by the boundary conditions and the result is a global transfer matrix which gives the propagation properties of the stratified media made of $N$ layers, and in particular its reflection and transmission coefficients.

The experimental set-up consists of a set of 4 parallel porous layers of $D=8 \mathrm{~mm}$ thickness embedded in air, as shown in Fig.4.3. The lattice constant is selected as $a=20 \mathrm{~cm}$. The acoustic source was placed in front of the first layer and two microphones A, B, were used to record the transmitted and reflected signals (placed in front and behind the first and last layer of the array, respectively). All the measurements were conducted in an anechoic chamber in order to avoid unwanted reflections. The range of frequencies of the measurements and the distance separating the source and the first layer have to be chosen with caution, as the system is modelled as one-dimensional, thus the wave field impinging the structure must be planar. With this regard, the acoustic source (Genelec 8030A commercial loudspeaker with a radius $r=6.35 \mathrm{~cm})$ can be roughly approximated as a circular baffled piston. The highest frequency considered is $f_{\max }=2500 \mathrm{~Hz}$ ensuring a nearly omnidirectional directivity pattern of the radiation, and the loudspeaker is placed at a sufficient distance (over $2 \mathrm{~m}$ ) in such a way that plane waves propagate through the periodic system.

Reflection, transmission and absorption coefficients are calculated from the acoustic pressure measurements registered by microphones $\mathrm{A}, \mathrm{B}$, in both sides of the periodic structure. The spectral characteristics were measured using the above described experimental scheme. The intensity coefficients were determined experimentally by measuring the sound field before (reflection $R$ ) and after (transmission $T$ ) the structure. It is noted that the incident wave was measured without the structure and then removed from the measured reflected field.

Figure 4.4 illustrates the comparison between the numerical predictions, obtained by applying the TMM and the experimental results, in good agreement. As predicted previously by the CMT, the usual increase of the reflection in the band-gap and the anomalous increase (decrease) of the transmission 
(absorption) at frequencies around the band gap $\left(f_{B}=850 \mathrm{~Hz}\left(f_{B}=1700 \mathrm{~Hz}\right)\right.$ is observed, for the first (second) band gap).

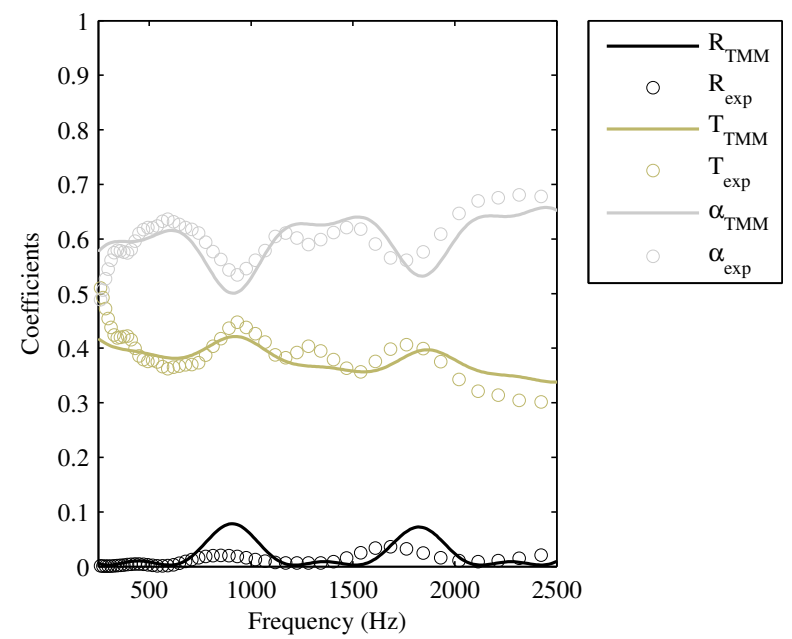

Figure 4.4: Reflection (black line/circles), transmission (gold line/circles) and Absorption (gray line/circles) of the periodic array of porous absorbers. Continuous lines represent theoretical predictions and circles represent experimental results.

The utilization of lossy materials in acoustics is usually aiming an opposite goal, which is the reduction of the wave energy, or specifically, reduction of noise. While a great variety of absorbing materials with different properties are already available, the design of acoustic insulators capable of absorbing sound waves in a broadband frequency range, for any direction of incidence and little material is still a great technological challenge. In the next section, a structured material fabricated out of porous lamellas, designed for that purpose, is presented.

\subsection{Extraordinary absorption of sound in porous lamella-crystals}

The concept of enhanced absorption in lossy lamella crystals is related to the time delay of sound waves propagating inside the system. For a bulk porous material $(p)$ of length $L$, backed by a perfect reflector, the acoustical path becomes $2 L$ and consequently the time delay is $\tau_{p}=2 L / c_{p}$ where $c_{p}$ is the sound speed in the porous material. In contrast, for a porous periodic crystal 
composed of the same base material (see Fig.4.5 for a schematic diagram), $c_{p}$ is replaced by the group velocity $v_{g}$ associated with the phononic crystal dispersion relation. The enhancement factor is defined following considerations developed in the context of absorption in chaotic cavities [Beenakker and Brouwer, 2001]

$$
\gamma=\frac{\Gamma \tau}{\Gamma_{p} \tau_{p}},
$$

where $\Gamma, \Gamma_{p}$ are the absorption rates for the bulk porous material and the porous periodic crystal (i.e., lamella crystal), respectively. The absorption rate in an asymmetric system (i.e., supported by a rigid backing) is related to the complex reflection coefficient

$$
|r(w)|^{2}=1-2 \Gamma \tau,
$$

The enhancement factor expresses the acoustic interaction strength of the crystal, that is to say, enhanced absorption is achieved for $\gamma>1$, meaning the dissipation inside the crystal exceeds the intrinsic material losses of the porous layer. In other words, for sufficiently long time delay, the reflectance can be minimal and the absorption extremely high, even for a modest absorption rate. Thus, from Eq. (4.12) it follows that reflectance (absorption) will decrease (increase) whenever wave slowing or increased dissipation is encountered. The reflection coefficient is calculated numerically simulating the complex wave interaction by coupling free-space sound radiation to Bloch-states inside the crystal, which is done using a plane wave expansion technique that comprises in-plane Bloch waves and out-of-plane harmonic waves.

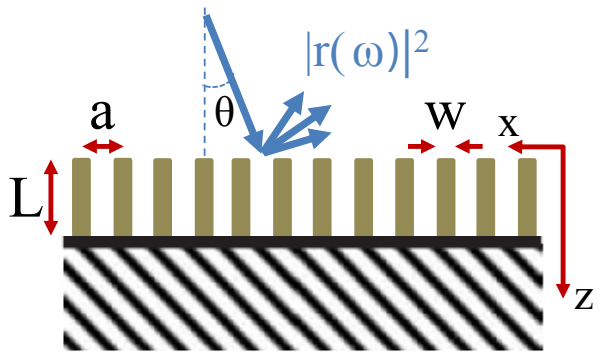

Figure 4.5: a Schematic of the crystal made of lamellas of width $W$, lattice constant $a$ and length $L$. The crystal is backed by a rigid support into which no sound waves penetrate.

Specific details on the theorical methods, as well as numerical simulations performed to analyze the behaviour of the structure, or the influence of the 
filling fraction and length of the porous layers in the absorption of the system, are beyond the scope of this section. Hence, the reader is referred to Appendix A.6 for a detailed description of the formulation. We will concentrate here in the description of experimental measurements and results.

\subsubsection{Experimental verifications}

\section{Experimental setup}

As mentioned in Section 4.1.3, the characterization of the reflection and absorption coefficients of a porous material is usually performed in the impedance tube with a rigid backing, following the TFM, described in the standard ISO10534-2. However, taking into account the size and configuration of the designed structure (see Fig.4.6), this methodology results rather impractical in this particular case. Instead, the characterization is made using a modified TFM applied in an anechoic chamber. Thus, the experiments account for a first stage where the reflectance and absorption coefficients for a porous material of a given thickness are characterized in an anechoic chamber for a normal incident acoustic wave, and compared to the same material measured in the impedance tube, with the aim of validating the experimental characterization in the anechoic chamber. Once the methodology is validated, the lamella crystal is characterized in the anechoic chamber following the modified TFM.

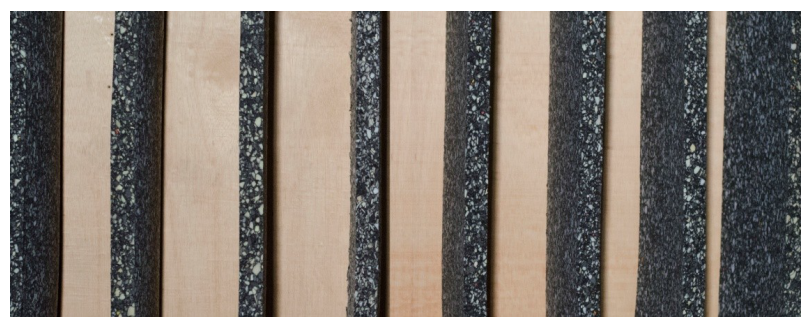

Figure 4.6: Bottom view on the suspended crystal made out of porous foam lamellas and supported by a rigid backing. The lamellas have the width $W=4 \mathrm{~cm}$ and length $L=0.5 \mathrm{~m}$.

The lamella crystal used in the experiments is backed by a rigid wood panel, containing 9 unit cells of lamellas of width $W=4 \mathrm{~cm}$, length $L=50$ $\mathrm{cm}$ and depth $D=100 \mathrm{~cm}$ that are mounted upside down without weight suspensions, as depicted in Fig.4.6. The filling fraction of the lamella crystal is $f f=0.36$. The performance of the lamella crystal for different incident angles of the acoustic wave is also studied. For this purpose, the crystal was 
mounted on a rotatable frame with a vertical axis. For the sound excitation we use a Genelec $8130 \mathrm{~A}$ loudspeaker placed at a distance of $d=2 \mathrm{~m}$ to the first microphone to ensure propagation of plane waves for the entire range of frequencies analysed. Two B\&K 4189 microphones of 1/2 inch are placed at the equatorial plane of the crystal and aligned with the central unit cell. Data from the two microphones are acquired with a CLIO $10 \mathrm{FW}$ (Audiomatica) and then processed in a computer.

\section{TFM applied in anechoic chamber}

We verify the use of the modified TFM in the anechoic chamber and select a specific range of frequencies to be compared to measurements in the impedance tube. The TFM used for measuring in-duct acoustic properties of materials is a well-known method for the determination of the absorption and acoustic impedances [Chung and Blaser, 1980a, Chung and Blaser, 1980b]. Using this method, the complex reflection coefficient reads as follows

$$
r(\omega)=\frac{H_{12}-H_{1}}{H_{2}-H_{12}} e^{i 2 k_{0} l},
$$

where $k_{0}$ is the wave number in air and $l$ is the distance from the last microphone position to the sample as shown in Fig.4.7(a). The acoustic transfer functions expressed in Eq.(4.13) are written as

$$
\begin{aligned}
H_{1} & =e^{-i k_{0} s}, \\
H_{2} & =e^{i k_{0} s}, \\
H_{12} & =\frac{p_{1} e^{i \theta_{1}}}{p_{2} e^{i \theta_{2}}},
\end{aligned}
$$

where $p_{i}$ and $\theta_{i}$ are the amplitude and the phase, respectively, measured at the position $i$, following the scheme shown in Fig.4.7(a). The absorbing material is placed at the end of the tube and supported by a rigid backing which leads to the simple expression for the absorption $A=1-|r(w)|^{2}$. This method is widely used in acoustics to characterize bulk absorbing materials.

The experimental set-up for the adapted method is illustrated in Fig.4.7(b). Notice that the distribution of the elements is the same as in the case of the impedance tube (Fig.4.7(a)). To ensure plane wave generation, the distance between the source and the first microphone should be long enough for the range of frequencies analysed. Moreover, to avoid the finite size effects of the 
(a)

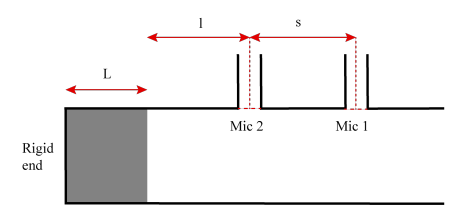

(b)

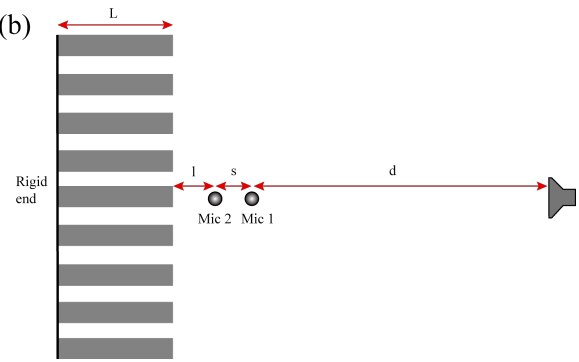

Figure 4.7: Schematic diagrams of the experimental set-up for the experimental characterization of reflection and absorption coefficients in (a) the impedance tube and (b) the anechoic chamber.

sample, the distance $l$ between the sample and the second microphone should be sufficiently small. Notice also that the periodic crystal is mounted onto a rigid end. This rigid end is a wood panel, which is characterized later in this section.

The porous lamella crystal studied in this work is made out of sheets of a homogeneous foam, consisting of a mixture of polyurethane, polyester and polyether, compacted and compressed. The exact prediction of the effective density and dynamic modulus of the saturating fluids in real porous materials is generally rather difficult because of the very complicated pore geometries. However, in the case where the foam is considered as a porous material saturated with a Newtonian fluid that is incompressible on the scale of the pore size, several models are readily available to determine effective acoustic parameters used for the complex scattering coefficients [Lafarge et al., 1997].

Fig.4.8(a) illustrates the reflectance $|r(w)|^{2}$ and the absorption $A$ of a sample of foam of length $L=4 \mathrm{~cm}$ measured in the impedance tube using the TFM and predicted theoretically. The discrepancies at high frequency appear when approaching the cut-off of the tube. Based on the same technique, we also conducted measurements in the anechoic chamber of a foam sheet of 1 $\mathrm{m}^{2}$ and with a length $L=4 \mathrm{~cm}$. This sheet is placed over the rigid end following the scheme of Fig.4.7(b). In Fig.4.8(b) we plot the reflectance and absorption measured in the anechoic chamber, which are comparable with the corresponding results in the impedance tube. With this comparison we have validated the feasibility of the TFM in the anechoic chamber over a selected range of frequencies.

We also characterize the wood panel used as a rigid support in the experi- 

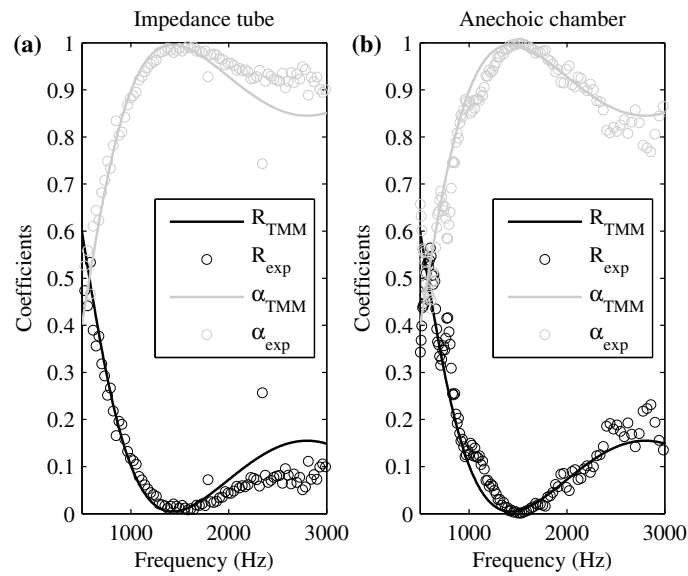

Figure 4.8: Theoretical and experimental characterization of a sample of porous mateiral with length $L=4 \mathrm{~cm}$ obtained by the TFM in (a) the impedance tube and (b) the anechoic chamber.

mental set-ups. Frequency independent full reflectance and zero absorption is measured for sound irradiating the panel, validating the assumption of a rigid backing, as observed in Fig.4.9(a). Additionally, no spectral phase contributions (evaluated from the complex reflection coefficient) are observed when waves are fully reflected at the panel, as shown in Fig.4.9(b).
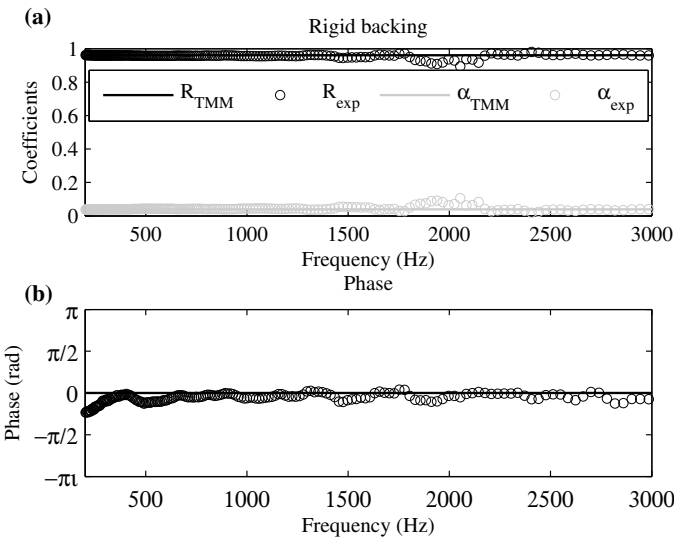

Figure 4.9: Reference measurements of the rigid end. (a) Theoretical (solid lines) and experimental (dots) reflectance $|r(w)|^{2}$ (gray) and absorption $A$ (black). (b) Theoretical (solid line) and experimental (dots) evaluation of the phase of the reflection coefficient. 


\section{Experimental results}

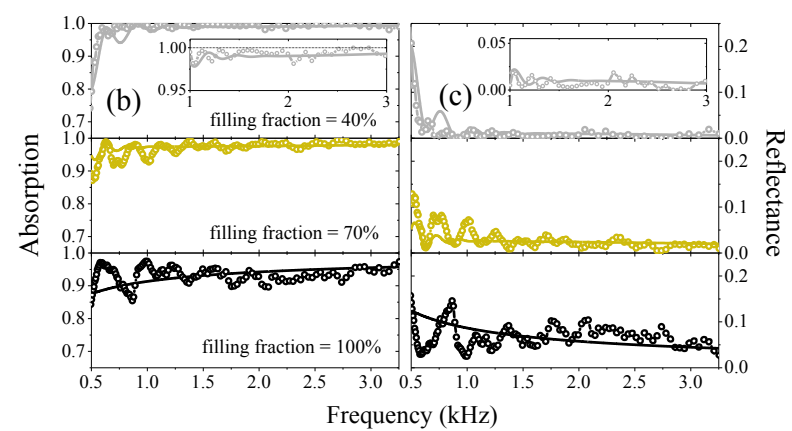

Figure 4.10: (b,c) Measured (open circles) and simulated (full lines) absorption and reflectance versus frequency for three different filling fractions, $f=40 \%, 70 \%$ and $100 \%$. All data are obtained for a normal incident acoustic plane wave.

From the numerical study (see Appendix A.6), it is predicted that lowering the crystal filling fraction will improve the absorption of sound due to the enhancement of the interaction strength. To validate this experimentally we have constructed various samples made out of the same lamellas with three different filling fractions, varying the size of the unit cell. Results are depicted in Fig.4.10. Bulk material properties are obtained measuring the response of the structure with a filling fraction of $100 \%$ from which relatively strong absorption stems from intrinsic material losses and the slab length $L$. When lowering the filling fraction down to $70 \%$ and $40 \%$, we observe increased performances with mean absorption of 0.97 and 0.99 , respectively, over an extended spectral range spanning from 0.7 to $3 \mathrm{kHz}$. Due to a finite number of unit cells we detect oscillations in the spectrum, overall however, the theory agrees very well with the average absorption evaluated from experiments.

Due to the interaction strength exceeding unity, complete sound absorption extends much further away from normal incidence for various directions. We conducted angular-resolved absorption measurements where we vary the angle $\theta$ from the normal to the crystal surface. Caused by diffraction associated by momentum transfer to the lattice (dash-dotted lines), regions of higher reflections are predicted, as seen in Fig.4.11 (see Appendix A.6 for a detailed explanation on the origin of these reflections). This narrow region is however being surpassed by a spectrally broad region of strong absorption spanning from $\theta=0^{\circ}-40^{\circ}$. Both, the finite number of unit cells and the rigid rotatable frame used for the angular measurements are causing additional un- 


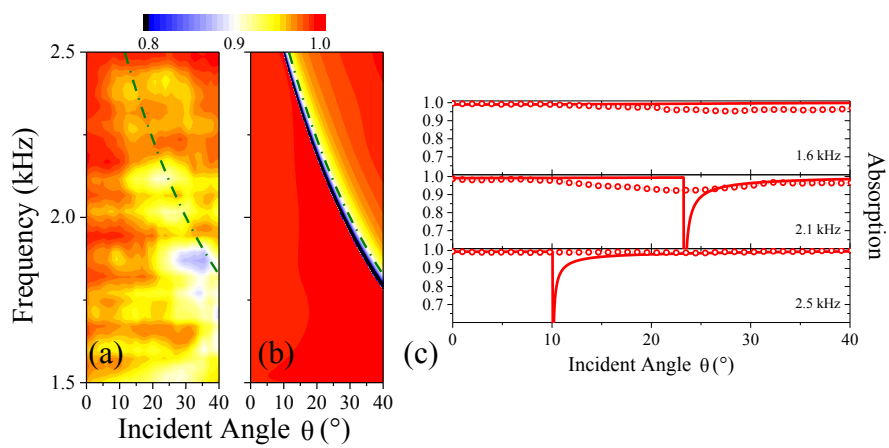

Figure 4.11: Measured and simulated angle-resolved absorption spectrum. (a) Measured and (b) simulated absorption as a function of frequency and angle of incidence for the same structure as in the previous measurements, now just with $f=36 \%$. The dash-dotted line on both contour-maps indicate the condition where $\left|k_{x}+G_{x}\right|=\omega / c_{0}$. (c), Angular response of the absorption, representing three measured (open circles) and simulated (full lines) frequency cuts from the contours in (a) and (b).

wanted reflections in the measurements, as observed in Fig.4.11(a). However, upon inspecting both experimentally and numerically the absorption for three different frequencies within the entire resolved spectra, we find overall good agreement validating broadband absorption for almost any direction as seen in Fig.4.11(c).

In section 4.1 an anomalous phenomena related to periodic structures formed by absorptive materials is described and measured experimentally for the case of sound waves. The study indicates an enhancement and reduction of transmission and absorption at frequencies around the band gaps, which is described by the dissipative CMT equations showing the differences encountered when comparing to the well known reflection and transmission from conservative periodic structures. On the other hand, experimental results are in good agreement with TMM analytical predictions.

The concept of enhanced absorption in porous lamella crystals is described in section A.6. A modified TFM is firstly verified by comparison to the standard TFM in the impedance tube, and then applied to measure a system made out of lossy lamellas mounted onto a rigid backing in the anechoic chamber, demonstrating the ability to increase absorption by lowering the effective amount of material, not only for normal incident radiation but for all-angle incidence, and for a broadband frequency range. Conclusions will be presented in Chapter 6 


\section{References}

Allard, J. and Atalla, N. (2009). Propagation of sound in porous media: modelling sound absorbing materials 2e. John Wiley \& Sons.

Andreassen, E. and Jensen, J. S. (2013). Analysis of phononic bandgap structures with dissipation. Journal of Vibration and Acoustics, 135(4):041015.

Beenakker, C. and Brouwer, P. (2001). Distribution of the reflection eigenvalues of a weakly absorbing chaotic cavity. Physica E: Low-dimensional Systems and Nanostructures, 9(3):463-466.

Botten, L., Craig, M., McPhedran, R., Adams, J., and Andrewartha, J. (1981). The finitely conducting lamellar diffraction grating. Journal of Modern Optics, 28(8):1087-1102.

Chung, J. and Blaser, D. (1980a). Transfer function method of measuring in-duct acoustic properties. i. theory. The Journal of the Acoustical Society of America, 68(3):907-913.

Chung, J. and Blaser, D. (1980b). Transfer function method of measuring in-duct acoustic properties. ii. experiment. The Journal of the Acoustical Society of America, 68(3):914-921.

Clapham, P. and Hutley, M. (1973). Reduction of lens reflexion by the moth eye principle.

Erhokhin, S. G., Lisyansky, A. A., Merzlikin, A. M., Vinogradov, A. P., and Granovsky, A. B. (2008). Photonic crystals built on contrast in attenuation. Phys. Rev. B, 77:233102.

Figotin, A. and Vitebskiy, I. (2008). Absorption suppression in photonic crystals. Phys. Rev. B, 77:104421.

Figotin, A. and Vitebskiy, I. (2010). Magnetic faraday rotation in lossy photonic structures. Waves in Random and Complex Media, 20(2):298-318.

García-Chocano, V. M., Cabrera, S., and Sánchez-Dehesa, J. (2012). Broadband sound absorption by lattices of microperforated cylindrical shells. Applied Physics Letters, 101(18):184101.

Garcia-Vidal, F., Pitarke, J., and Pendry, J. (1997). Effective medium theory of the optical properties of aligned carbon nanotubes. Physical review letters, 78(22):4289.

Garcia-Vidal, F. J. (2008). Metamaterials: Towards the dark side. Nature Photonics, $2(4): 215-216$.

Hussein, M. I. (2009). Theory of damped bloch waves in elastic media. Phys. Rev. B, $80: 212301$.

Hwan Oh, J., Jae Kim, Y., and Young Kim, Y. (2013). Wave attenuation and dissipation mechanisms in viscoelastic phononic crystals. Journal of Applied Physics, 113(10).

Kravets, V. G., Schedin, F., and Grigorenko, A. (2009). Almost complete absorption of light in nanostructured metallic coatings: Blackbody behavior. Proc. PIERS, Moscow, Russia, 79.

Kumar, N., Botey, M., Herrero, R., Loiko, Y., and Staliunas, K. (2012). High-directional wave propagation in periodic loss modulated materials. Photonics and Nanostructures Fundamentals and Applications, 10(4):644 - 650. TaCoNa-Photonics 2011.

Kumar, N., Herrero, R., Botey, M., and Staliunas, K. (2013). Flat lensing by periodic loss-modulated materials. J. Opt. Soc. Am. B, 30(10):2684-2688. 
Lafarge, D., Lemarinier, P., Allard, J. F., and Tarnow, V. (1997). Dynamic compressibility of air in porous structures at audible frequencies. The Journal of the Acoustical Society of America, 102(4):1995-2006.

Laude, V., Escalante, J. M., and Martínez, A. (2013). Effect of loss on the dispersion relation of photonic and phononic crystals. Phys. Rev. B, 88:224302.

Lee, C.-Y., Leamy, M. J., and Nadler, J. H. (2010). Frequency band structure and absorption predictions for multi-periodic acoustic composites. Journal of Sound and Vibration, 329(10):1809 - 1822 .

Leroy, V., Bretagne, A., Fink, M., Willaime, H., Tabeling, P., and Tourin, A. (2009a). Design and characterization of bubble phononic crystals. Applied Physics Letters, 95(17):171904.

Leroy, V., Strybulevych, A., Scanlon, M., and Page, J. (2009b). Transmission of ultrasound through a single layer of bubbles. The European Physical Journal E, 29(1):123-130.

Lidorikis, E. and Ferrari, A. C. (2009). Photonics with multiwall carbon nanotube arrays. ACS nano, 3(5):1238-1248.

McPhedran, R., Botten, L., Craig, M., Neviere, M., and Maystre, D. (1982). Lossy lamellar gratings in the quasistatic limit. Journal of Modern Optics, 29(3):289-312.

Mei, J., Ma, G., Yang, M., Yang, Z., Wen, W., and Sheng, P. (2012). Dark acoustic metamaterials as super absorbers for low-frequency sound. Nature Communications, 3:756.

Mizuno, K., Ishii, J., Kishida, H., Hayamizu, Y., Yasuda, S., Futaba, D. N., Yumura, M., and Hata, K. (2009). A black body absorber from vertically aligned single-walled carbon nanotubes. Proceedings of the National Academy of Sciences, 106(15):6044-6047.

Psarobas, I. E. (2001). Viscoelastic response of sonic band-gap materials. Phys. Rev. B, 64:012303.

Romero-García, V., Sánchez-Pérez, J., and Garcia-Raffi, L. (2011). Tunable wideband bandstop acoustic filter based on two-dimensional multiphysical phenomena periodic systems. Journal of applied physics, 110(1):014904.

Søndergaard, T., Novikov, S. M., Holmgaard, T., Eriksen, R. L., Beermann, J., Han, Z., Pedersen, K., and Bozhevolnyi, S. I. (2012). Plasmonic black gold by adiabatic nanofocusing and absorption of light in ultra-sharp convex grooves. Nature communications, $3: 969$.

Staliunas, K., Herrero, R., and Vilaseca, R. (2009). Subdiffraction and spatial filtering due to periodic spatial modulation of the gain-loss profile. Phys. Rev. A, 80:013821.

Thomas, E. L. (2009). Applied physics: Bubbly but quiet. Nature, 462(7276):990-991.

Tournat, V., Pagneux, V., Lafarge, D., and Jaouen, L. (2004). Multiple scattering of acoustic waves and porous absorbing media. Phys. Rev. E, 70:026609.

Yang, Z.-P., Ci, L., Bur, J. A., Lin, S.-Y., and Ajayan, P. M. (2008). Experimental observation of an extremely dark material made by a low-density nanotube array. Nano letters, 8(2):446-451. 


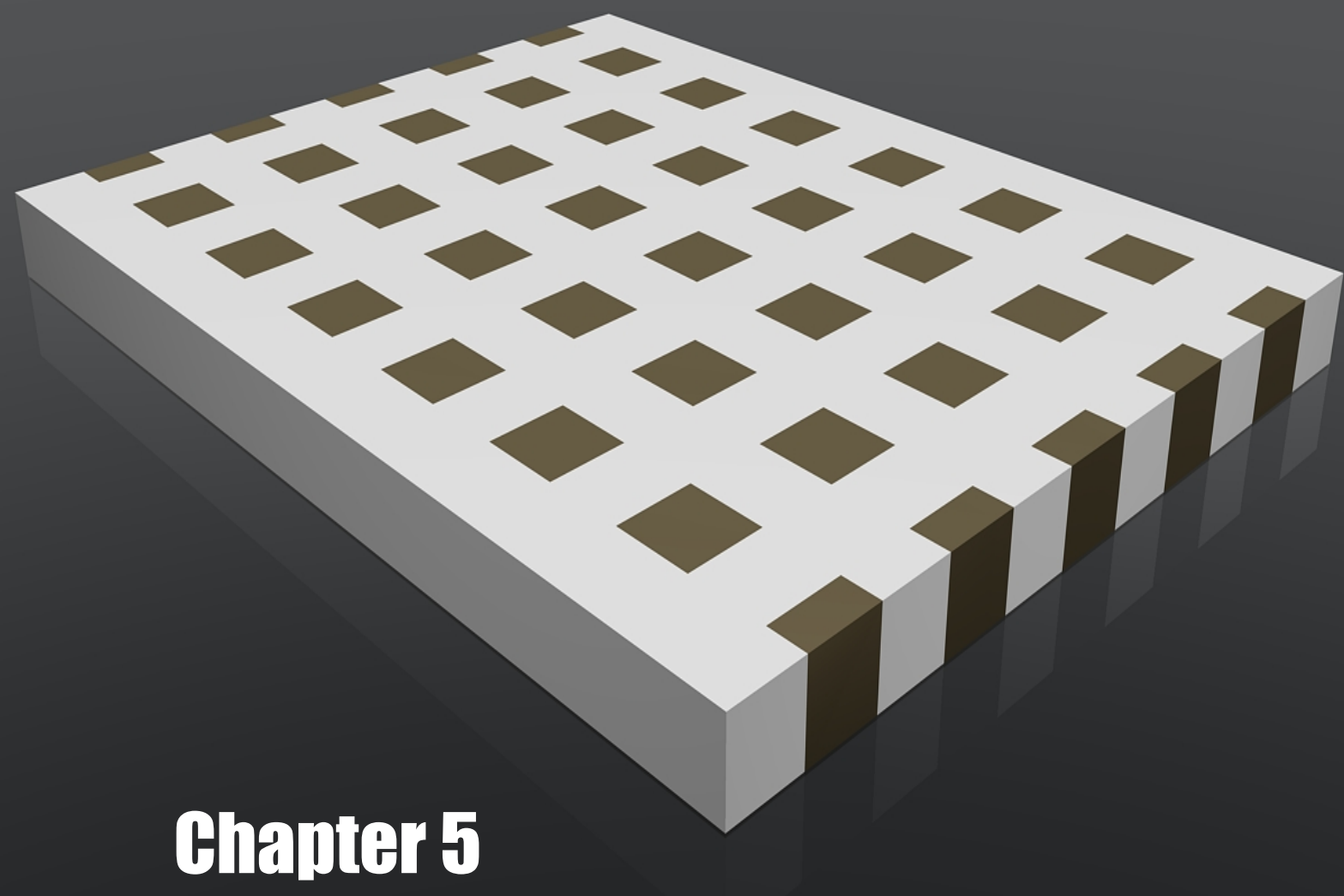

\section{Band structure calculations in time-domain}

In this chapter we present a methodology for elastic band structure and Bloch mode shape calculations in time-domain. We describe the discretization of the equations of motion in space, using the finite element method, and time, using an explicit Newmark method. The model accounts for a single unit cell by implementing Bloch boundary conditions. By applying a wideband excitation signal the resonant modes in the unit cell are excited and the conditions set for the analysis of the time-history data, leading to the computation of the band structures and the Bloch mode shapes. The performance of the method is analyzed in terms of accuracy, convergence and computation time and compared to the results obtained by solving an eigenvalue problem using the finite element method. Finally, the usefulness of the method for a particular application is briefly discussed. 
The band structure is the main characterization tool to understand wave propagation characteristics in periodic materials as it represents the dispersion properties of waves in periodic media. A great effort has been made over the last few decades on the development of techniques for band structure calculations. These include plane wave expansion (PWE) [Ho et al., 1990, Meade et al., 1993, Johnson and Joannopoulos, 2001, Kushwaha et al., 1993, Sigalas and Economou, 1993], the transfer matrix method (TMM) [Pendry and MacKinnon, 1992], multiple scattering theory (MST) [Leung and Qiu, 1993, Wang et al., 1993, Kafesaki and Economou, 1999, Kafesaki et al., 2000, Liu et al., 2000], the finite-element method (FEM) [Axmann and Kuchment, 1999,Dobson, 1999,Pask et al., 2001], the finite-difference (FD) method [Yang, 1996], and the finite-difference time-domain method (FDTD) [Ward and Pendry, 1998, Qiu and He, 2000, Tanaka et al., 2000, Cao et al., 2004,Hsieh et al., 2006], among others [Jun et al., 2003, Moreno et al., 2002, Checoury and Lourtioz, 2006, Yan and Wang, 2006, Chiang et al., 2007]. Reviews of periodic materials and band structure calculations methods can be found in [Busch et al., 2007, Hussein et al., 2014] for photonic and phononic crystals, respectively.

Most of these methods assume steady-state solutions (i.e., harmonic time dependence) and account for Bloch theory [Kittel and Holcomb, 1967], allowing the calculation of the band structure to be performed over a single unit cell. The dispersion relation of the periodic system is obtained after solving an eigenvalue problem, which is expressed in terms of a frequency versus wave vector diagram spanning the latter along the Brillouin zone (BZ). Among these methods, PWE has been extensively used for acoustic and elastic composites [Kushwaha et al., 1993, Sigalas and Economou, 1993] and it is appropriate for solid-solid and fluid-fluid composites, while FD methods are more suited for phononic systems composed of multiple states (e.g. solidfluid) [Garcia-Pablos et al., 2000]. However, both methods are not suitable when dealing with irregularly shaped scatterers. Other approaches, such as MST, first reported in [Kafesaki and Economou, 1999,Kafesaki et al., 2000,Liu et al., 2000] for phononic systems, are applicable to media composed of cylindrical/spherical scatterers in a host medium (in contrast to square inclusions in a host medium or layered phononic systems). This method can handle multiple media states, such as elastic scatterers in fluids or air holes in an elastic solid medium, and accurately analyze high-contrast problems, where other methods (PWE, FD, FE) may exhibit slow convergence. Nevertheless, the geometry of the unit cell is highly restricted. Ultimately, FE has been 
employed as an efficient method for band structure calculation in phononic systems by formulating an eigenvalue problem where Bloch boundary conditions are also enforced, thus considering a single unit cell. In the context of FE methods, an alternative approach to the usual implementation of Bloch boundary conditions [Krattiger and Hussein, 2014] is based on considering a Bloch operator transformation of the governing differential equations to obtain the strong form of the Bloch eigenvalue problem [Hussein, 2009]. A great advantage of $\mathrm{FE}$ over the previous methods is the ease to deal with complex geometries.

While the aforementioned methods assume harmonic time dependence to solve the governing elastic wave equation, some authors have reported on band structure calculation by computing the time-domain response. Specifically, FDTD method, where the governing equation is discretized in both space in and time, has been used for that purpose. The time-history data resulting from the simulation is Fourier-transformed into the frequency domain to get the dispersive characteristics of the medium. However, if Bloch boundary conditions are not imposed, the computational domain has to include many unit cells and a proper choice of boundary conditions must be realized [Garcia-Pablos et al., 2000]. By varying the source's excitation frequency, the dependence of attenuation (due to evanescence) with the frequency can be obtained and used to identify the bandgaps. Following this approach (i.e., using several unit cells) the band structure can be computed by analyzing phase differences at the input and the output of a finite-size structure [Robertson and Rudy III, 1998, Rubio et al., 1999]. In contrast, Tanaka et al. [Tanaka et al., 2000] presented an FDTD approach for phononic systems in which Bloch boundary conditions were imposed, thus reducing the simulation over a single unit cell, and the band structure was computed by varying the wave vector through the BZ, using the Fourier transforms of the time-history data.

The computational effort associated with band structure calculations is usually high because it involves solving a complex eigenvalue problem, in the case of harmonic time dependence, or a large number of time iterations, in case of time-domain methods. Moreover, this process is done numerous times as the value of the wave vector, $\mathbf{k}$, is varied along the BZ (or the Irreducible BZ (IBZ)). The size of the problem, and hence the computational load, is particularly high when the unit cell configuration requires a large number of degrees of freedom (DOF) to be properly described. This could be due to a complex unit cell material phase topology, requiring a finely resolved description. Another case is when the presence of defects is incorporated in the 
calculations. Defects are known to have a physical influence extending over very long ranges in space. This, in turn, requires choosing correspondingly large unit cells, known as supercells, for the band structure calculations. Consequently, large cells imply large numbers of DOF. The computational complexity of eigenvalue problems is usually higher than matrix inversion, which is a bottle-neck in terms of computational effort in time-domain algorithms. For a small to moderate number of DOF the former is far more efficient but, as it will be demonstrated here, for a certain number of DOF the latter will become more efficient.

In this chapter, a new methodology based on the finite-element in timedomain method (FETD) for elastic band structure calculation of 1D and $2 \mathrm{D}$ phononic crystals is presented. The proposed method implements a onedimensional model for longitudinal wave propagation and a two-dimensional plane-strain model of a phononic crystal that accounts for coupled in-plane longitudinal and shear vertical wave propagation modes. Band structure calculations are performed incorporating Bloch boundary conditions so that the space discretization of the domain is reduced to a single unit cell. A slight but highly efficient modification of the 2D method incorporated and analyzed in this work consists in the diagonalization of the mass matrix (i.e., lumped mass), as the computational efficiency of time domain integration methods is greatly improved if the process of matrix inversion is done using sparse matrices. The performance of the method is analyzed by computing the elastic band structures (1D, 2D) and Bloch mode shapes (2D) of a phononic crystal consisting of a beam lattice of aluminum and an ABS thermoplastic polymer in 1D, and square inclusions of aluminum embedded in a ABS matrix in 2D. Results are compared to the standard eigenvalue problem in FE, which is chosen as the reference method to evaluate the accuracy, convergence and computational efficiency of the proposed method.

\section{$5.1 \quad$ Unit-cell finite-element model}

The continuum equation of motion for a heterogeneous medium is

$$
\nabla \cdot \sigma=\rho \mathbf{u},
$$

where $\sigma$ is the stress tensor, $\mathbf{u}$ is the displacement field, $\rho$ is the density and dots indicate differentation with respect to time. For an elastic medium,

$$
\sigma=\mathbf{C}: \nabla^{S} \mathbf{u}
$$


where $\mathbf{C}$ is the elasticity tensor and $\nabla^{S}$ denotes the symmetric gradient operator,

$$
\nabla^{S} \mathbf{u}=\frac{1}{2}\left(\nabla \mathbf{u}+(\nabla \mathbf{u})^{T}\right) .
$$

Substituting Eq. (5.2) into Eq. (5.1) the strong form of the general elastodynamic problem is obtained,

$$
\nabla \cdot \mathbf{C}: \nabla^{S} \mathbf{u}=\rho \ddot{\mathbf{u}}
$$

\section{Finite-element discretization}

The FE method is used for the spatial discretization of the unit cell. The solution domain $\Omega$ is defined to span the range $0 \leq(x, y) \leq a$, where $a$ is the lattice constant. The strong form of the general elastodynamic problem in Eq. (5.4) is converted into the weak form introducing a weighting function w and integrating over the solution domain

$$
-\int_{\Omega}\left(\nabla^{S} \mathbf{w}: \mathbf{C}: \nabla^{S} \mathbf{u}\right) d \Omega=\int_{\Omega}(\rho \mathbf{w} \cdot \mathbf{u}) d \Omega .
$$

Note that a force term is not included as the loading is made in terms of prescribed displacement. The solution domain is discretized into $n_{e l}$ element domains $\Omega^{e}$,

$$
\Omega=\bigcup_{e=1}^{n_{l}} \Omega^{e} .
$$

A typical weighting function for an element in the domain has the form,

$$
\mathbf{w}_{1}=N_{A} \mathbf{w}_{1 A}, \quad A=1, n_{e n},
$$

where $N_{A}$ is a shape function associated with the node $A, w_{1 A}$ is the $A^{\text {th }}$ component of the approximate weighting function, and $n_{e n}$ is the number of element nodes. The time dependent displacement field is discretized in a similar way,

$$
\mathbf{u}=N_{B} \mathbf{d}_{1 B}, \quad B=1, n_{e n},
$$

where $\mathbf{d}_{1 B}$ is time dependent and express the nodal displacement. Substituting Eq. (5.7) and Eq. (5.8) into Eq. (5.5) yields

$$
-\int_{\Omega}\left(\nabla^{S} N_{A} \mathbf{w}_{1 A}: C: \nabla^{S} N_{B} \mathbf{d}_{1 B}\right) d \Omega=\int_{\Omega}\left(\rho N_{A} \mathbf{w}_{1 A} \cdot N_{B} \mathbf{d}_{1 B}\right) d \Omega .
$$


Equation (5.9) can be expressed as a system of algebraic equations in the following matrix form,

$$
-\mathbf{M U}+\mathbf{K U}=0,
$$

where $\mathbf{U}$ and $\mathbf{U}$ are the nodal displacement and acceleration, respectively. $\mathbf{M}$ and $\mathbf{K}$ are the mass and stiffness matrices, respectively, and are assembled from the element mass and stiffness contributions following the direct stiffness method,

$$
\begin{aligned}
\mathbf{M} & =\sum_{e=1}^{n_{e l}} \mathbf{M}^{e}, \\
\mathbf{K} & =\sum_{e=1}^{n_{e l}} \mathbf{K}^{e} .
\end{aligned}
$$

The time-evolving elastodynamic wave propagation can be analyzed by the time integration of Eq. (5.9). Together with the application of periodic Bloch boundary conditions and a proper transient excitation, this equation can be solved for different values of the Bloch vector to obtain the band structure of a periodic system.

\section{Bloch boundary conditions}

Bloch theory describes the behavior of a particle wavefunction in an infinite periodic medium in terms of wave functions at the reciprocal space [Kittel and Holcomb, 1967]. A function accomplishing this feature may be written as the product of the unit-cell Bloch function, which is periodic over the unit cell, and a periodic function having the same periodicity

$$
\mathbf{u}(\mathbf{x}, \mathbf{k} ; t)=\mathbf{u}(\mathbf{x}, \mathbf{k}) e^{i\left(\mathbf{k}^{T} \mathbf{x}-w t\right)},
$$

in a two-dimensional geometry, $\mathbf{x}=(x, y)$ is the position, $\mathbf{k}=\left(k_{x}, k_{y}\right)$ is the Bloch wave vector and $\mathbf{u}$ is the Bloch function. Consider now the unit cell represented in Fig. 5.1(a). The set of edge nodes belonging to the top and right edges (dashed lines in Fig.5.1(a)) constitute redundant DOF as they actually belong to neighbouring unit cells. Hence, these sets of DOF are removed by linking them to those DOF belonging to bottom and left edges (continuous lines in Fig. 5.1(a)). The equations representing boundary conditions are collected in matrix form and inserted into the mass and stifness matrices through a transformation matrix. Specific details on how these sets of DOF 
are defined, linked and inserted into the Bloch transformation matrix can be found in [Krattiger and Hussein, 2014].

\subsection{Unit-cell time-domain simulation}

\section{Time integration method}

Equation (5.9) is discretized in time using one of the several forms of Newmark's time-integration methods [Newmark, 1959], yielding in the computation of displacements, velocities and accelerations for increasing values of time, $t$. The subsequent system of equations obtained from Newmark's family of methods are

$$
\begin{gathered}
\mathbf{D}_{n+1}=\mathbf{D}_{i}+\Delta t \mathbf{V}_{i}+(\Delta t)^{2}\left[\left(\frac{1}{2}-\beta\right) \mathbf{A}_{i}+\beta \mathbf{A}_{i+1}\right] \\
\mathbf{V}_{i+1}=\mathbf{V}_{i}+\Delta t\left[(1-\gamma) \mathbf{A}_{i}+\gamma \mathbf{A}_{i+1}\right] \\
\mathbf{M A}_{i+1}+\mathbf{K D}_{i+1}=\mathbf{F}_{i+1} .
\end{gathered}
$$

In these equations, $\mathbf{D}, \mathbf{V}$ and $\mathbf{A}$ denote the vector displacement, velocity and acceleration, respectively. The time interval is denoted $\Delta t$ and $i$ indicates the time step. $\mathbf{M}$ and $\mathbf{K}$ are the mass and stiffness matrices, respectively. $\beta$ and $\gamma$ are the Newmark parameters determining the specific type of Newmark scheme, affecting stability and accuracy of the numerical simulation. For this work these parameters are chosen to be $\beta=0, \gamma=1 / 2$, providing an explicit center difference Newmark scheme, which is computationally efficient and less storage is required than for implicit methods. However, an explicit Newmark scheme is conditionally stable and thus, time interval is defined such that the Courant-Friedricks-Levy (CFL) lies at the stability limit

$$
\Delta t=\frac{\Delta h^{e}}{c_{\max }},
$$

where $\Delta t$ is the time interval, $\Delta h^{e}$ is the element size and $c_{\max }$ is the highest phase velocity of any of the media in the domain. For time intervals below this criterium some accuracy is compromised but stability is still guaranted.

The procedure for solving elastodynamic wave propagation using Newmark scheme is done as follows: given $\mathbf{K}, \mathbf{M}, \mathbf{F}$ and proper initial conditions 
for prescribed displacement, the following steps are needed to compute displacement, velocity and acceleration:

1. First step consist of obtaining acceleration for $t=0$ applying Eq. (5.16).

2. Time iteration starts to evolve and acceleration, displacement and velocity for future times are calculated. Eq. (5.16) is used to evaluate $\mathbf{A}_{i+1}$.

3. Then, future displacement $\mathbf{D}_{i+1}$ is obtained using Eq. (5.14).

4. Finally, Eq. (5.15) allows to determine $\mathbf{V}_{i+1}$.

\section{Transient excitation}
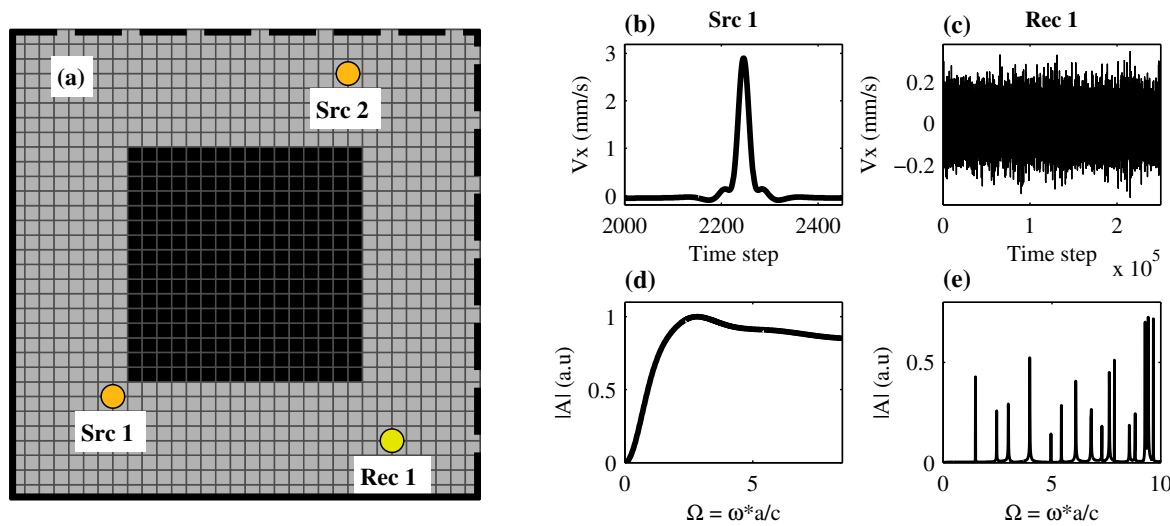

Figure 5.1: Input and output signals used for 2D time-domain simulations. The excitation signal is placed at two random and different locations. (a), (b) illustrate the Ricker wavelet used as excitation and the recorded signal at a random point in the unit cell, respectively. (c), (d) represent the Fourier transform of the previous mentioned signals. (e) show the location of both sources and detector in the unit cell.

A wide-band frequency signal is applied to excite all the modes needed to calculate the dispersion relation of the system. A ricker wavelet (the so-called Mexican hat signal) is applied as a prescribed displacement at a random point in the unit cell, as shown in Fig. 5.1(a), where a squared unit cell of $L=1 \mathrm{~m}$ is represented. The ricker wavelet is the second derivative of a gaussian pulse, 
defined as

$$
u(t)=a^{2}\left(a^{2} t^{2}-1\right) e^{-\frac{a^{2} t^{2}}{2}},
$$

where $f_{\max }=\frac{\sqrt{\pi} \cdot a}{2}$ is the central frequency of the spectrum. The main advantage of this signal is the absence of zero-frequency component, which is susceptible to introduce numerical artifacts in the scheme and should be filtered out from the recorded field displacement if present [Ward and Pendry, 1998]. However, due to the limited band-width of this signal, several ricker wavelets of different central frequencies are defined and added in time domain to cover a wide spectrum as illustrated in Figs. 5.1(b), 5.1(d), where the excitation signal is represented in time and frequency domains. Here, a combination of 4 ricker wavelets is designed to cover the desired frequency range (i.e., 12 first branches).

\section{Calculation of frequency band structure and Bloch mode shapes}

The procedure to obtain the band structure starts by setting up a value for the Bloch wave vector $\mathbf{k}$, which is inserted into the mass and stiffness matrices through the Bloch boundary transformation matrix (due to the dependence on the wave vector, the Bloch boundary transformation must be applied at each $\mathbf{k}$ point). Then, a transient excitation is applied at a random point within the unit cell for $t=0$. As time starts to evolve, Eqs. (5.14), (5.15) and (5.16) are used to solve for future displacements, velocities and accelerations at every node. A detector is randomly placed at one node in order to record the temporal signal for the displacement, as illustrated in Fig. 5.1(a). For a sufficiently large number of time iterations, the displacement field signal is long enough to capture all the resonant modes in the unit cell (see Section 5.4 for details). The time-history data is Fourier transformed into the frequency domain obtaining a set of resonant modes, as illustrated in Fig. 5.1(e). Each of the peaks of these resonant modes in the frequency domain constitute the eigenvalues of the vibrational modes for the given Bloch wave vector $\mathbf{k}$. The spanning of $\mathbf{k}$ along the IBZ allows for the obtention of the resonant modes, and hence, the dispersion relation.

In addition to the band structure calculation, the computation of Bloch mode shapes is also considered to fully characterize the response of the structure to dynamic loading. In opposition to the resolution of an eigenvalue problem, in which eigenvectors and eigenvalues result in the band structure 
and Bloch mode shapes, the proposed time-domain method allows for the obtention of the eigenvalues solely. However, the well-known experimental modal analysis can be considered to compute the Bloch mode shapes from the frequency response functions (FRF). Moreover, the Modal Assurance Criterion (MAC) [Allemang, 2003] is also employed in order to identify and solve degenerate modes at high symmetry points by comparing Bloch mode shapes obtained from sources placed at two different points in the unit cell (see Fig. 5.1(a)). The computation of Bloch mode shapes imply to record the displacement field signals at every node in order to obtain the FRF's. Hence, the number of detectors used is equivalent to the number of nodes in this case.

\subsection{Numerical examples}

The FETD method is used to compute the band structure of $1 \mathrm{D}$ and 2D phononic crystals in this section. Results are presented and compared to the solutions obtained by solving the standard eigenvalue problem by FE, selected as a reference method. A FDTD approach considering Bloch-Floquet boundary conditions is developed for the $1 \mathrm{D}$ case. All the numerical calculations are unit cells made of aluminum and ABS. The elastic properties of both materials are, density $\rho=2700 \frac{\mathrm{kg}}{\mathrm{m}^{3}}$, Young Modulus, $E=70 G P a$, Poisson coefficient, $\nu=0.34$ for Aluminum and $\rho=1050 \frac{\mathrm{kg}}{\mathrm{m}^{3}}, E=2.3 G P a$ and $\nu=0.34$ for ABS.

\section{D phononic crystal}

The one-dimensional case accounts for longitudinal wave propagation (axial modes) along a beam composed of ABS and aluminum with a filling fraction $f f=0.5$. Periodic boundary conditions (Bloch-Floquet) are applied on both ends of the beam. Hence, the beam constitutes a unit cell and represents a periodic medium of infinite length. Band structure results are depicted in Fig. 5.2, including calculations using FE (eigenvalue problem), FDTD and FETD. The unit cell is discretized into $n_{e l}=256$ elements and, for timedomain methods, the time evolution is simulated over $n_{s t}=2^{20}(1.048 .576)$ steps with a time interval $\Delta t=5.4 \times 10^{-7} \mathrm{~s}$, as stated in Eq.(5.17). As observed in Fig. 5.2 similar eigenvalues are obtained using FEM and FETD, and 


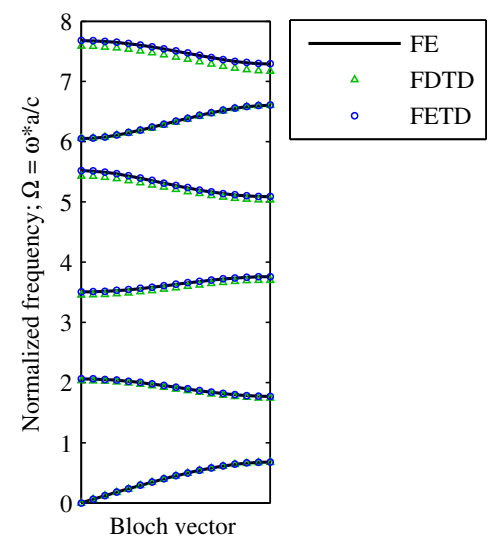

Figure 5.2: Phononic band structure for longitudinal elastic waves in a $1 \mathrm{D}$ unit cell. An aluminum and ABS matrix unit cell is studied, with a filling fraction, $f f=0.5$. The unitcell is discretized into $n_{e l}=256$ elements. Black solid lines, blue dots and green triangles correspond to FE, FETD and FDTD methods, respectively.

some discrepancies are found for FDTD case. These differences are analyzed in detail in Section 5.4.

\section{D phononic crystal}

The two-dimensional case consists of a square lattice composed of an ABS matrix and an aluminum solid inclusion in the center of the unit cell, as depicted in Fig. 5.1(a). The filling fraction of the periodic structure is $f f=0.25$. Longitudinal and shear wave propagation is considered. Figure 5.3 illustrates the FETD (blue/purple dots) and FEM (black solid lines) calculations of the band structure along the boundaries of the IBZ for (a) consistent and (b) lumped mass matrices. Both schemes assume a uniform grid of $n \times n=32^{2}$ two-node elements, resulting in $n_{\text {dof }}=2178$ total nodal degrees of freedom, which, after the application of Bloch BC's are reduced to $n_{\text {dof }}=2048$, since nodes lying at top and right sides of the unit cell belong to neighboring unit cells and are condensed out. The k-space is discretized such that $l_{k}=17$. Hence, a total of $n_{k}=49 \mathrm{k}$-points are evaluated to generate the band structure. The time interval is set to $\Delta t=1.57 \times 10^{-6} \mathrm{~s}$ and $n_{s t}=2^{18}(262.144)$ time steps. The corresponding frequency resolution is 
$\Delta f=1 /\left(\Delta t \cdot n_{\text {steps }}\right)=2.4 \mathrm{~Hz}$. An excellent agreement is noted between the proposed method and the standard eigenvalue problem in FEM. A detailed analysis of the accuracy in terms of the number of time steps and degrees of freedom and computational efficiency for $\mathbf{k}=(\pi / 2 a, \pi / 2 a)$ (grey dashed lines in Fig. 5.3(a)-(b)), is presented later in section V.

(a)

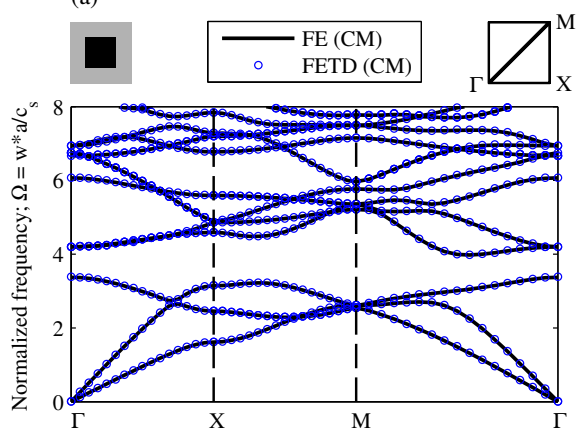

(b)

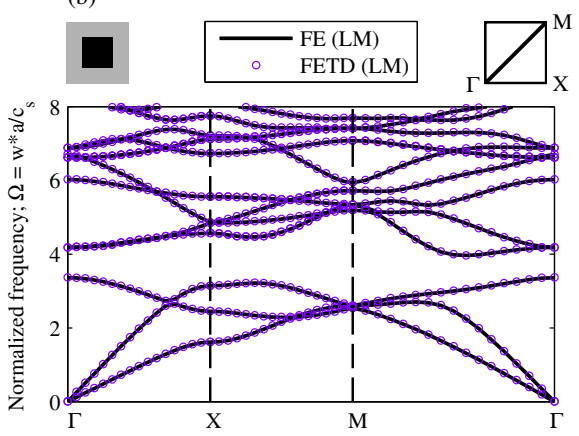

Figure 5.3: Phononic band structure for longitudinal and shear vertical elastic waves in a 2D square unit cell along the IBZ. FE and FETD methods for (a), consistent, (b) lumped mass cases are shown. Black solid lines and blue/purple dots corresponds to FE and FETD, respectively.

\section{Bloch mode shapes}

The periodic Bloch mode shapes computed with the time-domain algorithm, $\phi_{t d}$, are obtained from FRF's (experimental modal analysis) and expanded to full size using the transformation operation [Krattiger and Hussein, 2014]. A comparison of the Bloch mode shapes using FE and FETD for the $3^{r d}$ branch, $\mathbf{k}=(\pi / 2 a, \pi / 2 a)$, is depicted in Fig. 5.4 for consistent and lumped mass matrices, indicating an almost identical structure. The Bloch mode-shape error is quantified by comparing the FETD mode shapes, $\phi_{t d}$, and the FEM mode shapes, $\phi_{f e}$, and defined as

$$
e_{m}=1-\frac{a b s\left[\phi_{t d}{ }^{\prime} \phi_{f e}\right]}{\left|\phi_{t d}\right|\left|\phi_{f e}\right|}
$$

where $a b s[\cdot]$ denotes the complex modulus, and $|\cdot|$ denotes the vector twonorm. The resulting error is $e_{m, c m}=1.42 \cdot 10^{-4}, e_{m, l m}=4.66 \cdot 10^{-5}$ for the case shown in Fig. 5.3 corresponding to consistent and lumped mass matrices, 
respectively. It is also noted that the error is of the same order for the 10 first branches, below the following limits $e_{m, c m}<1.94 \cdot 10^{-4}, e_{m, l m}<1.56 \cdot 10^{-4}$.
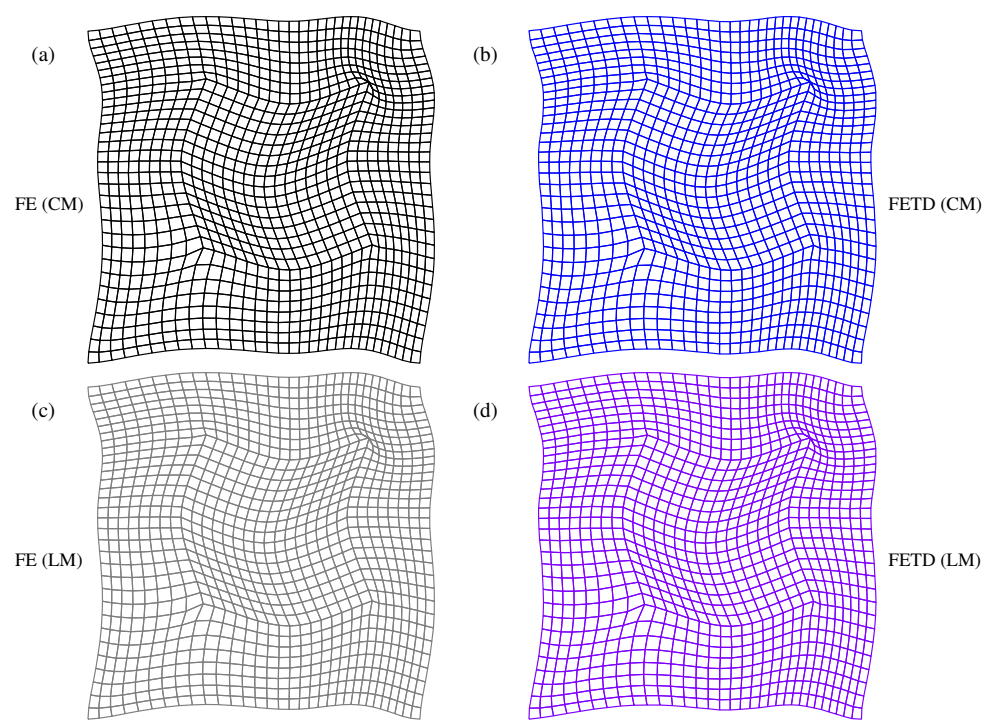

Figure 5.4: Mode shapes corresponding to branch $3, \mathbf{k}=(\pi / 2 a, \pi / 2 a)$ for the $2 \mathrm{D}$ phononic crystal. (a), (c) illustrate mode shapes extracted from FE calculations for consistent, and lumped mass, respectively. Bloch mode shapes calculated using FETD method are depicted for (c) consistent, and (d) lumped mass matrices.

\subsection{Method performance}

\section{Accuracy}

We evaluate the error on the total number of steps by comparing the eigenvalues using FETD to the ones obtained by FE for a unit cell of the same size $\left(n \times n=32^{2}\right)$, defined as

$$
e_{s t}=\frac{f_{t d}-f_{f e}}{f_{f e}} * 100,
$$

where $f_{t d}, f_{f e}$ are the computed eigenvalues for the $3^{r d}$ branch, $\mathbf{k}=\pi$ for time-domain methods, FDTD and FETD, and FE, respectively. 1D results are shown in Fig. 5.5(a). The error converges to a minimum and constant 

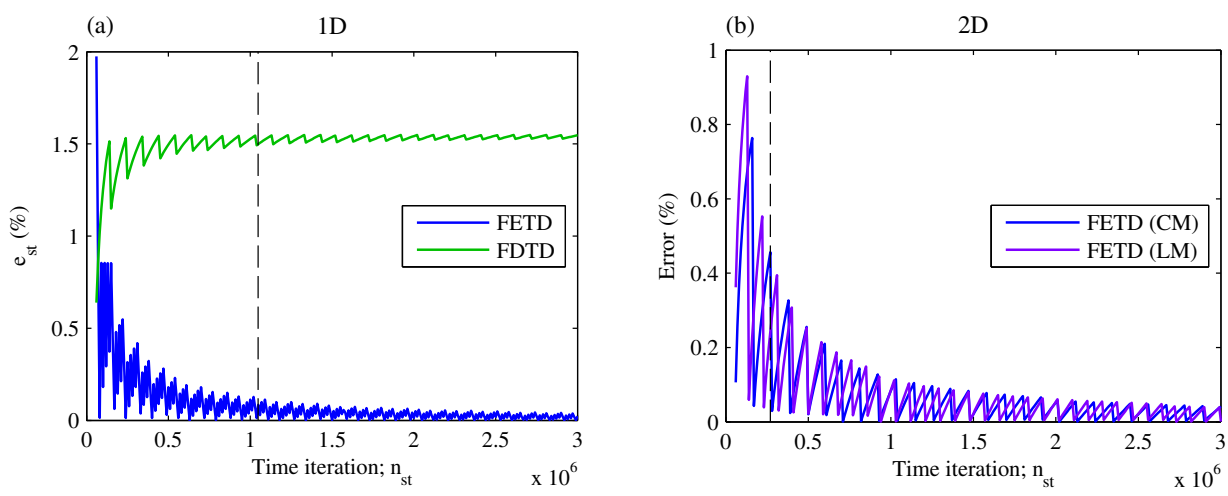

Figure 5.5: Error in frequency for increasing number of steps on time-domain methods for (a), $1 \mathrm{D}, \mathbf{k}=\pi / a$, and (b), $2 \mathrm{D}, \mathbf{k}=(\pi / 2 a, \pi / 2 a)$. Results are shown for branch 3. Vertical dashed black lines indicate the number of time iterations used in this work, $n_{s t, 1 D}=1.048 .576, n_{s t, 2 D}=262.144$.

value as the number of steps is increased, falling quickly below $1 \%$ and being almost negligible for $n_{s t}>2^{20}$. In contrast, the finite-difference approach converges to a slightly larger value of $1.5 \%$. Similar results are found in $2 \mathrm{D}$ for the comparison between consistent and lumped mass matrices, as shown in Fig. 5.5(b). As stated previously, the frequency resolution, $\Delta f$, is inversely proportional to the product of time interval and total number of steps, resulting in a higher resolution (lower $\Delta f$ ) as the number of steps is increased. This is clearly observed in the sawtooth pattern of the error signals, where $\Delta f$ is related to the difference between the local maxima and minima.

\section{Convergence}

The error in band structure calculations as a function of DOF is calculated using the following expression

$$
e_{d o f}=\frac{f_{N_{d o f, t d}}-f_{N_{r e f}, f e}}{f_{N_{r e f}, f e}} * 100,
$$

where $f_{N_{\text {dof,td }}}$ are the eigenvalues computed in time-domain for increasing number of DOF and $f_{N_{r e f}, f e}$ are eigenvalues computed with a FE model discretized into $n_{e l}=512$ and $n_{\text {dof }}=512$ periodic DOF in $1 \mathrm{D}$, and $n_{e l}=$ $128 * 128$ and $n_{\text {dof }}=32768$ periodic DOF in $2 \mathrm{D}$. The total number of set for these calculations is highlighted in Fig. 5.5 by vertical dashed lines. Error 

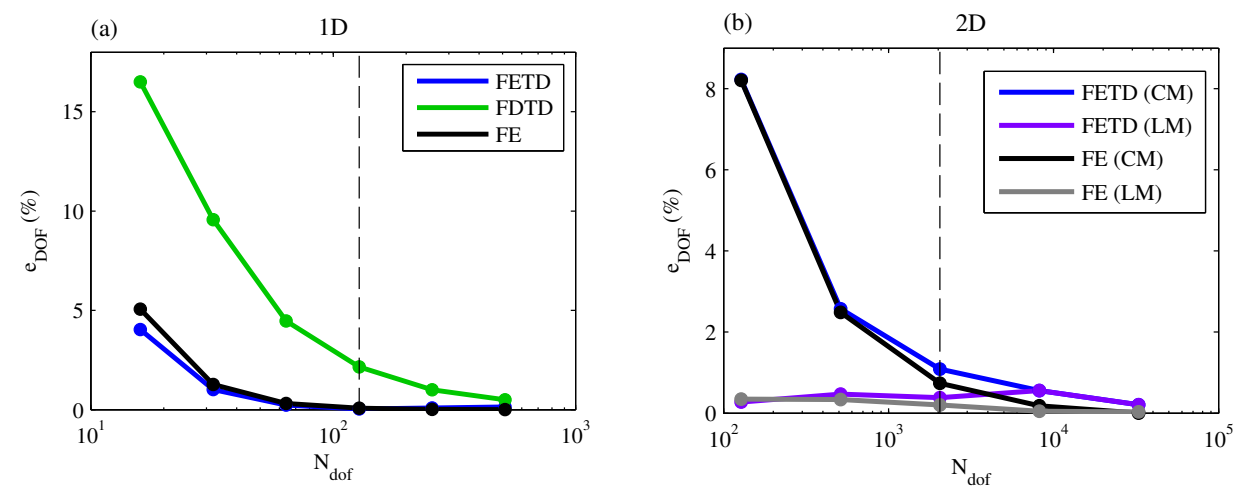

Figure 5.6: Error in frequency estimation for increasing number of DOF for (a), 1D, $\mathbf{k}=\pi$, and (b), $2 \mathrm{D}, \mathbf{k}=(\pi / 2 a, \pi / 2 a)$. Results are shown for branch 3 . Vertical dashed black lines illustrate the number of DOF used in Figs. 5.2, 5.3, $n_{d o f, 1 D}=256, n_{d o f, 2 D}=2048$.

results are depicted in Figs. 5.6(a)-(b). The convergence is slower for FDTD approach in $1 \mathrm{D}$, as for $\mathrm{FE}$ and FETD the error is reduced to values below $1 \%$ for a moderate number of DOF (32 in 1D, 2048 in 2D). It is noted that noticeable differences are not found when comparing FE and FETD methods, both for consistent and lumped mass matrices.

\section{Computational efficiency}

The computation time of the proposed method in time-domain depends mainly on the computational complexity of the matrix inversion needed to compute Eq. (5.16), which has to be done $n_{s t}$ times for each $\mathbf{k}$ point. On the other side, the computational complexity of the eigenvalue decomposition determines the computation time for FE approach. A similar trend is observed in Fig.5.7 for (a) 1D, and (b) 2D models, where computation time for models of increasing DOF is depicted in logarithmic scale. For low number of DOF eigenvalue decomposition is much more efficient. However, as the model is discretized into more elements, time-domain algorithms turn to be faster, as the computational complexity of the matrix inversion is slighlty lower than eigenvalue decomposition. It is noted that time-domain algorithms are strongly speeded-up when sparse matrices are used. Such a case is depicted in Fig.5.7(b) by comparing FETD results for consistent (blue line) and lumped mass (purple line) matrices. Moreover, the break-even point is found 
for $N_{D O F}=32768$ for the lumped mass case.
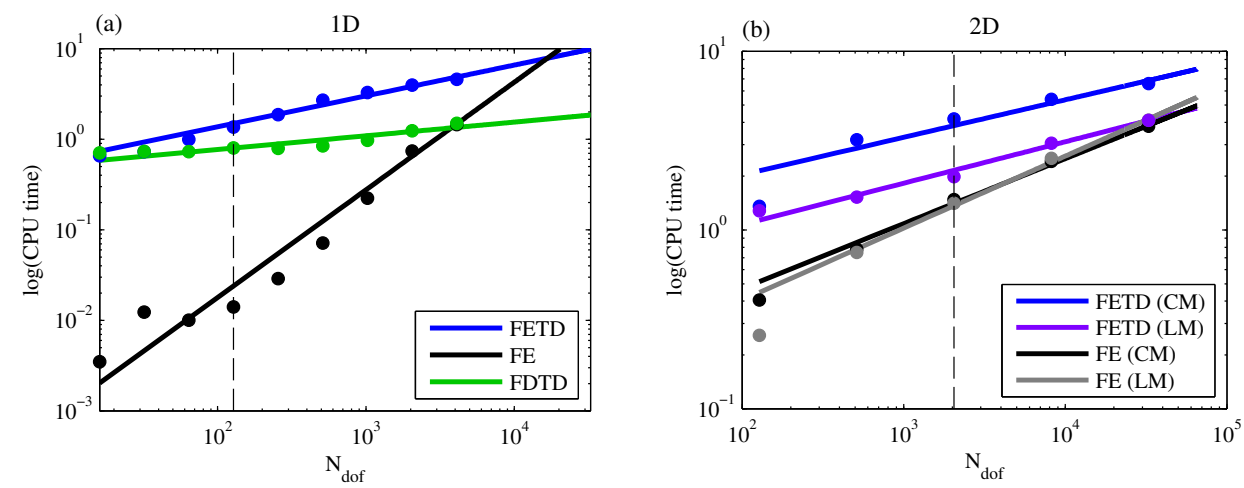

Figure 5.7: Computation time for increasing number of degrees of freedom for (a), 1D, $\mathbf{k}=\pi$, and (b), $2 \mathrm{D}, \mathbf{k}=(\pi / 2, \pi / 2)$. Results are shown for branch 3 . Vertical dashed black lines illustrate the number of DOFs used, $n_{\text {dof }, 1 D}=256, n_{\text {dof }, 2 D}=2048$.

A methodology for elastic band structure calculation in time-domain is presented in this chapter. The domain is discretized in space in finite elements and time using an explicit Newmark time-stepping scheme, and incorporates Bloch boundary conditions to reduce the model to a single unit cell. Band structure and modeshapes are calculated and compared to the results obtained using standard eigenvalue problem in FE for 1D and 2D cases, showing good accuracy and fast convergence. In 1D, band structure results are also compared with the finite difference approach showing faster convergence and lower error estimating eigenvalues regardless of the total number of steps. Ultimately, computation times are calculated for unit cells with increasing number of DOF showing a lower computational complexity for the time-domain approach in comparison to the resolution of an eigenvalue problem. Further conclusions will be presented in Chapter 6 .

\section{References}

Allemang, R. J. (2003). The modal assurance criterion-twenty years of use and abuse. Sound and vibration, 37(8):14-23.

Axmann, W. and Kuchment, P. (1999). An efficient finite element method for computing spectra of photonic and acoustic band-gap materials: I. scalar case. Journal of Computational Physics, 150(2):468-481. 
Busch, K., Von Freymann, G., Linden, S., Mingaleev, S., Tkeshelashvili, L., and Wegener, M. (2007). Periodic nanostructures for photonics. Physics reports, 444(3):101-202.

Cao, Y., Hou, Z., and Liu, Y. (2004). Finite difference time domain method for bandstructure calculations of two-dimensional phononic crystals. Solid state communications, 132(8):539-543.

Checoury, X. and Lourtioz, J.-M. (2006). Wavelet method for computing band diagrams of $2 \mathrm{~d}$ photonic crystals. Optics communications, 259(1):360-365.

Chiang, P.-j., Yu, C.-p., and Chang, H.-c. (2007). Analysis of two-dimensional photonic crystals using a multidomain pseudospectral method. Physical Review E, 75(2):026703.

Dobson, D. C. (1999). An efficient method for band structure calculations in $2 \mathrm{~d}$ photonic crystals. Journal of Computational Physics, 149(2):363-376.

Garcia-Pablos, D., Sigalas, M., De Espinosa, F. M., Torres, M., Kafesaki, M., and Garcia, N. (2000). Theory and experiments on elastic band gaps. Physical Review Letters, 84(19):4349.

Ho, K., Chan, C., and Soukoulis, C. (1990). Existence of a photonic gap in periodic dielectric structures. Physical Review Letters, 65(25):3152.

Hsieh, P.-F., Wu, T.-T., and Sun, J.-H. (2006). Three-dimensional phononic band gap calculations using the fdtd method and a pc cluster system. Ultrasonics, Ferroelectrics, and Frequency Control, IEEE Transactions on, 53(1):148-158.

Hussein, M. I. (2009). Reduced bloch mode expansion for periodic media band structure calculations. In Proceedings of the Royal Society of London A: Mathematical, Physical and Engineering Sciences, volume 465, pages 2825-2848. The Royal Society.

Hussein, M. I., Leamy, M. J., and Ruzzene, M. (2014). Dynamics of phononic materials and structures: Historical origins, recent progress, and future outlook. Applied Mechanics Reviews, 66(4):040802.

Johnson, S. and Joannopoulos, J. (2001). Block-iterative frequency-domain methods for maxwell's equations in a planewave basis. Optics Express, 8(3):173-190.

Jun, S., Cho, Y.-S., and Im, S. (2003). Moving least-square method for the band-structure calculation of $2 \mathrm{~d}$ photonic crystals. Optics express, 11(6):541-551.

Kafesaki, M. and Economou, E. N. (1999). Multiple-scattering theory for three-dimensional periodic acoustic composites. Physical review B, 60(17):11993.

Kafesaki, M., Penciu, R., and Economou, E. (2000). Air bubbles in water: a strongly multiple scattering medium for acoustic waves. Physical review letters, 84(26):6050.

Kittel, C. and Holcomb, D. F. (1967). Introduction to solid state physics. American Journal of Physics, 35(6):547-548.

Krattiger, D. and Hussein, M. I. (2014). Bloch mode synthesis: Ultrafast methodology for elastic band-structure calculations. Physical Review E, 90(6):063306.

Kushwaha, M. S., Halevi, P., Dobrzynski, L., and Djafari-Rouhani, B. (1993). Acoustic band structure of periodic elastic composites. Physical Review Letters, 71(13):2022.

Leung, K. and Qiu, Y. (1993). Multiple-scattering calculation of the two-dimensional photonic band structure. Physical Review B, 48(11):7767. 
Liu, Z., Chan, C., Sheng, P., Goertzen, A., and Page, J. (2000). Elastic wave scattering by periodic structures of spherical objects: Theory and experiment. Physical Review B, 62(4):2446.

Meade, R. D., Rappe, A., Brommer, K., Joannopoulos, J., and Alerhand, O. (1993). Accurate theoretical analysis of photonic band-gap materials. Physical Review B, 48(11):8434.

Moreno, E., Erni, D., and Hafner, C. (2002). Band structure computations of metallic photonic crystals with the multiple multipole method. Physical Review B, 65(15):155120.

Newmark, N. M. (1959). A method of computation for structural dynamics. Journal of the Engineering Mechanics Division, 85(3):67-94.

Pask, J., Klein, B., Sterne, P., and Fong, C. (2001). Finite-element methods in electronicstructure theory. Computer Physics Communications, 135(1):1-34.

Pendry, J. and MacKinnon, A. (1992). Calculation of photon dispersion relations. Physical Review Letters, 69(19):2772.

Qiu, M. and He, S. (2000). A nonorthogonal finite-difference time-domain method for computing the band structure of a two-dimensional photonic crystal with dielectric and metallic inclusions. Journal of applied physics, 87(12):8268-8275.

Robertson, W. and Rudy III, J. (1998). Measurement of acoustic stop bands in twodimensional periodic scattering arrays. The Journal of the Acoustical Society of America, 104(2):694-699.

Rubio, C., Caballero, D., Sánchez-Pérez, J., Martinez-Sala, R., Sánchez-Dehesa, J., Meseguer, F., and Cervera, F. (1999). The existence of full gaps and deaf bands in twodimensional sonic crystals. Journal of lightwave technology, 17(11):2202.

Sigalas, M. and Economou, E. (1993). Band structure of elastic waves in two dimensional systems. Solid State Communications, 86(3):141-143.

Tanaka, Y., Tomoyasu, Y., and Tamura, S.-i. (2000). Band structure of acoustic waves in phononic lattices: Two-dimensional composites with large acoustic mismatch. Physical Review B, 62(11):7387.

Wang, X., Zhang, X.-G., Yu, Q., and Harmon, B. (1993). Multiple-scattering theory for electromagnetic waves. Physical Review B, 47(8):4161.

Ward, A. and Pendry, J. (1998). Calculating photonic greens functions using a nonorthogonal finite-difference time-domain method. Physical Review B, 58(11):7252.

Yan, Z.-Z. and Wang, Y.-S. (2006). Wavelet-based method for calculating elastic band gaps of two-dimensional phononic crystals. Physical Review B, 74(22):224303.

Yang, H. Y. D. (1996). Finite difference analysis of 2-d photonic crystals. Microwave Theory and Techniques, IEEE Transactions on, 44(12):2688-2695. 


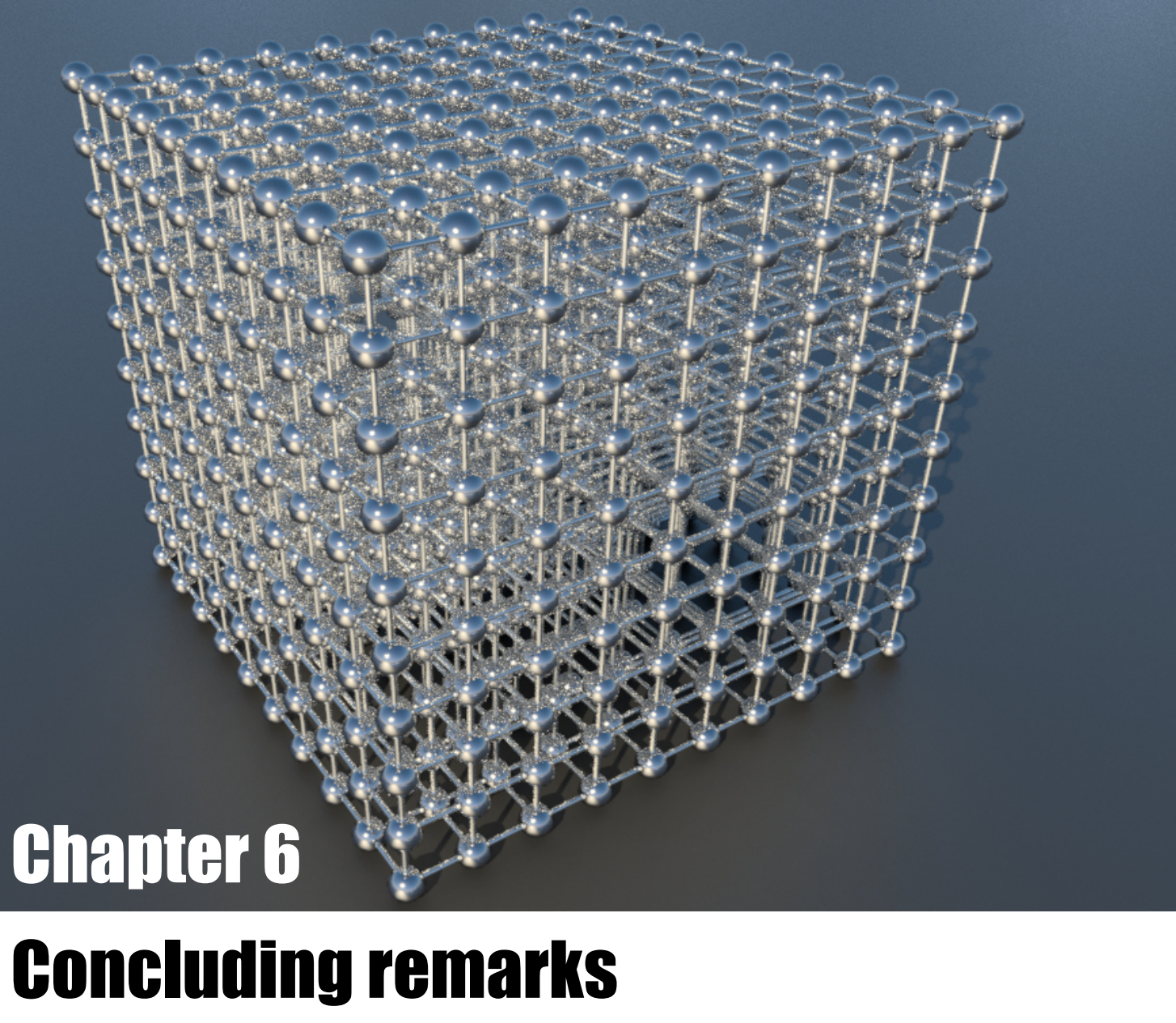

\subsection{Conclusions}

The work reported in this thesis can be divided in two different parts. On the first part we concentrated in the study of transmission, reflection and absorption of acoustic waves in phononic crystals, considering wave focusing and collimation in transmission in Chapter 2, field enhancement due to soft reflection in chirped structures in Chapter 3, and the control of losses in layered dissipative systems in Chapter 4 . The second part of the study is presented in Chapter 5 and was devoted to the development of a computational methodology for elastic band structure calculation in the framework of the finite element in time-domain method. We present here conclusions on the topics considered in this work and future lines of research as well potential technological applications. 


\subsubsection{Control of wave propagation in transmission}

In Chapter 2 we illustrated the concept of focalization and collimation of beams in terms of the relation between the curvature of the spatial dispersion curves of the sonic crystal and the angular components of the incident beam, and we performed numerical simulations in a simple 2D geometry to explain the concept. In general terms, for a finite size sonic crystal, the focalization of waves behind the crystal is related to the negative diffraction experienced by the wave inside the structure. In this situation, a wave front acquires positive curvature inside the periodic structure due to negative diffraction, when the wave front go through the crystal and propagates in the homogeneous medium with positive diffraction, the accumulated phase differences are compensated at a given distance where the beam is focused. However, the focusing mechanism is strongly influenced by the angular spectrum of the incident beam which in turn depends on the size of the source, a narrower source posses a broader angular spectrum and viceversa. It was observed that the interplay between the angular spectrum of the source and the spatial dispersion curves of the crystal determines the beam quality and amplitude of the focused beam. Considering the optimum case, where the main angular components of the source fitted completely with the corresponding isofrequency contour at a given frequency, a well focused beam was obtained and the focusing distance predicted from the curvature of the spatial dispersion curves of the crystal. Moreover, the focusing distance depends on frequency, adding an interest feature to the focusing mechanism, as slight variations in the frequency of the incident beam result in a considerable variation in the focusing distance.

A two-dimensional case was considered in this part of the chapter for convenience, allowing the use of sources of different sizes. However, similar results were obtained for a 3D wood-pile sonic crystal and an acoustic source that possess radial symmetry (see Appendix A.1). However, the strong scattering inside the sonic crystal in the diffractive regime and the absence of symmetry between the source and the sonic crystal reduces the efficiency of the focusing system in terms of gain. In the second part of Chapter 2 we presented a different approach to increase the efficiency of the focusing system. With this regard, an axisymmetric structure composed of rigid toroidal scatterers that match the symmetry of the source and working in the long-wavelength regime was designed, as a gradient index lens. Using the homogenization theory, that allow to consider the structure as an homogeneous medium with 
effective properties, we considered a hiperbolic secant profile to define the refractive index variation in the radial direction. We compared the results from the ray tracing trajectories, the effective fluid medium approximation and numerical simulations using the axisymmetric structure, all in good agreement. Finally, the efficiency in terms of gain of the proposed system was also validated numerically, showing a remarkable value of sound amplification higher than $8 \mathrm{~dB}$ was obtained, which is also in agreement with the experimental results shown in Appendix A.2.

\subsubsection{Soft reflection in chirped sonic crystals}

Chapter 3 was devoted to the study of reflection phenomena in chirped sonic crystals. We demonstrated a mechanism for sound field enhancement for plane waves propagating along chipred sonic crystals in which the lattice constant is reduced along the propagation direction. This mechanism is related to the soft reflection of waves due to a progressive slowing down of the sound velocity as it propagates through the crystal until it reaches a local band gap, and occurs for frequencies around the first band gap. In this situation, the acoustic wave energy is selectively concentrated at different planes of the crystal depending on the parameters of the structure and the frequency. It is concluded that the theoretical approach based in a coupled mode theory predicts adequately the sound field enhancement mechanism for different chirped profiles. The results and insights from the CMT, as well as the numerical harmonic analysis and experimental results confirm the validity of the main assumption adopted, the consideration of our structure as locally periodic. This is also validated by means of the analysis in time-domain, that allow to measure the time spreading of the reflected pulse and compare this results to the ones extracted from the analysis of the band structure, showing a good agreement.

\subsubsection{Absorption in periodic layered media}

In Chapter 4 an anomalous phenomena related to periodic structures formed by absorptive materials is described and measured experimentally in the context of sound waves. The study indicates an enhancement and reduction of transmission and absorption at frequencies around the band gaps. The effect is described in a general context (for different types of waves) by the disipative CMT equations, calculating the transmission, reflection and absorption coefficients, and showing the differences encountered when comparing to the 
well known reflection and transmission from conservative periodic structures. The experimental study is particularized to the case of sound waves, demonstrating a simultaneous enhancement of reflection and transmission as well as a reduction in the absorption at frequencies around the band gap in good agreement the TMM predictions.

The concept of enhanced absorption in porous lamella crystals is also demonstrated. A modified TFM is firstly verified by comparison to the standard TFM in the impedance tube, and then applied to measure a system made out of lossy lamellas mounted onto a rigid backing in the anechoic chamber, demonstrating the ability to increase absorption by lowering the effective amount of material, not only for normal incident radiation but for all-angle incidence, and for a broadband frequency range. Applying this concept, highly absorptive materials can be engineered by means of increasing the sound material interaction strength (i.e., the group time delay) resulting in a dramatic decrease of reflectance. The counter intuitive ability to increase absorption by lowering the effective ammount of material provides an interesting feature for minimizing noise in different technological applications by producing an efficient acoustic sealing.

\subsubsection{Band structure calculations in time-domain}

A new methodology for elastic band structure calculation in time-domain was presented in chapter 5 . Band structure and modeshapes were calculated and evaluated in terms of accuracy and convergence, using the standard eigenvalue problem in $\mathrm{FE}$ as the reference. In terms of computational efficiency, when a high number of DOF is needed to discretize the unit cell, the computational complexity of the eigenvalue decomposition turns into higher computation times than the proposed approach in time-domain. In conclusion, a variety of studies making use of complicated geometries or even the introduction of time-dependent designs, (ie., unit-cells incorporating fluid flow channels, etc.) can benefit from this methodology.

On the other hand, further improvements can be implemented in order to increase the computational efficiency while maintaining similar accuracy. A pseudperiodic initial field distribution, consistent with the periodicity enforced at the boundaries of the unit cell, can reduce considerably the total number of time steps needed to compute the band structure [Cao et al., 2004]. Moreover, the length of the time-history data can be dramatically reduced 
considering a post-processing method for the transformation to the frequency domain different from the fourier transformation, the high-resolution spectral estimation method [Su and Wang, 2010].

\subsection{Future work}

Focalization and collimation of acoustic waves is a topic that has received a lot of attention over the years and the time to move to technological applications is arriving. With this regard an interesting application in the audible regime is found in Ref. [R, 2009] in the context of phase control in line array waveguides. In this work, the use of multiple scatterers with lenticular shape is proposed. These scatterers ara arranged periodically to form a triangular shaped structure an placed inside a horn aiming to equal the pase at the output of the horn. We are interested in an improvement of this design using the concepts presented in this work under two different approaches, the design of a structure to work in the long wavelength regime and the improvement of the shape, size and distribution of the scatterers using optimization techniques.

In the field of acoustics and considering conservative systems, the results are independent of the spatial scale of the structure, and in principle, the soft reflection phenomenon could be scaled-down and observed in micro- or nanoscale phononic (so called hypersonic) crystals [Gorishnyy et al., 2005]. At these scales, sound waves are described in terms of phonons, and the ideas presented in this work could find application for heat management in acoustical or acousto-optical devices. Recent works in this direction show indeed that manipulation of phonon dispersion properties can allow thermal transport control [Hopkins et al., 2010]. Generally, the effect of wave energy concentration demonstrated here opens a possibility of increasing the efficiency of detectors and absorbers, both in acoustics and optics, since slow phonons and photons can be absorbed and harvested with a higher probability.

An interesting effect observed during the realization of this work was the appearence of an intense field enhancement in chirped structures at very high frequencies that cannot be explained using the coupled mode theory. This enhancement seems to be of a different nature than the one reported in this work and could be explained in terms of resonances or defect modes. We will explore this effect and try to find the physical principle.

Dissipation in periodic structures is probably the topic less explored so far 
and it is now a hot topic in the phononics community. The concept of critical coupling to achieve maximum absorption is clearly a valuable line of research to explore [Romero-García et al., 2015] in the pursuit of a real deaf body, i.e., a system capable of a broadband absorption and minimum reflection.

In the context of band structure calculations, we have succesfully applied the methodology presented in this work to the computation of the band structure in phononic crystals and metamaterials incorporating an embedded network of fluidic channels, where the resolution of an eigenvalue problem in finite element is not suitable to compute the band structure. In this work, presented in Phononics 2015: 3rd International Conference on Phononic Crystals/Metamaterials, Phonon Transport and Phonon Coupling, under the title: An elastic medium with a time-changing band structure, we demonstrated how the band structure of these structures can be tuned by incorporating fluidic channels. For this system, we utilized and integrated computational methodlogy based on a finite-element time-domain scheme, channel fluid dynamics and fluid-structure interaction, and demonstrated how the band structure is tuned "on the fly" by varying the composition of the fluid. However, in this work we assumed an incompressible fluid flow at low Reynolds numbers for the fluid channels. The next step in this research line is to implement the full Navier-Stokes equations in order to increase the influence of the fluid properties in the band structure.

\section{References}

Cao, Y., Hou, Z., and Liu, Y. (2004). Finite difference time domain method for bandstructure calculations of two-dimensional phononic crystals. Solid state communications, 132(8):539-543.

Gorishnyy, T., Ullal, C. K., Maldovan, M., Fytas, G., and Thomas, E. L. (2005). Hypersonic phononic crystals. Phys. Rev. Lett., 94:115501.

Hopkins, P. E., Reinke, C. M., Su, M. F., Olsson III, R. H., Shaner, E. A., Leseman, Z. C., Serrano, J. R., Phinney, L. M., and El-Kady, I. (2010). Reduction in the thermal conductivity of single crystalline silicon by phononic crystal patterning. Nano letters, 11(1):107-112.

R, M. (2009). Guía acústica de onda y sistema electroacústico que comprende dicha guía de onda.

Romero-García, V., Theocharis, G., Richoux, O., Merkel, A., and Pagneux, V. (2015). Perfect and broadband acousticabsorption by critically coupledsub-wavelength resonators. $\mathrm{Su}, \mathrm{X} .-\mathrm{X}$. and Wang, Y.-S. (2010). Calculation of two-dimensional phononic energy bands by using fdtd and high-resolution spectral estimation. In PROCEEDINGS OF THE 2ND 
INTERNATIONAL SYMPOSIUM ON COMPUTATIONAL MECHANICS AND THE 12TH INTERNATIONAL CONFERENCE ON THE ENHANCEMENT AND PROMOTION OF COMPUTATIONAL METHODS IN ENGINEERING AND SCIENCE, volume 1233, pages 1524-1528. AIP Publishing. 



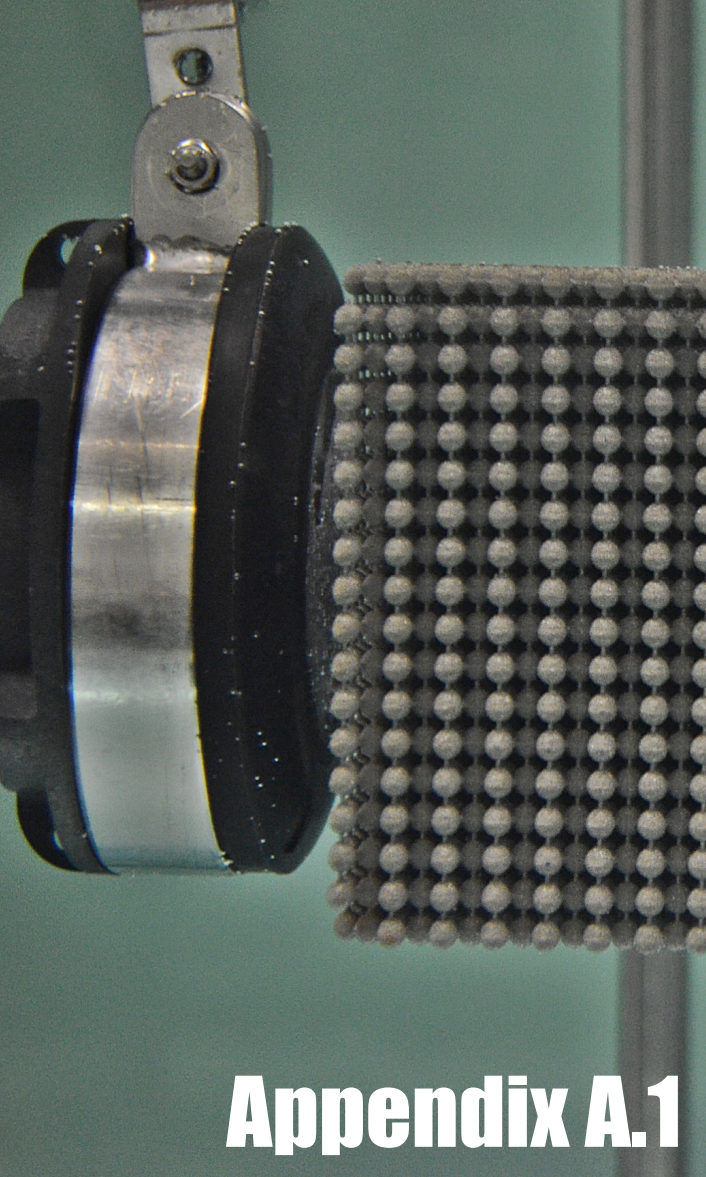

\section{Formation of Collimated Sound Beams in 3D Sonic Grystals}

A theoretical and experimental study of the propagation of sound beams inside and behind three-dimensional sonic crystals at frequencies close to the band edge is presented in this paper. Focusing and collimation of the beam behind the crystal is predicted theoretically and numerically and demonstrated through experimental measurements. The presented effects are analyzed and interpreted in terms of the curvature of spatial dispersion curves and surfaces of the sonic crystal and are related to the negative diffraction close to the edge of the propagation band. The author of this thesis participated in the numerical simulations and carried out the experimental measurements and post-processing of these results. 


\title{
Formation of collimated sound beams by three-dimensional sonic crystals
}

\author{
A. Cebrecos, ${ }^{1, a)}$ V. Romero-García, ${ }^{1}$ R. Picó, ${ }^{1}$ I. Pérez-Arjona, ${ }^{1}$ V. Espinosa, ${ }^{1}$ V. J. Sánchez-Morcillo, ${ }^{1}$ and K. \\ Staliunas ${ }^{2}$ \\ ${ }^{1)}$ Instituto de Investigación para la Gestión Integrada de Zonas Costeras, Universidad Politécnica de Valencia (UPV), \\ Paranimf 1, 46730, Grao de Gandia, Spain \\ ${ }^{2)}$ ICREA, Departament de Física i Enginyeria Nuclear, Universitat Politècnica de Catalunya, Colom, 11, E-08222, \\ Terrasa, Barcelona, Spain
}

(Dated: 8 February 2012)

\begin{abstract}
A theoretical and experimental study of the propagation of sound beams in- and behind three-dimensional sonic crystals at frequencies close to the band edges is presented. An efficient collimation of the beam behind the crystal is predicted and experimentally demonstrated. This effect could allow the design of sources of high spatial quality sound beams.
\end{abstract}

PACS numbers: 43.35.-c

Focusing and propagation of sound beams is of fundamental importance in several branches of applied acoustics, such as tomography, acoustic microscopy and imaging or sonar communication. To achieve optimal focusing, and to maximize spatial quality of the sound beams, several mechanisms have been proposed in acoustics, like the use of acoustic lenses ${ }^{1}$ or the design of Gaussian beam transducers. ${ }^{2}$ Recently it has become apparent that the materials whose properties are modulated in space, also known as sonic crystals (SCs) in acoustics ${ }^{3}$ or photonic crystals in optics ${ }^{4}$, can modify the spatial dispersion of propagating waves. This feature opens new possibilities to control the diffractive broadening of sound beams. In particular the beams can propagate in modulated material without diffraction (the effect also referred to as selfcollimation), as predicted and demonstrated in optics ${ }^{5}$ and in acoustics. ${ }^{6}$ Self-collimation is based on the existence of flat segments of spatial dispersion curves (the curves of constant frequency in $\vec{k}$-space). More recently the three-dimensional (3D) self-collimation by SCs was experimentally demonstrated, ${ }^{7}$ which is based on the formation of flat areas of the isofrequency surfaces.

In addition to non-diffractive propagation inside the SCs, the modification of the spatial dispersion can also produce phenomena outside the crystal such as lensing ${ }^{8,9}$ and superlensing. ${ }^{10}$ These beam propagation effects behind the SCs are related with the negative diffraction inside the periodic structure. The character of the beam propagation behind the SC depends on the wave front of the beam acquired in the system. In particular, if the wave front of the beam acquires positive curvature (due to propagation in a material with negative, or anomalous diffraction), the beam can be focused behind the modulated medium, which enables above discussed lensing and superlensing effects.

Although the focusing of sound beams behind a 2D SCs is being intensively investigated ${ }^{11}$ the overall picture of the beam formation and propagation is still unclear.

\footnotetext{
a) Electronic mail: alcebrui@epsg.upv.es
}
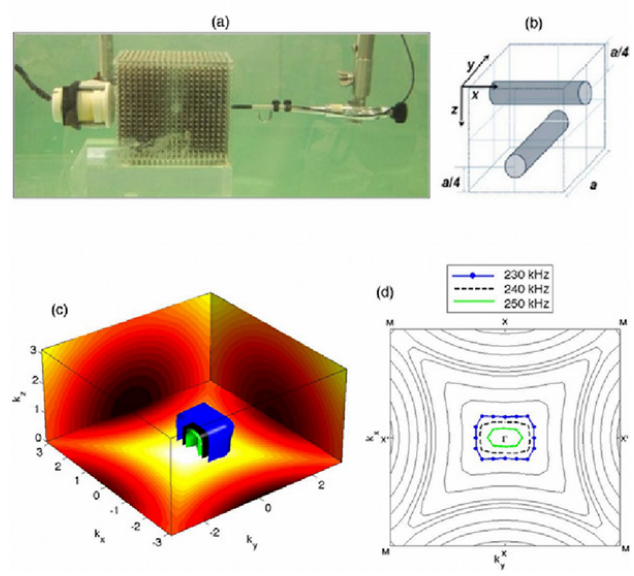

FIG. 1. (Color online) (a) Experimental configuration, (b) unit cell of the 3D SC, (c) isofrequency surfaces and (d) crosssection of dispersion surfaces.

Apart from the above mentioned phase transformation effects due to the negative diffraction of waves propagating inside of the SC, spatial (or angular) filtering effects also come into play. The negatively curved segments of dispersion lines are generally surrounded by the angular bandgaps, which are angular areas where sound cannot propagate. The latter results in a modification of the angular spectrum of the beams, ${ }^{12}$ recently demonstrated in both optics ${ }^{13}$ and acoustics. ${ }^{14}$ These two beam formation mechanisms combine, and give rich possibilities of formation of the beams with desired spatial characteristics (angular distributions) and with desired character of focusing.

In the present work we study, experimentally and theoretically, the sound beam formation behind a 3D SC with a woodpile-like structure. We experimentally demonstrate the formation of high spatial quality and wellcollimated beams behind the SC, based on the above 
described spatial filtering and negative diffraction effects. In this letter, we present first the isofrequency contours of $\mathrm{SC}$, and identify the ranges of the frequencies where the curvature of the isofrequency surfaces is positive. Based on these results we design the samples, and perform the sound beam propagation experiments. The most important result is the experimental demonstration of the formation of a well focused beam. Finally, we investigate the beam propagation in a simplified paraxial approximation, and obtained a good quantitative interpretation of the experimental measurements.

Figure 1a shows the experimental setup. The 3D SC is formed by two $2 \mathrm{D}$ structures of square symmetry, embedded one into another after a relative rotation by 90 degrees, which results in a $3 \mathrm{D}$ woodpile-like structure. ${ }^{7}$ Each of $2 \mathrm{D}$ structures consist of $20 \times 20$ matrix of steel cylinders of a radius $r=0.8 \mathrm{~mm}$, and the lattice constant $a=5.25 \mathrm{~mm}$ (see unit cell in Fig. 1b). The beam, emitted by an ultrasonic source, propagates through the SC along the $z$ direction. The acoustic field is measured by a needle hydrophone positioned by a three motorized axes governed by acquisition system. As shown in Fig. 1a, the experimental set up is immersed in a plexiglass tank filled with distilled water.

The eigenfrequency analysis of the sound wave propagation was performed numerically using Finite Element Method. ${ }^{15}$ The periodicity of the system is considered by imposing Bloch-Floquet boundary conditions of the unit cell (Fig. 1b). The path around the first irreducible Brillouin zone represents the main directions of symmetry in $3 \mathrm{D}$. We analyze the propagation along $\Gamma \mathrm{X}$ direction in the present work.

Figure 1c shows the isofrequency surfaces for three different frequencies $(230,240$ and $250 \mathrm{kHz})$ in the second band as well as the cross sections of the isofrequency surfaces by $k_{z}=0, k_{x}=0$ and $k_{y}=0$ planes respectively. Fig. 1c shows the quarter of the isofrequency "bubble" for these three frequencies. The isofrequency lines in $k_{z}=0$, plane are shown in detail in Fig. 1d. The lowest of highlighted frequencies $(230 \mathrm{kHz})$ corresponds to non-diffractive propagation inside the SC (flat isofrequency line). The isofrequency surfaces (and the lines in the cross plane) at slightly higher frequencies have areas with a positive curvature, which cause the desired focusing behaviour.

The experimental measurements of the beams propagating behind the SC are summarized in Fig. 2. Three different frequencies are represented in (a) upper, (b) middle, and (c) bottom panels. The upper panel (235 $\mathrm{KHz}$ ) shows the beam propagation for frequency corresponding to self-collimation inside the crystal. ${ }^{7}$ The bottom panels in Fig. $2(260 \mathrm{kHz})$ show the beam propagation for the case when a strongly curved and relatively small "bubble" of isofrequency surface occurs (Fig. 1c). As the area of the isofrequency surface responsible for the negative diffraction and eventually for focusing is very small, just the central (paraxial) part of the angular spectrum is focalized. One part of the remaining
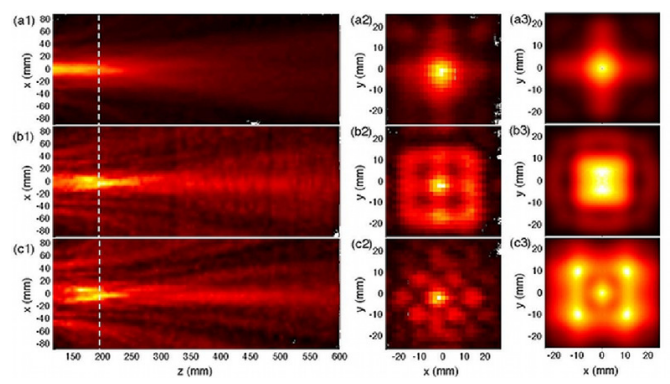

FIG. 2. (Color online) Experimental measurements and simulations of the acoustic field pattern behind the SC at (a) 235 $\mathrm{kHz}$, (b) $250 \mathrm{kHz}$ and (c) $260 \mathrm{kHz}$. (1) XZ-cross-section of the beam behind the SC; experimental (2) and numerical (3) ultrasound field distribution in the XY plane at point $z_{1}=195$ $\mathrm{mm}$ behind the SC.

angular components is reflected, as it corresponds to the angular bandgaps. The other part of angular components propagates along different directions, giving rise to side-lobes as seen in Fig. 2c. The intermediate situation, corresponding to the frequency $250 \mathrm{kHz}$, is shown in the middle panels of Fig. 2. The diffraction is negative for the propagation inside the SC due to positive curvature of the dispersion curves (see Figs. 1c and 1d). The isofrequency "bubble" is large enough to transmit a sensible portion of the angular spectrum. This case is most relevant for the goals of this work.

We interpret the focusing of the beam in the terms of Ref. 11: the sound beam propagating in bulk of SC with negative diffraction accumulates the increasing positive (anomalous) curvature of the beam wave front. Behind the SC, the propagation in the (normally diffracting) homogeneous medium compensates the accumulated negative diffraction acquired inside the SC. The beam is focalized at some distance $z_{f}$ where the negative diffraction inside the SC and positive diffraction behind the SC compensate one another.

The analytical estimation of the focal distance is possible considering the approximation of small filling fraction of the SC, $f=V_{s} / V_{u c}$ ( $V_{s}$ and $V_{u c}$ are the volume occupied by the scatterer and the unit cell respectively). In this approximation the diffraction coefficient (i.e. the curvature of the spatial dispersion curve, and/or surface) can be analytically calculated. ${ }^{11}$ Following the above interpretation, the negative diffraction of the $\mathrm{SC}$ is compensated at a distance $z_{f}$ behind the SC (measured from the input plane of the SC):

$$
z_{f}=L \frac{f^{2}}{\Delta \Omega^{3}}
$$

where $L$ is the length of the SC, and $\Delta \Omega=\left(\Omega_{g}-\Omega\right) / \Omega_{g}$ with $\Omega_{g}=\omega_{g} a / 2 \pi c_{h}$ being the normalized Bragg frequency and $c_{h}$ the speed of sound of the host medium, i.e. in water. 

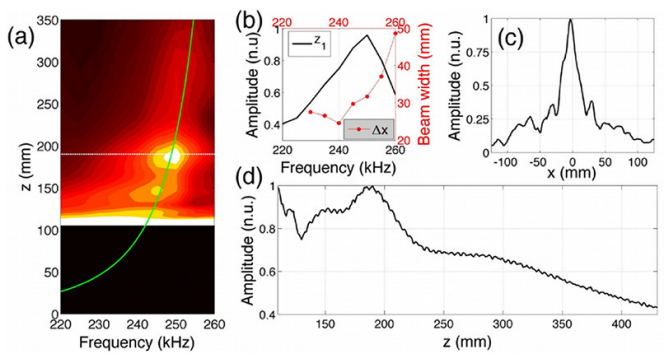

FIG. 3. (Color online) (a) Density plot representing the measurements of the on-axis intensity distribution on the frequency-z plane (distances measured from the transducer). Continuous green line corresponds to analytical fit (see text). Black area represents the space occupied by the SC. The white dashed line represents the point $z_{1}$ (b) Continuous line represents the measured beam amplitude in normalized units at point $z_{1}$ and red dotted line represents the measured beam width. (c) Experimental profiles in the $x$-axis of the beam at $z_{1}$ point and (d) measured amplitude in normalized units on the $z$-axis at $250 \mathrm{kHz}$.

The experimental results were compared with the analytical study of beam focusing, as shown in Fig. 3. In Fig. 3a the absolute value of the intensity behind the crystal on the $z$-axis is mapped depending on the frequency. Green continuous line represents the analytical fit of the focal distance calculated from Eq. (1) considering $L=20 a, \Omega_{g}=1$, and $f=0.05$. The parameter $f$ is a fit parameter. We notice that due to the fact that Eq. 1 has been obtained for 2D structures with low filling fraction, we use a fit parameter to take this into account. For this case, the frequency of zero diffraction point or self collimation corresponds to $\Omega_{Z D P}=\left(1-f^{2 / 3}\right) \Omega_{g}$, $\left(\nu_{Z D P}=238 \mathrm{kHz}\right)$.

The focusing for a frequency range around the optimal one is evidenced in Figs.3b-d. Fig.3b shows both the amplitude at point $z_{1}$ (black continuous line) and the beam width (red dots) depending on the frequency. Figs. $3 \mathrm{c}$ and $3 \mathrm{~d}$ show the profile at $z_{1}$ along the $x$-axis and the transversal cross-section along the $z$ direction in $x=0$ for the focusing frequency $250 \mathrm{kHz}$ respectively.

In summary, we have experimentally demonstrated the collimation of the beams behind a 3D SC. The obtained results are interpreted and analyzed in terms of curvatures of spatial dispersion curves and surfaces of the SC, and rely on the negative diffraction close to the edge of the propagating band. The experimental results fit well with the numerical simulations as well as with analytical predictions in Ref. 11. The tunability of the focal distance has been also demonstrated, showing that the beam intensity in the focus as well as the broadening of the beam along the propagation depends on the frequency, which give additional options for applications.

The overall focusing process is interpreted in terms of the interplay between two related but different effects: the focusing of the beam, due to curvature of spatial dispersion curves; and the spatial filtering effect, due to the size of the isofrequency "bubble". The optimum result comes from a compromise between these two ingredients.

\section{ACKNOWLEDGMENTS}

The work was financially supported by Spanish Ministry of Science and Innovation and by European Union FEDER through projects FIS2008-06024-C02-02,-03 and MAT2009-09438. V.R.G. is grateful for the support of post-doctoral contracts of the UPV CEI-01-11. K.S. acknowledges the grant of UPV PAID-02-01.

${ }^{1}$ T.W. Shield, and J.G. Harris, J. Acoust. Soc. Am. 75, 1634 (1984).

${ }^{2}$ D. Huang and M. Breazale, IEEE Transactions on Ultraonics, Ferroelectrics and Frequency Control 53, 1018 (2006).

${ }^{3}$ J. V. Sánchez-Pérez, D. Caballero, R. Martínez-Sala, C. Rubio, J. Sánchez-Dehesa, F. Meseguer, J. Llinares, and F. Gálvez, Phys. Rev. Lett. 80, 5325 (1998).

${ }^{4}$ E. Yablonovitch, Phys. Rev. Lett. 58, 2059 (1987); S. John, ibid. 58 (23), 2486 (1987)

${ }^{5}$ R. Zengerle, Jour. Mod. Opts. 34, 1589 (1987)

${ }^{6}$ I. Pérez-Arjona, V. J. Sánchez-Morcillo, J. Redondo, V. Espinosa, and K. Staliunas, Phys. Rev. B 75, 014304 (2007).

${ }^{7}$ E. Soliveres, V. Espinosa, I. Pérez-Arjona, V. Sánchez-Morcillo and K. Staliunas, App. Phys. Lett. 94, 164101 (2009).

${ }^{8}$ J. Bucay, E. Roussel, J. Vasseur, P. Deymier, A.-C. HladkyHennion, Y. Pennec, K. Muralidharan, B. Djafari-Rouhani, and B. Dubus, Phys. Rev. B 79, 214305 (2009).

${ }^{9}$ C. Luo, S. G. Johnson, J. Joannopoulos, and J. B. Pendry, Phys Rev. B 68, 045115 (2003).

${ }^{10}$ A. Sukhovich, B. Merheb, K. Muralidharan, J. O. Vasseur, Y. Pennec, P. A. Deymier, and J. H. Page, Phys. Rev. Lett. 102, 154301 (2009).

${ }^{11}$ V. Sánchez-Morcillo, K. Staliunas, V. Espinosa, I. Pérez-Arjona, J. Redondo, and E. Soliveres, Phys. Rev. B 80, 134303 (2009).

${ }^{12}$ K. Staliunas and V. Sánchez-Morcillo, Phys. Rev. A 79, 053807 (2009).

${ }^{13}$ L. Maigyte, T. Gertus, M. Peckus, J. Trull, C. Cojocaru, V. Sirutkaitis, and K. Staliunas, Phys. Rev. A 82, 043819 (2010)

${ }^{14}$ R. Picó, V. J. Sánchez-Morcillo, I. Pérez-Arjona, and K. Staliunas, arXiv:1003.4844(2011).

${ }^{15}$ The commercial package COMSOL Multiphysics 3.5 a was used. 


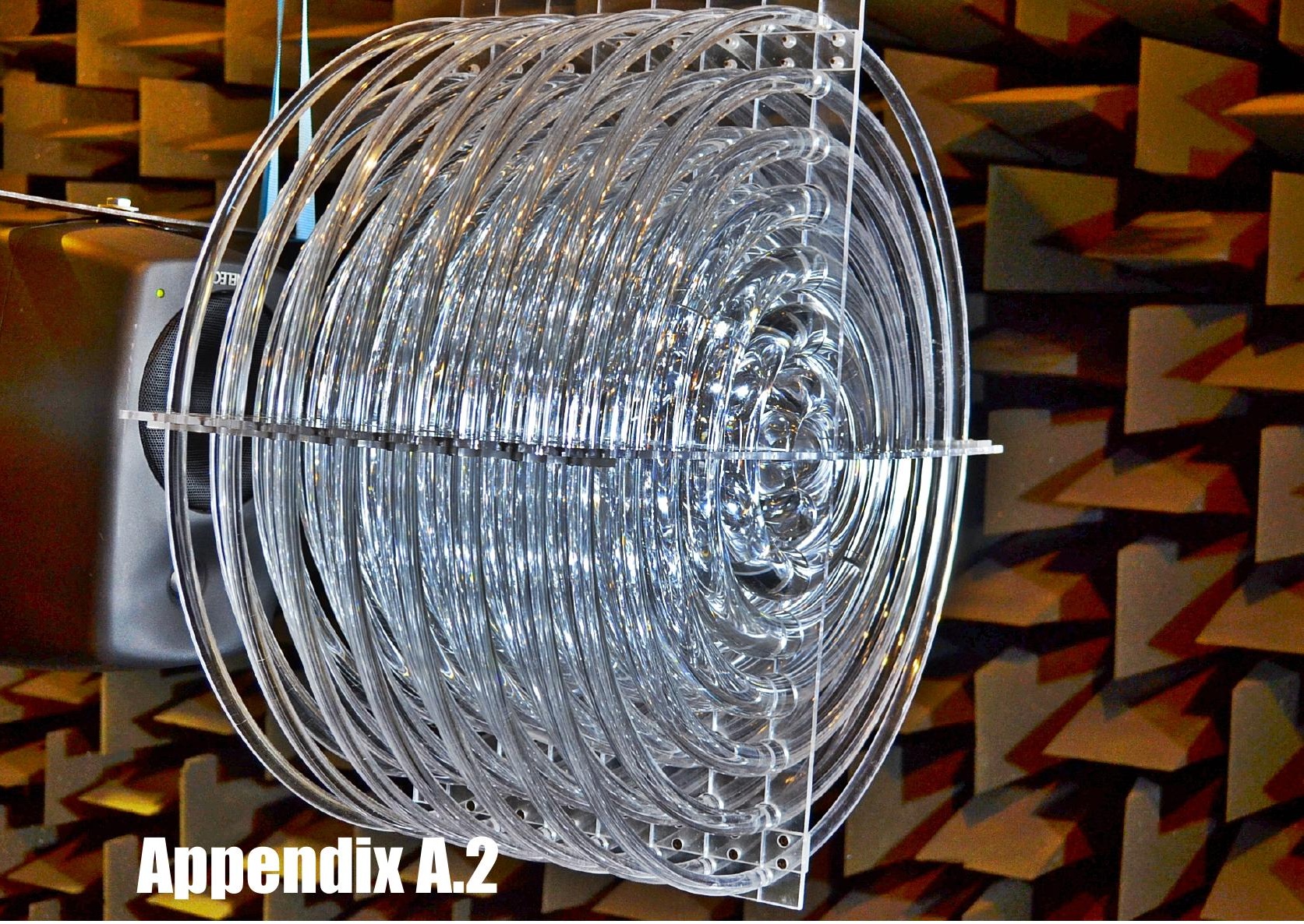

\section{Wave Focusing Using Symmetry Matching in Axisymmetric Acoustic Gradient Index Lenses}

The design and modelling of an axisymmetric gradient index (GRIN) lens made of toroidal scatterers embedded in air is presented here. The system is designed to work in the long wavelength regime where it can be considered as an equivalent medium with effective properties, characterized by the theory of homogenization. The numerical and experimental results presented here demonstrate a remarkable sound amplification. In this regard, axisymmetric structures are good candidates for the development of technological applications. The author of this thesis designed the sample for its fabrication and collaborated in numerical simulations and experimental measurements. 


\title{
Wave focusing using symmetry matching in axisymmetric acoustic gradient index lenses
}

\author{
V. Romero-García, ${ }^{1, \text { a) }}$ A. Cebrecos, ${ }^{2}$ R. Picó, ${ }^{2}$ V.J. Sánchez-Morcillo, ${ }^{2}$ L.M. Garcia-Raffi, ${ }^{3}$ and J.V. \\ Sánchez-Pérez ${ }^{4}$ \\ ${ }^{1)}$ LUNAM Université, Université du Maine, CNRS, LAUM UMR 6613, Av. O. Messiaen, 72085 Le Mans, \\ France \\ ${ }^{2)}$ Instituto de Investigación para la Gestión Integrada de zonas Costeras, Universitat Politècnica de València, \\ Paranimf 1, 46730, Grao de Gandia, València, Spain \\ ${ }^{3)}$ Instituto Universitario de Matemática Pura y Aplicada. Universitat Politècnica de València, Camino de Vera $s / n$, \\ 46022, València, Spain. \\ ${ }^{4)}$ Centro de Tecnologías Físicas: Acústica, Materiales y Astrofísica. Universitat Politècnica de València, \\ Camino de Vera s/n, 46022, València, Spain.
}

The symmetry matching between the source and the lens results of fundamental interest for lensing applications. In this work we have modeled an axisymmetric gradient index (GRIN) lens made of rigid toroidal scatterers embedded in air considering this symmetry matching with radially symmetric sources. The sound amplification obtained in the focal spot of the reported lens ( $8.24 \mathrm{~dB}$ experimentally) shows the efficiency of the axisymmetric lenses with respect to the previous Cartesian acoustic GRIN lenses. The axisymmetric design opens new possibilities in lensing applications in different branches of science and technology.

PACS numbers: 43.20.Fn, 43.20.Gp, 43.20.Mv, 63.20.-e

Photonic $^{1,2}$ and phononic ${ }^{3,4}$ crystals have been revealed in the last years as promising alternatives to control the propagation of electromagnetic and acoustic waves respectively and, based on new physical concepts, with extensive applications in both optics ${ }^{5}$ and acoustics $^{6}$. Depending on the ratio between the wavelength of the incident wave, $\lambda$, and the lattice constant of the crystals, $a$, the basic mechanism describing the action of the crystal on the wave can be best interpreted in terms of refraction ${ }^{7}$ or diffraction ${ }^{9}$. In the long wavelength regime, i.e., $\lambda>>a$, crystals can be considered as homogeneous materials with effective properties ${ }^{10,11}$, therefore one can design refractive ${ }^{7}$ or gradient index (GRIN) ${ }^{12}$ lenses to control waves. In this direction, metamaterial acoustic GRIN lenses have recently been designed by using unit cells based on cross-shape scatterers ${ }^{13}$ and on coiling up space ${ }^{14}$, providing a high transmission efficiency and small size. On the other hand, the case $\lambda \simeq a$ corresponds to diffractive regime, where the crystal is strongly dispersive. Yang et al. ${ }^{15}$ reported the first three dimension (3D) phononic crystal showing the focusing of ultrasonic waves in this regime. Since then several phononic lenses have been designed by using the curvature properties of the isofrequency contours, making use of the all angle negative refraction ${ }^{16}$ and the convex isofrequency contours ${ }^{17}$.

In most of the practical situations the sound wave sources have radial symmetry. Examples can be found in domains as aeroacoustics, microfluidics or medical ultrasound. In this situation the symmetry of the lens becomes relevant and one should consider the full source-

\footnotetext{
a) Instituto de Investigación para la Gestión Integrada de zonas Costeras, Universitat Politècnica de València, Paranimf 1, 46730, Grao de Gandia, València, Spain
}
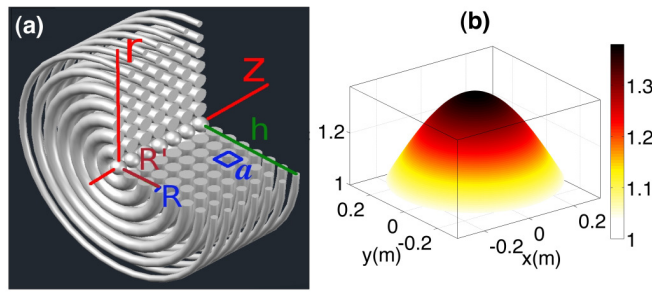

FIG. 1. (Color online) (a) Parameters used in the axisymmetric GRIN lens. (b) Axysimmetric distribution of the refraction index used in the axisymmetric GRIN lens.

lens system in order to improve the efficiency of the joint focusing device. Most of the focusing mechanisms described above have been conceived for cartesian lenses (those presenting translational symmetry, as for example a squared array of cylinders), which do not match with the radial symmetry of the source. A cartesian lens in general match with a semi-infinite rectangular radiating surface, which in the asymptotic limits, corresponds to a plane (radiating an unbounded plane wave) or to a line (radiating a cylindrical beam). The axisymmetric lenses however present a symmetry matching with radial symmetric sources as, for example, the circular radiating piston. The asymptotic limits of this circular radiating piston are the infinite radiating plane (radiating an unbounded plane wave) and the point sources.

Some recent works introduce axisymmetric discrete systems ${ }^{21,22}$ with the aim of focalizing optical or acoustical waves. In the refractive regime, a transformational design of an axial symmetric three-dimensional GRIN 

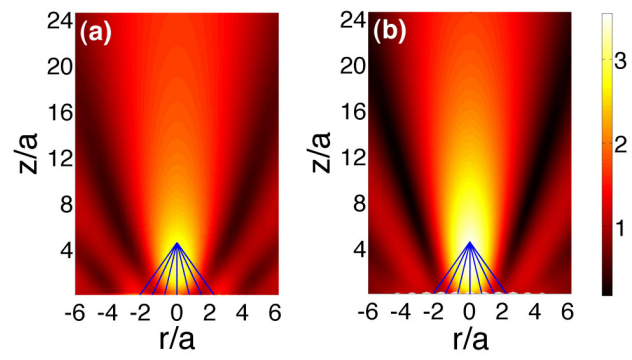

FIG. 2. (Color online) Comparison between the acoustic field (absolute value of pressure, $|p|$ ) behind the equivalent fluid lens (a) and the axisymmetric GRIN lens (b) at $\lambda=4.25 a$. Blue lines represent the ray-tracing trajectories calculated from the derivative of Eq. (4) at the interface of the GRIN lens and then considering Snell's law of refraction.

lens was theoretically proposed in Ref. [21]. On the other hand, in the diffractive regime, in Ref. [22] the authors propose to maximize the focusing properties in a 2D system of cylindrical rigid scatterers embedded in air and obtain, by rotation of the optimized structure, an axial symmetric lens formed by rigid toroidal scatterers. The structure was validated experimentally and the obtained sound amplification in the focus was remarkably high, showing that the rotational symmetry of the system increases its efficiency. However, the structure was designed and optimized for a cartesian system, where the wave equation is different than that of the axisymmetric case. In the axisymmetric situation the equation presents a term proportional to $1 / r$, making the symmetry axis of singular relevance.

In this work we propose the model and the experimental realization of an axisymmetric GRIN lens working with a circular piston source radiating Gaussian beams in the long wavelength regime $(\lambda>4 a)$. In this range of frequencies the axisymmetric lens can be considered as an equivalent fluid. Due to the symmetry matching between the radiated beam and the GRIN lens, a high sound level is found in the focus spot. We have characterized the focusing properties of the complete system, demonstrating values of the sound amplification (gain) higher than those obtained previously with acoustic GRIN lenses. The lens is modeled in the axial plane (horizontal plane) without loss of generality as shown in Fig. 1(a), and it is made of rigid toroidal scatterers embedded in air. Each scatterer is represented by a major radius, $R^{\prime}$, and a minor radius, $R$. In the axial plane the distance between neighbor scatterers is $a$, forming a square array as shown in Fig. 1(a).

In the long wavelength regime, the minor radius of each scatterer can be selected to fix the filling fraction, $f(r)=\pi R(r)^{2} / a^{2}$, at a position $r$ from the center of the lens $^{7}$, and the index of refraction, $n(r)$, can be written in
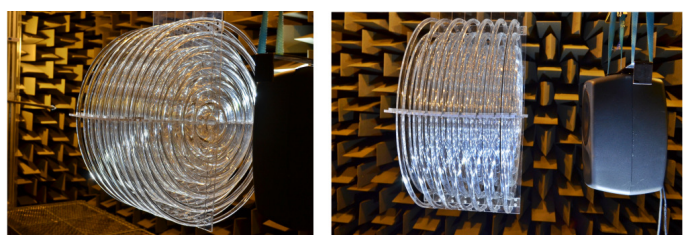

FIG. 3. (Color online) Views of the experimental set-up showing the lens-source system.

terms of $f$ as $^{7,8}$

$$
n(r)=\frac{c_{\text {host }}}{c_{\text {eff }}}=\sqrt{1+f(r)} .
$$

Then, by a gradual change of the filling fraction we can design a refraction index profile in the vertical plane of the lens, perpendicular to the axial $z$-direction. In this work we use the hyperbolic secant profile that has been proved to reduce the aberration of the focal $\operatorname{spot}^{23}$, defined as

$$
n(r)=n_{0} \operatorname{sech}(\alpha r),
$$

where $n_{0}=n(r=0)$ is the refractive index on the $z$-axis $(r=0)$ and $\alpha$ is the gradient coefficient,

$$
\alpha=\frac{1}{h} \cosh ^{-1}\left(\frac{n_{0}}{n_{h}}\right),
$$

with $h$ the half-height of the lens, and $n_{h}$ the refraction index at the lens edges $(r= \pm h)$. Figure 1(b) shows the hyperbolic secant refractive index profile, where we have selected $n_{0}=1$ and $n_{h}=1.33$, being $h=7 a$ the thickness of the lens, so we have used a sample with 7 planes of toroidal scatterers as shown in Fig. 1(a).

The numerical results are obtained by solving the acoustic wave equation using the finite element method (FEM) applied in an axisymmetric domain surrounded by perfectly matched layers, in order to simulate the Sommerfeld radiation conditions. The scatterers are assumed acoustically rigid (infinite impedance), corresponding to Neumann boundary conditions at the interface with the fluid. The incident field was generated by a circular piston of diameter $\mathrm{D}=5 \mathrm{a}$ placed at a distance of $5 \mathrm{a}$ from the source. Figure 2 shows the comparison of the acoustic field behind an axisymmetric slab of thickness $7 a$ made of the equivalent fluid medium with the index profile defined by Eq. (2) (see Fig. 2(a)) and the acoustic field behind the real axisymmetric structure made with toroids shown in Fig. 1(a) at $\lambda=4.25 a$ (see Fig. 2(b)). In both cases, the hyperbolic secant refractive index profile was used to determine the ray-tracing trajectory within GRIN lens as follows ${ }^{12}$ :

$$
y(x)=\frac{1}{h} \sinh ^{-1}\left(u_{0} H_{f}(x)+\frac{\mathrm{d} u_{0}}{\mathrm{~d} x} H_{a}(x)\right),
$$

where $u_{0}=\sinh y_{0} ; H_{a}(x)=\sin (\alpha x) / \alpha$ and $H_{f}(x)=$ $\cos (\alpha x)$. Taking the derivative of Eq. (4) at the interface 

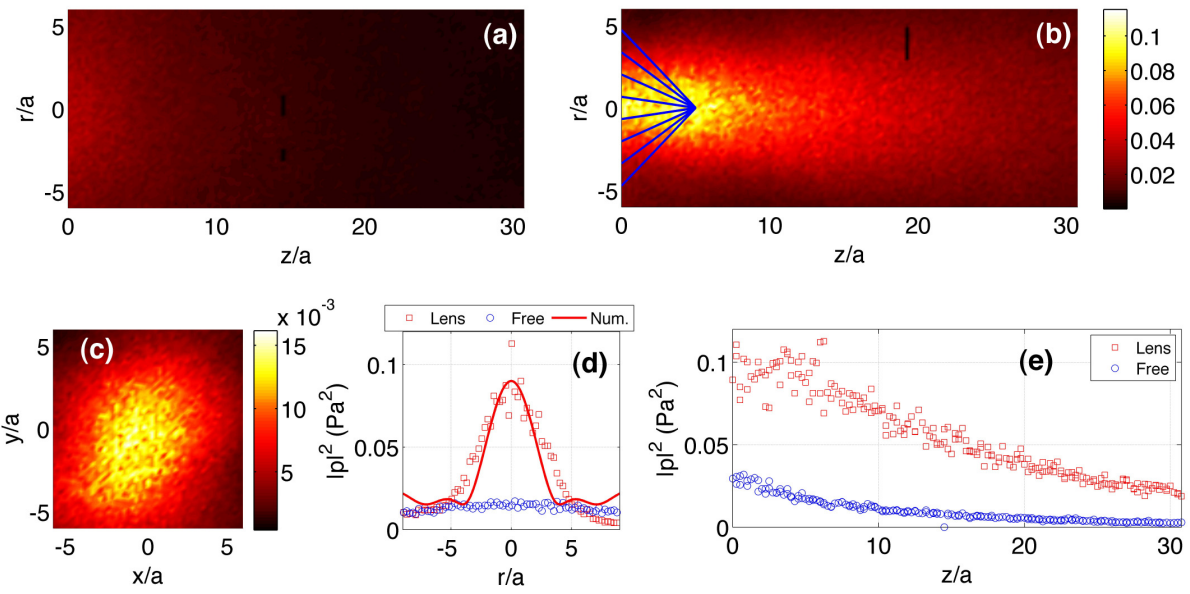

FIG. 4. (Color online) Experimental results of the axisymmetric GRIN lens. (a) and (b) show the experimental intensity axial map $\left(|p|^{2}\right)$ without and with the lens respectively at $\lambda=4.4 a$. Blue lines in (b) show the focal spot position obtained by using the ray-tracing calculations. (c) shows the experimental intensity radial map at the position of the focus. (d) and (e) show the transversal (at $z / a=5$ ) and longitudinal (at $r / a=0$ ) cuts respectively. Blue open circles (red open squares) show the intensity in free field (behind the axisymmetric GRIN lens). As an eye-guide for comparison the red continuous line represents the numerical simulation results obtained using FEM.

of the GRIN lens and considering Snell's law of refraction one can obtain the focus position behind the GRIN lens. Blue continuous lines in Fig. 2 represent the ray-tracing trajectories behind the samples for the case considered in this work, showing the focal point at $z \simeq 5 a$. A good agreement between simulations for the equivalent fluid, Fig. 2(a), and the real structure, Fig. 2(b), is observed.

An experimental set-up was designed to characterize the focusing properties of the axisymmetric GRIN lens in an anechoic chamber and to obtain quantitative data of the acoustic field behind the lens. The dimensions of the echo-free chamber are $8 \times 6 \times 3 \mathrm{~m}^{3}$. The automatized acquisition system 3DReAMS (3D Robotized eAcoustic Measurement System $)^{24}$ was used to scan the acoustic field distribution. Both the source and the lens were hanged and accurately oriented. We notice that the system is based on the axisymmetric properties, so the alignment between the source and the lens is found critical to experimentally obtain a high sound level in the focal spot. Figure 3 shows two views of the experimental set-up. The toroidal scatterers of the lens are made of plexiglass, which acoustic impedance is $\sim 6000$ times bigger than that of the air. Therefore, the toroidal scatterers can be considered acoustically rigid. A loudspeaker is excited with a white noise signal. The diameter of the circular source is $D=5 a$ and it is placed at a distance of $5 a$ from the lens. In this work we present all the results in normalized units with respect to the lattice constant of the sample, so it is worth noting that for our experiments we have used $a=4 \mathrm{~cm}$.

The acoustic axisymmetric GRIN lens has been de- signed to operate as a focusing device in the homogenization regime. The lens presents a broadband behavior in the low frequency range, with modulations in amplitude due to the Fabry-Pérot resonances of the slab. Here we evaluate the intensity maps $\left(|p|^{2}\right)$ at $\lambda=4.4 a$. We measured the case of free propagation (Fig. 4(a)) and the case of the propagation through the GRIN lens in the axial (Fig. 4(b)) and the radial planes (4(c))). As in Fig. 2, the blue continuous lines in Fig. 4(b) represent the ray-tracing trajectory behind the sample showing the focal spot at $z \simeq 5 a$ in good agreement with the predictions shown in Fig. 2.

Figures 4(d) and 4(e) represent the experimental transversal (at $z / a=5$ ) and longitudinal (at $r / a=0$ ) cross-sections respectively. Blue open circles and red open squares show the intensity in free propagation and behind the axisymmetric GRIN lens respectively. One can observe a considerably gain in the symmetry axis with respect to the case of free field propagation. Red continuous line in Fig. 4(d) represents the numerical transversal cut obtained using FEM. Using the data of Fig. 4(d) we can quantitatively characterize the sound amplification (SA) produced in the focal point as well as the Full Width at Half Maximum (FWHM). From Fig. $4(\mathrm{~d})$ the $\mathrm{FWHM}=1.04 \lambda$. Considering as a reference pressure the value of the experimental measurement in free field, $|p|_{\text {free }}^{2}=0.013 \mathrm{~Pa}$, we can evaluate the sound amplification in the focal point as $S A(d B)=$ $10 \log _{10}\left(|p|_{\text {lens }}^{2} /|p|_{\text {free }}^{2}\right)=8.24 \mathrm{~dB}$. The high value of $S A$ for the case of the axisymmetric lens obtained in this work is in contrast with the value of the $S A$ obtained 
using cartesian lenses ${ }^{25,26}$. In the case of the cartesian lenses the focus is extended over the third dimension $(z$ goes from $-\infty$ to $+\infty$ ) while in the case of the axisymmetric lenses the focus forms in a finite volume because in this case one dimension is bounded ( $\theta$ goes from 0 to $2 \pi)$. Then, the symmetry matching in axisymmetric structures is revealed of fundamental relevance to increase the focusing properties of the full source-lens system in practical situations.

In the homogenization limit, it is possible to obtain the acoustic impedance of the lens material as a function of the filling fraction as follows,

$$
Z_{e f f}(r)=\frac{\sqrt{1+f(r)}}{1-f(r)} Z_{h}
$$

where $Z_{h}=\rho_{h} c_{h}$ is the acoustic impedance of the host medium $^{7}$ (in the current work, air, $\rho_{h}=1.29 \mathrm{~kg} / \mathrm{m}^{3}$ and $c_{h}=343 \mathrm{~m} / \mathrm{s}$ ). In our design, the maximum impedance contrast appears in the center of the lens $(f(r=0)=\pi / 4)$ and its value is $Z(0)=6.2 Z_{h}$. The impedance profile is governed by the refraction index profile, therefore presenting a decrease of the impedance along the radial coordinate. This impedance profile assures that the acoustic waves are strongly refracted and weakly reflected, which reinforces the high sound focusing obtained by the axisymmetric GRIN lens presented here.

In this work we have designed an axisymmetric GRIN lens presenting a geometry matching with the source. To do that, we have built a system made of rigid toroidal scatterers embedded in air, by varying the filling fraction in the radial plane in order to produce a hyperbolic secant profile. The ray-tracing of the paraxial approximation, the effective fluid medium approximation, the numerical prediction of FEM and the experimental results, all are in good agreement showing enhanced focusing properties never observed before in this kind of GRIN lenses. Sound amplifications of $8.24 \mathrm{~dB}$ have been observed in the focusing spot by our axisymmetric GRIN lenses. This macroscopic lens, due to its axial symmetric design and the geometry matching with most of the acoustic sources could be the motivation for several applications in science and technology ranging from aeroacoustics to microfluidics or ultrasound therapy.

\section{ACKNOWLEDGMENTS}

The work was supported by Spanish Ministry of Science and Innovation and European Union FEDER through projects FIS2011-29734-C02-01 and -02 and PAID 2012/253. V.R.G. is grateful for the support of post-doctoral contracts of the UPV CEI-01-11.

${ }^{1}$ S. John, Phys. Rev. Lett. 58, 2486 (1987).

${ }^{2}$ E. Yablonovitch, Phys. Rev. Lett. 58, 2059 (1987).

${ }^{3}$ M. Kushwaha, P. Halevi, L. Dobrzynski, and B. Djafari-Rouhani, Phys. Rev. Lett. 71, 2022 (1993).

${ }^{4}$ R. Martínez-Sala, J. Sancho, J. V. Sánchez, V. Gómez, J. Llinares, and F. Meseguer, nature 378, 241 (1995).

${ }^{5}$ J. Joannopoulos, S. Johnson, J. Winn, and R. Meade, Photonic Crystals. Molding the Flow of Light (Princeton University press, Princeton, 2008).

${ }^{6}$ Y. Pennec, J. O. Vasseur, B. Djafari-Rouhani, L. Dobrzy?ski, and P. A. Deymier, Surface Science Reports 65, 229 (2010).

${ }^{7}$ F. Cervera, L. Sanchis, J. V. Sánchez-Pérez, R. Martínez-Sala, C. Rubio, and F. Meseguer, Phys. Rev Lett. 88, 023902 (2002).

${ }^{8}$ A. A. Krokhin, J. Arriaga, and L. N. Gumen, Phys. Rev Lett. 91, 264302 (2003).

${ }^{9}$ J. V. Sánchez-Pérez, D. Caballero, R. Mártinez-Sala, C. Rubio, J. Sánchez-Dehesa, F. Meseguer, J. Llinares, and F. Gálvez, Phys. Rev. Lett. 80, 5325 (1998).

${ }^{10} \mathrm{P}$. Sheng, Introduction to Wave Scattering, Localization, and Mesoscopic Phenomena (Academic Press, New York, 1995).

${ }^{11}$ J. Mei, Z. Liu, W. Wen, and P. Sheng, Phys. Rev. Lett. 96, 024301 (2006).

${ }^{12}$ S.-C. Lin, T. Huang, J.-H. Sun, and T.-T. Wu, Phys. Rev. B 79, 094302 (2009).

${ }^{13}$ L. Zigoneanu, B.-I. Popa, and S. A. Cummer, Phys. Rev. B 84, 024305 (2011).

${ }^{14}$ Y. Li, B. Liang, X. Tao, X.-F. Zhu, X.-Y. Zou, and J.-C. Cheng, Appl. Phys. Lett. 101, 233508 (2012).

${ }^{15}$ S. Yang, J. H. Page, Z. Liu, M. L. Cowan, C. Chan, , and P. Sheng, Phys. Rev. Lett. 93, 024301 (2004).

${ }^{16}$ C. Luo, S. G. Johnson, J. D. Joannopoulos, and J. B. Pendry, Phys. Rev. B 65, 201104(R) (2002).

${ }^{17}$ M. Ke, Z. Liu, C. Qiu, W. Wang, J. Shi, W. Wen, and P. Sheng, Phys. Rev. B 72, 064306 (2005).

${ }^{18}$ M. Samimy, J.-H. Kim, M. Kearney-Fischer, and A. Sinha, J. Fluid. Mech 656, 507 (2010).

${ }^{19}$ Y. Choe, J. W. Kim, K. K. Shung, and E. S. Kim, Appl. Phys. Lett. 99, 233704 (2011).

${ }^{20}$ H. W. Baac, J. G. Ok, A. Maxwell, K.-T. Lee, Y.-C. Chen, A. J. Hart, Z. Xu, E. Yoon, and L. J. Guo, Sci. Rep. 2, 989 (2012).

${ }^{21}$ T. M. Chang, G. Dupont, S. Enoch, and S. Guenneau, New J. Phys. 14, 035011 (2012).

${ }^{22}$ L. Sanchis, A. Yánez, P. L. Galindo, J. Pizarro, and J. M. Pastor, Appl. Phys. Lett. 97, 054103 (2010).

${ }^{23}$ C. Gómez-Reino, M. V. Perez, and C. Bao, Gradient-index Optics: Fundamentals and Applications (Springer, Berlin, 2002).

${ }^{24}$ V. Romero-García, J. Sánchez-Pérez, S. Castiñeira-Ibáñez, and L. Garcia-Raffi, Appl. Phys. Lett. 96, 124102 (2010).

${ }^{25}$ A. Climente, D. Torrent, and J. Sánchez-Dehesa, Appl. Phys. Lett. 97, 104103 (2010).

${ }^{26}$ T. P. Martin, M. Nicholas, G. J. Orris, L.-W. Cai, D. Torrent, and J. Sánchez-Dehesa, Appl. Phys. Lett. 97, 113503 (2010). 
Spatial profile of a harmonic plane wave showing the wave enhancement at the bright plane in a chirped sonic crystal.

\section{Appendix 4.3}

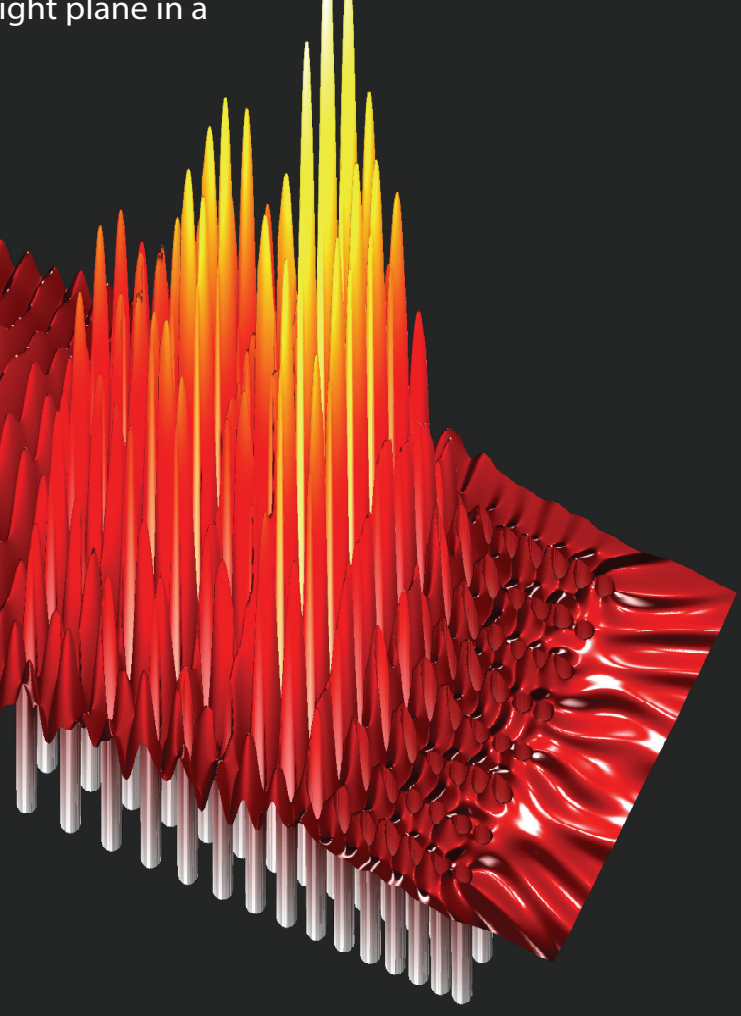

\section{Enhancement of Sound in Chirped Sonic Erystals}

A novel mechanism of sound wave concentration based on the concept of soft reflection is presented in this publication for linear chirped sonic crystals. This effect is related to a progresive slowing down of the sound wave as it propagates along the material and arises at particular planes of the crystal. At these planes, a substantial enhancement of the intensity is obtained for frequencies around the band gap. A coupled mode theory is proposed to predict and interpret the effect and numerical simulations in harmonic regime are performed to estimate the enhancement. Experimental measurements are also carried out and the results are in good agreement with analytical and numerical predictions. The author of this thesis participated in the realization of numerical simulations and experimental measurements. 


\title{
Enhancement of sound in chirped sonic crystals
}

\author{
V. Romero-García, ${ }^{1}$ R. Picó, ${ }^{1}$ A. Cebrecos, ${ }^{1}$ V. J. Sánchez-Morcillo, ${ }^{1}$ and K. Staliunas ${ }^{2}$ \\ 1) Instituto de Investigación para la Gestión Integrada de zonas Costeras, Universitat Politècnica de València, \\ Paranimf 1, 46730, Grao de Gandia, València, Spain \\ ${ }^{2)}$ ICREA, Departament de Física i Enginyeria Nuclear, Universitat Politècnica de Catalunya, Colom 11, \\ E-08222 Terrasa, Barcelona, Spain
}

\begin{abstract}
We propose and experimentally demonstrate a mechanism of sound wave concentration based on soft reflections in chirped sonic crystals. The reported controlled field enhancement occurs at around particular (bright) planes in the crystal, and is related to a progressive slowing down of the sound wave as it propagates along the material. At these bright planes, a substantial concentration of the energy (with a local increase up to 20 times) was obtained for a linear chirp and for frequencies around the first band gap. A simple couple mode theory is proposed, that interprets and estimates the observed effects. Wave concentration energy can be applied to increase the efficiency of detectors and absorbers.
\end{abstract}

PACS numbers: 43.20.Fn, 43.20.Gp, 43.20.Mv, 63.20.-e

Manipulation and control of wave propagation, a problem of fundamental interest, is at root of many applications in different branches of science and technology. One important issue of wave manipulation is the localization and concentration (or local enhancement) of the wave energy. Artificial materials, and among them, artificial crystals are emerging as promising tools for manipulating wave propagation. In the case of sound waves considered here, such artificial periodic materials are called sonic crystals, structurally similar to photonic crystals in the field of optics. They are synthetic materials formed by a periodic distribution of elements or scatterers, whose properties (i.e., elasticity and density) differ from those of the host medium. This results in a periodic modulation of the acoustic properties of the medium at the scale of wavelength. The strong interest in these materials comes from their ability of manipulating the propagation of sound waves, due to their peculiar dispersive properties. A number of exotic and useful effects such as the formation of band-gaps, ${ }^{1,2}$ negative refraction, ${ }^{3}$ birefraction, ${ }^{4}$ self-collimation, ${ }^{5}$ extraordinary transmission, ${ }^{6}$, among others, have been so far demonstrated for sound waves. Utilizing these wave propagation effects, novel devices such as acoustic frequency filters, ${ }^{7}$ spatial (angular) filters, ${ }^{8}$ lenses,${ }^{9}$ or diodes ${ }^{10}$ have been proposed and demonstrated.

We present here a wave propagation effect, consisting in specifically the wave energy concentration due to progressive decrease of the group velocity in chirped sonic crystals, in which the lattice constant, i.e. the distance between scatterers in longitudinal (the wave propagation) direction, gradually changes along the propagation direction. We propose and demonstrate here a substantial increase of the wave intensity in controlled zones inside the crystal. Chirped (sometimes called graded or adiabatic tapered) crystals have been introduced in optics $^{11}$ and acoustics ${ }^{12-14}$ for different purposes, such as opening wide full band gaps in tandem structures ${ }^{12}$ or waveguiding of beams. An intriguing phenomenon shown in chirped crystals is the smooth deflection of a light beam from the straight trajectory as it propagates
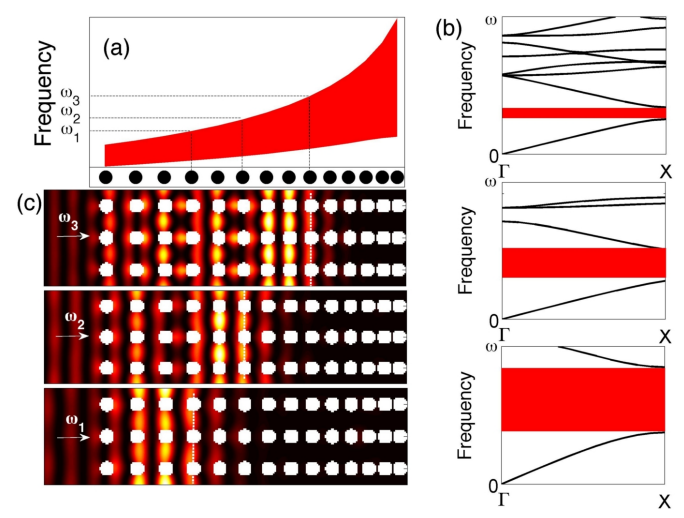

FIG. 1. (Color online) (a) Dependence of the local band gaps on the local lattice constant along the chirped sonic crystal. (b) Band structure (local dispersion curves) evaluated at different depths: at the entrance (top), at the middle (center) and at the exit (bottom) of the sonic crystal. (c) Intensity of the acoustic field calculated using Multiple Scattering technique inside the chirped structure for the frequencies in (a) and (b).

through the crystal, the so-called mirage effect ${ }^{15}$.

Another interesting effect reported recently is the socalled rainbow trapping effect, the dependence of the turning point position on the color of radiation. It has been predicted for one-dimensionally modulated chirped photonic structures ${ }^{16}$ and tapered optical and plasmonic waveguides ${ }^{17,18}$. Rainbow trapping and wave enhancement are two different physical effects (the latter occurs even for monochromatic radiation), although they may occur simultaneously in chirped structures when the incident radiation is broadband. In this letter, in addition to the extraordinary sound wave enhancement effect, which is the main result reported, we also present a sound rainbow trapping effect for acoustic waves as a secondary result. Wave reflection from a band-gap in a chirped structure is peculiar. The dispersion curves $\omega(k)$ at- and close to the band-edges, develop nearly horizontal seg- 
ments, which corresponds to small or zero group velocity of the wave, since $v_{g}=\partial \omega / \partial k$. The occurrence of the controlled sound enhancement requires that the crystal at the entrance plane be within the transparency range for the incoming wave, whose frequency is above the first band-gap, as shown in Fig. 1. Note that here, not the wave frequency but the central (Bragg) frequency and the width of the band-gap is considered variable along the structure. Figures 1(a) and 1(b) show the variation of the band-gaps along the chirped sonic crystal, and the local dispersion curves at different depths, respectively. By local dispersion relation we mean the dispersion of an infinitely extended periodic crystal, for parameters (lattice constant, filling factor) corresponding to a particular depth of the chirped crystal. The wave entering into the crystal is gradually slowing down, as the local band-gaps are approaching the wave frequency in the course of propagation. Finally at a particular depth corresponding to the band-edge, the wave, literally speaking, stops, turns around, and starts propagating back. In other words it experiences a soft reflection. This effect is demonstrated in Fig. 1(c), which shows the wave propagating through the crystal as obtained by numerical simulation using the multiple scattering theory approach ${ }^{19,20}$. The frequencies of the incident waves in the simulations correspond to local band-gaps at different depths. Figure 1(c) evidences that the intensity of the wave increases substantially in the soft reflection area. Most importantly and in opposition to the case of perfectly periodic crystals (constant lattice period) in which only some discrete frequencies can be enhanced by the Fabry-Pérot resonances, chirped crystals can localize the energy for a wide range of frequencies in a controlled way by the gradually change of the lattice constant.

An experimental setup was designed to demonstrate the predicted extraordinary enhancement effect, and to obtain quantitative data of the acoustic field inside the structure. It consists in a two-dimensional sonic crystal with rectangular local symmetry, as illustrated in Fig. 2, made of acoustically rigid aluminum cylinders, of radius $r=2 \mathrm{~cm}$, embedded in air. The spatial period is constant in transverse-to-propagation direction $y, a_{y}=10$ $\mathrm{cm}$, while a longitudinal chirp is introduced in the period along the propagation direction $x$. The adimensional chirp parameter is defined as $\alpha=\left(a_{j}-a_{j+1}\right) / a_{j}$, where $a_{j}$ is the local longitudinal lattice constant at $j$-th layer. For our particular crystal case $a_{0}=10 \mathrm{~cm}$ (initial period), $a_{13}=4.8 \mathrm{~cm}$ (final period), and a gradient $\alpha=0.055$. The sign of the chirp can be either positive or negative, corresponding to lattice constant decreasing or increasing along the propagation direction. In case of identical scatterers, as used in our study, the filling fraction for the positive (negative) chirped structures increases (decreases) in the propagation direction. This has a consequence of broadening of the local bandgap shown in Fig. $1(\mathrm{a})$.

The measurements were performed using the automatized acquisition system 3DReAMS (3D Robotized e-

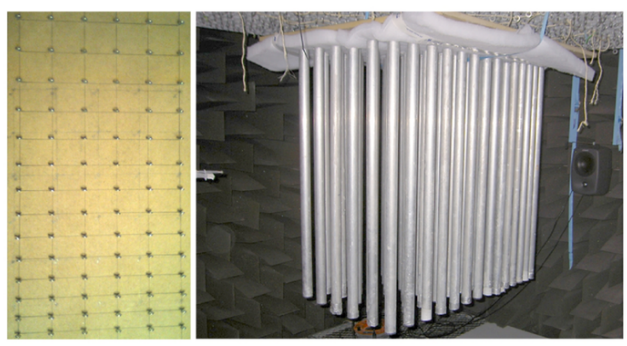

(a)

(b)

FIG. 2. (Color online) Photographs of the experimental setup. (a) the grid of hanging points of the cylinders. (b) the chirped sonic crystal hunging vertically in the anechoic chamber.

Acoustic Measurement System). ${ }^{21}$ Figures 2(a) and 2(b) show the grid of hanging points of the cylinders and a photograph showing the source (a loudspeaker) and the crystal inside the chamber respectively (for propagation directed downwards in Fig. 2(a)). The experimental measurements are in excellent agreement with numerical calculations, as shown in Figs. 3 and 4. We recorded the sound intensity profile along a fixed transverse position by translating the microphone along the $\mathrm{x}$-axis through the void space between the rows of scatterers. In this way, we obtained two-dimensional space-frequency plots as shown in Fig. 3, from numerical (a) and experimental (b) data. White continuous lines mark the positions of the boundaries of the first band gap. Note the concentration of acoustic energy at positions corresponding to just before the upper band edge.

Figure 4(a) shows the axial distributions obtained experimentally (dots) and theoretically (continuous lines) for three particular frequencies. In both cases small-scale fringes are observed, corresponding to the local Bloch mode, as well as a large-scale oscillations or envelope (dashed line) of the Bloch mode, to be discussed below. Figure 4(b) represents the theoretical calculation of the position of the maximum value of concentration of acoustic energy inside the crystal depending on the frequency. In correspondence with the results in Fig. 1, the position of the maximal energy concentration shifts deeper into the bulk of the structure as the frequency is increased (rainbow effect). Note also that, since the incident amplitude was normalized to unity, at the maximum value, the intensity has been recorded up to around 20 times higher than incident. For usual reflection between two different homogeneous media or from a purely band-gap material in the range of the band-gap, only an increase of 4 times of the local intensity is possible (as the interference pattern is formed from forward and fully reflected backward wave). For the case of periodic structures the wave penetrates into the reflecting material evanescently, i.e. with exponential decay ${ }^{21}$ and never shows an increase of intensity. The increase of the intensity field observed 
(a)

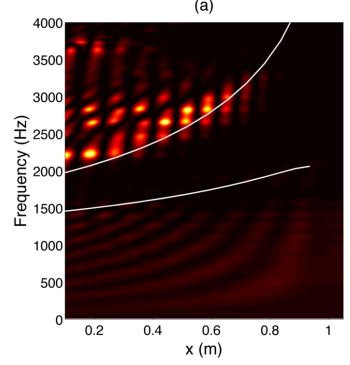

(b)

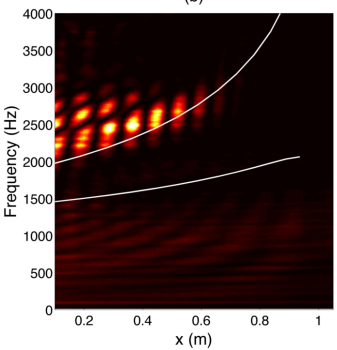

FIG. 3. (Color online) Acoustic intensity inside the crystal, along the $x$-axis, for varying frequencies (a) Multiple Scattering simulation and (b) experiment. White continuous lines show the spatially varying edges of local band-gaps.

in Fig. 4, can be understood from a coupling between the forward and backward waves inside the chirped crystal. In order to interpret the results, we propose a simple coupled mode analytical theory for the propagation of acoustic plane waves inside a one-dimensional chirped crystal (in optics also known as chirped mirror). This dimension reduction is possible because the first band gap in $\Gamma \mathrm{X}$ direction essentially appears due to a resonant coupling between the forward and the backward waves. The contributions of the wave components propagating to transverse directions are negligible. Assuming that the full pressure field consists of forward and backward propagating waves, $P=A(x) e^{\imath k x-\imath \omega t}+B(x) e^{-\imath k x-\imath \omega t}+$ c.c. the following coupled amplitude equations can be systematically obtained from wave equations,

$$
\begin{array}{r}
\frac{d A}{d x}=\imath \frac{\sqrt{s}}{a(x)} B e^{2 \imath \Delta q(x) x}, \\
\frac{d B}{d x}=-\imath \frac{\sqrt{s}}{a(x)} A e^{-2 \imath \Delta q(x) x},
\end{array}
$$

where $s$ is the back-reflection coefficient by one row of scatterers, $a(x)$ the variable longitudinal period and $\Delta q(x)=2 \pi / \lambda-\pi / a(x)$ is the detuning from the Bragg frequency.

From the numerical study of the scattering by only one row of the structure, we estimate that the back reflected intensity is around $40 \%$ of the incident, so $s \simeq 0.4$. The same numerical study reveals that the scattering into field components propagating at transverse direction is only around $5 \%$, which justifies the followed onedimensional approach, neglecting transverse modulations in the vicinity of the first bandgap. We notice that the detuning from the Bragg resonance $\Delta q(x)$ is a function of the longitudinal position $\mathrm{x}$ for chirped crystals. Recall that in our study the chirp is linear, given by $a(x)=a_{0}+\alpha\left(x-x_{0}\right)$.

Equations (1) can be rewritten in canonical form as

$$
\frac{d^{2} A}{d X^{2}}=\imath \epsilon(x) \frac{d A}{d X}+A,
$$

(a)

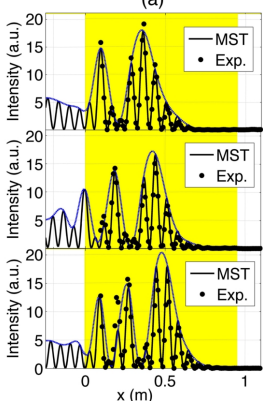

(b)

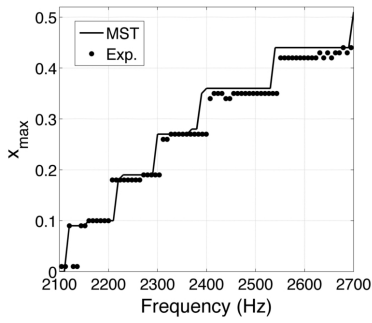

FIG. 4. (Color online) (a) Numerical simulation results (continuous line) and experimental results (dots) for the acoustic intensity at the central section inside the crystal for the three frequencies: $2500 \mathrm{~Hz}, 2600 \mathrm{~Hz}$, and $2700 \mathrm{~Hz}$. The shaded (yellow) rectangle denotes the area covered by the crystal. (b) Position of the maximum value of concentration of energy inside the crystal depending on the frequency of the incident wave.

where the space scaling $d X=d x \sqrt{s} / a(x)$ was chosen to make the normalized coupling coefficient unity, and $\epsilon(x)=2 d(X \Delta q(X) / d X$ is the normalized detuning from the Bragg frequency.

The wave, roughly speaking, reflects from the bandgap, i.e. from the position $X_{0}$ corresponding to the Bragg frequency, with $\epsilon\left(X_{0}\right)=0$. In general (for arbitrary chirp) Eq. (2) cannot be solved analytically. However, in a simple case when the normalized detuning varies linearly around zero $\epsilon(X)=\epsilon_{1}\left(X-X_{0}\right)$, Eq. (2) has an analytical solution in the form

$$
A(X)=c_{1} H_{\imath / \epsilon_{1}}\left(X \sqrt{\imath \epsilon_{1} / 2}\right),
$$

where $H_{n}$ is the Hermite polynomial of imaginary order. The counter-propagating field obeys a similar expression. The integration constant $c_{1}=H_{\imath / \epsilon_{1}}\left(X_{F} \sqrt{\imath \epsilon_{1} / 2}\right)$ is determined by the boundary conditions, by imposing that the amplitude of the forward wave at the front face $X=X_{F}$ equals unity. $\epsilon_{1}=d \epsilon(X) /\left.d X\right|_{X=X_{F}}$ or, in terms of initial variables, $\epsilon_{1}=4 \pi \alpha / s$, which estimated for experimental parameters results $\epsilon_{1}=3$.

In Fig. 5 we present the amplitude of the acoustic intensity of the forward and backward waves for linearly chirped crystals as follows from Eq. (3). The acoustic field is nearly exponential in the bandgap, and oscillatory in front of it. The oscillations, with the period and amplitude increasing as the wave approaches the band-gap, are large-scale oscillations, which originate from the energy exchange between the forward and backward waves. These large-scale oscillations correspond to oscillations of the envelope of the Bloch modes observed in Fig. 4, and are not due to conditions imposed at the entrance of the sonic crystal, e.g. some possible impedance mismatch.

The controlled field enhancement effect is clearly visible again in Fig. 5. From the analytical estimations in Eqs. (1)-(3) and from Fig. 5 it follows that for maximal field enhancement of the wave intensity, the chirp must 

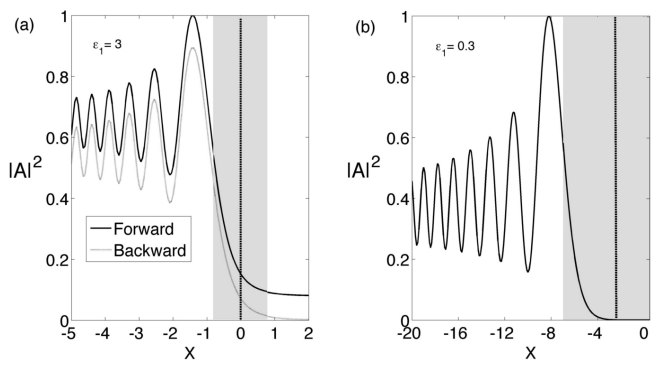

FIG. 5. Solutions of (3), i.e. the intensity of the forward (continuous line) and backward (dashed line) field along the chirped structure. (a) (with $\epsilon=3$ ) corresponds to experimental configuration, (b) (with $\epsilon=0.3$ ) is shown for comparison, to illustrate soft reflections for a substantially smaller chirp. The vertical dashed lines indicate the center of the bandgap, and the shaded areas the bandgap itself.

be as small as possible. For $\epsilon_{1}=0.3$ the maximum field enhancement could be around 6 times (in terms of intensities) if one compares the maximal and minimal values of the plot in Fig. 5(b). In order to realize such enhancement the entrance to the sonic crystal must be placed to correspond to the deepest minimum of the solution (3), in this particular case at around the point $X \simeq 10$. For the parameters of the experiment, $\epsilon_{1}=0.3$, the enhancement of more than two times is predicted in this simplified approach. Also, as Fig. 5(a) shows, a small portion of radiation is transmitted, i.e. leaks through the band gap. Such tunneling, analogous to Landau-Zenner tunneling, is due to slightly too fast (no more adiabatic) chirp.

Concluding, in this Letter we have predicted and experimentally demonstrated a mechanism for sound field enhancement in a chirped crystals, specifically in chirped sonic crystals. The acoustic wave energy can be selectively concentrated at particular depth of the crystal depending on the frequency and on the parameters of the structure. At these bright planes, a substantial increase of the energy was recorded for linear chirp and for frequencies around the first gap along the $\mathrm{X}$ direction of structure. The experimental study was performed in a macroscopic sonic crystal irradiated by acoustic waves in audible regime, where the measurements in the interior of the crystal are possible.

In the field of acoustics the results are independent of the spatial scale of the structure, and in principle the phenomenon could be scaled-down and observed in microor nano-scale phononic (so called hypersonic) crystals ${ }^{22}$. At these scales sound waves are described in terms of phonons, and the ideas presented in this work could find application for heat management in acoustical or acousto-optical devices. Recent works in this direction show indeed that manipulation of phonon dispersion properties can allow thermal transport control ${ }^{23}$. Generally, the effect of wave energy concentration demonstrated in the present work, opens a possibility of increasing the efficiency of detectors and absorbers, both in acoustics and optics, since slow phonons and photons can be absorbed and harvested with a higher probability.

\section{ACKNOWLEDGMENTS}

The work was supported by Spanish Ministry of Science and Innovation and European Union FEDER through projects FIS2011-29734-C02-01 and -02 and GVA/2011/055. V.R.G. is grateful for the support of post-doctoral contracts of the UPV CEI-01-11. K.S. acknowledges the grant of UPV PAID-02-01. We acknowledge the CTFAMA and the Sonic Crystal Technologies Research Group at UPV for the use of the anechoic chamber and the 3DReAMS respectively.

${ }^{1}$ M. Kushwaha, P. Halevi, L. Dobrzynski, and B. Djafari-Rouhani, Phys. Rev. Lett. 71, 2022 (1993).

${ }^{2}$ R. Martínez-Sala, J. Sancho, J. V. Sánchez, V. Gómez, J. Llinares, and F. Meseguer, Nature 378, 241 (1995).

${ }^{3}$ X. Zhang and Z. Liu, Appl. Phys. Lett 85, 341 (2004).

${ }^{4}$ M.-H. Lu, C. Zhang, L. Feng, J. Zhao, Y.-F. Chen, Y.-W. Mao, Y.-Y. Zhu, S.-N. Zhu, and N.-B. Ming, Nature Mat. 6, 744 (2007).

${ }^{5}$ V. Espinosa, V. J. Sánchez-Morcillo, K. Staliunas, I. PérezArjona, and J. Redondo, Phys. Rev. B 76, 140302(R) (2007).

${ }^{6}$ Y. Zhou, M.-H. Lu, L. Feng, X. Ni, Y.-F. Chen, Y.-Y. Zhu, S.-N. Zhu, and N.-B. Ming, Phys. Rev. Lett. 104, 164301 (2010).

${ }^{7}$ A. Khelif, P. A. Deymier, B. Djafari-Rouhani, J. O. Vasseur, and L. Dobrzynski, J. Appl. Phys., Vol. 94, 1308 (2003).

${ }^{8}$ R. Picó, V. Sánchez-Morcillo, I. Pérez-Arjona, and K. Staliunas, Appl. Acoust. 73, 302 (2012).

${ }^{9}$ F. Cervera, L. Sanchis, J. Snchez-Prez, R. Martnez-Sala, C. Rubio, and F. Meseguer, Phys. Rev Lett. 88, 023902 (2002).

${ }^{10}$ X. Li, X. Ni, L. Feng, M. Lu, C. He, and Y. Chen, Phys. Rev. Lett. 106, 084301 (2011).

${ }^{11}$ E. Cassan, C. K.-V. D. Caer, D. Marris-Morini, and L. Vivien, J. Lightwave Tech. 29, 1937 (2011).

${ }^{12}$ M. Kushwaha, B. Djafari-Rouhani, L-Dobrynski, and J. Vasseur, Eur. Phys. J. B 3, 155 (1998).

${ }^{13}$ I. E. Psarobas and M. M. Sigalas, Phys. Rev. B 66, 052302 (2002).

${ }^{14}$ L. Wu and L. Chen, J. Appl. Phys. 110, 114507 (2011).

${ }^{15}$ E. Centeno, D. Cassagne, and J.-P. Albert, Phys. Rev. B 73, 235119 (2006).

${ }^{16}$ Y. Shen, J. Fu, and G. Yu, Phys. Lett. A 375, 3801 (2011).

${ }^{17}$ M. Stockman, Phys. Rev. Lett. 93, 137404 (2004).

${ }^{18}$ V. N. Smolyaninova, I. I. Smolyaninov, A. V. Kildishev, and V. M. Shalaev, Appl. Phys. Lett. 96, 211121 (2010).

${ }^{19} \mathrm{P}$. Martin, Multiple Scattering. Interaction of Time-Harmonic Waves with $N$ Obstcles (Cambirdge University Press, UK, 2006). ${ }^{20}$ Y. Y. Chen and Z. Ye, Phys. Rev. E 64, 036616 (2001).

${ }^{21}$ V. Romero-García, J. Sánchez-Pérez, and L. Garcia-Raffi, J. Appl. Phys. 108, 044907 (2010).

${ }^{22}$ T. Gorishnyy, C. K. Ullal, M. Maldovan, G. Fytas, and E. L. Thomas, Phys. Rev. Lett. 94, 115501 (2005).

${ }^{23}$ P. Hopkins, C. Reinke, M. Su, R. O. III, E. Shaner, Z. Leseman, J. Serrano, L. Phinney, and I. El-Kady, Nano Lett. 11, 107 (2011). 
Snapshot of the spatial profile of a gaussian pulse showing the wave enhancement inside a chirped sonic crystal at a particular time.

\section{ApDendix A.4}

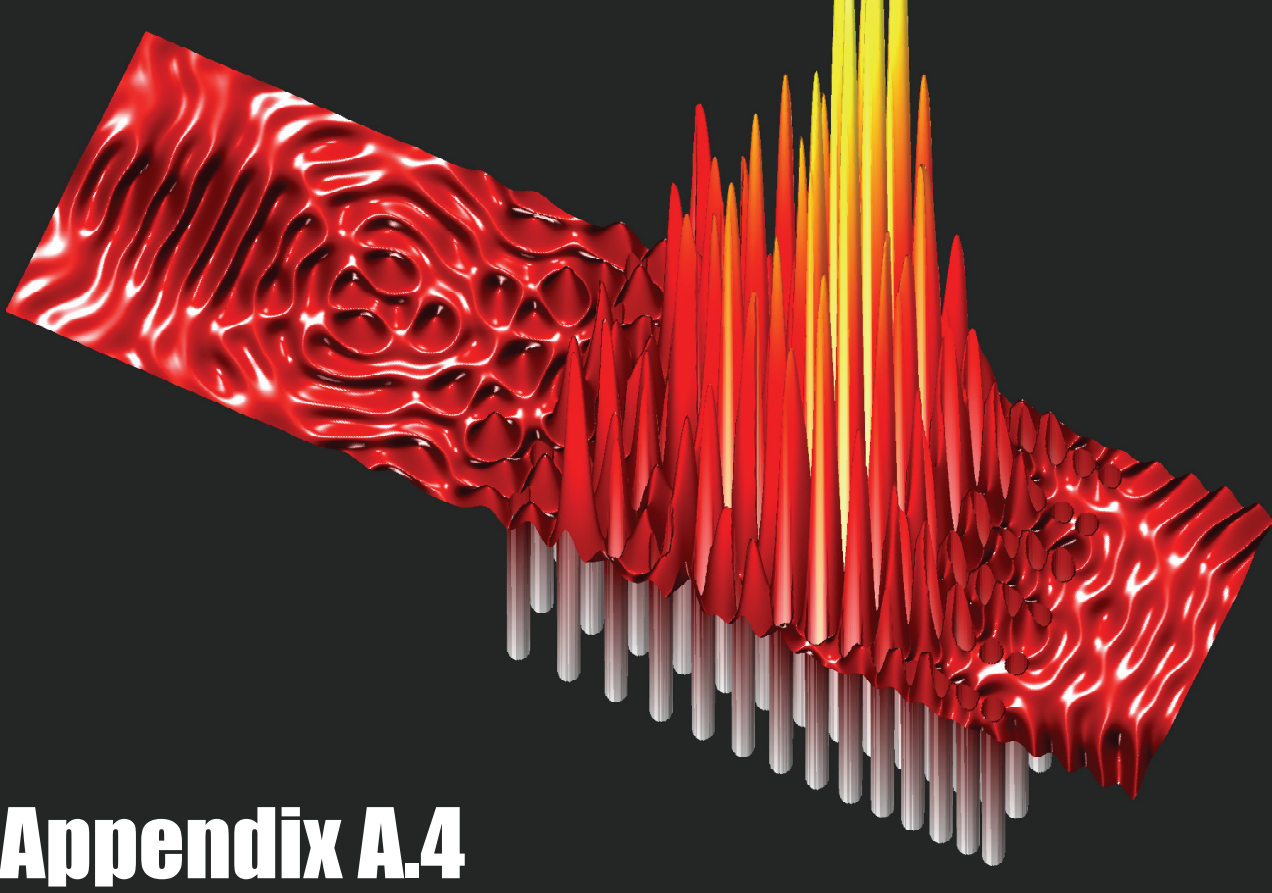

\section{Enhancement of Sound by Soft Reflections in Exponentially Chirped Grystals}

The study of soft reflection in chirped sonic crystals, presented previously, is extended in this work. The analytical predictions extracted from the coupled mode theory are employed here to obtain solutions for a chirped sonic crystal with an exponential variation of the lattice constant, demonstrating that the sound enhancement depends on the variation of the spatial profile. The numerical analysis is expanded to investigate the behavior of the system in time-domain, showing the slowing down of the wave along the propagation direction. The author of this thesis participated in numerical simulations, experimental measurements and writing of the time spreading section. 


\title{
Enhancement of sound by soft reflections in exponentially chirped crystals
}

\author{
A. Cebrecos, ${ }^{1, a}$ R. Picó, ${ }^{1}$ V. J. Sánchez-Morcillo, ${ }^{1}$ K. Staliunas, ${ }^{2}$ \\ V. Romero-García, ${ }^{3}$ and L. M. Garcia-Raffi ${ }^{4}$ \\ ${ }^{1}$ Instituto de Investigación para la Gestión Integrada de zonas Costeras, Universitat \\ Politècnica de València, Paranimf 1, 46730, Grao de Gandia, València, Spain \\ ${ }^{2}$ ICREA, Departament de Física i Enginyeria Nuclear, Universitat Politècnica de Catalunya, \\ Colom 11, E-08222 Terrasa, Barcelona, Spain \\ ${ }^{3}$ LUNAM Université, Université du Maine, CNRS, LAUM UMR 6613, Av. O. Messiaen, \\ 72085 Le Mans, France \\ ${ }^{4}$ Instituto Universitario de Matemática Pura y Aplicada, Universidad Politécnica de Valencia, \\ Camino de Vera s/n, 46022, Valencia, Spain
}

(Received 7 October 2014; accepted 12 November 2014; published online 20 November 2014)

\begin{abstract}
The enhancement of sound inside a two dimensional exponentially chirped crystal during the soft reflections of waves is experimentally and theoretically explored in this work. The control of this enhancement is achieved by a gradual variation of the dispersion in the system by means of a chirp of the lattice constant. The sound enhancement is produced at some planes of the crystal in which the wave is softly reflected due to a progressive slowing down of the sound wave. We find that the character of the sound enhancement depends on the function of the variation of dispersion, i.e., on the function of the chirp. A simple coupled mode theory is proposed to find the analytical solutions of the sound wave enhancement in the exponentially chirped crystal. Harmonic and time domain numerical simulations are performed to interpret the concept of the soft reflections, and to check the analytically calculated field distributions both in good agreement with experiments. Specially we obtain stronger sound enhancement than in linearly chirped crystals. This sound enhancement could motivate applications in energy harvesting, e.g., to increase the efficiency of detectors and absorbers. @ 2014 Author(s). All article content, except where otherwise noted, is licensed under a Creative Commons Attribution 3.0 Unported License. [http://dx.doi.org/10.1063/1.4902508]
\end{abstract}

\section{INTRODUCTION}

During the last decades, many efforts have been done to use periodic structures for the control of wave propagation. Such periodic structures, having a period of the order of the wavelength, are the so-called photonic crystals ${ }^{1}$ for electromagnetic waves and the phononic crystals ${ }^{2}$ for the elastic/acoustic waves. A phononic crystal consists of a periodic distribution of scatterers, whose bulk properties (i.e., elasticity and density) differ from those of the host medium. Based on their dispersion properties, many interesting effects can be observed such as the formation of band-gaps, ${ }^{3,4}$ negative refraction, ${ }^{5}$ birefraction, ${ }^{6}$ self-collimation, ${ }^{7,8}$ extraordinary transmission, ${ }^{9}$ giving rise to novel devices and effects such as spatial ${ }^{10,11}$ and frequency ${ }^{12}$ filters, far field ${ }^{13}$ and near field ${ }^{14}$ focusing, or sound diodes. ${ }^{15}$ Of special interest is the possibility to enhance both spatially and temporally the wave at particular locations inside the crystal, with potential applications as, for example, energy harvesting or enhanced absorption.

Different mechanisms can be used to enhance the waves inside a crystal. At low frequencies (with $\lambda \gg a$, being $\lambda$ the wavelength of the incident frequency and $a$ the distance between the scatterers, or lattice constant), Fabry-Pérot resonances can enhance the wave inside the structure due

\footnotetext{
${ }^{\text {a} E l e c t r o n i c ~ m a i l: ~ a l c e b r u i @ e p s g . u p v . e s ~}$
} 
to the finite thickness of the crystal, $L$, at frequencies related with this thickness, $f=n c / 2 L$ being $n$ an integer number. At higher frequencies, in the highly dispersive regime of the periodic structure in which $\lambda \simeq a$, enhanced localized modes can be excited by the presence of point defects. The periodicity can be locally broken by creating the point defects producing a localized enhanced mode around the defect at a frequency inside the band gap. ${ }^{16,17}$ In both cases, the low frequency and the dispersion regime, the wave can be enhanced for a set of narrow bands (modes), but no broadband enhancement is possible. On the other hand, random systems, in the regime where the wavelength of the incident wave is comparable with the size of the scatterers, present a transition from its diffusion regime to a localized regime, where enhanced localized modes appear in the structure for a broader frequency regime. ${ }^{18,19}$ In this case the strong deviation from Rayleigh statistics is interpreted as a signature of Anderson localization.

Recently, some of us presented an acoustic wave propagation effect, consisting of the wave enhancement due to the progressive decrease of the group velocity along the propagation direction. ${ }^{20}$ Such a progressive slowing-down of the waves was predicted and demonstrated in a twodimensional chirped (also known as graded or adiabatic tapered) sonic crystal, a structure made of rigid scatterers embedded in air in which the lattice constant along the wave propagation direction gradually changes with a profile, $a=a(x)$, depending on the position, $x$. Sound enhancement and slowing down are two related phenomena that occur as the wave approaches to the bandgap; at this position, the waves reaches a zero group velocity and starts propagating backwards, in a proccess that we call a soft reflection. For particular chirp profiles, the wave can be substantially enhanced around the turning plane, whose position inside the crystal depends on the wave frequency. Chirped structures have been also used in optics ${ }^{21,22}$ and acoustics ${ }^{23-25}$ for different purposes such as rainbow trapping, ${ }^{26-28}$ mirage formation, ${ }^{29}$ the opening of wide full band gaps, ${ }^{23}$ or also to control the spatial dispersion and focalizing beams in reflection. ${ }^{22}$

From a practical point of view, it is desirable to obtain the highest enhancement or wave concentration (highest intensity in a shorter distance). The previous study considered a chirped structure with a adiabatic and linear change of the period, ${ }^{20}$ where a moderate enhancement was demonstrated. In this work we explore the properties of a sonic crystal with exponential one. This profile of chirp is of interest because it possess simple analytical solutions, a unique feature that shares with a linear chirp. This allows easier estimations of wave propagation properties depending on system parameters. Most importantly, the exponential chirp is shown to produce a stronger wave enhancement in comparison to the linear profile in Ref. 20. Therefore in this work we report new analytical, numerical and experimental results about a sonic crystal with exponential chirp, and compare the predictions from different methods, showing good agreement between them. Special attention is paid to the interpretation in time domain of the soft reflections produced in the crystal, which are related to the spatial enhancement of the wave in the spatial domain. Simulations are performed in harmonic and time-domain by Finite Element Method (FEM), showing very good agreement with both analytical and experimental results measured in an echo-free chamber.

\section{DISPERSION IN CHIRPED STRUCTURES AND SOFT REFLECTIONS}

Dispersion curves $\omega(k)$ in periodic structures predict very small group velocity $\left(v_{g}=\partial \omega / \partial k\right)$ of the propagating waves close to the band edges, and correspondingly a large dispersion of the incident wave packet. In chirped structures in which the lattice constant gradually varies according to a predetermined profile, the dispersion relation also evolves gradually inside the crystal following the variation of the profile along the structure. The main assumption in these structures is that the variation of the profile is so slow that each point in the chirped crystal can be characterized by a local dispersion relation, which is the dispersion of an infinitely extended periodic crystal with values of the parameters (lattice constant and filling fraction) at the evaluation point. As an example we show in Fig. 1(a) the evolution of the band gap in a chirped crystal with an exponential profile (inset of Fig. 1(a) shows the spatial distribution of the rigid scatterers inside the chirped crystal. See Section IV for more details about the definition of the profile $a=a(x)$ ). In each point inside the chirped crystal we have considered a rectangular array with the local lattice constant and the local filling fraction. Then, using the plane wave expansion, ${ }^{17}$ we have calculated the band structure 


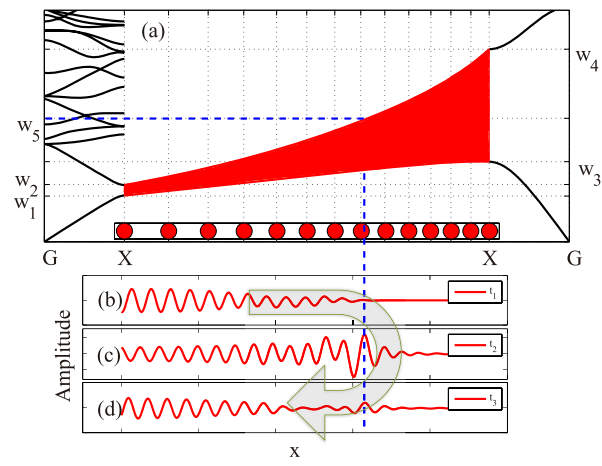

FIG. 1. (a) Local band gaps in exponential chirped structures. Left (right) panel shows the band structures at the entrance (exit) of the system calculated using plane wave expansion. Middle panel shows the evolution of the bandgap within the discrete structure. Inset shows the position of the scatterers inside the system obtained from the exponential chirped shown in Sec. IV. (b), (c) and (d) respectively show the spatial profile of a pulse evaluated at an instant $t_{1}$ before the pulse reaches the turning plane, at the instant $t_{2}$ when the pulse reaches the turning plane and at the instant $t_{3}$ after the pulse reaches the turning plane.

in each point. The number of the plane waves considered for the calculation is enough to ensure the convergence of the plane wave expansion, in our case 1089 plane waves. In the left part, we represent the dispersion relation at the entrance of the crystal, showing the band gap in the range $\left[\omega_{1}=1197, \omega_{2}=1468\right] \mathrm{Hz}$. In the central part of Fig. 1(a), we represent the evolution of the width of the first band gap in the $\Gamma X$ direction along the chirped crystal. As the filling fraction gradually increases along the chirped crystal, the width of the band gap also gradually increases, as shown by the red area in Fig. 1 (a) up to the exit of the crystal, where the width of the band gap $\left[\omega_{3}=2073\right.$, $\left.\omega_{4}=4988\right] \mathrm{Hz}$ corresponds to the periodicity and filling fraction at the exit of the crystal, as shown in the right part of Fig. 1 (a).

In order to have propagation of waves inside the crystal, the frequency of the incident wave must belong to some of the propagating bands of the dispersion relation at the entrance of the crystal. In a general way, we consider now an incident gaussian packet centred at $\omega_{5}$ which, as shown in Fig. 1(a), is in the propagating region at the entrance of the crystal. However, as $\omega_{5}<\omega_{4}$, the wave will arrive to a band gap inside the crystal corresponding to a lattice constant and filling fraction that covers $\omega_{5}$. The wave entering into the crystal is gradually slowing down because, in the course of propagation, it approaches to the edges of local local band gap inside the crystal. At a particular depth corresponding to the band-edge, where the group velocity is zero (in absence of losses), the forward propagating wave stops, turns around, and starts propagating backwards, suffering, what we call here, a "soft" reflection. This reflection effect is observed in the temporal domain in Figs. 1(b)-(d). We show the wave packet at three different instants inside the crystal: (b) before (c) at the instant and (d) after the wave packet reaches the reflecting plane. Figure 1(c) shows how the wave enhancement in the soft reflection area.

\section{COUPLED MODE THEORY: ANALYTICAL RESULTS}

We propose an analytical description of the problem of the enhancement in an exponential chirp profile, based in the so called coupled mode theory. The approach is valid under some approximations. We first notice that at frequencies close to the first band gap, an incident plane wave basically propagates along the direction of incidence (the energy flow along other transversal directions is negligible), and a one-dimensional plane wave description is justified, as shown in Ref. 20. Under such conditions, the medium is roughly equivalent to a multilayered structure, with each plane of scatterers behaving as a layer of a different material. In coupled mode theory, the bandgaps appear as a result of the resonant coupling between the forward and the backward waves. 
Coupled mode theory is applicable for media with periodic modulation of the parameters, but also for a smooth chirp profile (when the period changes adiabatically) and for frequencies close to the band-gap. It is based on the following assumptions: $(i)$ the full pressure field consists of forward and backward propagating waves, $P=A(x) e^{\imath k x-\imath \omega t}+B(x) e^{-\imath k x-\imath \omega t}$, with amplitudes slowly evolving in space, and (ii) the crystal is accounted as a periodic variation of the sound velocity $c$ with the period of the crystal, $c=c_{0}+\Delta c \cos (Q x)$ where $Q=2 \pi / a$. Substituting in the linear wave equation and scaling the space as $X=x / a$, the following coupled amplitude equations system is readily obtained

$$
\begin{aligned}
& \frac{d A}{d X}=i B e^{2 \imath \Delta Q X}, \\
& \frac{d B}{d X}=i A e^{-2 \imath \Delta Q X},
\end{aligned}
$$

where $\Delta Q=a\left(k-k_{B}\right)$ is the detuning from normalized Bragg resonance, with $k_{B}=\pi / a(X)$. After some manipulation, a single second order equation for the slow amplitudes can be obtained, as

$$
\frac{d^{2} A}{d X^{2}}=i m(X) \frac{d A}{d X}+A,
$$

where $m(X)=d(\Delta q X) / d X$ is a slowly varying function of distance, related to the normalized chirp profile $a(X)$ by a simple transformation, which vanishes at Bragg resonance $\Delta q=0$. For several particular cases the above equation has analytical solutions. One case, considered in Ref. 20, corresponds to a linear chirp profile $m(X)=\alpha\left(X-X_{0}\right)$. In this case the field distribution in space is described by a Hermite function with complex index, and presents sound enhancement at planes close to Bragg resonance. We consider here the case of an exponential chirp, in the form $m(X)=e^{b X}-1$. In this case an analytical solution also exists, given by

$$
A(X)=e^{-b X} L_{n}^{s}\left(\frac{i e^{b X}}{b}\right),
$$

where $L_{n}^{s}$ is a generalized Laguerre polynomial, ${ }^{30}$ with the indexes defined as $n=(-1)^{5 / 6} / b$ and $s=\sqrt{3} / b$

In Fig. 2 the profile of the squared amplitude of the wave (normalized intensity) is shown along the chirped structure. We consider here wave incoming form the left side. Continuous blue line represents the results from Eq. (4) for a chirp parameter $b=-0.05$, and green open dots represents

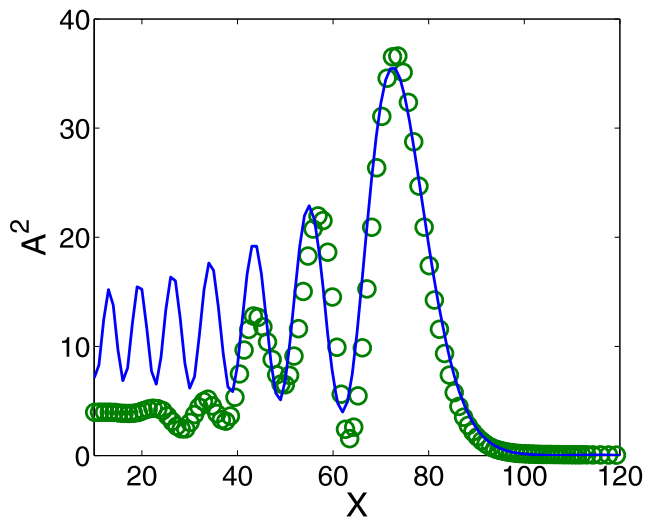

FIG. 2. Continuous line represents the amplitude profile in the exponentially chirped crystal, as given by the analytical solution of Eq. (4) calculated using $b=-0.05$. Green dots represents the envelope for the of the experimental case as shown also in Fig. 4(d). 
the envelope of the experimental data for the case of $2700 \mathrm{~Hz}$. Recall that we represent here the wave envelope, so additional field oscillations with the period of the structure are also present (not shown). The effect of the exponential variation of the lattice constant is evident. A significant enhancement of the wave amplitude appears at the end of the path of the wave (bright plane), just before the turning plane (local band gap). We notice that due to the range of validity of the model, is in the range of frequencies near the band gap in which the theory agrees well with the experiments, as expected. The region in which these oscillations become important is much smaller than in the case of linear chirp (see Fig. 5 (b) in Ref. 20) and the amplitude is more localized. This is one of the main advantages of the chirp profile with respect to the linear one.

\section{EXPERIMENTAL SETUP}

Experimental measurements were carried out with a two-dimensional sonic crystal with rectangular local symmetry, as illustrated in Fig. 3(a). The crystal is made of acoustically rigid aluminum cylinders, with radius $r=2 \mathrm{~cm}$, and height $h=1 \mathrm{~m}$, embedded in air. The spatial period is constant in transverse-to-propagation direction $y, a_{y}=10 \mathrm{~cm}$, whereas an exponential chirp profile is introduced for the period along the propagation direction $x: a_{n}=a_{0} e^{-\alpha x_{n-1}}$, where $a_{0}$ is the lattice constant at the entrance of the chirped structure, $\alpha$ is the exponential chirp parameter and $x_{j}$ the local position in direction $x$ of the $j$-th layer. A chirped crystal formed by 14 rows and 6 columns is considered in this work, with $a_{0}=12.5 \mathrm{~cm}, a_{13}=4.88 \mathrm{~cm}$, and $\alpha=0.01 \mathrm{~m}^{-1}$.

We performed experiments in an echo-free chamber sized $8 \times 6 \times 3 \mathrm{~m}^{3}$ with an automatized acquisition system, 3DReAMS (3D Robotized e-Acoustic Measurement System). ${ }^{16,17}$ This system enables the measurement of pressure fields along complex trajectories as well as inside the crystals. Fig. 3(a) shows the grid of hooks used for hanging cylinders to design the chirped structure which is covered in the setup with an absorbent material to avoid additional reflections in our measurements. Fig. 3(b) shows the negative chirped crystal (decreasing lattice constant) made of Al cylinders inside the echo-free chamber (for propagation directed upwards in Fig. 3(a)) used in this work.

\section{SPATIAL ENHANCEMENT OF THE ACOUSTIC FIELD}

Sound pressure amplitude, $|p|$, was recorded along a line path by moving the microphone along the $x$-axis in the space between two rows of scatterers. The recorded amplitude has been normalized with respect to the amplitude of the incident wave, $\left|p_{0}\right|$. As a result, a two-dimensional
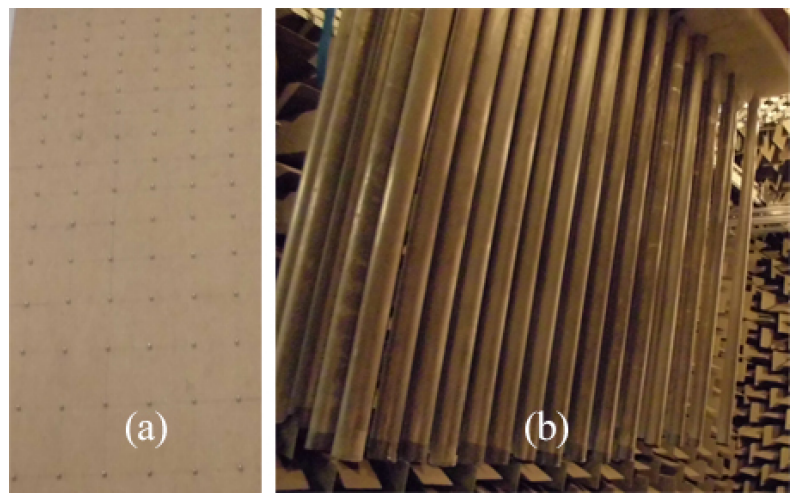

FIG. 3. Photographs of the experimental setup. (a) The grid (14 rows by 6 columns) of hanging points of the cylinders. (b) The chirped sonic crystal made of aluminium cylinders of $1 \mathrm{~m}$ length and $2 \mathrm{~cm}$ radius, hanging vertically in the anechoic chamber. 

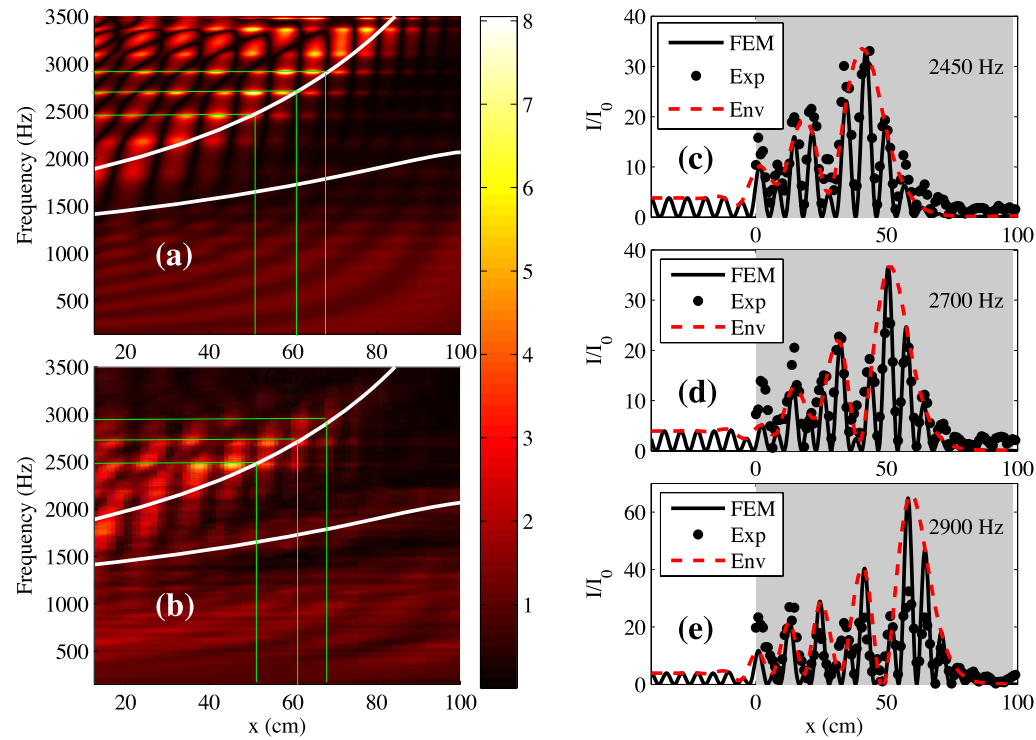

FIG. 4. (a) Numerical simulations and (b) experimental results of the normalized acoustic field with respect to the incident waves, i.e., $|p| /\left|p_{0}\right|$, inside the chirped sonic crystal. White continuous lines define the limits of the upper and lower edges of the first band gap as the periodicity changes inside the structure. Green lines illustrate the frequencies shown in (c), (d), (e) and the corresponding positions of the turning planes inside the crystal. (c), (d) and (e) show the normalized acoustic intensity, $I / I_{0}=|p|^{2} /\left|p_{0}\right|^{2}$ for a longitudinal cut inside the crystal at three frequencies $2450 \mathrm{~Hz}, 2700 \mathrm{~Hz}$ and $2900 \mathrm{~Hz}$ respectively (shown also in (a) and (b)). Grey shaded rectangle denotes the area covered by the exponential chirped crystal.

frequency-space map representing the normalized acoustic field, $|p| /\left|p_{0}\right|$, inside the structure is obtained. The map shown in Fig. 4(a) is numerically calculated using FEM in harmonic analysis and Fig. 4(b) shows the normalized map directly obtained from experimental measurements. As an eye guide, we plot also in Figs. 4(a) and 4(b) the evolution of the band gap along the exponential chirped crystal (white continuous lines), obtained using plane wave expansion as described in Section II. It is worth noting here the wave enhancement at positions just before the upper band edge. We notice here that the turning planes at these frequencies appear at the positions $x=50 \mathrm{~cm}, 60 \mathrm{~cm}$ and $64.5 \mathrm{~cm}$, respectively, as numerically and experimentally shown in Figs. 4(a)-4(b). As expected, the upper edge of the local band gaps corresponds to the turning planes at different frequencies in different locations inside the crystal.

In order to analyze the sound enhancement and its properties, we analyze the cases of three frequencies, 2450, 2700 and $2900 \mathrm{~Hz}$. Figures 4(c)-4(e) show the axial distributions of the normalized intensity, $|I| /\left|I_{0}\right|=|p|^{2} /\left|p_{0}\right|^{2}$, obtained experimentally (dots) and theoretically (continuous lines) for these three frequencies respectively. In both cases, small-scale fringes are observed, corresponding to the local Bloch mode, as well as a large-scale oscillations or envelope (dashed line) of the Bloch mode, as it is previously analyzed in Section III. On the other hand, at the same frequencies, the maximal sound enhancement are placed at $x=43.18 \mathrm{~cm}, 50.48 \mathrm{~cm}$ and $57.68 \mathrm{~cm}$ respectively as it can be seen in Figs. 4(c)-4(d). Therefore, in correspondence with the results shown in Fig. 2 from our coupled mode theory, the position of the bright plane (corresponding to the sound enhancement) for every case is shifted back with respect to the turning plane. Moreover, the position of the maximal sound enhancement shifts deeper into the bulk of the structure as the frequency is increased, which is a clear sign of the acoustic rainbow effect produced by this kind of structures.

We pay attention now to the maximum value of the wave enhancement. Since the plots are normalized with respect to the incident wave, we can see that at the maximum value of wave enhancement, the intensity is around 60 times higher than that of the incident one. This result 
improves the previously obtained for a linear chirped crystal, ${ }^{20}$ which produces an enhancement of 20. We notice here that for usual reflection between two different homogeneous media or from a purely band-gap material in the range of the band-gap, only an increase of 4 times of the local intensity is possible.

The envelopes shown in Figs. 4(c)-(e) with red dashed lines, connect the experimental and numerical results with those obtained with our model based on coupled mode theory. As we previously described in Fig. 2 this red dashed lines correspond to the envelopes of the Bloch modes and they are not due to conditions imposed at the entrance of the sonic crystal as for example some possible impedance mismatch.

\section{TIME SPREADING}

In this part we analyze the effects of chirped lattice constant on the wave propagation evaluated in time domain, i.e. the effects on the character of the time spreading of the pulse reflected from the chirped structure. For that purpose, FEM simulations in time domain were carried out for the three frequencies previously mentioned, 2450,2700 and $2900 \mathrm{~Hz}$. For the simulations we use a gaussian pulse centered at these frequencies with fixed bandwidth $\Delta \omega=100 \mathrm{~Hz}$. During the simulations we consider here that the gaussian beam propagates from left to right.

Figure 5 shows the time-space scenario for $f=2700 \mathrm{~Hz}$, where the sound pressure amplitude, $p$, is shown. Figure 5(a) shows the pulse propagating in air and reflecting from a rigid wall placed at the position corresponding to the turning plane of the chirped structure for this specific frequency. As expected, the relation between $x$ and $t$ is a straight line and its slope is given by the sound velocity in air. The amplitude is modulated in $x$ due to the interference between the incident and reflected wave.

Figure 5(b) shows the pulse propagating in air from $x=[0,1] \mathrm{m}$, and along the chirped structure (placed in the range $x=[1,2] \mathrm{m}$ ). The effect of soft reflection is clearly observed here. The incident wave is reflected first at the turning plane corresponding for the central frequency, around $x=1.6 \mathrm{~m}$, producing the bright plane just a bit before due to the reduction of the group velocity (as shown in previous Sections). After this first reflection, the pulse travels back out of the structure. In contrast to the rigid wall case, the wave here is reflected back and forth between the entrance and the turning planes of the chirped structure, giving rise to multiple contributions propagating back.

Time profiles of the signals are shown in Figs. 5(c) and 5(d) evaluated at the positions marked by the green vertical lines in Figs. 5 (a) and (b). Whereas the recorded signal for the case of a rigid wall is the superposition of incident and reflected waves with a determined duration, the recorded signal
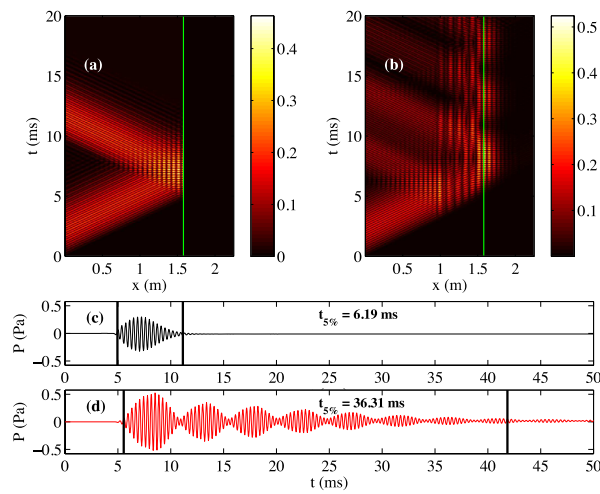

FIG. 5. Figure accounting for the time spreading of the input signal in the chirped sonic crystal. (a), (b) show the time signals recorded for a longitudinal cut in $\mathrm{x}$ direction, for an homogeneous medium (air), and the exponential chirped structure placed in the range $x=[1,2] \mathrm{m}$, respectively. A rigid wall is placed in $x=1.765 \mathrm{~m}$ for the homogeneous case. Green solid lines indicate the position of the time signals shown in (c), (d). Recorded time signals for (c) an homogeneous medium and (d) exponential chirped structure, at $x=1.505 \mathrm{~m}$. 

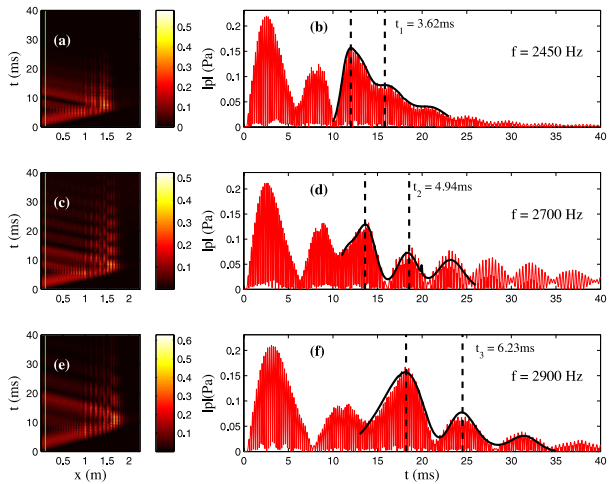

FIG. 6. (a), (c) and (e) Show the time-space maps for 2450, 2700 and $2900 \mathrm{~Hz}$, and (b), (d), (f) the time profiles evaluated at $x=0.1 \mathrm{~m}$. The amplitude for every inset is given by the absolute value of the pressure, $|p|$. Green lines shown in (a), (c), (e) illustrate the corresponding positions of the bright planes for the frequencies 2450,2700 and $2900 \mathrm{~Hz}$, respectively. Black solid lines in insets (b), (d), (f), represent the fitted Gaussian functions used to estimate the delay between consecutive contributions, which are $t_{1}=3.62, t_{2}=4.94$ and $t_{3}=6.23 \mathrm{~ms}$, for 2450,2700 and $2900 \mathrm{~Hz}$, respectively.

for the chirped structure has a longer duration due to the slowing down of the group velocity and it is modulated by the several contributions due to multiple reflections back and forth of the wave inside the chirped crystal. The duration of the whole signal is determined using the criteria that the duration is the time between the instants at which the amplitude is a $5 \%$ of the maximum amplitude of the pulse. This instants are marked in Figs. 5(c) and 5(d) by vertical lines. With this criteria, the time duration of the pulse for the reflection in a rigid wall is $14.5 \mathrm{~ms}$ (see Fig. 5(c)), while the duration of the pulse in the chirped crystal is $40.5 \mathrm{~ms}$ (see Fig. 5(d)). A similar phenomenon of pulse spreading is reported in Ref. 31, where sound diffusers based on biperiodic sonic crystal structures are presented.

Time-space maps for 2450, 2700 and $2900 \mathrm{~Hz}$ and time profiles evaluated at $x=0.1 \mathrm{~m}$ are represented in Fig. 6. As it was previously mentioned, several contributions appear in the upper part of every map $x$ - $t$ corresponding to the successive reflections of the wave packet inside the chirped structure. The wave packet, as it propagates inside the structure, is slowed down and reflected in the turning plane, propagating back to the entrance, but also reflected again in the entrance. This process is repeated until the amplitude of the wave is vanished. This modulation effect by reflections is clearly shown in the signals recorded at $x=0.1 \mathrm{~m}$, shown in Figs. 6(b), (d), and (f), where the amplitude is given by the absolute value of the sound pressure, $|p|$. From the time delay between two different reflections, as we will see immediately, we can obtain information about the slowing down in the crystal. To evaluate this delay between two consecutive reflections from the numerical time domain simulations, time signals are fitted using gaussian functions. ${ }^{32}$ Calculating the distance between the two centroid marked in the same Figs. 6(b), (d) and (f), these values are 3.6, 4.9 and $6.2 \mathrm{~ms}$.

This delay is related to the acoustic path of the pulse inside the chirped structure in which the group velocity gradually changes. Following our approach we can calculate the value of the group velocity in every plane of the structure at each frequency using the corresponding band structures $\left(v_{g}=\partial \omega / \partial k\right.$, Fig. 1), i.e., we are able to evaluate the group velocity profile inside the structure. Table I shows the group velocity values for the three frequencies evaluated in this Section. Taking into account these values and the position of the turning plane for every frequency, we can calculate the delay between two consecutive reflections of the pulse simply by the following expression

$$
t_{f}^{\text {delay }}:=2 \int \frac{d x}{v_{f}(x)}=2 \sum_{i=1}^{N_{f}} \frac{a_{i}}{v_{f, i}} .
$$

where the integral has been replaced by a sum considering that the velocity is kept constant and equal to the value deduced from the band structures that corresponds to the lattice constant $a_{i}$ for 
TABLE I. Values of the group velocity deduced from the band structures calculated with Plane Wave Expansion, for every frequency analyzed and for the different values of the lattice constant $a$, associated to every plane of the chirped structure.

\begin{tabular}{lccc}
\hline \hline Lattice constant & & $v_{g}\left(\mathrm{~ms}^{-1}\right)$ & $2900 \mathrm{~Hz}$ \\
\hline$a(\mathrm{~cm})$ & $2450 \mathrm{~Hz}$ & $2700 \mathrm{~Hz}$ & 89.75 \\
\hline 12,50 & 254.92 & 94.24 & 52.91 \\
11,03 & 292.86 & 278.36 & 284.52 \\
9,87 & 296.37 & 296.37 & 292.49 \\
8,95 & 276.88 & 287.53 & 274.88 \\
8,18 & 196.34 & 265.07 & 245.86 \\
7,54 & 0 & 154.80 & 83.77 \\
6,99 & 0 & 0 & 0 \\
6,52 & 0 & 0 & 2 \\
\hline \hline
\end{tabular}

every crystal plane. The time delay depend on frequency, as it is explicitly indicated in the summation. $N_{f}$ depends on $f$ because this sum extends up to the turning plane which depends on the frequency $f$, because of the chirped. The values obtained applying Eq. (5) are respectively 3.9, 6.3 and $11.1 \mathrm{~ms}$. Although these values are compatible with the ones obtained by the gaussian fitting of the selected peaks in Figs. 6(b), (d) and (f), some differences are found for 2700 and $2900 \mathrm{~Hz}$. These can be explained considering that the difference in the group velocity between air and the first two planes of the chirped structure, according to the values shown in Table I, is very large. A wave packet travelling into a perfectly periodic structure having the lattice constant of either of the two first planes of the chirped structure, $a=12.5$ or $a=11.03 \mathrm{~cm}$, will slow down gradually till the theoretical value of the group velocity is achieved, after travelling through several planes of the structure. Hence, the same wave packet entering the chirped structure will not reach this theoretical value of the group velocity, but slow down slightly before passing the first two planes.

\section{CONCLUSIONS}

Sound enhancement in chirped crystals has been revealed as an efficient mechanism for the wave spatial localization depending on its frequency and on the parameters of the structure. The acoustic wave can be selectively enhanced at particular depth of the crystal, producing bright planes for the frequencies around the first gap along the propagation direction. We reveal here that an exponential chirp can present a stronger enhancement than that produced by a linear chirp, showing an enhancement of 60 times in intensity. A simple couple mode theory is proposed, that captures well the observed effects. For testing these ideas an experimental setup was constructed based on chirped sonic crystal in the audible regime. Measurements of the acoustic field inside of the chirped structure were obtained. On the other hand, numerical calculations using FEM in the spatial and time domain have been performed, showing a perfect agreement with the experimental results. The results and insights from our simple analytical model based on coupled modes theory allow us to keep confidence over the approaches adopted, specially the consideration of our structure as locally periodic. In addition, the reflection from chirped structures was also studied in time domain, by numerical simulations, which show a measurable time spreading of the reflected pulse. The latter result is in accordance with our concept of field slowing down and spatial localization at the turning plane of chirped crystal. Generally speaking, the effect of wave enhancement opens new possibilities for increasing the efficiency of detectors and absorbers, both in acoustics and optics, since slow phonons and photons can be absorbed and harvested with a higher probability.

\section{ACKNOWLEDGMENTS}

The work was supported by Spanish Ministry of Economy and European Union FEDER through project FIS2011-29731-C02-02. LMGR Acknowledges Supported by MINECO and FEDER, under 
Grant MTM2012-36740-c02-02. ACR is grateful for the support of Programa de Ayudas e Iniciativas de Investigación (PAID) of the UPV.

${ }^{1}$ J. D. Joannopoulos, S. G. Johnson, J. N. Winn, and R. D. Meade, Photonic Crystals. Molding the Flow of Light (Princeton University press, 2008).

${ }^{2}$ Y. Pennec, J. O. Vasseur, B. Djafari-Rouhani, L. Dobrzynski, and P. A. Deymier, Surface Science Reports 65, 229 (2010).

${ }^{3}$ M. Kushwaha, P. Halevi, L. Dobrzynski, and B. Djafari-Rouhani, Phys. Rev. Lett. 71, 2022 (1993).

${ }^{4}$ R. Martínez-Sala, J. Sancho, J. V. Sánchez, V. Gómez, J. Llinares, and F. Meseguer, Nature 378, 241 (1995)

${ }^{5}$ X. Zhang and Z. Liu, Appl. Phys. Lett 85, 341 (2004).

${ }^{6}$ M.-H. Lu, C. Zhang, L. Feng, J. Zhao, Y.-F. Chen, Y.-W. Mao, Y.-Y. Zhu, S.-N. Zhu, and N.-B. Ming, Nature Mat. 6, 744 (2007).

${ }^{7}$ I. Pérez-Arjona, V. J. Sánchez-Morcillo, J. Redondo, V. Espinosa, and K. Staliunas, Phys. Rev. B 75, 014304 (2007).

${ }^{8}$ V. Espinosa, V. J. Sánchez-Morcillo, K. Staliunas, I. Pérez-Arjona, and J. Redondo, Phys. Rev. B 76, 140302(R) (2007).

${ }^{9}$ Y. Zhou, M.-H. Lu, L. Feng, X. Ni, Y.-F. Chen, Y.-Y. Zhu, S.-N. Zhu, and N.-B. Ming, Phys. Rev. Lett. 104, 164301 (2010).

${ }^{10}$ V. Sánchez-Morcillo, R. Picó, I. Pérez-Arjona, and K. Staliunas, Applied Acoustics 73, 302 (2012).

${ }^{11}$ R. Picó, V. Sánchez-Morcillo, I. Pérez-Arjona, and K. Staliunas, Appl. Acoust. 73, 302 (2012).

12 A. Khelif, P. A. Deymier, B. Djafari-Rouhani, J. O. Vasseur, and L. Dobrzynski, J. Appl. Phys. 94, 1308 (2003).

${ }^{13}$ F. Cervera, L. Sánchis, J. Sánchez-Pérez, R. Martínez-Sala, C. Rubio, and F. Meseguer, Phys. Rev Lett. 88, 023902 (2002).

${ }^{14}$ A. Cebrecos, V. Romero-García, R. Picó, I. Pérez-Arjona, V. Espinosa, V. Sánchez-Morcillo, and K. Staliunas, J. Appl. Phys. 111, 104910 (2012)

${ }^{15}$ X. Li, X. Ni, L. Feng, M. Lu, C. He, and Y. Chen, Phys. Rev. Lett. 106, 084301 (2011).

16 V. Romero-García, J. Sánchez-Pérez, S. Castiñeira Ibáñez, and L. Garcia-Raffi, Appl. Phys. Lett. 96, 124102 (2010).

${ }^{17}$ V. Romero-García, J. Sánchez-Pérez, and L. Garcia-Raffi, J. Appl. Phys. 108, 044907 (2010).

${ }^{18}$ H. Hu, A. Strybulevych, J. H. Page, S. E. Skipetrov, and B. A. van Tiggelen, Nature Physics 4, 945 (2008).

${ }^{19}$ R. Sainidou, N. Stefanou, and A. Modinos, Phys. Rev. Lett. 94, 205503 (2005).

${ }^{20}$ V. Romero-García, R. Picó, A. Cebrecos, V. Sánchez-Morcillo, and K. Staliunas, Appl. Phys. Lett. 102, 091906 (2013)

${ }^{21}$ E. Cassan, C. K.-V. D. Caer, D. Marris-Morini, and L. Vivien, J. Lightwave Tech. 29, 1937 (2011).

${ }^{22}$ Y. Cheng, S. Kicas, J. Trull, M. Peckus, C. Cojocaru, R. Vilaseca, R. Drazdys, and K. Staliunas, Sci. Rep. 4, 6326 (2014).

${ }^{23}$ M. Kushwaha, B. Djafari-Rouhani, L. Dobrynski, and J. Vasseur, Eur. Phys. J. B 3, 155 (1998).

${ }^{24}$ I. E. Psarobas and M. M. Sigalas, Phys. Rev. B 66, 052302 (2002).

${ }^{25}$ L. Wu and L. Chen, J. Appl. Phys. 110, 114507 (2011).

${ }^{26}$ Y. Shen, J. Fu, and G. Yu, Phys. Lett. A 375, 3801 (2011).

${ }^{27}$ M. Stockman, Phys. Rev. Lett. 93, 137404 (2004).

${ }^{28}$ V. N. Smolyaninova, I. I. Smolyaninov, A. V. Kildishev, and V. M. Shalaev, Appl. Phys. Lett. 96, 211121 (2010).

${ }^{29}$ E. Centeno, D. Cassagne, and J.-P. Albert, Phys. Rev. B 73, 235119 (2006).

${ }^{30} \mathrm{http}$ ://functions.wolfram.com/HypergeometricFunctions/LaguerreL3General/.

31 J. Redondo, R. Picó, V. J. Sánchez-Morcillo, and W. Woszczyk, J. Acoust. Soc. Am. 134, 4412 (2013).

${ }^{32}$ A. Cicek, O. A. Kaya, M. Yilmaz, and B. Ulug, J. Appl. Phys. 111, 013522 (2012). 


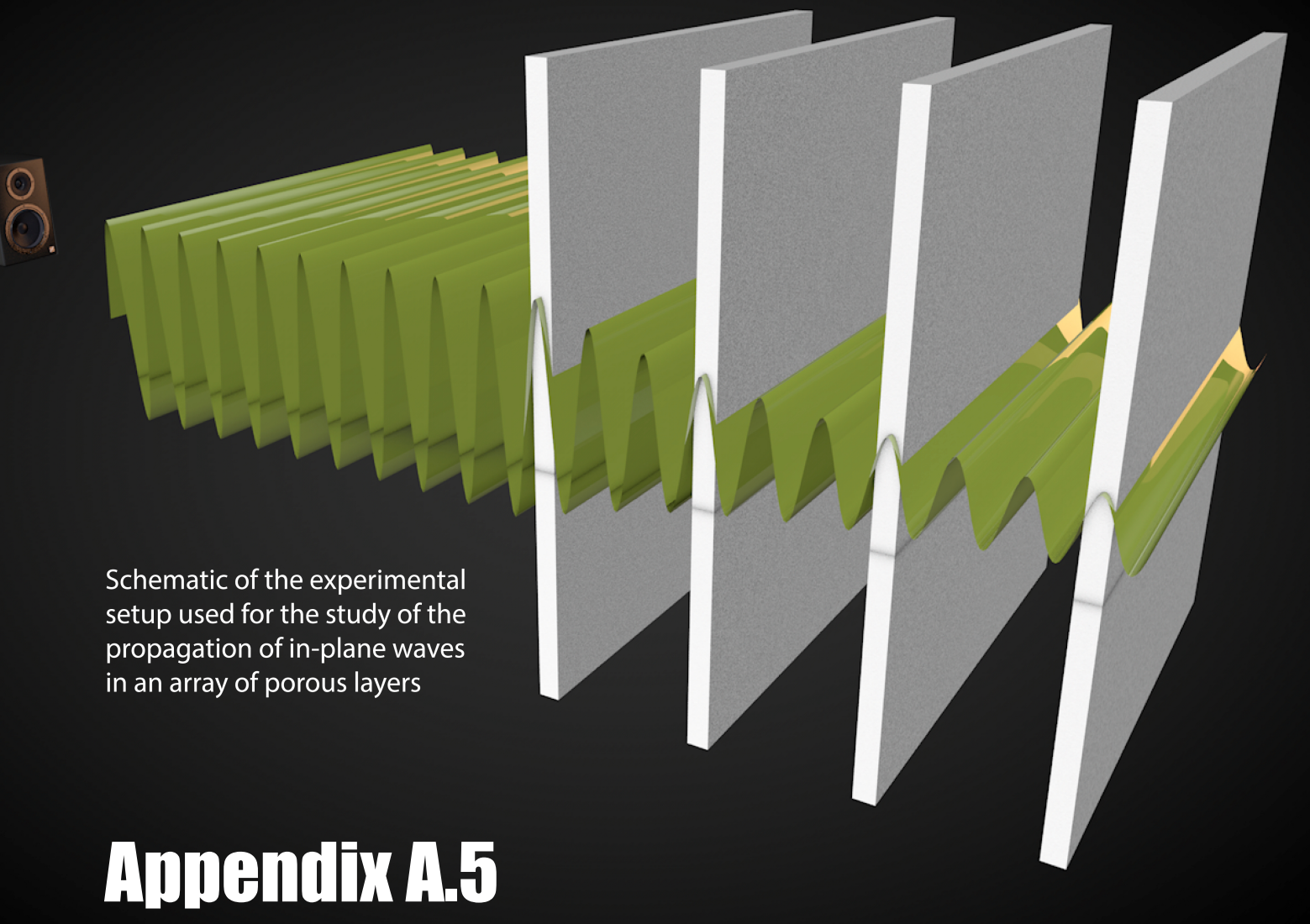

\section{Enhanced Transmission Band in Periodic Media with Loss Modulation}

This paper is devoted to the propagation of in-plane waves in a periodic array of absorbing layers. An anomalous increase of wave transmission through the structure, related to a decrease in the absorption around the Bragg frequencies, is found. The coupled mode theory, used in previous works, is extended here to include the effect of losses, allowing to predict and discuss this intriguing phenomena. Experimental measurements are carried out for the case of sound waves in an array of porous layers embedded in air, demonstrating the existence of an enhanced transmission band. The author of this thesis participated in numerical simulations and experimental measurements. 


\title{
Enhanced transmission band in periodic media with loss modulation
}

\author{
A. Cebrecos, ${ }^{1}$ R. Picó, ${ }^{1}$ V. Romero-García, ${ }^{2}$ A.M. Yasser, ${ }^{1,3}$ L. Maigyte, ${ }^{4}$ R. Herrero, ${ }^{4}$ M. Botey, ${ }^{4}$ V. J. \\ Sánchez-Morcillo, ${ }^{1}$ and K. Staliunas ${ }^{4,5}$ \\ ${ }^{1)}$ Instituto de Investigación para la Gestión, Integrada de las Zonas Costeras, \\ Universitat Politècnica de València, c/ Paranimf 1, 46730, Grao de Gandia, \\ Spain \\ ${ }^{2)}$ LUNAM Université, Université du Maine, CNRS, LAUM UMR 6613, Av. O.Messiaen, 72085 Le Mans, \\ France \\ ${ }^{3)}$ Physics Department, Faculty of Science at Qena, South Valley University, \\ Egypt \\ ${ }^{4)}$ Department de Física i Enginyeria Nuclear, Universitat Politècnica de Catalunya, Rambla de Sant Nebridi 22, \\ 08222 Terrasa, Spain \\ ${ }^{5)}$ Institució Catalana de Recerca i Estudis Avanats (ICREA), Colom 11, 08222 Terrasa, Barcelona, \\ Spain.
}

We study the propagation of waves in a periodic array of absorbing layers. We report an anomalous increase of wave transmission through the structure related to a decrease of the absorption around the Bragg frequencies. The effect is first discussed in terms of a generic coupled wave model extended to include losses, and its predictions can be applied to different types of waves propagating in media with periodic modulation of the losses at the wavelength scale. The particular case of sound waves in an array of porous layers embedded in air is considered. An experiment designed to test the predictions demonstrates the existence of the enhanced transmission band.

PACS numbers: 43.20.Hq, 43.20.Fn, 42.25 Bs, 78.67 Pt

Keywords: Periodic media, Layered lossy media, Band gap materials

Wave propagation in periodic media has become a subject of intensive study with numerous applications in different fields. The simplest form of periodic media consist of alternating material layers with different properties (such as the refraction index in optics, or the density or elasticity parameters in acoustics) forming a layered medium, also referred as $1 \mathrm{D}$ crystal or superlattice. Originally formulated to explain the propagation of electrons in solids ${ }^{1}$, the basic theory of wave propagation in layered media was soon extended to optics ${ }^{2}$ and acoustics ${ }^{3,4}$. Most of the previous work on periodic media focused on conservative systems where waves can be reflected (at the bandgaps), deflected, scattered, or even localized inside the crystal. However, waves cannot be absorbed unless dissipation is considered in the system. While dissipation is an inherent property of all forms of matter, few attention has been paid to its effects in periodic media. Moreover, especially in real experiments, often one or more of the constituent materials present some non-negligible losses in the frequency range of interest.

Light and sound waves behave in the same manner in linear media, obeying similar wave equations. This has inspired a number of analogies between both fields. However, the motivation for the study of losses in acoustics and optics may be different. In optics, where efforts are devoted to minimize losses, dissipation in periodic systems has been considered recently ${ }^{5-10}$. While in Refs. [5] and [6] absorption is reduced in a multilayered magneto-photonic crystal, in Refs. [7] and [8] enhanced transmission through a stack of dielectric layers having contrast only in attenuation is reported. Extensions to two-dimensional (2D) modulation of losses has shown to provide nontrivial light beam propagation effects, analo- gous to flat photonic crystal lensing reported in conservative systems ${ }^{9,10}$. In acoustics the situation is different, since achieving maximum absorption is often the goal. The effect of viscoelastic losses on phononic crystals was first discussed in Ref. [11], and more recently in Refs. [12-14], in terms of the modification of dispersion relations. Damping of elastic waves in solids phononic crystals has also been discussed in [15] and [16]. In the audible regime, viscothermal losses dominate, and absorption is mainly achieved by using resonators or porous materials ${ }^{17}$. The behaviour of lossy periodic media for waves near Bragg resonances is much less known than the long-wavelength limit. In this regime, there are studies about wave propagation in acoustic absorbing media with rigid periodic inclusions ${ }^{18}$, and in $2 \mathrm{D}$ arrays made of absorbent ${ }^{19}$ and, absorbent and resonant scatterers embedded in air ${ }^{20}$. The combination of periodicity and absorption in substructured materials produces complete absorption of sound with a broadband response and functional for any direction of incident radiation ${ }^{21}$.

In this work we investigate the wave propagation within a layered material with periodically distributed losses. We show how the periodicity of the absorbing media can modify the global absorption of the system as well as its reflection and transmission properties. The main prediction is a simultaneous increase of transmission and reflection around the Bragg frequency, an anomalous behavior in contrast to classical, conservative bandgaps that always result in a decrease of transmission. First, a generic model based on the coupled-mode theory and valid for different types of waves (light, sound or matter waves) and media is presented, and its transmission/reflection characteristics are analytically deter- 

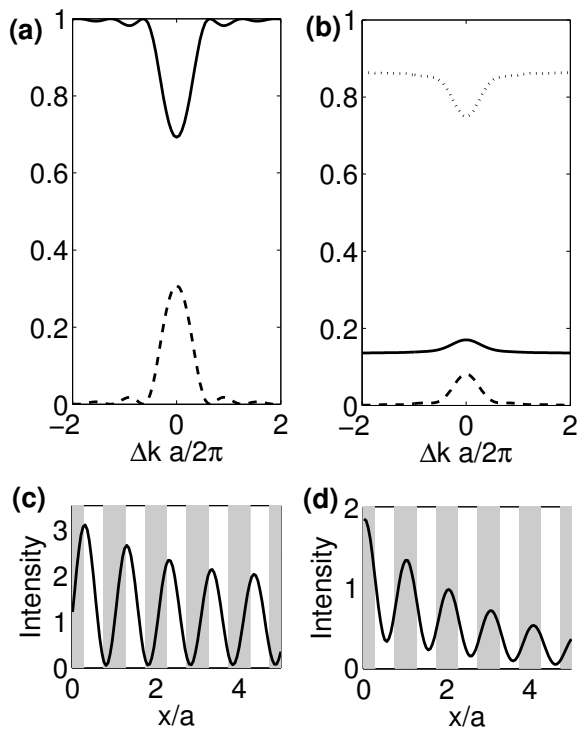

FIG. 1. Transmission (solid lines) reflection (black dotted lines) and absorption (gray dotted lines) spectra for waves in a periodic structure ( 5 periods, $L / a=5$ ) as calculated from Eqs. (3) and (4) for (a) conservative system (with coupling $m a=0.125$ and no losses $\gamma a=0)$ showing the well known band-gaps, (b) periodic system (with pure imaginary coupling valued $m a=i 0.125$ and losses $\gamma a=-0.2)$ predicting the anomalous transmission. (c) and (d) show the total intensity at the Bragg frequency, $\Delta k=0$, for the configurations shown in (a) and (b) respectively. Grey areas represent the absorbing material in (d).

mined. Next, we particularize the study to the case of sound waves propagating in a $1 \mathrm{D}$ periodic structure of porous layers embedded in air, which is theoretically and experimentally examined. The anomalous of transmission band around bandgap frequencies is experimentally observed, showing good agreement with theory even for a minimal number of layers.

Waves in layered media can be studied by using different theoretical tools. One approach very popular in photonics is the coupled-mode theory ${ }^{22}$. Here we extend the theory to include the effect of losses, and calculate its influence in the transmission/reflection spectrum. Consider a medium formed by a finite number of lossy parallel identical and equidistant layers irradiated by an incident plane wave. The total field is composed of forward and backward propagating waves $P=A(x) e^{i k_{B} x-i \omega t}+B(x) e^{-i k_{B} x-i \omega t}+c . c$. , which amplitudes are normalized so that their absolute square is proportional to the energy flux in the corresponding direction. $k_{B}=\pi / a$ is the Bragg wavenumber (the edge of Brillouin zone, being $a$ the lattice constant of the system) and $\omega$ is the frequency. Forward and backward waves are coupled by the modulation. If the contrast of impedances between layers is small, and for frequencies near a Bragg resonance, the dynamics of the forward and backward waves can be approximately described by the dissipative coupled-mode equations,

$$
\begin{aligned}
\frac{d A}{d x} & =i \Delta k A+m B+\gamma A, \\
-\frac{d B}{d x} & =i \Delta k B+m A+\gamma B,
\end{aligned}
$$

where $\Delta k=k-k_{B}$ is the detuning from the Bragg wavenumber, $m$ is the coupling between forward and backward waves which is generally complex: real for reflections from conservative (rigid or penetrable without losses) materials, and imaginary for reflections from purely absorptive media. A complex value of $m$ allows representing any realistic material. The coupling coefficient $m$ is related to the impedance mismatch between the absorber and the host medium. If the reflection coefficient from medium 1 to medium 2 is $r_{12}$ and $r_{21}=-r_{12}$, and considering the same acoustic thickness (or equivalently, the optical path) $d$ for both materials, the coupling coefficient is: $m=\left(r_{12}-r_{21}\right) / d=2 r_{12} / d$. For the case of an acoustic wave: $r_{12}=\left(Z_{2}-Z_{1}\right) /\left(Z_{2}+Z_{1}\right)$, where $Z_{i}$ stands for the impedance of the $i$-th medium. Finally $\gamma$ is the gain coefficient, being negative for the case of a lossy media. Its worth noting that $\gamma$ is always negative for an acoustic media $(\gamma<0$, since there are no gain acoustic materials). Note that the following relation holds $|\gamma|>|\operatorname{Im}(m)|$.

The solutions of Eqs. (1) are exponentially growing/decaying oscillating waves, $A(x), B(x)=e^{\lambda x}$, where $\lambda$ are the complex eigenvalues of the matrix of the coefficients of Eqs. (1), which read

$$
\lambda_{ \pm}= \pm \sqrt{(\gamma+i \Delta k)^{2}+m^{2}} .
$$

For a finite system of length $L$, formed by $N$ layers, transmission and reflection coefficients can be obtained analytically by imposing boundary conditions at the entrance face $(x=0)$ for the forward field, $A(x=0)=0$, and at the rear face $(x=L)$ for the backward field $B(x=L)=0$. This leads to

$$
\begin{aligned}
T & =\frac{\lambda}{\lambda \cosh (\lambda L)-(\gamma+i \Delta k) \sinh (\lambda L)} \\
R & =\frac{m \sinh (\lambda L)}{\lambda \cosh (\lambda L)-(\gamma+i \Delta k) \sinh (\lambda L)}
\end{aligned}
$$

with $\lambda$ given by Eq. (2) with the negative sign (physical solutions of the problem).

These expressions can be used to evaluate the response of the structure in two opposite cases: the well-known conservative periodic system $\gamma=0$ and pure real modulations parameter and a fictional material called here purely absorptive material, that is a medium with the same real part of the impedance as the host, but a nonnull imaginary part, i.e. pure imaginary $m$ and negative $\gamma$. The latter case is analogous to that considered 
for photonics in Refs. [7] and [8]. As it is well known, for conservative periodic materials, the waves around the Bragg frequency $f_{B}=c / 2 a$ (being $c$ the velocity of the wave in the medium) are efficiently back reflected due to Bragg resonance and transmission is correspondingly reduced, as shown in Fig. 1(a).

However, in the case of lossy periodic media, the situation is different since the material parameters may have a complex value due to dissipation. In the ideal case of a purely absorbent material, we observe that an anomalous transmission is maximum at Bragg resonance $(\Delta k=0)$, as observed in Fig. 1(b). The origin of such anomalous phenomenon is explained in Figs. 1(c) and 1(d), where the field distribution along the structure is shown for both cases. For a purely absorbent structured material, at these frequencies, the total field within the structure partially forms a standing wave, with the nodes of the particle velocity (maximum values of the field) located precisely inside the absorbing media. As the nodes correspond to low particle velocity, there is few energy to be absorbed. As a consequence, such a configuration results in smaller absorption: both forward as well as backward waves are less absorbed, and the overall transmission is increased as well as the absorption is reduced as shown in Fig. 1(b).
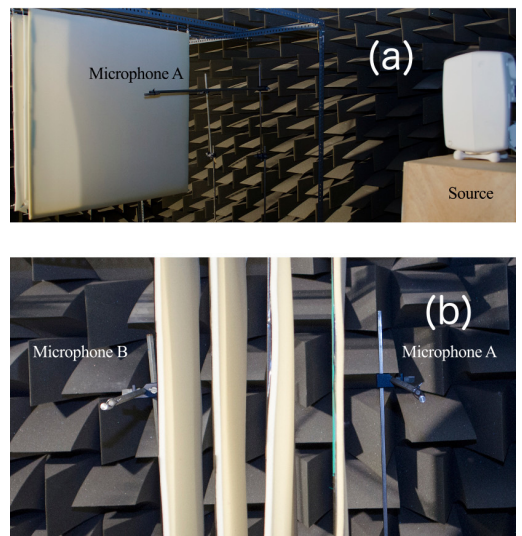

FIG. 2. (Color online) (a) Experimental set-up, consisting in an array of four plates of porous material; showing the source, a loudspeaker located in front of the structure, and the microphone to measure intensity at either side of the structure. (b) View of the system from a different angle.

The coupled wave formulation presented above is independent of the particular type of wave. Then, the coefficients are generic and do not contain information on the physical characteristics of the considered system. We concentrate now in the particular case of sound waves propagating through periodically spaced porous layers, of thickness $D$ embedded in a fluid media (air) being $a$ the distance between the center of two consecutive layers (lattice constant). This study will be used to check the predictions of the general model as well as to compare with experiments.

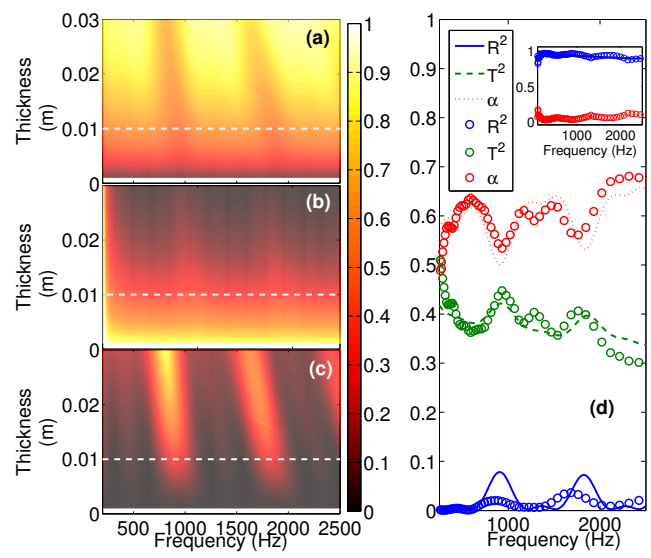

FIG. 3. (Color online) (a), (b) and (c) Dependence of the absorption, reflection and transmission coefficients on the thickness of the porous layer and on the frequency for the stratified media, calculated with TMM for $N=3$ layers. (b) Reflection (blue continuous), Transmission (green dashed) and Absorption (red dotted) of our system (corresponding to the white dashed line in (a)-(c)). Continuous lines represent the theoretical predictions and circles represent the experimental results. Inset shows the reflection (blue continuous) and absorption (red dotted) coefficients of a single porous layer for its characterization using the ISO-10534-2.

An experiment was designed to check the predictions of anomalous transmission around Bragg frequencies. The set-up consists of a set of 3 to 5 parallel porous layers of $D=8 \mathrm{~mm}$ thickness embedded in air, as shown in Fig. (2). The lattice constant was chosen as $a=20 \mathrm{~cm}$. A loudspeaker was placed in front of the first layer in such a way that plane waves propagate through the system. All the measurements were conducted in an anechoic chamber in order to avoid unwanted reflections. The coefficients (reflection, transmission and absorption) were calculated from the acoustic pressure measurements registered by two microphones, in both sides of the periodic structure. The spectral characteristics were measured using the above described experimental scheme. Experimentally, we determined the intensity coefficients by measuring the sound field before (reflection $R$ ) and after (transmission $T$ ) the structure. Finally, by energy balance, the absorption coefficient is obtained as $\alpha=1-|R|^{2}-|T|^{2}$.

We consider here the most general case in which the frame of the porous material presents an elastic behaviour, so Biot's theory can be used to characterize the porous material. The layered material used in experiments is analytically characterized by the transfer matrix method (TMM) described in Ref. [17]. We consider that the layered structure is laterally infinite (1D) and 
made of homogeneous and isotropic porous layers embedded in air. We calculate the transfer matrices in the porous medium where two compressional waves and one shear wave can be supported and in the fluid medium with only one compressional wave. All these waves are coupled by the boundary conditions and the result is a global transfer matrix which gives the propagation properties of the stratified media made of $N$ layers, and in particular its reflection and transmission coefficients.

\begin{tabular}{cc}
\hline \hline Densitiy $\left(\mathrm{kg} / \mathrm{m}^{3}\right), \rho$ & 50 \\
\hline Porosity, $\phi$ & 0.97 \\
\hline Young's Modulus (kPa), $E$ & 150 \\
\hline Poisson's coefficient & 0.35 \\
\hline Tortuosity, $\alpha_{\infty}$ & 1 \\
\hline Flow resistivity, $\sigma$ & 13000 \\
\hline Characterisitic length $(\mathrm{m}), \Lambda$ & $120 \times 10^{-6}$ \\
\hline Characterisitic thermal length $(\mathrm{m}), \Lambda^{\prime}$ & $200 \times 10^{-6}$ \\
\hline
\end{tabular}

TABLE I. Physical parameters of the porous material used in the experiments and numerics.

In a first step, the material has been characterized. Parameters of the material are shown in Tab. I. These parameters have been used to evaluate the transmission and absorption coefficients of the porous layer using the TMM. These properties are shown in the inset of Fig. $3(\mathrm{~d})$, showing that the parameters of Tab. I represents in good agreement the transmission and absorption properties obtained using the standard ISO-10534-2. We can see that the absorption of the porous material is very low, therefore the effective impedance of the porous layer is similar to that of the air. This situation is optimal to allow transmission with small but enough losses to induce the anomalous properties of a layered media made of layers of this porous material.

Once the material is characterized, we use the TMM to evaluate the properties of a layered material made of 3 porous layers embedded in air. The dependence of the absorption, $\alpha$, reflection, $|R|^{2}$, and transmission, $|T|^{2}$, coefficients on the thickness of the porous layer, $d$, and on the frequency, $f$, are shown in Figs. 3(a), 3(b) and 3(c) respectively. We can observe, as predicted previously by the general coupled-mode model, the usual increase of the reflection in the band-gap and the anomalous increase (decrease) of the transmission (absorption) at frequencies around the band gap $\left(f_{B}=850 \mathrm{~Hz}\left(f_{B}=1700 \mathrm{~Hz}\right)\right.$ for the first (second) band gap).

Finally, we particularize for the case we have in the experimental set-up. Figure 3(d) shows the comparison between the numerical predictions, obtained by applying the TMM and the experimental results. As predicted, maxima of transmission and reflection are observed at Bragg frequencies and, as a consequence, at these frequencies the structure is absorbing less energy.

We determine transmission and reflection of waves in a general layered lossy structure and measure it experi- mentally in a particular acoustic system. The study indicates the existence of spectral regions of enhanced and reduced overall absorption with anomalous transmission around the band gap. A simple couple mode theory is proposed to explain these results, which is essentially a forward wave linearly coupled with the backward wave. Depending on the character of the systems (rigid, lossy, or complex), the coupling coefficient is set (real, imaginary or complex), which also captures the above predicted and measured spectral characteristics. In good agreement with the TMM predictions, we experimentally observe that the transmission of sound waves trough a periodic arrangement of absorbing plates is enhanced at resonance. Such anti-bandgap effect is expected to be generic for any kind of waves in a periodic modulation of losses on the wavelength scale, at the Bragg frequency.

The work was supported by Spanish Ministry of Science and Innovation and European Union FEDER through projects FIS2011-29731-C02-01 and -02, also MAT2009-09438. A. M. Y. would like to thank the Erasmus Mundus project (WELCOME program) for supporting him.

${ }^{1}$ Brillouin L., Wave Propagation in Periodic Structures. Dover, New York (1953).

${ }^{2}$ Pochi Yeh, Optical Waves in Layered Media, Wiley (2005)

${ }^{3}$ Brekhovskikh, L.M., 1960. Waves in Layered Media. Academic Press, New York.

${ }^{4}$ L. M. Brekhovskikh, O. A. Godin, Acoustics of Layered Media I: Plane and Quasi Plane Waves, Springer Verlag, New York, (1998).

${ }^{5}$ A. Figotin, I. Vitebskiy, Phys. Rev. B 77, 104421 (2008)

${ }^{6}$ A. Figotin, I. Vitebskiy, Waves in Random and Complex Media 20, 298-318 (2010)

${ }^{7}$ S.G. Erokhin, A.A. Lisyansky, A.M. Merzlikin, A.P. Vinogradov and A.B. Granovsky, Phys. Rev. B 77, 233102 (2008).

${ }^{8}$ N. Kumar, M. Botey, R. Herrero, Y. Loiko, K. Staliunas, Photonics and Nanostructures-Fundamentals and Applications 10, 4, 644-650 (2012).

${ }^{9}$ K. Staliunas, R. Herrero, and R. Vilaseca, Phys. Rev. A 80 , 013821 (2009)

${ }^{10}$ N. Kumar, R. Herrero, M. Botey and K. Staliunas, J. Opt. Soc. Am. B 30, 2684-2688 (2013).

${ }^{11}$ I.E. Psarobas, Phys. Rev. B 64, 012303, (2001).

${ }^{12}$ C.Y. Lee, M.J. Leamy and J.H. Nadler, J. Sound Vib. 329, 1809 (2010).

${ }^{13}$ V. Laude, J.M. Escalante and A. Martinez, Phys. Rev. B 88 , $224302(2013)$

${ }^{14}$ J. H. Oh, Yoon J. Kim, Y. Y. Kim, J. Appl. Phys. 113, 106101 (2013).

${ }^{15}$ M.I. Hussein, Phys. Rev. B 80, 212301 (2009)

${ }^{16}$ E. Andreassen, J. S. Jensen, J. Vib. Acoust. 135, 041015 (2013)

${ }^{17} \mathrm{~J}$. Allard, N. Atalla, Propagation of sound in porous: Modeling sound absorbing materials, John Wiley and Sons (2009).

${ }^{18}$ V. Tournat, V. Pagneux, D. Lafarge, L. Jaouen, Phys. Rev. E, 70, $026609(2004)$

${ }^{19}$ O. Umnova, K. Attenborough, C.M. Linton, J. Acoust. Soc. Am 119, 278-284 (2006)

${ }^{20}$ V. Romero-García, J. V. Sánchez-Pérez, L.M. Garcia-Raffi, J. Appl. Phys. 108, 044907 (2010)

${ }^{21}$ J. Christensen, V. Romero-García, R. Picó, A. Cebrecos, F. J. García de Abajo, N. A. Mortensen, M. Willatzen and V. J. Sánchez-Morcillo, Sci. Rep. 4, 4674 (2014).

${ }^{22}$ H. Kogelnik and C. V. Shank, J. Appl. Phys. 43, 23272335 (1972). 

porous lamella-crystals

\author{
SUBJECT AREAS: \\ FLUID DYNAMICS \\ FLUIDICS \\ J. Christensen 1,2, V. Romero-García ${ }^{3,4}$, R. Picó 3 , A. Cebrecos 3 , F. J. García de Abajo 5,6 , N. A. Mortensen², \\ M. Willatzen ${ }^{2}$ \& V. J. Sánchez-Morcillo ${ }^{3}$
}

Received

19 March 2014

'Institute of Technology and Innovation, University of Southern Denmark, DK-5230 Odense, Denmark, ${ }^{2}$ Department of Photonics Engineering, Technical University of Denmark, DK-2800 Kgs. Lyngby, Denmark, ${ }^{3}$ Instituto de Investigación para la Gestión Integrada de zonas Costeras, Universitat Politècnica de València, Paranimf 1, 46730, Gandia, Spain, 'LUNAM Université,

Accepted

26 March 2014

Published

14 April 2014

Correspondence and requests for materials should be addressed to

J.C. (jochri@fotonik. dtu.dk) Université du Maine, CNRS, LAUM UMR 6613, Av. O. Messiaen, 72085 Le Mans, France, ${ }^{5}$ ICFO Institut de Ciències Fotòniques, Mediterranean Technology Park, 08860 Castelldefels (Barcelona), Spain, ${ }^{\circ}$ ICREA - Institució Catalana de Reserca i Estudis Avançats, Passeig Lluís Companys 23, 08010 (Barcelona), Spain.

We present the design of a structured material supporting complete absorption of sound with a broadband response and functional for any direction of incident radiation. The structure which is fabricated out of porous lamellas is arranged into a low-density crystal and backed by a reflecting support. Experimental measurements show that strong all-angle sound absorption with almost zero reflectance takes place for a frequency range exceeding two octaves. We demonstrate that lowering the crystal filling fraction increases the wave interaction time and is responsible for the enhancement of intrinsic material dissipation, making the system more absorptive with less material.

$T$ he damping of sound waves can be understood by conversion of the mechanical energy into heat. The acoustic equivalent to an ideal black body would be something similar to a "deaf" body which is an object absorbing sound coming from all directions at any given frequency. Absorption of sound waves is governed by the effects of viscosity and thermal conduction in fluids. In order to give a macroscopic picture of these dissipative processes one must interchange the mass density $\rho$ and the bulk modulus $K$ with complex quantities, leading to a finite penetration length into the dissipative medium. In a first approximation, damping can be neglected for audible sound in free space and therefore the utilization of lossy materials is essential for screening noise and designing acoustic insulators. Thus, it is desirable to create structures with the capability of efficient dissipation, preferably in a way that energy conversion causes all waves to be absorbed such that no back- and through-radiation takes place. While this remains to be the ideal case, various composites and artificial structures were designed in the attempt of pursuing this ultimate goal. We distinguish between resonance-based and broadband systems. Locally resonating materials have been fabricated in the form of mass-loaded thin membranes, gas-bubble arrays and elastic beams, featuring sharp and narrow absorption peaks ${ }^{1-5}$. Broadband absorption, on the other hand, has been demonstrated for low frequencies by lattices of perforated shells ${ }^{6}$. Sound blocking screens made out of, e.g., phononic crystal or metamaterials with one single negative effective parameter prevent waves to penetrate through these structures resulting in full reflection of sound ${ }^{7-10}$. These effects are caused by diffraction properties and evanescent modes, respectively, and will not easily lead to broadband absorption of sound. Periodic penetrable structures, generally speaking, have been fabricated with many facets for different kinds of waves. Electromagnetic (EM) structured materials, in that regard, such as gratings with finite conductance, convex grooves or the moth eyes, comprise anti-reflective systems with a broad spectral response $^{11-15}$. However, to sustain a spectrally and angularly rich performance with complete absorption and little material use remains a challenge worth pursuing.

We present a system inspired by recent EM experiments where a forest of vertically aligned single-walled carbon nanotubes showed extremely low reflectance and making it the darkest man-made material ever ${ }^{16-20}$. The mechanism consists in the attempt of matching the material index to free-space to prevent back-reflected waves but at the same time providing sufficient material losses to guarantee intensity attenuation. Here, we show how a low-density porous lamella array, in analogy to its electromagnetic counterpart made of nanotube arrays, behaves most closely like a true deaf body. Within this framework, we show how these constructed crystals in a most counter intuitive way become more absorptive when less material is chosen. 


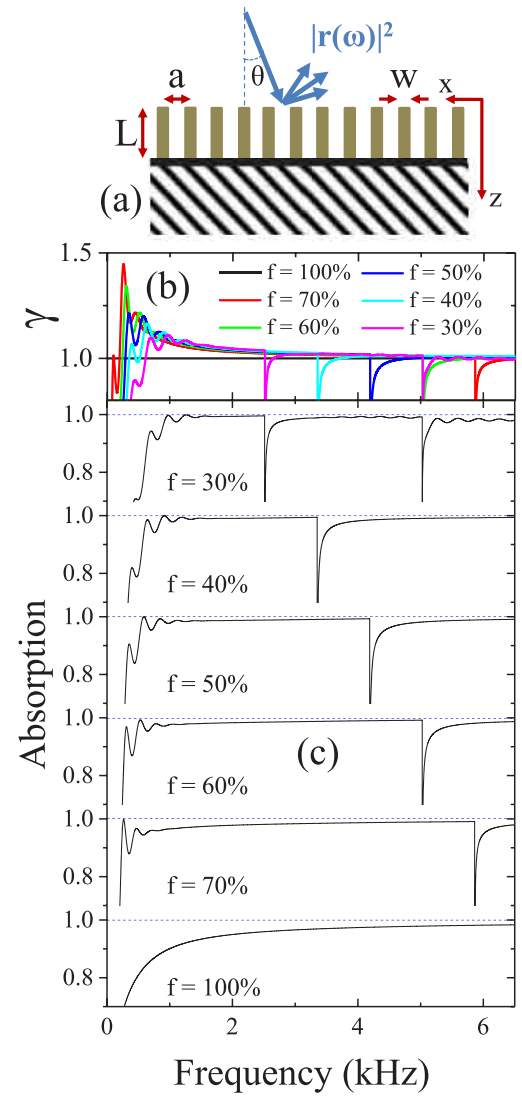

Figure $1 \mid$ Controlling the absorption by lowering the crystal filling fraction $\mathrm{f}$. (a), Schematic of the crystal made of lamellas of width $\mathrm{W}$, lattice constant $a$ and length $L$. The crystal is backed by a rigid support into which no sound waves penetrate. (b,c), The enhancement factor $\gamma$ and the absorption are plotted for various filling fractions with constant lamella width $\mathrm{W}=4 \mathrm{~cm}$ and length $L=0.5 \mathrm{~m}$. In all cases, we implemented complex dispersive material dependence for $\rho$ and $K$ within the lamellas ${ }^{22}$.

\section{Results}

Concept of enhanced absorption in lossy lamella-crystals. To illustrate this surprising behaviour, we begin by analysing a simple asymmetric system consisting of an inhomogeneous lossy medium of length $L$ supported by a rigid backing as illustrated in Fig. 1a. The general complex scattering matrix can be written as:

$$
S(\omega)=\left[\begin{array}{cc}
r(\omega) & 0 \\
0 & 1
\end{array}\right],
$$

where $r(\omega)$ is the complex reflection coefficient. In the absence of losses the scattering matrix is unitary and consequently $|r(\omega)|=1$, i.e. perfect reflection due to the rigid backing. This changes dramatically in the presence of weak absorption provided that the time delay is comparable to $1 / 2 \Gamma$. To see this explicitly, we follow considerations developed in the context of absorption in chaotic cavities $^{21}$. For a weak absorption rate $\Gamma$ the full scattering matrix $S$ is related to its loss-less counterpart $S_{0}$

$$
S(\omega)=S_{0}(\omega)\left(1-\frac{\Gamma}{2} Q(\omega)\right),
$$
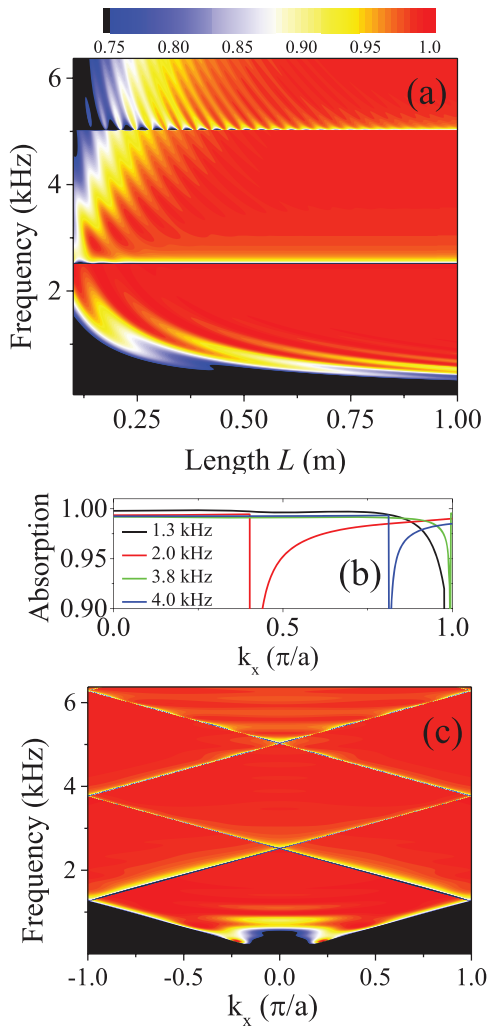

Figure $2 \mid$ Acoustic response of crystals made out of porous lamellas. (a), Absorption versus frequency and lamella length $L$ for normal incident radiation where $\mathrm{f}=30 \%$ and $\mathrm{W}=4 \mathrm{~cm}$. (b,c) Simulated angular dependence of the structure in (a) with $L=0.5 \mathrm{~m}$ within the first Brillouin zone. We take illustrative examples corresponding to four frequency cuts in (c) and show the dependence of absorption with parallel momentum $k_{x}$

where $Q(\omega)=-i S_{0}^{\dagger}(\omega) \frac{\partial S_{0}(\omega)}{\partial \omega}$ is the Wigner-Smith delay-time matrix. Since $S_{0}$ is Hermitian so is $Q$ and its positive eigenvalues are interpreted as the group delay time $\tau$ for a wavepacket centered around $\omega$. Conservation of energy in the asymmetric system requires $|r(\omega)|^{2}+A=1$, where $A$ is the absorption, hence, to a first approximation we can write

$$
|r(\omega)|^{2}=1-2 \Gamma \tau .
$$

Provided a sufficiently long time delay, the reflectance can be minimal and the absorption perfect, even for a modest intrinsic absorption rate. The perspective of acoustic functional materials is to support delay times much exceeding the delay time of their bulk counterparts $\left(\tau \gg \tau_{l}\right)$. For a bulk material lamella $(l)$ of length $L$, backed by a perfect reflector, the acoustical path becomes $2 L$ and consequently the time delay is $\tau_{l}=2 L / c_{l}$ where $c_{l}$ is the sound speed in the lamella material. On the other hand, composing a porous periodic crystal from the same base material, $c_{l}$ is replaced by the actual group velocity $v_{g}$ associated with the phononic band dispersion relation. In this context, we define the enhancement factor $\gamma=\frac{\Gamma \tau}{\Gamma_{l} \tau_{l}}$ that expresses the acoustic interaction strength in the crystal. In other words, enhancement is reached whenever 

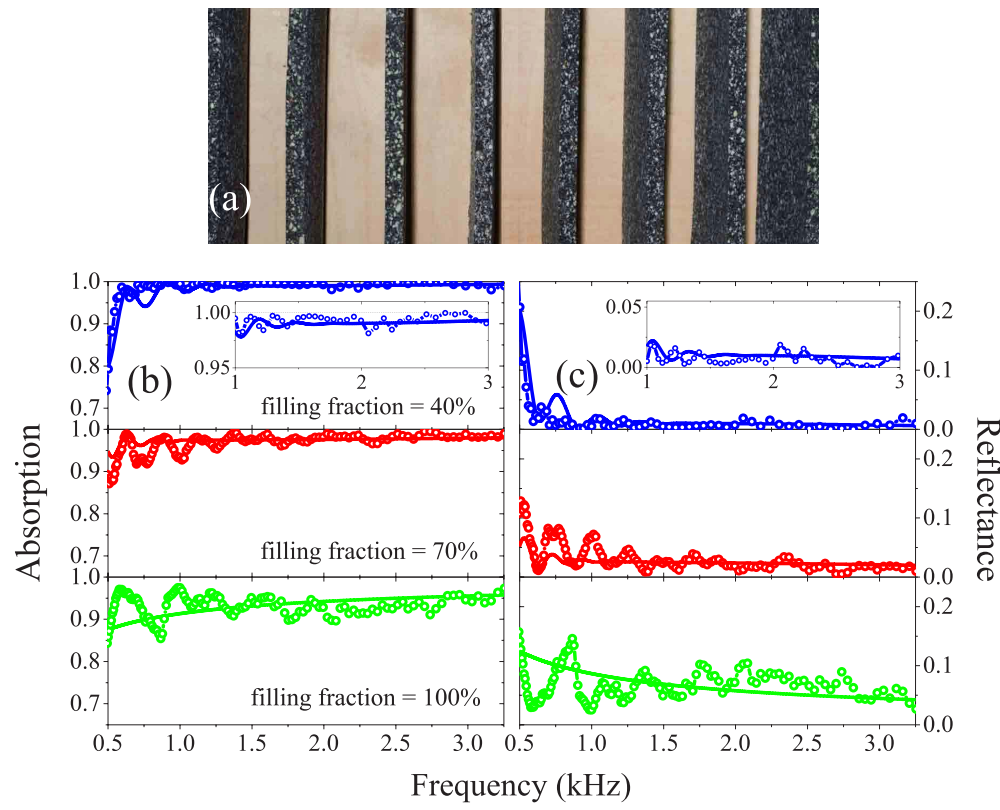

Figure $3 \mid$ Measured and simulated absorption and reflectance demonstrating increased dissipation when lowering the filling fraction. (a), Bottom view on the suspended crystal made out of porous foam lamellas and supported by a rigid backing. The lamellas have the width $\mathrm{W}=4 \mathrm{~cm}$ and length $L=$ $0.5 \mathrm{~m}$. (b,c), Measured (open circles) and simulated (full lines) absorption and reflectance versus frequency for three different filling fractions, $\mathrm{f}=40 \%$, $70 \%$ and $100 \%$. All data are obtained for a normal incident acoustic plane wave.

$\gamma>1$, meaning that dissipation inside the crystal exceeds the intrinsic material losses of the lamellas. From Eq. (3) it follows that reflectance (absorption) will decrease (increase) whenever wave slowing or increased dissipation take place. Since we will not operate within the long wavelength regime, we apply this model in our interpretation of the enhanced absorption because homogenization of the lamella-crystal cannot be applied safely.

We examine the structure depicted in Fig. la where the goal is to explore the tunability for maximal absorption. The basic structure is a 1D sonic crystal consisting of an array of lossy slices supported by a perfectly rigid backing. With translational invariance in the $y$ direction we numerically simulate the complex wave interaction by coupling free-space sound radiation to Bloch states inside the crystal (detailed derivations are found in the Methods section). This leads to the following expression for the reflection coefficient

$$
r_{G}=2 \sum_{j} p_{j}^{G} B_{j} \cos q_{j} L-\delta_{0 G}
$$

where $p_{j}^{G}, B_{j}$ and $q_{j}$ are the $j$ th eigenvector, modal amplitude and outof-plane wave vector of the crystal respectively, from which we derive the overall absorption

$$
A=1-\sum_{G} \operatorname{Re}\left(\frac{k_{z}}{k_{z}^{G}}\right)\left|r_{G}\right|^{2},
$$

where $k_{z}^{G}=\sqrt{k_{0}^{2}-\left(k_{x}+G_{x}\right)^{2}}$. We begin by analysing the spectral absorption $A$ (Eq. (5)) for sound incident along the out-of-plane crystal orientation at normal incidence. In the experimental set-up we utilize a porous material based on homogeneous foam, hence to conduct numerical simulations we have implemented dispersive material dependence for these lossy materials and calculated the acoustical response of the crystal to an incoming sound wave $e^{22}$. In order to lower the filling fraction and by this controlling the overal dissipation, we need to change the lattice constant for a fixed lamella width. By inspecting the bulk properties for the case when $\mathrm{f}=100 \%$ or equivalently $\gamma=1$ we predict overall weak absorption for low frequencies as seen in Fig. 1c. Obviously this is the regime to be challenged since porous materials naturally perform most efficient at higher frequencies. Changing the crystal filling fraction $\mathrm{f}$ by varying the lattice parameter $a$ causes the lattice singularities to shift accordingly as seen in Fig. 1c. These spectral dips resulting in low absorptions arise from diffracted waves becoming grazing and take place when $\left|k_{x}+G_{x}\right|=\omega / c_{0}{ }^{23}$. If the filling fraction is lowered, a growth in the enhancement factor is seen, which is giving rise to increased absorption. This increase in dissipation is simply explained by the fact that sound is trapped more efficiently inside the crystal as compared to the bulk $(\mathrm{f}=100 \%)$ meaning that $\gamma$ must be larger than unity as depicted in Fig. 1b. We obtain enhanced absorption caused by an increased acoustic interaction strength $(\gamma>1)$ that for higher frequencies slightly grows inversely with the filling fraction, see Fig. 1b. On the other hand, one could in as much create systems with complete absorption $\gamma>1$ of sound by designing lamellas made out of an intrinsic lossier material. It is the choice of materials with high absorption rates and the resulting delay time within the dispersive crystal which dictates the performance.

Further to this, we simulate the dependence of the absorption with lamella length $L$. At a given frequency the waves penetrate into the crystal with a characteristic length that should not surpass the crystal length $L>l_{p}$ to avoid strong back-reflection at the rigid support. For this reason, either the wave needs to decay rapidly or travel a sufficient long distance to undergo enough intensity attenuation as plotted in Fig. 2a, showing that absorption increases when the structure becomes lengthy. In Fig. 2a we also observe some fine oscillations in the mapping of which the spectral locations scale with $1 / L$ as known from cavity resonances. More importantly however, although 
we predict high absorptions in the range $0.95-0.999$ in a representative band between 2.0 to $2.5 \mathrm{kHz}$ for $L=0.1 \mathrm{~m}$, as seen in Fig. 2a the bandwidth of complete sound absorption is easily broadened by increasing the lamella length $L$. Unlike membrane type absorbers decorated with rigid platelets where the absorption is resonancebased and overall narrow in width ${ }^{1}$, the present scheme relying on diffraction constitutes an acoustically thick layer which is essential for broadband applications. Next, we investigate the angular sensitivity for the crystal, which up to this point has been simulated for normal incident acoustic plane waves only. For this, it is useful to calculate the absorption versus frequency and parallel momentum $k_{x}$ within the first Brillouin zone. Fig. $2 c$ shows this band diagram containing Bragg-folded sound lines which are responsible for the lattice singularities of low absorption. The entire angular and spectral response resembles the one seen with bandgap materials with no material contrast, but for the present case comprising out-of-plane propagation, it is shown that near to $100 \%$ absorption sustains for the angles shown within the first Brillouin zone (see Fig. 2b for specific frequency cuts). Beyond this zone, absorption remains high up to grazing incidence and the limits are met only whenever irradiation is in phase with the crystal lattice and full reflection takes place.

Experimental verifications. The crystal is constructed out of thin layers of homogeneous foam that is created by compaction and compression of a polyurethane, polyester and polyether mixture. These lamellas have a width of $\mathrm{W}=4 \mathrm{~cm}$ and are arranged into a 1D lattice, which is suspended and backed by a rigid wood support as illustrated in Fig. 3a. We have applied the transfer function method consisting of a loudspeaker and two microphones for the phase and amplitude measurements to detect complex reflections. In the experimental set-up the loudspeaker is placed at a sufficient distance from the microphones and the sample to ensure plane wave generation for all relevant frequencies (see Methods and supplementary material). Spectrally, we evaluate the reflectance $|r(\omega)|^{2}$ and the resultant absorption $A$ over frequency ranges relevant to road and air traffic-noise screening. From the previous study we predicted that lowering the crystal filling fraction will improve the absorption of sound due to the enhancement of the interaction strength. To validate this experimentally we have constructed various samples made out of the same lamellas but varied the size of the unit cell resulting in three different filling fraction as seen in Fig. 3. Bulk material properties are obtained measuring the response of the structure with a filling fraction of $100 \%$ from which relatively strong absorption stems from intrinsic material losses and the slab length $L$. When lowering the filling fraction down to $70 \%$ and $40 \%$, we observe increased performances with mean absorption of 0.97 and 0.99 , respectively, over an extended spectral range spanning from 0.7 to $3 \mathrm{kHz}$. Due to a finite number of unit cells (see Methods) we detect oscillations in the spectrum, overall however, the theory agrees very well with the average absorption evaluated from experiments. The crystal fabricated with a filling fraction of $40 \%$ does not only outperform the other samples measured, see insets of Fig. 3, but further to this we measure a reflectance on the order of $10^{-4}$ in a representative frequency range.

Due to the interaction strength exceeding unity, complete sound absorption extends much further away from normal incidence for various directions $\left(k_{x}=k_{0} \sin \theta\right)$ as rendered in Fig. $2 \mathrm{c}$. We conducted angular-resolved absorption measurements where we vary the angle $\theta$ from the normal to the crystal surface. Caused by diffraction associated by momentum transfer to the lattice (dash-dotted lines), again we predict regions of higher reflections as seen in Fig. 4. This narrow region is however being surpassed by a spectrally broad region of strong absorption spanning from $\theta=0^{\circ}-40^{\circ}$. Both, the finite number of unit cells and the rigid rotatable frame used for the angular
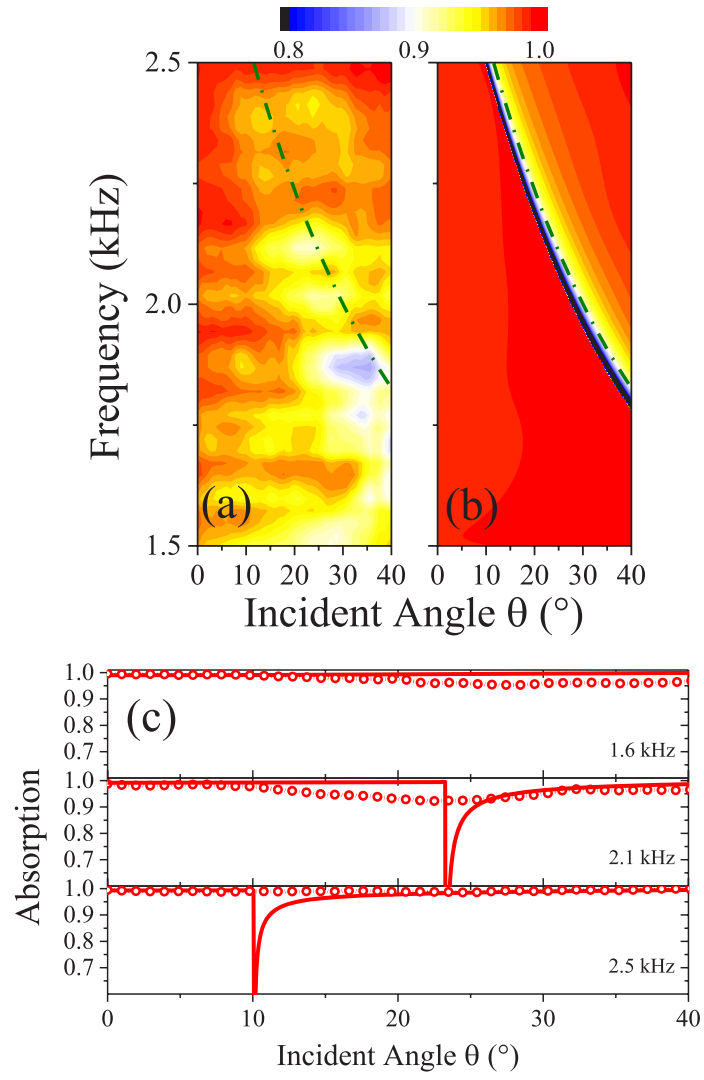

Figure $4 \mid$ Measured and simulated angle-resolved absorption spectrum. (a), Measured (see Methods for details) and (b), simulated absorption as a function of frequency and angle of incidence for the same structure as in the previous measurements, now just with $\mathrm{f}=36 \%$. The dash-dotted line on both contour-maps indicate the condition where $\left|k_{x}+G_{x}\right|=\omega / c_{0}$. (c), Angular dependence of the absorption, representing three measured (open circles) and simulated (full lines) frequency cuts from the contours in (a) and (b).

measurements (see Methods) are causing additional unwanted reflections in the measurements, Fig. 4a. However, upon inspecting both experimentally and numerically the absorption for three different frequencies within the entire resolved spectra (Fig. 4a and $4 \mathrm{~b}$, respectively), we find overall good agreement validating broadband absorption for almost any direction as seen in Fig. 4c.

\section{Discussion}

We have shown a new concept to engineer highly absorptive acoustic materials by means of increasing the sound-material interaction strength which is leading to vanishing reflectance, hence, perfect absorption of sound. We have achieved this goal by fabricating crystals made out of intrinsically lossy lamellas and mounted them onto a rigid backing. For a representative broad range of frequencies we show that absorption can be controlled by the crystal density and tuned up to $99 \%$. The counterintuitive ability to increase absorption by lowering the effective amount of material is providing many interesting opportunities for minimizing noise pollutions by producing efficient acoustic sealing. 


\section{Methods}

Numerical simulation. We have implemented a plane wave expansion technique comprising in-plane Bloch waves and out-of-plane harmonic pressure waves $p=e^{i \omega t} e^{i q z} \sum p^{G} e^{i\left(k_{x}+G\right) x}$ where $q$ is the out-of-plane wave vector, $k_{x}+G$ with

$G=G_{x}=n \frac{2 \pi}{a} \hat{x}$, the in-plane wave vector and $p^{G}$ the eigenvector. After expanding the fields and the complex material parameters $\rho$ and $K$ we end up with the following set of equations:

$$
\sum_{G^{\prime}}\left(\frac{1}{\rho_{G-G^{\prime}}}\left(k_{x}+G\right) \cdot\left(k_{x}+G^{\prime}\right)+q^{2} \frac{1}{\rho_{G-G^{\prime}}}-\omega^{2} \frac{1}{K_{G-G}}\right) p^{G^{\prime}}=0 .
$$

Since external radiation impinges onto the crystal that is backed by a rigid support (see Fig. 1a), we need to treat those two regions separately. We write the free-space wave radiation in the form of an incident and back-scattered wave

$$
p_{I}=e^{i k_{x} x} e^{i k_{z} z}+\sum_{G} r_{G} e^{i\left(k_{x}+G\right) x} e^{-i k_{z}^{G} z},
$$

and the crystal cavity modes as

$$
p_{I I}=2 \sum_{G} \sum_{j} p_{j}^{G} B_{j} \cos q_{j}(z-L) e^{i\left(k_{x}+G\right) x} .
$$

In this cavity, the wave amplitudes are weighted over the $j$ th eigenvector $p_{j}^{G}$ and modal amplitude $B_{j}$ as shown in Eq. (8). When imposing continuity of the fields at the interface, we solve the unknows as for example the reflection coefficient $r_{G}$ and the overall absorption $A$ (Eq. (4) and Eq. (5) respectively).

Experimental set-up. The crystal, which is backed by a rigid wood panel, contains 9 unit cells of lamellas of width $\mathrm{W}=4 \mathrm{~cm}$, length $L=50 \mathrm{~cm}$ and depth $D=100 \mathrm{~cm}$ that are mounted upside down without weight suspensions, see Fig. 3a. For the angleresolved measurements, the crystal was mounted on a rotatable frame with a vertical axis. In order to fit the 9 unit cells inside this frame, the crystal was constructed with a filling fraction of $f=36 \%$.

As rendered in Fig. S1 (supplementary material), the experimental set-up constructed to measure the complex reflection coefficient $r(\omega)$ is based on the transfer function method and applied in the anechoic chamber. The first microphone has been placed at a sufficiently large distance to the source to ensure propagation of plane waves for the entire range of frequencies analysed. Moreover, to avoid finite size effects of the truncated crystal, the second microphone is placed in the nearest proximity to the crystal. For the sound excitation we use a Genelec 8130A Digital Monitoring System placed with a distance of $d=4 \mathrm{~m}$ to the first microphone. Two B\&K 4189 microphones of $1 / 2$ inch are placed at the equatorial plane of the crystal and aligned with the central unit cell. Data from the two microphones are acquired with a CLIO $10 \mathrm{FW}$ (Audiomatica) and then processed in a computer.

1. Mei, J. et al. Dark acoustic metamaterials as super absorbers for low-frequency sound. Nat. Commun. 3, 756 (2012).

2. Leroy, V., Strybulevych, A., Scanlon, M. G. \& Page, J. Transmission of ultrasound through a single layer of bubbles. Eur. Phys. J. E 29, 123 (2009).

3. Leroy, V., Bretagne, A., Fink, M. H. W., Tabeling, P. \& Tourin, A. Design and characterization of bubble phononic crystals. Appl. Phys. Lett. 95, 171904 (2009).

4. Thomas, E. L. Applied physics: Bubbly but quiet. Nature 462, 990 (2009).

5. Romero-García, V., Sánchez-Pérez, J. V. \& Garcia-Raffi, L. M. Tunable wideband bandstop acoustic filter based on two-dimensional multiphysical phenomena periodic systems. J. Appl. Phys. 110, 014904 (2011).

6. Garcia-Chocano, V. M., Cabrera, S. \& Sanchez-Dehesa, J. Broadband sound absorption by lattices of microperforated cylindrical shells. Appl. Phys. Lett. 101, 184101 (2012)

7. Kushwaha, M. S., Halevi, P., Dobrzynski, L. \& Djafari-Rouhani, B. Acoustic band structure of periodic elastic composites. Phys. Rev. Lett. 71, 2022 (1993).

8. Vasseur, J. O. et al. Experimental and Theoretical Evidence for the Existence of Absolute Acoustic Band Gaps in Two-Dimensional Solid Phononic Crystals. Phys. Rev. Lett. 86, 3012 (2001).
9. Liu, Z. et al. Locally Resonant Sonic Materials. Science 289, 1734 (2000).

10. Christensen, J., Martin-Moreno, L. \& Garcia-Vidal, F. J. All-angle blockage of sound by an acoustic double-fishnet metamaterial. Appl. Phys. Lett. 97, 134106 (2010).

11. Botten, L. C., Craig, M. S., McPhedran, R. C., Adams, J. L. \& Andrewartha, J. R. The finitely conducting lamellar diffraction grating. Optica Acta 28, 1087 (1981)

12. McPhedran, R. C., Botten, L. C., Craif, M. S., Neviere, M. \& Maystre, D. Lossy lamellar gratings in the quasistatic limit. Optica Acta 29, 289 (1982).

13. Kravets, V. G., Schedin, F. \& Grigorenko, A. N. Plasmonic blackbody: Almost complete absorption of light in nanostructured metallic coatings. Phys. Rev. B 78, 205405 (2008).

14. Sondergaard, T. et al. Plasmonic black gold by adiabatic nanofocusing and absorption of light in ultra-sharp convex grooves. Nat. Commun. 3, 969 (2012)

15. Clapham, P. B. \& Hurtley, M. C. Reduction of Lens Reflexion by the Moth Eye Principle. Nature Vol. 244, 281 (1973).

16. Garcia-Vidal, F. J., Pitarke, J. M. \& Pendry, J. B. Effective Medium Theory of the Optical Properties of Aligned Carbon Nanotubes. Phys. Rev. Lett. 78, 4289 (1997).

17. Yang, Z., Ci, L., Bur, J. A., Lin, S. \& Ajayan, P. M. Experimental Observation of an Extremely Dark Material Made By a Low-Density Nanotube Array. Nano Lett. 8 446 (2008).

18. Garcia-Vidal, F. J. Metamaterials: Towards the dark side. Nat. Photonics 2, 215 (2008).

19. Mizunoa, K. et al. A black body absorber from vertically aligned single-walled carbon nanotubes. Proc. Natl. Acad. Sci. USA 106, 6044 (2009).

20. Lidorkis, E. \& Ferrari, A. C. Photonics with Multiwall Carbon Nanotube Arrays. ACS Nano 3, 1238 (2009).

21. Beenakker, C. W. J. \& Brouwer, P. W. Distribution of the reflection eigenvalues of a weakly absorbing chaotic cavity. Physica E 9, 463 (2001).

22. Lafarge, D., Lemarinier, P., Allard, J. F. \& Tarnow, V. Dynamic compressibility of air in porous structures at audible frequencies. J. Acoust. Soc. Am. 102, 1995 (1997), With the macroscopic parameters: $\phi=0.94, \alpha_{\infty}=1, \sigma=20000 \mathrm{Nm}^{-4} \mathrm{~s}$ and $\Lambda=\Lambda^{\prime}=0.41 \mu \mathrm{m}$.

23. García de Abajo, F. J. Colloquium: Light scattering by particle and hole arrays. Rev Mod. Phys. 79, 1267-1290 (2007)

\section{Acknowledgments}

The work was supported by the Spanish Ministry of Science and Innovation and European Union FEDER through project FIS2011-29734-C02-01. J.C. gratefully acknowledges financial support from the Danish Council for Independent Research and a Sapere Aude grant (12-134776). V.R.G. gratefully acknowledges financial support from the "Contratos Post-Doctorales Campus Excelencia Internacional" UPV CEI-01-11.

\section{Author contributions}

J.C., F.J.G.A., N.A.M. and M.W. developed the numerical tool and undertook the analytical analysis, V.R.-G., R.P., A.C. and V.J.S.-M. designed and conducted all experimental measurements. J.C. conceived the idea and wrote the manuscript and all authors participated in the revision.

\section{Additional information}

Supplementary information accompanies this paper at http://www.nature.com/ scientificreports

Competing financial interests: The authors declare no competing financial interests

How to cite this article: Christensen, J. et al. Extraordinary absorption of sound in porous lamella-crystals. Sci. Rep. 4, 4674; DOI:10.1038/srep04674 (2014).

This work is licensed under a Creative Commons Attribution-NonCommercialNoDerivs 3.0 Unported License. The images in this article are included in the article's Creative Commons license, unless indicated otherwise in the image credit; if the image is not included under the Creative Commons license, users will need to obtain permission from the license holder in order to reproduce the image. To view a copy of this license, visit http://creativecommons.org/licenses/by-nc-nd/3.0/ 



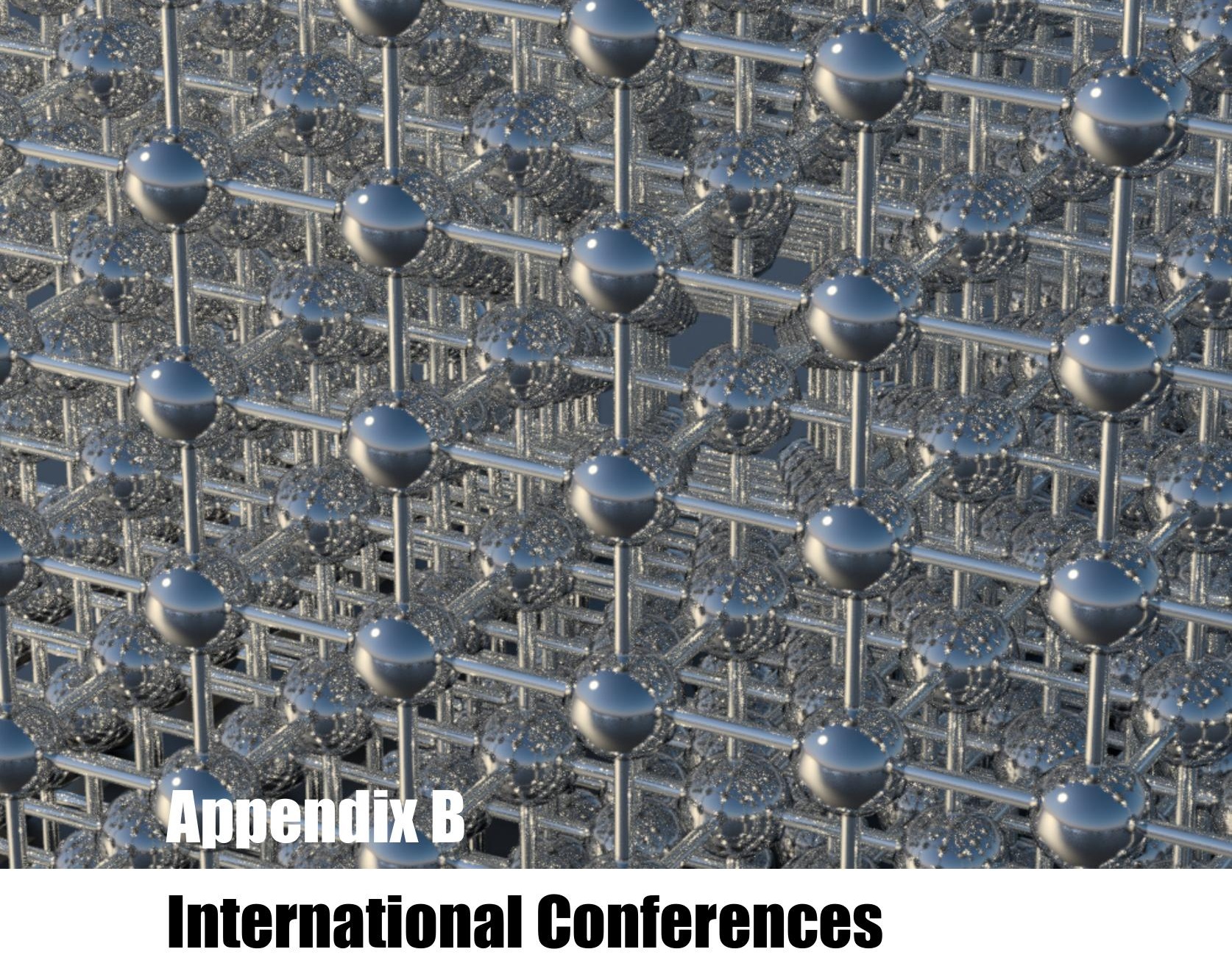

1. Salmerón-Contreras, L. Cebrecos, A. Jiménez, N. García-Raffi, L. M. Picó Vila, R. Redondo, J. Romero-García, V. Sánchez-Morcillo, V. J. Staliunas, K.

Diffusion of Sound Waves by Sonic Crystals.

3rd International Conference on Phononic Crystals/Metamaterials, Phonon Transport and and Phonon Coupling (PHONONICS 2015).

Paris, France. 2015.

2. Cebrecos, A. Krattiger, D. Maute, K. Sánchez-Morcillo, V. J. Park, K. C. Oh, I. K.

Fluidic Metamaterial: An Elastic Medium with a Time-changing Band 
Structure.

3rd International Conference on Phononic Crystals/Metamaterials, Phonon Transport and and Phonon Coupling (PHONONICS 2015).

Paris, France. 2015.

3. Cebrecos, A. García-Raffi, L. M. Picó Vila, R. Romero-García, V. SalmerónContreras, L. Sánchez-Morcillo, V. J. Staliunas, K.

Sound Wave Enhancement in Chirped Structures.

3rd International Conference on Phononic Crystals/Metamaterials, Phonon Transport and and Phonon Coupling (PHONONICS 2015).

Paris, France. 2015.

4. Mehrem, A. Hamham, E. M. Jiménez, N. Cebrecos, A. Picó Vila, R. Sánchez-Morcillo, V. J. García-Raffi, L. M.

Nonlinear Acoustic Waves Periodic Media.

7th Forum Acusticum 2014.

Cracow, Poland. 2014.

5. Romero-García, V. García-Raffi, L. M. Cebrecos, A. Picó Vila, R. SánchezMorcillo, V. J.

Acoustic fractal conical lens based on sonic crystals.

2nd International Conference on Phononic Crystals/Metamaterials, Phonon Transport and Optomechanics (PHONONICS 2013).

Sharm el-Sheikh, Egypt. 2013.

6. Picó Vila, R. Romero-García, V. Cebrecos, A. Sánchez-Morcillo, V. J. Maygite, L. Herrero, R. Botey, M. Staliunas, K.

AntiBandgaps in Acoustic Periodic Loss Media.

2nd International Conference on Phononic Crystals/Metamaterials, Phonon Transport and Optomechanics (PHONONICS 2013).

Sharm el-Sheikh, Egypt. 2013. 
7. Cebrecos, A. Romero-García, V. Picó Vila, R. Sánchez-Morcillo, V. J. García-Raffi, L. M. Sánchez-Pérez, J. V.

Axisymmetric toroidal system for beam focalization.

2nd International Conference on Phononic Crystals/Metamaterials, Phonon Transport and Optomechanics (PHONONICS 2013).

Sharm el-Sheikh, Egypt. 2013.

8. Cebrecos, A. Romero-García, V. Picó Vila, R. Sánchez-Morcillo, V. J. Adkins, N.

Collimation of ultrasound beams behind 3D sonic crystal.

2nd International Conference on Phononic Crystals/Metamaterials, Phonon Transport and Optomechanics (PHONONICS 2013).

Sharm el-Sheikh, Egypt. 2013.

9. Romero-García, V. Cebrecos, A. Picó Vila, R. Sánchez-Morcillo, V. J. Staliunas, K

Enhancement of Sound in Chirped Sonic Crystals.

2nd International Conference on Phononic Crystals/Metamaterials, Phonon Transport and Optomechanics (PHONONICS 2013).

Sharm el-Sheikh, Egypt. 2013.

10. Romero-García, V. Cebrecos, A. Picó Vila, R. Sánchez-Morcillo, V. J. Botey, M. Herrero, R. Cheng, Y. C. Staliunas, K.

Unlocked Evanescent Waves in Sonic Crystals.

2nd International Conference on Phononic Crystals/Metamaterials, Phonon Transport and Optomechanics (PHONONICS 2013).

Sharm el-Sheikh, Egypt. 2013.

11. Romero-García, V. Picó Vila, R. Cebrecos, A. García-Raffi, L. M. SánchezPérez, J. V. Sánchez-Morcillo, V. J. 
Focusing properties of axisymmetric acoustic metamaterials made of toroidal scatterers.

ASME 2012 International Mechanical Engineering Congress \& Exposition .

Houston, Texas. USA. 2012.

12. Romero-García, V. Cebrecos, A. Picó Vila, R. Sánchez-Morcillo, V. J. Botey, M. Herrero, R. Cheng, Y. C.

Focusing of evanescent waves by modulated sonic structures.

9th International Conference on Theoretical and Experimental Topics in the Field of Electrical, Transport, and Optical Properties of Inhomogeneoues Media (ETOPIM 2012).

Marselle, France. 2012.

13. Cebrecos, A. Picó Vila, R. Romero-García, V. Redondo, J. SánchezMorcillo, V. J. Staliunas, K.

Reflection of acoustic beams in sonic crystals.

9th International Conference on Theoretical and Experimental Topics in the Field of Electrical, Transport, and Optical Properties of Inhomogeneoues Media (ETOPIM 2012).

Marselle, France. 2012.

14. Romero-García, V. Cebrecos, A. Picó Vila, R. Sánchez-Morcillo, V. J. Staliunas, K.

Spatial filtering and complex isofrequency contours in phononic crystals. 3rd International Conference on Metamaterials, Photonic Crystals and Plasmonics (META 2012).

Paris, France 2012.

15. Cebrecos, A. Romero-García, V. Picó Vila, R. Sánchez-Morcillo, V. J. García-Raffi, L. M. Sánchez-Pérez, J. V. Staliunas, K. 
Influence of periodicity on the reflection properties of finite periodic media.

162nd Meeting of the Acoustical Society of America.

San Diego, USA. 2011. 



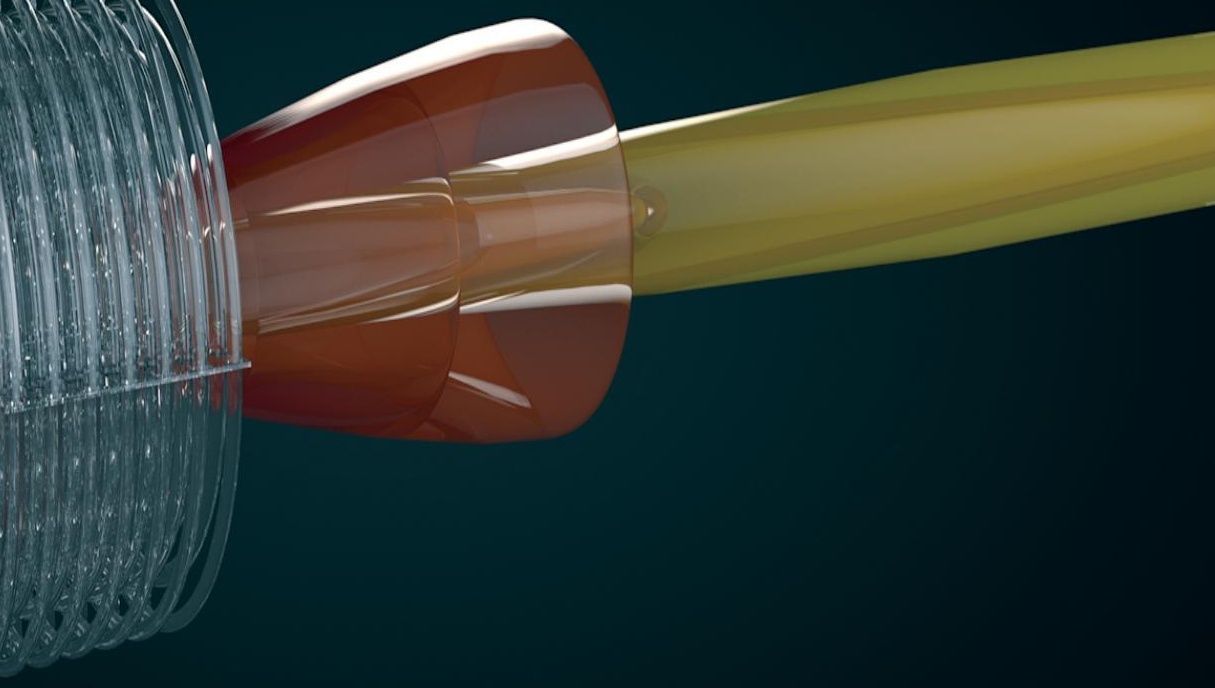

\section{Appendix $\theta$}

\section{Awards}

\section{European Physical Society}

EPS Second Place Best Student Oral Presentation Award.

Phononics 2015. 3nd International Conference on Phononic Crystals/Metamaterials, Phonon Transport \& Phonon Coupling.

Paris, France. 2015.

\section{Office of Naval Research Global}

ONR Global Second Place Best Student Poster Presentation Award.

Phononics 2013. 2nd International Conference on Phononic Crystals/Metamaterials, Phonon Transport \& Optomechanics.

Sharm-El-Sheikh, Egypt. 2013. 
